Copyright

by

Gabriel Noam Price

2009 
The Dissertation Committee for Gabriel Noam Price certifies that this is the approved version of the following dissertation:

\section{Single-Photon Atomic Cooling}

Committee:

Mark G. Raizen, Supervisor

Herbert L. Berk

Arno Bohm

John W. Keto

Pawan Kumar 


\title{
Single-Photon Atomic Cooling
}

\author{
by
}

Gabriel Noam Price, B.A.

\author{
DISSERTATION \\ Presented to the Faculty of the Graduate School of \\ The University of Texas at Austin \\ in Partial Fulfillment \\ of the Requirements \\ for the Degree of \\ DOCTOR OF PHILOSOPHY
}

THE UNIVERSITY OF TEXAS AT AUSTIN

August 2009 
Dedicated to my parents, Anne and Neal Price, and to Elizabeth, my lovely bride to be, whose love and support keeps me going each day. 


\section{Acknowledgments}

First, I would like to thank my supervisor, Dr. Mark G. Raizen. Never before have I met an individual with so many wonderful and inspiring ideas. His ability to see directly to the heart of physical problems and identify their fundamental issues is astounding. Whenever the project ran into what seemed to be serious trouble, Mark would give us a multitude of ideas to circumvent it. He has served as an endless source of ideas, inspiration, and motivation (when needed). I could not have asked for a better advisor and am thankful that he allowed me to join the group in the summer of 2003.

This brings me to the wonderful group of people that I have had the pleasure of working alongside throughout these years. A particular thanks and acknowledgment goes to Travis Bannerman. He joined the group a little before my qualifier and has worked on this project with me for almost its entire duration. He is an extremely smart individual with keen physical instincts. He worked very hard, contributed many ideas and helped to make the project a success. I have no doubt that he will bring the experiment to the next level successfully and will continue to do great work beyond this lab, in whatever field he chooses. Next, I would like to thank Kirsten Veiring. She originally joined this lab as a Würzburg master student and did excellent work determining "magic wavelengths" for Sodium and Rubidium. Apparently she enjoyed 
her time, and the lab's students, so much that she decided to return to do her doctoral work. When she did so, she joined me in the Rubidium experiment and helped to conclude much of the work discussed in this dissertation. She has a real eye for detail and a tremendously bright future.

I would also like to thank other students in the lab who have made my time here so enjoyable. Hrishi has been in the lab the entire time I have. We both became the senior students on our respective projects around the same time. He has always been extremely friendly and helpful. On several occasions when I did not have a needed piece of equipment and he did, he gladly loaned it to me so that I could precede. Tongcang Li has tremendous physical intuition and is always up for an entertaining discussion on one topic or another. Whenever I was stressed, he always seemed to know how to help me put thing into perspective. Adam Libson is a great experimentalist that I have gotten to know well over the years through countless lunches together. His love of physics will take him far. David Medellin is fantastic with equations and has helped many people in the lab, including myself, by clarifying derivations. Isaac Chavez began as an undergraduate in our lab and made the transition into graduate student. He is an extremely hard worker from whom I expect great things. Tom Mazur is the newest member of our group and, in addition to being a really nice person, has started his graduate studies strong.

Of course, when I first joined the group its composition was quite different, and I would like to thank those students who I encountered at that time. Todd Meyrath, Chih-Sung Chuu and Florian Schreck comprised the 
Rubidium group when I first joined. Together, along with Jay Hanssen, they constructed a Rubidium BEC apparatus which would later be converted into the experimental apparatus used by me during the work outlined in this dissertation. Todd designed and constructed most of the electronic control systems and I had the pleasure of working closely with him my first year. He introduced me to the electronics controlling our experiment and was an excellent and patient teacher. Florian was the lab postdoc at that time and he wrote the excellent software which we still use to control the experiment. He served as a major force pushing the experiment along at that time. I worked along side Chih-Sung during the time period he performed what would become his doctoral work. I learned a lot by watching his careful experimental technique and methodical parameter-space searches. Kevin Henderson led the sodium experiment at that time and his meticulous attention to detail and order as well as his tremendous work ethic rubbed off on me a little. He always made the lab a more pleasant place with his hilarious stories.

Finally I would like to thank individuals outside of the lab who played a very helpful role. Allen Schroeder heads a first rate machine shop full of extremely talented individuals. The hard work they put into constructing many of the apparatus used during the experiment is greatly appreciated. Jack Clifford runs the student shop. He is always friendly and ready to go out of his way to help people accomplish their machining goals. I would like to thank him for further honing my precision machining skills. The Center for Nonlinear Dynamics administrative staff head, Olga Vera, made sure that my 
purchase orders when through fast, that my tuition got paid on time, and that I got my stipend.

Gabriel N. Price Austin, TX

August 2009 


\title{
Single-Photon Atomic Cooling
}

\author{
Publication No. \\ Gabriel Noam Price, Ph.D. \\ The University of Texas at Austin, 2009
}

Supervisor: Mark G. Raizen

This dissertation details the development and experimental implementation of single-photon atomic cooling. In this scheme atoms are transferred from a large-volume magnetic trap into a small-volume optical trap via a single spontaneous Raman transition that is driven near each atom's classical turning point. This arrangement removes nearly all of an atomic ensemble's kinetic energy in one dimension. This method does not rely on a transfer of momentum from photon to atom to cool. Rather, single-photon atomic cooling achieves a reduction in temperature and an increase in the phase-space density of an atomic ensemble by the direct reduction of the system's entropy. Presented here is the application of this technique to a sample of magnetically trapped ${ }^{87} \mathrm{Rb}$. Transfer efficiencies between traps of up to $2.2 \%$ are demonstrated. It is shown that transfer efficiency can be traded for increased phase-space compression. By doing so, the phase-space density of a magnetically trapped ensemble is increased by a factor of 350 by the single-photon atomic cooling process. 


\section{Table of Contents}

Acknowledgments $\quad$ v

$\begin{array}{ll}\text { Abstract } & \text { ix }\end{array}$

List of Tables $\quad$ xiii

List of Figures xiv

Chapter 1. Introduction 1

1.1 A Brief History of Laser Cooling . . . . . . . . . . . . . . . . 1

1.2 Laser Cooling as an Enabling Tool . . . . . . . . . . . . . . . . 4

1.3 Limitations of Laser Cooling . . . . . . . . . . . . . . 5

1.4 Introduction to Single-Photon Cooling . . . . . . . . . . . 8

1.5 Single-Photon Cooling as a Maxwell's Demon . . . . . . . . . . 16

1.6 A Note on Units . . . . . . . . . . . . . . . . . . . 19

$\begin{array}{lll}\text { Chapter 2. Operational Concepts } & 24\end{array}$

2.1 Rubidium ........................ . . 25

2.2 Fine and Hyperfine Structure . . . . . . . . . . . . . . 26

2.2.1 Fine Structure . . . . . . . . . . . . . . . . . 26

2.2.2 Hyperfine Structure . . . . . . . . . . . . . . . . . . 29

2.3 The Zeeman Effect . . . . . . . . . . . . . . . . . . . 34

2.4 Magnetic Trapping . . . . . . . . . . . . . . . . . . . 40

2.5 Interaction of Light with Atoms . . . . . . . . . . . . . . 45

2.5.1 The Optical Dipole Force . . . . . . . . . . . . . 45

2.5.2 Scattering Forces . . . . . . . . . . . . . . . . . . . . . . . . . . . . . . . . . .

2.5.2.1 Optical Bloch Equations . . . . . . . . . . 50

2.5.2.2 Optical Molasses . . . . . . . . . . . . . . . . . 55

2.5.2.3 Sisyphus Cooling . . . . . . . . . . . . 59 
2.5.2.4 Magneto-Optical Trap . . . . . . . . . . . . 64

2.6 Branching Ratios . . . . . . . . . . . . . . . . . . 68

2.7 Laser Frequency Control . . . . . . . . . . . . . . . . . . . . 72

2.7.1 Doppler Broadening . . . . . . . . . . . . . . . . 72

2.7.2 Saturation Absorption Spectroscopy . . . . . . . . 75

2.7.3 Laser Frequency Lock . . . . . . . . . . . . . . 78

2.8 Imaging . . . . . . . . . . . . . . . . . . . . . . . . . . . . . . . 79

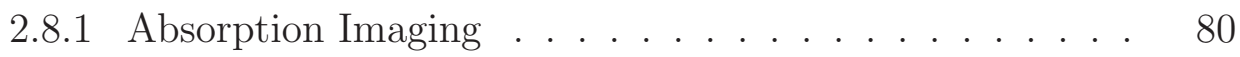

2.8.2 Fluorescence Imaging . . . . . . . . . . . . . . 83

Chapter 3. $\quad$ Experimental Apparatus $\quad 85$

3.1 Vacuum Chamber . . . . . . . . . . . . . . . 85

3.1 Upper Chamber . . . . . . . . . . . . . . 86

3.1.2 Middle Chamber . . . . . . . . . . . . . . . 90

3.1.3 Lower Chamber . . . . . . . . . . . . . . . . . . . . . 93

3.2 Lasers . . . . . . . . . . . . . . . . . . . . . . . . . . 95

3.2.1 Near-Resonance Lasers . . . . . . . . . . . . . . . 96

3.2.1.1 MOT Master Laser . . . . . . . . . . . . . 98

3.2.1.2 Slave Lasers . . . . . . . . . . . . . . . . . 105

3.2.1.3 Upper MOT Horizontal Slave Laser . . . . . 108

3.2.1.4 Upper MOT Diagonal Slave Laser .... . 110

3.2.1.5 Lower MOT Slave Laser . . . . . . . . . . . . . 112

3.2.1.6 Repump Master Laser . . . . . . . . . . . . . 112

3.2.1.7 Depopulation Beam . . . . . . . . . 116

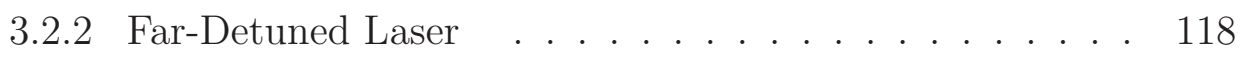

3.3 Magnetic Trap . . . . . . . . . . . . . . . . . . . . . . . . . . 124

3.4 Imaging Systems . . . . . . . . . . . . . . . . . . . . . . 127

3.4 .1 Vertical Imaging . . . . . . . . . . . . . . . 127

3.4.2 Horizontal Imaging . . . . . . . . . . . . . . . . . . . 129 
Chapter 4. Single-Photon Atomic Cooling in ${ }^{87} \mathrm{Rb}$

4.1 Overview of Single-Photon Cooling ${ }^{87} \mathrm{Rb}$. . . . . . . . . . . . 131

4.2 Crossed Dipole Beam Configuration . . . . . . . . . . . . . . . 138

4.3 "Optical Box" Configuration . . . . . . . . . . . . . . 145

4.4 "Optical Trough" Configuration . . . . . . . . . . . . . 156

4.5 Future Directions . . . . . . . . . . . . . . . . . . . . 179

$\begin{array}{lc}\text { Bibliography } & 183\end{array}$

$\begin{array}{ll}\text { Vita } & 196\end{array}$ 


\section{List of Tables}

$2.1{ }^{87}$ Rb Physical Properties . . . . . . . . . . . . . . 26

$2.2{ }^{87} \mathrm{Rb} D_{2}$ Transition Hyperfine Structure Constants . . . . . . . 32

$2.3{ }^{87} \mathrm{Rb} D_{2}$ Transition "g-factors" . . . . . . . . . . . . . . 38

3.1 Verdi V10 system specifications. . . . . . . . . . . . . . 119 


\section{List of Figures}

1.1 A Two-Level Atom . . . . . . . . . . . . . . . . . . . 6

1.2 A Three-Level Atom . . . . . . . . . . . . . . . . 7

1.3 One-Way-Wall . . . . . . . . . . . . . . . . . . . . . . 10

1.4 Simple One-Way-Wall Cooling Scheme . . . . . . . . . . . . . 11

1.5 One-Way-Wall Cooling Scheme in an External Potential . . . 14

1.6 Cooling an Atomic Ensemble . . . . . . . . . . . . . . . . . 15

1.7 Reconstruction of an Initial Energy Distribution . . . . . . . . 18

1.8 Trap Depth . . . . . . . . . . . . . . . . . . . . . . . . . . 21

$2.1{ }^{87} \mathrm{Rb} D_{2}$ Transition Hyperfine Structure . . . . . . . . . . . . . 33

2.2 Vector Model of the Hyperfine Interation . . . . . . . . . . . . 35

2.3 Zeeman Spitting of the $F=1$ Hyperfine Manifold . . . . . . . 39

2.4 Magnetic Field from a Circular Loop . . . . . . . . . . . . . . 42

2.5 Magnitude of the Magnetic Field Produced by the Anti-Helmholtz Configuration . . . . . . . . . . . . . . . 43

2.6 Ensemble of Two-Level Atoms Interacting with a Monochromatic Field . . . . . . . . . . . . . . . . 50

2.7 Geometry of Optical Molasses . . . . . . . . . . . . . 56

2.8 Optical Molasses in 1-D . . . . . . . . . . . . . . . . 57

2.9 Sisyphus Cooling Effect . . . . . . . . . . . . . . . . . . . 61

2.10 Magneto-Optical Trap Geometry . . . . . . . . . . . . . 65

2.11 1-D MOT . . . . . . . . . . . . . . 66

2.12 Decay Mode Branching Ratios from the $\left|F^{\prime}=1, m_{F}=1\right\rangle$ State 71

2.13 The Doppler Effect . . . . . . . . . . . . . . . . . . . . 74

2.14 Typical Saturation Absorption Spectroscopy Layout . . . . . . 75

2.15 Saturation Absorption Spectroscopy: Two-Level Atom . . . . 76

2.16 Cross-over Resonance . . . . . . . . . . . . . . . . . . . 77

2.17 Laser Frequency Locking Layout . . . . . . . . . . . . . 78 
3.1 Vacuum Chamber . . . . . . . . . . . . . . . . . 87

3.2 Upper Vacuum Chamber . . . . . . . . . . . . . . . . . . 88

3.3 Middle Vacuum Chamber . . . . . . . . . . . . . . . . . . . . 92

3.4 Lower Glass Cell . . . . . . . . . . . . . . . . . . . . . . . . 93

3.5 Helicoflex Seal . . . . . . . . . . . . . . . . . . . . . . 95

3.6 Near-Resonance Laser Frequencies . . . . . . . . . . . . . . . . 97

3.7 MOT Master Laser . . . . . . . . . . . . . . . . . . . . . . . . 99

3.8 MOT Master Saturation Absorption Spectroscopy Layout . . . 101

3.9 MOT master saturation absorption signal . . . . . . . . . . . . 103

3.10 MOT Master Beam Distribution . . . . . . . . . . . . . . . . . 104

3.11 Slave Laser . . . . . . . . . . . . . . . . . . . . . . . 106

3.12 Injection of Slave Lasers . . . . . . . . . . . . . . . . 107

3.13 Upper MOT Horizontal Slave Laser Beam Distribution . . . . 109

3.14 Upper MOT Diagonal Slave Laser Beam Distribution . . . . . 111

3.15 Lower MOT Slave Laser Beam Distribution . . . . . . . . . . 112

3.16 Repump Master Saturation Absorption Spectroscopy Layout . 114

3.17 Repump Master Saturation Absorption Signal . . . . . . . . . 115

3.18 Repump Master Beam Distribution . . . . . . . . . . . . . 116

3.19 Depopulation Beam . . . . . . . . . . . . . . . 117

3.20 The Optical Trough . . . . . . . . . . . . . . . . . . . . . 120

3.21 Verdi Beam Distribution . . . . . . . . . . . . . . . . 123

3.22 Picture of Magnetic Trap . . . . . . . . . . . . . . . . . . . . 124

3.23 Picture of Single Quadrupole Coil . . . . . . . . . . . . . . . . 125

3.24 Schematic of Magnetic Trap . . . . . . . . . . . . . . . . 126

3.25 Vertical Probe Beam Path . . . . . . . . . . . . . . . . 128

3.26 Horizontal Probe Beam Path . . . . . . . . . . . . . . . . 129

4.1 Overview of the Single-Photon Cooling Process . . . . . . . . 135

4.2 Depopulation Sheet . . . . . . . . . . . . . . . 137

4.3 Crossed Beam Dipole Trap Geometry . . . . . . . . . . . . . . 139

4.4 Effective Combined Potential in Cross Beam Configuration for the $\left|F=2, m_{F}=2\right\rangle$ state . . . . . . . . . . . . . 140

4.5 Comparison of Effective Potentials . . . . . . . . . . . 141 
4.6 Transition Location . . . . . . . . . . . . . . . . . . . . . 142

4.7 Fluorescence Images with and without Depopulation Beam . . 143

4.8 Temperature of Atoms Cooled in the Cross Beam Configuration 144

4.9 Optical Box Trap . . . . . . . . . . . . . . . . . . . 146

4.10 Calculated Potential due to the Optical Box Trap . . . . . . . 147

4.11 Total Potential in the $\left|F=2, m_{F}=2\right\rangle$ and $\left|F=1, m_{F}=0\right\rangle$ States. . . . . . . . . . . . . . . . 149

4.12 Number of Atoms loaded into Optical Box vs. Depopulation Beam Power . . . . . . . . . . . . . . . . . . 151

4.13 Incremental Atom Capture at a Fixed Translation Velocity . . 153

4.14 Captured Atom Number as a Function of Loading Time . . . 154

4.15 Atoms in Magnetic Trap vs. Gradient . . . . . . . . . . . . . 158

4.16 Geometry of the "Optical Trough" . . . . . . . . . . . . . . . 159

4.17 "Optical Trough" Potential in the $\hat{y}$ - $\hat{z}$ Plane . . . . . . . . . . 160

4.18 "Optical Trough" Potential Side View . . . . . . . . . . . . . . 161

4.19 "Optical Trough" Potential Along $\hat{z}$ and $\hat{x}$. . . . . . . . . . . . 162

4.20 "Optical Trough" and Depopulation Beam Geometry . . . . . 163

4.21 Atom Transfer vs. Depopulation Beam Intensity . . . . . . . . 164

4.22 Good/Bad Scattering Rates vs. Depopulation Beam Detuning 166

4.23 Total Potential for Atoms in the $\left|F=2, m_{F}=2\right\rangle$ and $\mid F=$ $\left.1, m_{F}=0\right\rangle$ State . . . . . . . . . . . 167

4.24 Absorption Image of Atoms in the Optical Trough . . . . . . . 170

4.25 Transfer Number and Vertical Temperature vs. Depopulation Beam Height . . . . . . . . . . . . . . . . . . . . . . 171

4.26 Magnetic Trap Radius vs. Temperature . . . . . . . . . . . . . 175

4.27 Measured Single-Photon Cooling Transfer Efficiencies . . . . . 176

4.28 Magnetically Trapped Atoms Remaining vs. Percentage of Current Ramp down Completed . . . . . . . . . . . . . . . . . . 177

4.29 Atom Accumulation as Quadrupole Current is Ramped Down 178

4.30 Hydrogen Energy Structure Relevant to Single-Photon Atomic Cooling . . . . . . . . . . . . . . . . . . . . 181 


\section{Chapter 1}

\section{Introduction}

This chapter gives a brief introduction to the motivation and experimental methods developed and demonstrated in this dissertation. It begins with a brief discussion of the history of laser cooling. Next, the role laser cooling has played in advancing scientific knowledge is briefly presented. Finally, the method of single-photon atomic cooling, the topic of this dissertation, is presented in general terms and its advantages over established laser cooling techniques as well as its limitations are explored.

\subsection{A Brief History of Laser Cooling}

Laser cooling of atomic vapors has a long and interesting history, however I will only attempt to highlight some key advancements in the field to place the development of single-photon cooling in the proper context. The first experiment in this field was carried out in 1933 by Frisch when he observed the deflection of an atomic beam by resonant light emitted from a sodium lamp [1]. In this experiment the deflection of the atomic beam was due to the scattering of a single resonant photon per atom on average. The important observation was that when a resonant photon is absorbed by an atom, in ad- 
dition to driving an internal transition, it also imparts a momentum kick. The magnitude of momentum transfered from photon to atom is given by the de Broglie relation

$$
|p|=\hbar k
$$

where $k=w / c$ is the magnitude of the wavevector of the resonant light. This landmark experiment demonstrated the possibility of using light to control atomic external degrees of freedom, namely position and momentum. Progress in this field effectively stopped after this observation and further advancement would have to await the invention of the laser.

Interest was revitalized 40 years later with a series of proposals to cool and trap both ions and neutral atoms using the Doppler effect $[2,3]$. The proposed idea is quite ingenious and is worth quickly revisiting here. It involves directing a laser beam tuned slightly below the resonance frequency (red detuned) at an atomic ensemble. The effect of such an arrangement can be deduced by considering what happens to an atom in the ensemble in the following two cases: when the atom travels toward the laser beam and when the atom travels away from it. In the first case, the atom will be brought closer into resonance by the Doppler effect and therefore its scattering rate will increase. In the latter case the motion of the atom will cause it to be further out of resonance with the laser beam, reducing the scattering rate. Note that the absorption of each photon is directional, while the direction of the subsequent spontaneous emission is random. After a large number of scattering events the momentum kicks due to spontaneous emission will average to zero, while 
the momentum kicks due to absorption will sum. The result is that atoms traveling towards the red detuned beam will be slowed by momentum transfer from scattered photons. This arrangement does more than simply slow the center-of-mass motion of an ensemble, it also cools it because the red detuned beam affects atoms in a velocity dependent way. Quickly moving atoms are Doppler shifted to the blue more than slowly moving atoms (assuming both are traveling toward the laser source), causing them to scatter at a greater rate which in turn decelerates them more quickly than more slowly moving atoms. This compresses the velocity spread of the ensemble, thereby cooling it.

Experiments applying this cooling scheme to electromagnetically trapped ions were successfully performed in $1978[4,5]$. Then in 1985 the first experiments demonstrating slowing of neutral atomic beams were published $[6,7]$. That same year, trapping in velocity space with counter propagating red detuned beams, an arrangement known as optical molasses, was demonstrated at Bell Labs by a group led by S. Chu [8]. The spatial confinement of sodium atoms using a magnetic field was also demonstrated in Gaithersburg by the group of W. Phillips [9] in that very productive year of 1985. Two years later, the combination of ideas utilized in these two experiments were used to both confine and cool atoms in a technique known as magneto-optical trapping $(\mathrm{MOT})[10]$.

Shortly after this achievement, groups began to observe and report a troubling, or perhaps welcome, disagreement between experiment and theory. In particular, the group of W. Phillips reported temperatures of atomic sam- 
pled cooled with optical molasses to be much colder than the lower bound set by theory [11] (See Sec. 1.6 for a discussion of the use of "temperature" in this context). This conflict was a result of the assumption made in theoretical descriptions of the process which modeled the atom as a purely two-level system, devoid of magnetic substructure. An improved theory, including magnetic substructure, was developed by C. Cohen-Tannoudji and J. Dalibard in 1989 [12] and it resolved the discrepancy between theory and experiment and placed a new and much colder lower bound temperature, the recoil temperature $T_{r}$, on optical molasses. These achievements resulted in tremendous interest in laser based atomic cooling methods and resulted in a Nobel prize awarded to Steven Chu, Claude Cohen-Tannoudji, and William D. Phillips in 1997.

\subsection{Laser Cooling as an Enabling Tool}

Laser cooling of atomic vapors has served as a technique which has enabled the study of more idealized physical systems, expanding and refining scientific knowledge in a vast amount of areas. Laser cooling made possible the creation and study of Bose-Einstein condensates (BEC) of dilute atomic vapors [13-15]. It plays a central role in the latest generation of atomic fountain clocks [16] and has made possible the creation of optical frequency standards [17]. The study of cold atomic collisions, nonlinear optical effect and basic quantum mechanical phenomena have greatly benefited from the ability to cool atoms. Precision spectroscopic investigations of the energy structure of atoms have benefited tremendously from the ability to trap and cool atomic 
samples by allowing for very long interaction times and a suppression of the Doppler effect (including time dilation). Cooled atoms with sufficiently long deBroglie wavelengths are of use in atom interferometers which can function as accelerometers, rotation sensors, gravimeters, and gradiometers. While this list is in no way exhaustive, it is merely to justify the incredibly important contribution laser cooling has provided to the scientific community.

\subsection{Limitations of Laser Cooling}

While the contributions of atomic laser cooling are substantial, no technique is without limitation. The major limitation of established atomic laser cooling techniques is that they rely on the transfer of momentum from photon to atom to work. This seemingly trivial statement places quite stringent requirements on atomic species amenable to these techniques. As a first step in seeing why, consider the number of scattering events necessary to stop a room temperature ${ }^{87} \mathrm{Rb}$ atom with photons resonant with its $\mathrm{D}_{2}$ transition. We can approximate this number as $m \bar{v} / \hbar k \approx 45,000$ where $m$ and $\bar{v}$ are the mass and velocity of a ${ }^{87} \mathrm{Rb}$ atom at $300 K$ and where $k=2 \pi / 780 \mathrm{~nm}$. From this consideration it is clear that a very large number of photon scattering events are necessary to stop a room temperature atom. In order to scatter such a large number of photons in an experimentally realizable way the atom must possess an effective two-level structure, depicted in Fig.1.1, that is accessible with a sufficiently intense available laser source.

The reason an effective two-level atom is required for laser cooling is 


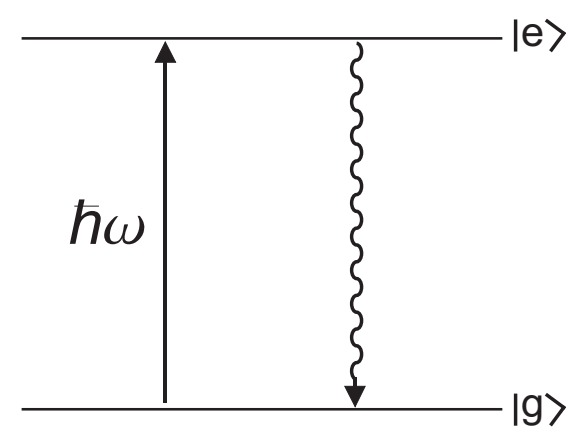

Figure 1.1: The energy level structure of a two-level atom. An atom initially in the ground state $|g\rangle$ is transfered by a resonant photon into the excited state $|e\rangle$. Once there it can only decay back into the ground state $|g\rangle$.

clear. In a typical laser cooling experiment atoms are initially in the ground state $|g\rangle$ and they are illuminated by a single frequency which is tuned below the atomic resonance. After absorbing a photon, atoms are excited into the upper state $|e\rangle$. If the incident field is not sufficiently intense to cause stimulated emission to dominate (as is the case when laser cooling), then the atom will spontaneously decay back into the ground state after a time $(\tau \propto 1 / \Gamma)$ characteristic of the transition. After it decays back into the ground state $|g\rangle$ it is again resonant with the laser beam and ready to scatter again. A twolevel structure ensures the continuation of this closed cycle. If, however, the atomic energy level structure is more complex then a second and detrimental possibility arises. After being excited into the upper state $|e\rangle$, the atom can decay to two different levels as shown in Fig.1.2. If it decays to $|g\rangle$, then it will again be resonant with the applied field and it will scatter again. If, however, it decays to the level $|h\rangle$, it will not be resonant with the applied field and will 


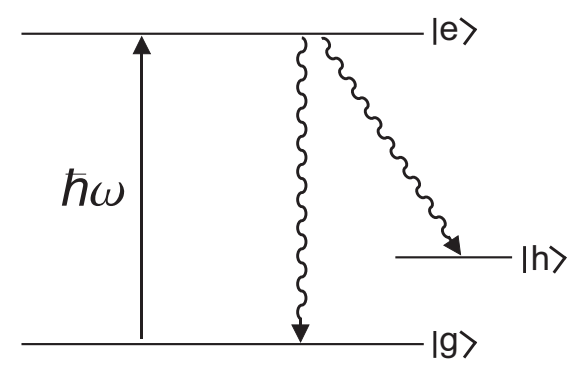

Figure 1.2: The energy level structure of a three-level atom. An atom initially in the ground state $|g\rangle$ is transfered by a resonant photon into the excited state $|e\rangle$. Once there it has two possible decay channels in contrast to the two-level system.

be removed from the cooling cycle. Because a very large number of scattering events are necessary to cool an atom, even a small probability of decaying into a state other than $|g\rangle$ is detrimental to the process.

Clearly no atom is truly two-level. Even hydrogen, the simplest atom, has a rich energy level structure [18]. Of course, this fact has not rendered laser cooling unworkable. Experimentally it means that one has to add additional "repumping" laser beams to reintroduce atoms into the cooling cycle after they have decayed to a state non-resonant with the cooling laser beam. For atoms with relatively simple energy structures such as alkali metals, suitable schemes have been developed with great success. With somewhat more effort many Noble gases have been laser cooled by first placing them into a metastable state. Additionally, through extreme efforts some alkaline earth metals as well as a handful of other atoms have been cooled. But for the vast majority of atoms and for all molecules laser cooling has not been an experimentally 
realizable method of cooling. Surprisingly, even hydrogen has evaded laser cooling, not because it lacks a suitable effective two-level structure, but rather due to the lack of an available laser source at $121 \mathrm{~nm}$.

The goal of the work detailed in this dissertation was to develop and demonstrate a more general cooling method that could potentially be applied to the vast majority of atoms in the periodic table.

\subsection{Introduction to Single-Photon Cooling}

Single-photon cooling differs fundamentally from established laser cooling techniques because it does not rely on the transfer of momentum from photon to atom to cool. Rather, cooling is achieved through the direct reduction of the entropy of an atomic ensemble. This feature of single-photon cooling sidesteps the major limiting factor of established laser cooling techniques allowing it to be potentially applied to a much larger portion of the periodic table, as well as molecules. To justify these claims this section will discuss, in general terms, the concept of single-photon cooling including, for pedagogical reasons, its origin and development. A more detailed description of the specific implementation of this cooling technique to a sample of ${ }^{87} \mathrm{Rb}$ will be deferred until Ch. 4 .

The original motivation for the development of single-photon cooling came from the field of plasma physics, where it was shown that an efficient asymmetric barrier for electrons or ions could be produced by subjecting the magnetically trapped species to a ponderomotive potential in the radio fre- 
quency regime [19]. This then led to the question of whether an analogous asymmetric barrier could be constructed for neutral atoms, and furthermore, whether such a device could be used to cool an atomic sample.

The answer to both of these questions was yes. In December 2004 and February 2005, papers were published outlining methods of producing asymmetric barriers optically for neutral atoms. While conceived at nearly the same time, these two methods were arrived at independently and differ from each other in several ways.

In a paper authored by A. Ruschhaupt and J.G. Muga [20], an optical asymmetric barrier was considered in the context of atomic control but not cooling.. It discussed a possible implementation of an "atom diode," a device that rectifies the motion of neutral atoms by letting ground-state atoms pass in one direction but not in the opposite direction. This process relied on stimulated Raman adiabatic passage (STIRAP) and only worked over a limited atomic velocity range. It required the use of three lasers: two to achieve the adiabatic transfer and the third to reflect the ground state atoms. While interesting for its use in atomic control, this paper did not consider using the diode for atomic cooling. These authors later collaborated with M.G. Raizen on a paper describing a version of the atomic diode which used a "quenching" beam to improve performance [21].

Independent work came from our group and also described a method of constructing an asymmetric one-way barrier for neutral atoms [22]. The details of the proposal differed from that of A. Ruschhaupt and J.G. Muga 
and our current implementation in ${ }^{87} \mathrm{Rb}$, but the enduring contribution of this paper was that it considered such a device in the context of atomic cooling.

Consider for the moment, without an explicit description of its construction, a one-way barrier for neutral atoms. Such a "one-way-wall" allows atoms to pass through it from one direction, but reflects them when they come from the opposite direction, as shown in Fig. 1.3. The pertinent ques-

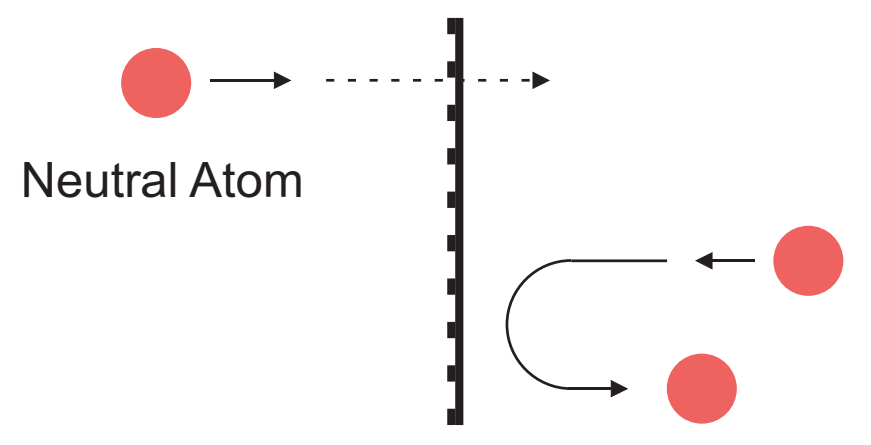

Figure 1.3: Depiction of a hypothetical one-way-wall for neutral atoms. While this wall allows atoms to transmit when traveling from left to right, it reflects atoms traveling from right to left.

tion becomes if such a one-way-wall can be used to cool an atomic ensemble. A simple theoretical arrangement utilizing a one-way-wall which clearly indicated that cooling is possible is given in Fig. 1.4. There a one-way-wall has been placed in a 1-D hard walled box containing an ensemble of thermal atoms. As the atoms explore the box they accumulate on one side of the one-way-wall. The atoms become confined to a small portion of the box of length $l_{2}$, which they originally filled, thus the ensemble of atoms has had its spatial spread $\sigma_{x}$ reduced. If we assume that no corresponding increase in the momentum 


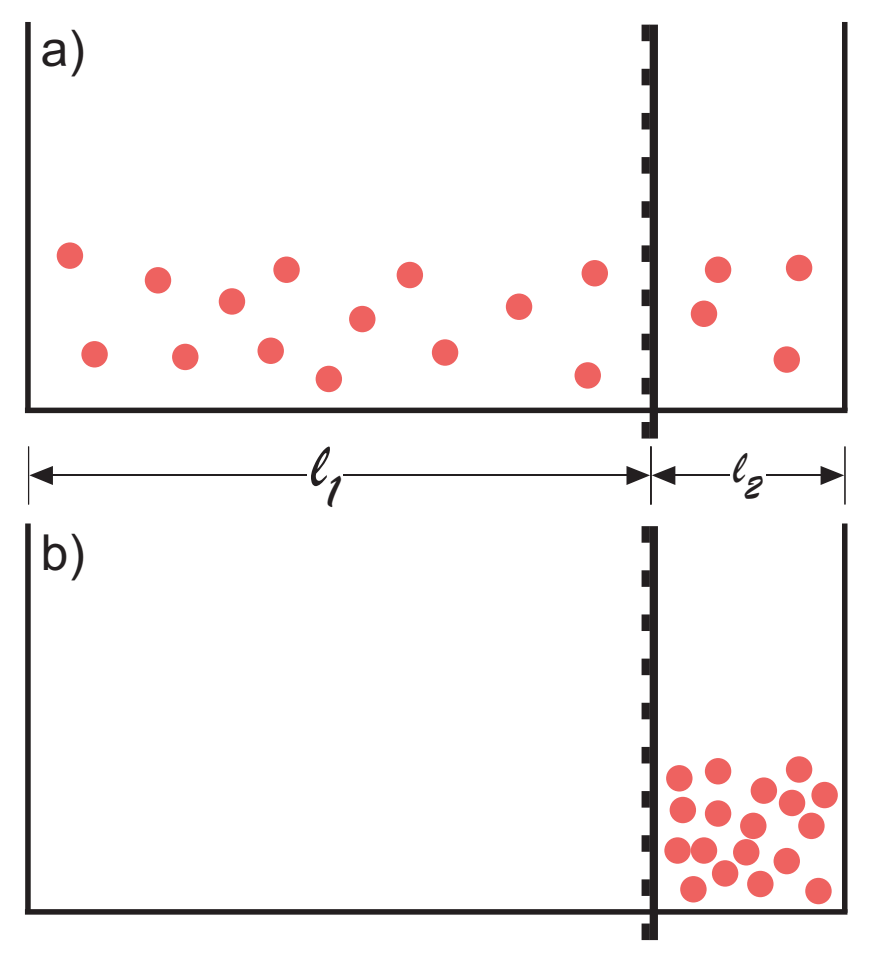

Figure 1.4: Depiction of a simple, hypothetical one-way-wall cooling scheme. (a) A one-way-wall is placed into a confined ensemble of atoms. (b) After sufficient time the atoms have explored the box and have accumulated on one side of the one-way-wall.

distribution $\sigma_{p}$ has taken place due to the action of the one-way-wall, then phase-space compression of the atomic ensemble has been accomplished with this simple arrangement. Here phase-space refers to the product of the widths of the spatial and momentum distributions $\rho \sim \sigma_{x} \sigma_{p}$.

A compression in phase-space can be used to reduce the temperature of a sample through adiabatic expansion. In this case one can imagine adiabatically moving the one-way-wall to the left in Fig. 1.4 to achieve this. If we 
assume that $l_{1}>>l_{2}$ and that the action of the one-way-wall heats the ensemble only a negligible amount then the phase-space compression $C$ achieved is given simply by

$$
C \equiv \frac{\rho_{\text {after }}}{\rho_{\text {before }}} \approx \frac{l_{1}}{l_{2}}
$$

While instructive, this simple example is clearly unphysical. The action of the barrier violates time reversal symmetry! Additionally, we have gotten something, namely atomic cooling, for nothing - in clear violation of the second law of thermodynamics. The resolution of these troubling facts comes from recognition that any physical realization of an atomic one-way-wall must affect the atoms beyond simply transmitting or reflecting them. For the action of the one-way-wall as presented thus far to obey time reversal symmetry the atoms on the left of the wall must be different in some way from the atoms on the right of the wall. Additionally, the decrease in entropy produced through the action of the one-way-wall must be compensated for by an increase in entropy elsewhere if this scheme is to obey the second law of thermodynamics. Indeed, in the physical realization of this cooling process presented in this dissertation the one-way-wall labels the atoms on either side of the barrier by placing them into distinct hyperfine states through an irreversible spontaneous scattering process of a single photon as atoms transit the barrier. This scattering process is of course the origin of the name "single-photon cooling." Much more will be said of this in Ch. 4 where a complete description of the cooling process applied to ${ }^{87} \mathrm{Rb}$ is described.

Now consider a second example, a slight variation of the first, which 
demonstrates the cooling power of a one-way-wall process in a geometry more similar (but not identical) to that used in the actual experiment. In this example, depicted in Fig. 1.5, an atom is placed into an external, conservative potential. This potential in indicated by the "V"-shape in each sub-figure (a-e). As shown in Fig. 1.5(a) the one-way-wall is initially positioned such that the atom does not have sufficient energy to reach it. To initiate cooling, the one-way-wall is slowly swept towards the center of the confining external potential. If this is done slowly enough, the atom will first encounter the one-way-wall near its turning point, where it has exchanged most of its kinetic energy for potential energy. The sweep must be done slowly because as the oneway-wall is swept its intersection point with the confining potential decreases in energy at a rate $\dot{E}$. If the oscillation time of the trapped atom in the confining potential is given by $T_{\text {atom }}$ then the average residual kinetic energy $K_{\text {res }}$ retained by the atom after transiting the one-way-wall due to the motion of the one-way-wall is

$$
K_{\text {res }}=\frac{1}{2} \dot{E} T_{\text {atom }}
$$

Experimental factors force one to compromise between a small $K_{\text {res }}$ and a reasonable sweep duration. After transiting the one-way-wall the atom is captured with little residual kinetic energy Fig. 1.5(c). According to our working definition of temperature given in Eq. 1.5 this atom has been cooled. As the one-way-wall then continues to sweep towards the center of the external confining potential one may expect that the atom will heat back up. This is not so. The work done on the atom by the one-way-wall as it moves is equal to 

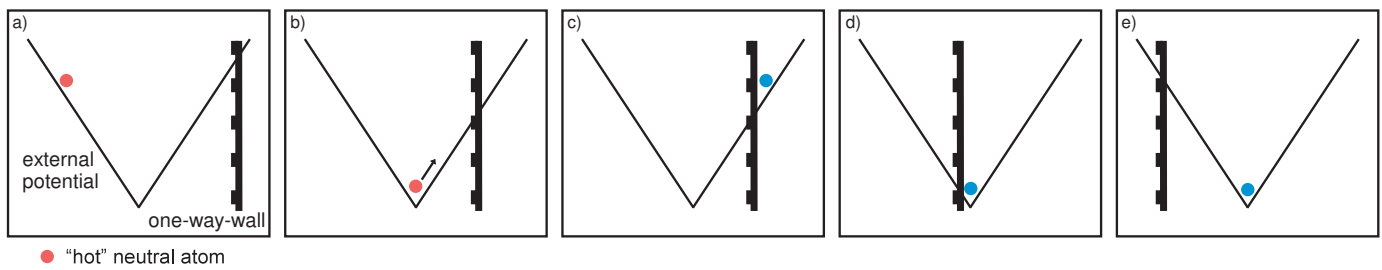

"cold" neutral atom

Figure 1.5: (a) An atom is placed in an external confining potential along with a one-way-wall which is initially placed such that the atom does not have sufficient energy to reach it. (b) The one-way-wall is slowly swept towards the trap center, so that the atom first encounters it at its turning point. (c) The one-way-wall continues to sweep in, eventually returning the cold atom to the center of the external confining potential (d). (e) The one-way-wall moves away from the region containing the atoms.

the increase in potential energy of the atom [21]. This is completely analogous to the fact that the work done by an ideal elevator is equal to the change in the potential energy of its occupants. After sweeping through the center of the external confining potential the atom remains trapped at its center, but is now cold [Fig. 1.5(d)]. The one-way-wall continues its sweep out of the region of the atom [Fig. 1.5(e)].

Because this scheme is fundamentally a single atom process, it works equally well on an ensemble of non-interacting atoms. To work on an ensemble the one-way-wall must be initially positioned such that the most energetic atom in the group does not have sufficient energy to reach it (Fig. 1.6). The rate at which the one-way-wall is swept must be slow enough to allow all atoms in the ensemble to reach it with negligible kinetic energy. Because this technique operates at the single particle level it does not rely on a specific form of inter- 

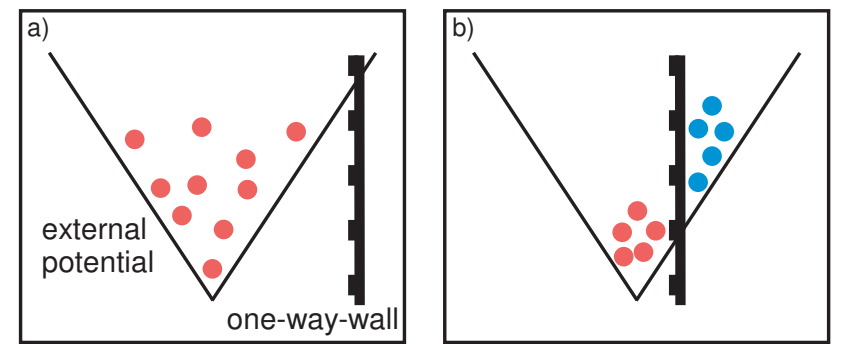

- "hot" neutral atom

"cold" neutral atom

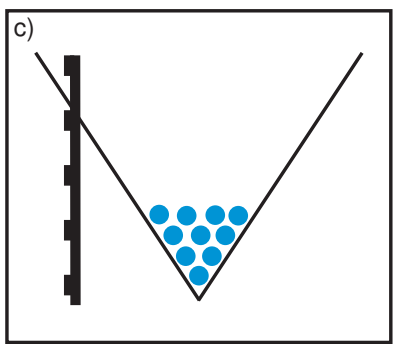

Figure 1.6: (a) A non-interacting atomic ensemble is placed in an external confining potential along with a one-way-wall which is initially placed such that none of the atoms have sufficient energy to reach it. (b) The one-waywall is slowly swept towards the trap center, capturing atoms as it encounters them at their turning points. (c) The one-way-wall continues its sweep, eventually capturing all the atoms and returning them to the center of the external confining potential with reduced kinetic energy.

particle interactions to work, as does evaporative cooling [23]. This feature further generalizes and extends its potential applicability.

This section served as an introduction to the concept of single-photon cooling. In summary, this technique begins by trapping an atomic ensemble in a conservative potential. Then, a one-way-wall for atoms is positioned in the wings of the trap. Cooling is initiated by slowly sweeping the one-waywall towards the center of the conservative potential. The sweep must be slow compared to the oscillation frequency of the trapped atoms, so that they first encounter it near a turning point. After transmitting through the one-waywall near a turning point atoms become trapped with little kinetic energy. The atoms become trapped because they are placed into a different internal state 
by the one-way-wall through an irreversible step. The one-way-wall continues to sweep until all atoms have encountered it, been cooled and returned to the center of the conservative potential. This general technique is applied, for the first time, to the specific case of cooling magnetically trapped ${ }^{87} \mathrm{Rb}$, and details of its specific implementation and results are given in Ch. 4 .

\subsection{Single-Photon Cooling as a Maxwell's Demon}

In 1867 James Clerk Maxwell proposed the notion of a "very observant and neat-fingered being" and wondered if under certain circumstances it could possibly violate the second law of thermodynamics [24]. He imagined such a creature, later given the honorable designation of a "Maxwell's Demon" by Lord Kelvin [25], as operating a trap door separating two halves of a vessel filled with gas molecules initially in thermal equilibrium. He argued that if the demon sensed the velocity of molecules at they approached and directed fastmoving molecules into one half of the vessel and slow-moving molecules into the other half it would cause a temperature differential "without expenditure of work." A second version of the demon would simply let molecules pass into one half of the vessel but never out of it, thus developing a pressure differential. Such apparent violations of the second law of thermodynamics spurred intense interest and research into "exorcising" such demons, in other words demonstrating that they do not violate any microscopic or macroscopic law of physics.

One enduring notion, originally proposed by Szilard [26], and later 
worked on by Brillouin [27-29], identified the information obtained by the demon, which it used to determine the appropriate action of the trap door, as having physical entropic content. This concept effectively exorcised such Maxwell Demons because the entropy associated with the information gathered by the demon is never less than the reduction of entropy due to the demon's actions. This notion of information carrying entropy has become a key concept in information theory ever since [30-33]. Despite the fact that the work done by Szilard and others demonstrated that such processes do not violate any physical law, any proposal or experiment in this vein has been continued to be called a Maxwell's Demon.

Single-photon cooling is an optical realization of a Maxwell's Demon. An atomic ensemble confined in a conservative potential is directly analogous to the 'gas in a vessel.' The demon analog, however, is not simply the one-waywall alone. Rather, it is the combination of the one-way-wall and its carefully selected slow sweep through the trapped atomic ensemble. The information gathered by the demon is the single photon spontaneously scattered by each atom as it transits the barrier. To make things a little more concrete, consider the action of the single-photon cooling process on a non-interacting atomic ensemble with the well defined energy distribution $f_{E}$ defined such that

$$
n(E)=N f_{E} d E
$$

where $N$ is the total number of atoms, and $n(E)$ is the number of atoms with energy between $E$ and $E+d E$. Figure 1.7(a) shows such an energy distribution 
$f_{E}$. Furthermore, assume that the conservative confining potential and the position of the one-way-wall is well known at all times during the cooling process. As shown in Fig. 1.7(b), as the one-way-wall is slowly swept towards
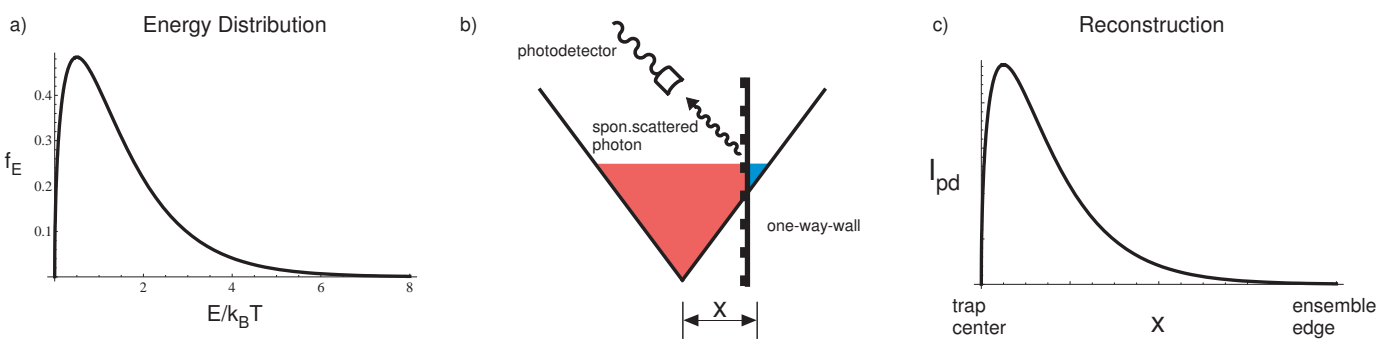

Figure 1.7: (a) The initial energy distribution of a non-interacting atomic ensemble. (b) This ensemble is placed into a conservative trapping potential. As the one-way-wall sweeps through the ensemble it encounters the most energetic atoms first, which spontaneously scatter photons as they transit the barrier. A photodetector monitors the scattered light. (c) A plot of the photodetector signal $I_{p d}$ as a function of the position of the one-way-wall $x$ reconstructs the original energy distribution of the ensemble.

the center of the confining potential, it first encounters the most energetic atom at a position where the atom has converted (nearly) all of its energy into potential energy. When the atom transits the one-way-wall it undergoes an irreversible process which involves the scattering of a spontaneous photon. Detection of this photon reveals the atom's initial energy through knowledge of the location of the one-way-wall $x$ at the time of scatter and the shape of the confining potential. By detecting and recording each spontaneously scattered photon as a function of the one-way-wall's position with respect to the confining potential, the initial energy distribution can be reconstructed, as shown in Fig. 1.7(c). Of course, this sweep also has the effect of cooling 
the atomic ensemble. Not surprisingly and as shown in [34], the information entropy content carried away by the scattered photons is equal to the reduction in the entropy of the cooled atomic ensemble.

What is really striking is how extremely efficient the single-photon cooling process is in using information entropy to cool. In the ideal case described above it is perfectly efficient; the reduction in entropy of the atomic ensemble equals the information entropy carried away by the spontaneously scattered photons. When compared to other established atomic laser cooling techniques, where the increase in photon entropy is typically several orders of magnitude higher than the decrease in the entropy of the atomic ensemble cooled [35], we see just how truly remarkable this process is. Additionally, single-photon cooling is a passive technique in the sense that one need not monitor the scattered photons at all for the process to work. In contrast, stochastic cooling methods, which like single-photon techniques scatter photons which carry information related the ensemble's properties, must actively feedback on the data gathered from scattered photons to operate $[36,37]$.

\subsection{A Note on Units}

A brief discussion of the terminology and units used throughout is warranted. Of particular note is the loose manner in which the term "temperature" is used in the laser cooling community and indeed in this text. The concept of temperature is defined in thermodynamics as a property of a closed system in thermal equilibrium with its surroundings $[38,39]$. In typical atomic 
cooling experiments, which are performed in ultrahigh vacuum environments, the atomic ensemble being cooled is necessarily trapped optically and/or magnetically away from any thermal reservoir (e.g. the vacuum chamber walls). While the atoms may be in a steady-state situation, they are not necessarily in thermodynamic equilibrium. Despite this fact it is often convenient to define the temperature of an ensemble of atoms as

$$
\frac{1}{2} k_{B} T=\left\langle E_{k}\right\rangle
$$

where $k_{B}$ is Boltzmann's constant and $\left\langle E_{k}\right\rangle$ is the average kinetic energy of an ensemble in 1-D.

It is also worth noting that throughout this text, cooling is used not in the weak sense, as simply a reduction in temperature, but in a much more meaningful way - as an increase in phase-space density. This distinction must be made because an atomic vapor can always be trivially cooled through adiabatic expansion, but this sort of "cooling" is rarely useful in experiments. For most atomic physics experiments such as Bose-Einstein condensation or spectroscopic investigations of atomic energy structures, an increase in phase-space density is what is helpful.

As mentioned above, atoms in these sorts of experiments are typically trapped optically and/or magnetically and so a trapping depth is commonly reported. In this context, trapping depth refers to the potential energy depth of the trap (see Fig. 1.8). Atoms with total energy less than the trap depth are considered trappable while those with energy greater than the trap depth 
are untrappable.

The SI unit for energy is the Joule, however it is often more convenient in atomic cooling experiments to divide this value by Boltzmann's constant to report a temperature. This has the benefits of allowing a simple comparison between trap depth and an atomic ensemble's temperature as well as putting the values reported in a more agreeable form. For example, typical optical trap depths reported in this dissertation are on the order of $10 \mu K$, which corresponds to a value of $1.4 \times 10^{-28} J$ in SI units.

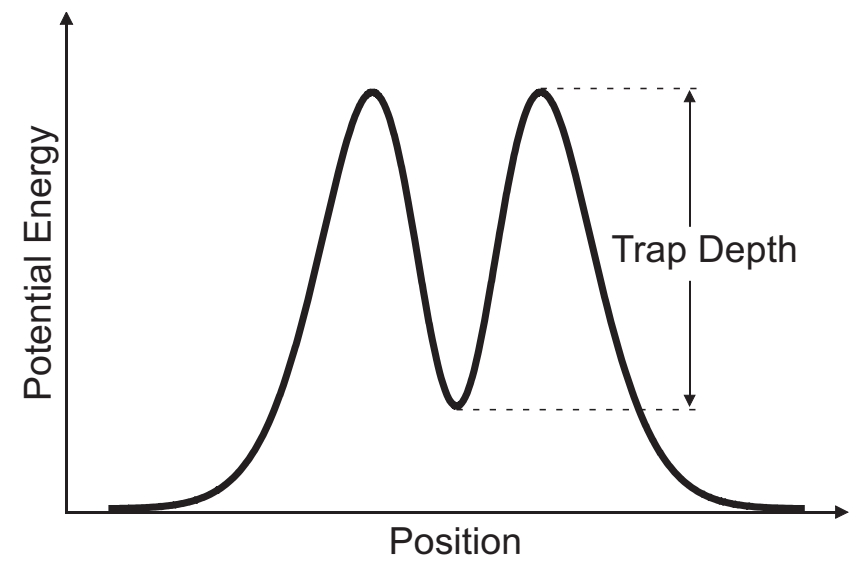

Figure 1.8: Trap depth in the context of atomic cooling. Experimentally the potential energy surface shown in the figure is typically due to the interaction of an atom with an electric, magnetic or optical field.

For completeness, a set of units used extensively throughout the laser cooling community is presented. Each unit represents a value or temperature of significant importance. The first such value is the first order Doppler shift

$$
\Delta \omega_{D}=\frac{v_{a t o m}}{c} \omega_{L} .
$$


This represents a shift in the frequency of an incident light field in the frame of an atom in motion with respect to the light source. Here $\Delta \omega_{D}$ is the shift in frequency, $\omega_{L}$ is the rest frame frequency and $v_{a t o m}$ is the component of atomic velocity parallel to the wavevector of the incident light field. This relation is valid for $v_{\text {atom }} / c<<1$, where $c$ is the vacuum speed of light, and is sufficient for most cases encountered when optically cooling an atomic sample. There are also several atomic velocities and corresponding temperatures of interest, I will discuss them in order of descending energy.

The highest atomic velocity of significance is known as the cooling velocity $v_{c}$. It corresponds to the velocity of an atom such that its Doppler shift is comparable to the natural linewidth $\Gamma$ of the transition being used to cool the atom. This value represents the velocity at which an atom is just at the edge of possible photon absorption from a narrow band laser beam which is resonant with the transition of interest. This velocity is given by

$$
v_{c} \equiv \frac{\Gamma}{k_{L}}
$$

where $k_{L}$ is the wavevector of the incident light in the rest frame. For example, $v_{c} \approx 4.5 \mathrm{~m} / \mathrm{s}$ in ${ }^{87} \mathrm{Rb}$, the element used in all experiments discussed in this dissertation. The temperature corresponding to this velocity is given by

$$
\Delta T_{c} \equiv \frac{m \Gamma^{2}}{k_{B} k_{L}^{2}},
$$

where $m$ is the atomic mass.

The next value of interest corresponds to the energy associated with 
the atomic transition and is called the Doppler temperature

$$
T_{D} \equiv \frac{\hbar \Gamma}{2 k_{B}}
$$

This value represent a lower limit, the Doppler limit, in some laser cooling techniques. For ${ }^{87} \mathrm{Rb}$ the Doppler temperature is $\approx 146 \mu \mathrm{K}$.

The last important value is the recoil velocity $v_{r}$. It corresponds to the velocity change an atom undergoes from absorbing or emitting a single resonant photon. It is given by

$$
v_{r} \equiv \frac{\hbar k_{L}}{m}
$$

and has a value of $\approx 5.8 \mathrm{~mm} / \mathrm{s}$ in ${ }^{87} \mathrm{Rb}$. The temperature associated with this velocity, the recoil temperature $T_{r}$ is given by

$$
T_{r} \equiv \frac{\hbar^{2} k_{L}^{2}}{m k_{B}}
$$

and has a value of $\approx 362 n K$ in ${ }^{87} \mathrm{Rb}$. This temperature is seen as the lower limit to all atomic laser cooling methods, although there are a few clever schemes which circumvent it. 


\section{Chapter 2}

\section{Operational Concepts}

This chapter discusses many of the concepts necessary for a complete understanding of the experimental procedure and results presented in this dissertation. It begins with a general discussion of the element used during this experimental work, rubidium. A description of the source and size of energy level splittings resulting in the fine and hyperfine structure is given. The interaction of atoms with external magnetic fields is discussed, as well as how this interaction, known as the Zeeman effect, can be used to trap rubidium. A discussion of the interaction of atoms with external optical fields is given, both in the near and far off resonance limits. How these interactions lead to forces which can be used to cool and control external atomic degrees of freedom is explained. The frequency of applied optical fields must be precisely controlled and the method used to accomplish this, a feedback loop based on saturation absorption spectroscopy of a room temperature vapor cell, is discussed. Finally the two methods used in this dissertation to image atomic samples, optical absorption and atomic fluorescence are discussed. 


\section{$2.1 \quad$ Rubidium}

Rubidium $(\mathrm{Rb})$ is an alkali metal. Its common use in atomic experiments is due in part to its relatively simple energy level structure and its possession of an accessible effective cycling transition. The simplicity of the energy level structure is due to the fact that of Rb's 37 electrons only one is in the outermost shell. ${ }^{85} \mathrm{Rb}$ is the most abundant and only stable isotope of rubidium, however the experimental work performed in this dissertation was done entirely on ${ }^{87} \mathrm{Rb}$. The reason for this is that ${ }^{87} \mathrm{Rb}$ has a simpler hyperfine structure, the topic of the next section. ${ }^{87} \mathrm{Rb}$ has a relative natural abundance of $27.83(2) \%$ and decays to $\beta^{-}+{ }^{87} \mathrm{Sr}$ with a total disintegration energy of $0.283 \mathrm{MeV}$ [40]. While unstable, the nuclear lifetime is $4.88 \times 10^{10} \mathrm{yr}$ [40] making this form of rubidium effectively stable over the duration of our experiment. The mass of ${ }^{87} \mathrm{Rb}$ was accurately determined from a Penning trap measurement to be $m=1.443160648(72) \times 10^{-25} \mathrm{~kg}$ [41]. The vapor pressure of solid $\mathrm{Rb}$ is an important experimental quantity because it dictates the loading rate into the first stage of our experimental procedure. This value can be taken from a vapor-pressure model for solid Rb given by [42], which is

$$
\log _{10} P_{v}=2.881+4.857-\frac{4215}{T}
$$

where $P_{v}$ is the vapor pressure in torr and $T$ is in $\mathrm{K}$. This model is specified to have an accuracy better than $\pm 5 \%$ from $298-550 \mathrm{~K}$. The ambient temperature surrounding our experimental chamber is approximately $295 \mathrm{~K}$ resulting in a vapor pressure of $2.9 \times 10^{-7}$ torr. More will be said about how this relates 
to the experimental sequence in Ch. 3. Table 2.1 summarizes the physical properties discussed here. A more complete tabulation and discussion of these properties is organized in an excellent reference [43].

\begin{tabular}{|l|c|c|}
\hline Atomic Number & $\mathrm{Z}$ & 37 \\
\hline Total Nucleons & $\mathrm{Z}+\mathrm{N}$ & 87 \\
\hline Relative Natural Abundance & $\eta\left({ }^{87} \mathrm{Rb}\right)$ & $27.83(2) \%$ \\
\hline Atomic Mass & $\mathrm{m}$ & $1.443160648(72) \times 10^{-25} \mathrm{~kg}$ \\
\hline Nuclear Spin & $\mathrm{I}$ & $3 / 2$ \\
\hline
\end{tabular}

Table 2.1: ${ }^{87} \mathrm{Rb}$ Physical Properties

\section{$2.2 \quad$ Fine and Hyperfine Structure}

This section reviews interactions leading to a splitting in atomic energy levels. First the fine structure is explored, followed by a discussion of the hyperfine structure in rubidium.

\subsubsection{Fine Structure}

The primary source of energy level splittings in atoms are the electrostatic attraction between the electrons and nucleus and the electrostatic repulsion between the individual electrons. The energy levels resultant from these interactions are known as the Bohr energy levels. The next most important contribution to energy level splittings in low $Z$ atoms are a result of relativistic effects. These effects are the source of the fine structure of atomic spectra. The fine structure energy level splittings are smaller than the Bohr energy level splittings by a factor of $\sim \alpha^{2}$. Here $\alpha$ denotes the fine structure 
constant which is given by

$$
\alpha=\frac{e^{2}}{\hbar c 4 \pi \epsilon_{0}} \approx \frac{1}{137}
$$

There are two causes of the fine structure splittings. The first is due to the coupling between the magnetic moment of the electrons and the effective magnetic field seen by the electrons due to their motion around the nucleus. The second cause is due to relativistic corrections to the kinetic and potential energy of the electrons. For Rb, the first contribution dominates and so I will focus solely on it.

The Hamiltonian describing the interaction between the outermost electron's magnetic moment and orbital angular momentum can be written as

$$
H_{f s}=A(\vec{L} \cdot \vec{S})
$$

where $A$ is a constant parameterizing the strength of the interaction, $\vec{S}$ is the spin of the electron and $\vec{L}$ is the orbital angular momentum of the electron.

To solve this Hamiltonian we introduce the total electron angular momentum $\vec{J}$, given by

$$
\vec{J}=\vec{L}+\vec{S}
$$

where by the triangle inequality the quantum number $J$ must lie in the range

$$
|L-S| \leq J \leq L+S
$$

The convention that the magnitude of $\vec{J}$ is $\hbar \sqrt{J(J+1)}$, and that the eigenvalue of $J_{z}$ is $m_{J} \hbar$ is being used. We next note that squaring both sides of 
Eq. 2.4 yields

$$
J^{2}=S^{2}+L^{2}+2 \vec{L} \cdot \vec{S}
$$

allowing us to rewrite the fine structure Hamiltonian (Eq. 2.3) as

$$
H_{f s}=\frac{A}{2}\left(J^{2}-S^{2}-L^{2}\right) .
$$

Because the energy shift of this Hamiltonian is small compared to the Bohr splittings, it can be introduced as a perturbation to those energy levels. Time independent perturbation theory allows us to write the shift in an energy level to first order due to this perturbation as

$$
\Delta E=\left\langle\Psi\left|H_{f s}\right| \Psi\right\rangle
$$

This notation introduces the question of what basis should be taken to make the evaluation of this matrix element the simplest. The coupling of $\vec{L}$ and $\vec{S}$ has caused $L_{z}$ and $S_{z}$ to be unconserved quantities so the uncoupled basis $\left|L, L_{z}, S, S_{z}\right\rangle$ is clearly not an eigenvector of Eq. 2.7. However, from inspection it is clear that elements of the coupled basis $\left|S, L, J, J_{z}\right\rangle$ are eigenvectors of the fine structure Hamiltonian and can be used to evaluate Eq. 2.7, immediately resulting in

$$
\Delta E=\frac{A \hbar^{2}}{2}[J(J+1)-S(S+1)-L(L+1)]
$$

The ground state configuration of ${ }^{87} \mathrm{Rb}$ is $[K r] 5 s$. This means that there is one unpaired electron and that this electron has no orbital angular momentum, therefore $S=1 / 2$ and $L=0$. This means that the only value $J$ can take is $J=1 / 2$. Evidently, the ground state is not split by $H_{f s}$ because 
only one value of $J$ is possible from this configuration. This single ground state term can be written compactly using Russel-Saunders notation as $5^{2} S_{1 / 2}$. The meaning of this term is as follows. The first number is the principle quantum number $n$ of the outer electron. The superscript represent $2 S+1$, and here since $S=1 / 2$ is 2 . The uppercase letter corresponds to the total orbital angular momentum $L$ such that $S=0, P=1, D=2, F=3 \ldots$ The subscript represents the value of the total electronic angular momentum $J$.

The configuration of the first excited state of ${ }^{87} \mathrm{Rb}$ is $[K r] 5 p$. Because in this state $L=1$ there are two possible values of $J, 1 / 2$ and $3 / 2$, leading to two possible terms $5^{2} P_{1 / 2}$ and $5^{2} P_{3 / 2}$. Since the value of $\Delta E$ depends on $J$ these two terms are split in energy giving rise to a fine-structure doublet. The two transitions $5^{2} S_{1 / 2} \rightarrow 5^{2} P_{1 / 2}$ and $5^{2} S_{1 / 2} \rightarrow 5^{2} P_{3 / 2}$ are known respectively as the $D_{1}$ and $D_{2}$ transitions. The $D_{1}$ transition is at $\approx 795 \mathrm{~nm}$ while the $D_{2}$ is at $\approx 780 \mathrm{~nm}$. Because these two transitions are easily resolved by many lasers they are typically treated separately. Indeed, the work done in this dissertation used the $D_{2}$ transition exclusively.

\subsubsection{Hyperfine Structure}

The hyperfine splitting of atomic energy levels is due to the interaction of the total electronic angular momentum $J$ with the total nuclear angular momentum $I$. As the name suggests this effect is even smaller than the fine structure splitting, reduced by the factor $\sim m_{e} / M_{P} \approx 1 / 1836$ which is the electron to proton mass ratio. As in the case of the fine structure, we can form 
the sum of the two coupled angular momentum vectors $J$ and $I$, in this case giving the total atomic angular momentum $F$

$$
\vec{F}=\vec{J}+\vec{I}
$$

which is similarly constrained by the triangle inequality to have a magnitude with the range

$$
|J-I| \leq F \leq J+I
$$

To get a feeling for how this works, consider the ground state of ${ }^{87} \mathrm{Rb}$ which has the single term $5^{2} S_{1 / 2}$. Because $I=3 / 2$ and $J=1 / 2$, Eq. 2.11 indicates that $F$ can take on two values: 1 or 2 . In the excited state of the $D_{2}$ transition the term is $5^{2} P_{3 / 2}$ so $F$ can take on the values $0,1,2$, or 3 .

The Hamiltonian describing the interaction between the total electronic angular momentum $J$ and the total nuclear angular momentum $I$ is given by $[44-47]$

$$
H_{h f s}=A_{h f s} \vec{I} \cdot \vec{J}+B_{h f s} \frac{3(\vec{I} \cdot \vec{J})^{2}+\frac{3}{2}(\vec{I} \cdot \vec{J})-I(I+1) J(J+1)}{2 I(2 I-1) J(2 J-1)},
$$

where the interaction between $J$ and the magnetic dipole moment and electric quadrupole moment of the nucleus has been included. Higher order terms resulting from interactions with higher order nuclear moments have been neglected in this Hamiltonian because experimental measurements are not sufficiently accurate to assign a non-zero contribution to them. In Eq. 2.12, $A_{h f s}$ is the magnetic dipole constant and $B_{h f s}$ is the electric quadrupole constant, 
the latter of which only applies to the excited manifold of the $D_{2}$ transition and not to levels with $J=1 / 2$.

This Hamiltonian can be solved in a manner analogous to that used in the case of fine structure splitting. We note that by squaring both sides of Eq. 2.10 one can write,

$$
\vec{J} \cdot \vec{I}=\frac{1}{2}\left(F^{2}-J^{2}-I^{2}\right)
$$

This can be used in Eq. 2.12 to solve for the shifts in energy levels due to this interaction. Again we note that the coupling of $I$ and $J$ causes the uncoupled basis $\left|I, I_{z}, J, J_{z}\right\rangle$ to no longer be an eigenvector of the Hamiltonian describing the atom. But as before, simple inspection revels that $\left|I, J, F, m_{F}\right\rangle$ is an eigenvector of Eq. 2.12. Taking this as the basis in evaluating the perturbation due to the hyperfine Hamiltonian results in energy splittings given by

$$
\Delta E_{h f s}=\frac{1}{2} A_{h f s} K+B_{h f s} \frac{\frac{3}{2} K(K+1)-2 I(I+1) J(J+1)}{4 I(2 I-1) J(2 J-1)},
$$

where

$$
K=F(F+1)-I(I+1)-J(J+1)
$$

is introduced for notational convenience.

The nuclear moment constants for the ${ }^{87} \mathrm{Rb} D_{2}$ line are given in Table 2.2. The ground state value was taken from a precise atomic fountain measurement [48], while the excited state values were measured using a heterodyne technique between two ultra stable lasers referenced to atomic ${ }^{87} \mathrm{Rb}$ [49]. 


\begin{tabular}{|c|c|}
\hline$A_{5^{2} S_{1 / 2}}$ & $h \cdot 3.417341305452145(45) \mathrm{GHz}$ \\
\hline$A_{5^{2} P_{3 / 2}}$ & $h \cdot 84.7185(20) \mathrm{MHz}$ \\
\hline$B_{5^{2} P_{3 / 2}}$ & $h \cdot 12.4965(37) \mathrm{MHz}$ \\
\hline
\end{tabular}

Table 2.2: ${ }^{87} \mathrm{Rb} D_{2}$ Transition Hyperfine Structure Constants

The hyperfine structure of the ${ }^{87} \mathrm{Rb} D_{2}$ transition is shown in Fig. 2.1. The energy shifts in this figure are given by Eq. 2.14 with the constants reported in Table 2.2. Each shift is with respect to the transition's center of gravity value, indicated by a horizontal dashed line. 

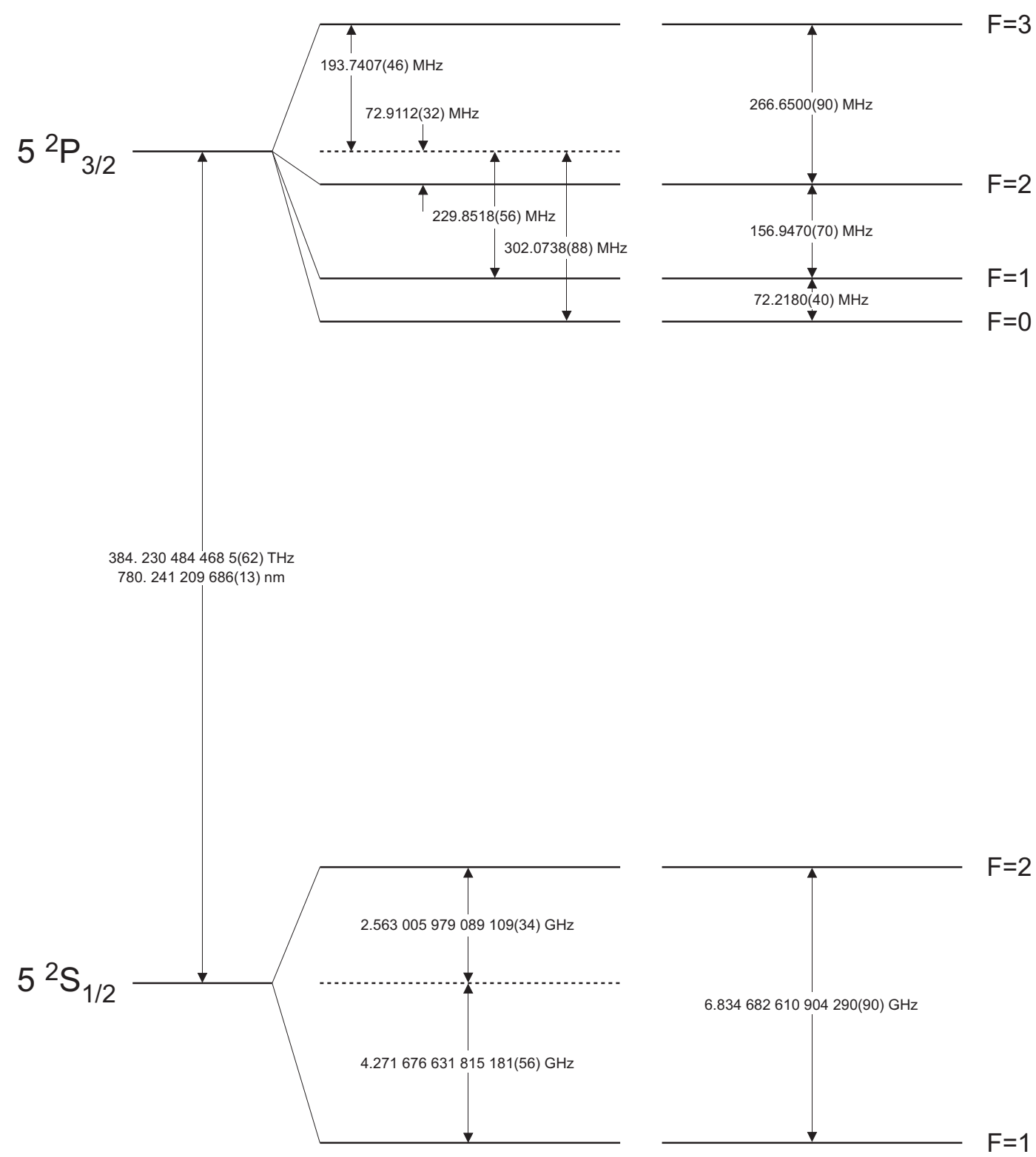

Figure 2.1: ${ }^{87} R b D_{2}$ Transition Hyperfine Structure. 


\subsection{The Zeeman Effect}

The Zeeman effect refers to a shift in an atomic energy level due to the presence of an external magnetic field. Each of the hyperfine levels $F$ contains $2 F+1$ magnetic sublevels, labeled by $m_{F}$, which correspond to different projections of the total atomic angular momentum along the quantization axis, here taken to be along $\hat{z}$. These states are represented by the vectors $\left|F, m_{F}\right\rangle$ and are eigenvectors of the operators $F^{2}$ and $F_{z}$.

$$
\begin{gathered}
F^{2}\left|F, m_{F}\right\rangle=\hbar^{2} F(F+1)\left|F, m_{F}\right\rangle \\
F_{z}\left|F, m_{F}\right\rangle=\hbar m_{F}\left|F, m_{F}\right\rangle
\end{gathered}
$$

In the absence of an external magnetic field, sublevels of a common hyperfine state $F$ are degenerate. Application of a magnetic field lifts this degeneracy.

The total atomic magnetic moment of the atom is the sum of the electronic and nuclear moments

$$
\vec{\mu}_{\text {atom }}=-\mu_{B}\left(g_{J} \vec{J}+g_{I} \vec{I}\right),
$$

where $\mu_{B}=h \cdot 1.399624604(35) \mathrm{MHz} / \mathrm{G}$ is the Bohr magneton and $g_{J}$ and $g_{I}$ are the total electronic angular momentum and nuclear "g-factors" which arise from projections of $\vec{J}$ and $\vec{I}$ along $\vec{F}$. The Hamiltonian for the interaction of $\vec{\mu}_{\text {atom }}$ with an external magnetic field is $[50,51]$

$$
H_{Z E}=-\vec{\mu}_{\text {atom }} \cdot \vec{B}
$$


Pluging Eq. 2.18 into this Hamiltonian yields

$$
H_{Z E}=\mu_{B}\left(g_{J} \vec{J}+g_{I} \vec{I}\right) \cdot \vec{B}
$$

If the interaction of the atom with an external field described by this equation is small compared to the hyperfine splitting then $F$ is a good quantum number. A useful method of visualizing this situation is provided by the vector model of angular momentum. This particular situation is depicted in Fig. 2.2, which illustrates that the coupling of $\vec{J}$ and $\vec{I}$ can be interpreted as the sum of these two vectors precessing about the total atomic angular momentum vector $\vec{F}$. In turn $\vec{F}$ precesses about the external magnetic field. As $\vec{J}$ and $\vec{I}$ precess

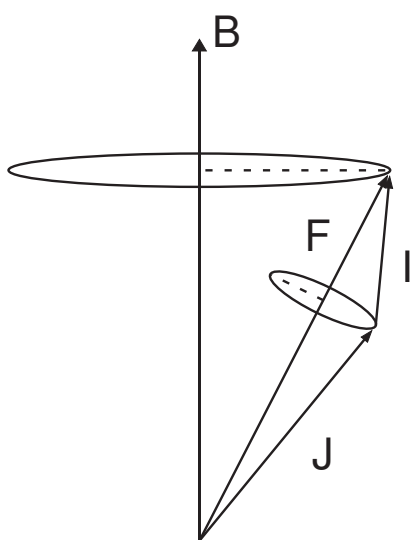

Figure 2.2: Vector model of the hyperfine interaction. The coupling of $\vec{J}$ and $\vec{I}$ can be interpreted as the sum of these two vectors precessing about the total atomic angular momentum vector $\vec{F}$. Similarly, but at a much slower rate, $\vec{F}$ precesses about the external magnetic field. As $\vec{J}$ and $\vec{I}$ precess about $\vec{F}$, their projections along $\vec{B}$ (the quantization axis) change in time. Therefore the quantum numbers associated with these projections $J_{z}$ and $I_{z}$ (assuming that $\mathrm{B}$ is along $\hat{z}$ ) are not good quantum numbers. In contrast, the projection of $\vec{F}$ along $\vec{B}$ is constant in time and so $F_{z}$ is a good quantum number. 
about $\vec{F}$, their projections along $\vec{B}$ (the quantization axis) changes in time. Therefore the quantum numbers associated with these projections $J_{z}$ and $I_{z}$ (assuming that $\mathrm{B}$ is along $\hat{z}$ ) are not good quantum numbers. In contrast, the projection of $\vec{F}$ along $\vec{B}$ is constant in time and so $F_{z}$ is a good quantum number. Therefore the Zeeman Hamiltonian (Eq. 2.20) can be written in a more useful form, in this case, by taking the projection of $\vec{J}$ and $\vec{I}$ along $\vec{F}$

$$
H_{Z E}=\mu_{B}\left(g_{J} \frac{\langle\vec{F} \cdot \vec{J}\rangle F \cdot B}{F(F+1)}+g_{I} \frac{\langle\vec{F} \cdot \vec{I}\rangle F \cdot B}{F(F+1)}\right),
$$

where the terms in the angled brackets refer to an expectation value. This can be written more compactly as

$$
H_{Z E}=\mu_{B} g_{F} F_{z} B
$$

where $\mathrm{B}$ has been taken along $\hat{z}$ and $g_{F}$ is given by

$$
g_{F}=g_{J} \frac{\langle\vec{F} \cdot \vec{J}\rangle}{F(F+1)}+g_{I} \frac{\langle\vec{F} \cdot \vec{I}\rangle}{F(F+1)}
$$

The expectation values can be written in a more illuminating manner; for example $\langle\vec{F} \cdot \vec{J}\rangle$ can be rewritten using the following relation

$$
\vec{F}+\vec{J}=\vec{I}+2 \vec{J}
$$

Squaring both sides of this equation and using

$$
\vec{I} \cdot \vec{J}=\frac{1}{2}\left(F^{2}-I^{2}-J^{2}\right)
$$

reveals

$$
\langle\vec{F} \cdot \vec{J}\rangle=\frac{F^{2}+J^{2}-I^{2}}{2} .
$$


Following the same logic leads to a similar equation for the second expectation value

$$
\langle\vec{F} \cdot \vec{I}\rangle=\frac{F^{2}+I^{2}-J^{2}}{2} .
$$

If these terms are collected and evaluated in the coupled basis $\left|I, J, F, F_{z}\right\rangle$ one finds

$g_{F}=g_{J} \frac{F(F+1)-I(I+1)+J(J+1)}{2 F(F+1)}+g_{I} \frac{F(F+1)+I(I+1)-J(J+1)}{2 F(F+1)}$.

This still leaves the problem of determining $g_{J}$ and $g_{I}$. The latter accounts for the complex structure of the nucleus and so its value is found experimentally. The value of $g_{J}$, however, can be found in a manner very similar to that used to find $g_{F}$, the difference being that one must consider the coupling between $\vec{S}$ and $\vec{L}$ and take the projection of these vectors along $\vec{J}$. The result is

$g_{J}=g_{L} \frac{J(J+1)-S(S+1)+L(L+1)}{2 J(J+1)}+g_{S} \frac{J(J+1)+S(S+1)-L(L+1)}{2 J(J+1)}$,

where $g_{S}$ and $g_{L}$ are the electron spin and electron orbital "g-factors."

The value of each of the "g-factors" considered above are listed in Table 2.3. The value of $g_{S}$ is known extraordinarily well and its measurement [52] has served as an important test of QED theory [53]. The value of $g_{L}$ quoted is derived from

$$
g_{L} \approx 1-\frac{m_{e}}{m_{n u c}}
$$


where $m_{n u c}$ is the nuclear mass. This value differs slightly from 1 due to finite nuclear mass and is correct to lowest order in $m_{e} / m_{n u c}$ [54]. The value of $g_{I}$ accounts for the complicated structure of the nucleus and is an experimental value [45]. Finally, the values listed for $g_{J}$ are all experimental values [45], the exception being the value given for the state $5^{2} P_{1 / 2}$ which is calculated from theory.

\begin{tabular}{|c|c|}
\hline$g_{S}$ & $2.0023193043622(15)$ \\
\hline$g_{L}$ & 0.99999369 \\
\hline$g_{I}$ & $-0.0009951414(10)$ \\
\hline$g_{J}\left(5^{2} S_{1 / 2}\right)$ & $2.00233113(20)$ \\
\hline$g_{J}\left(5^{2} P_{1 / 2}\right)$ & $2 / 3$ \\
\hline$g_{J}\left(5^{2} P_{3 / 2}\right)$ & $1.3362(13)$ \\
\hline
\end{tabular}

Table 2.3: ${ }^{87} \mathrm{Rb} D_{2}$ Transition "g-factors"

The expression for $g_{J}$ given in Eq. 2.29 can be simplified by taking the approximate values $g_{S} \approx 2$ and $g_{L} \approx 1$ yielding,

$$
g_{J} \approx \frac{3}{2}+\frac{S(S+1)-L(L+1)}{2 J(J+1)} .
$$

Likewise a simplification the expression for $g_{F}$, given in Eq. 2.28, can be made by neglecting the nuclear term

$$
g_{F} \approx g_{J} \frac{F(F+1)-I(I+1)+J(J+1)}{2 F(F+1)},
$$

yielding an expression which is still correct to the $0.1 \%$ level.

As an example consider the $F=1$ manifold in ground state ${ }^{87} \mathrm{Rb}$ (see Fig. 2.1). In this state $S=1 / 2, L=0, J=1 / 2, I=3 / 2$ and $F=1$ so 
$g_{F}=-1 / 2$ using the approximate expressions for this value and for $g_{J}$ derived above. In the low field limit, the magnetic sublevels will shift in energy in response to an external magnetic field according to

$$
\Delta E_{Z E}=\mu_{B} g_{F} m_{F} B
$$

Recall that because $F=1, m_{F}$ can take on the values of $-1,0$, and 1 . This splitting is shown in Fig. 2.3 and is the basis for magnetically trapping neutral atoms, the topic of the next section. Note that because the value of $g_{F}$ in negative in this state, the sublevel with $m_{F}=-1$ increases in energy with increasing magnetic field while the sublevel with $m_{F}=1$ decreases in energy. The sublevel with $m_{F}=0$ is unaffected to first order by the presence of the external magnetic field.

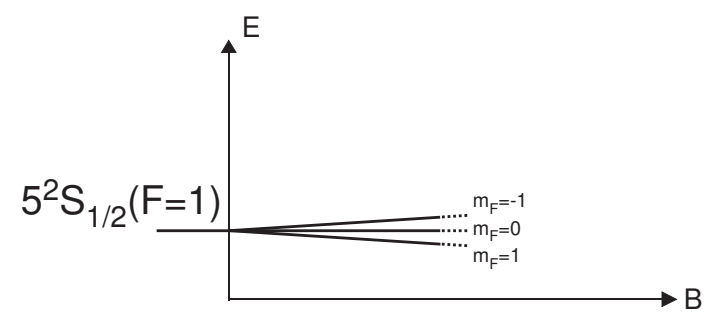

Figure 2.3: Zeeman spitting of the $F=1$ hyperfine manifold. Because $F=1$, $m_{F}$ can take on the values $-1,0$, and 1 . The value of $g_{F}$ in this state is $-1 / 2$ so the magnetic sublevel with $m_{F}=-1$ increases in energy with increasing magnetic field while the sublevel with $m_{F}=1$ decreases in energy. The energy of the $m_{F}=0$ sublevel is unaffected to first order. 


\subsection{Magnetic Trapping}

The Zeeman effect can be used to spatially confine neutral atoms. This feat was first accomplished in 1985 on a sample of neutral atomic sodium [9]. This can be understood by a quite straightforward extension of the main result of the previous section where it was shown that in the low field limit an external magnetic field shifts the magnetic sublevels of an atom by an amount given by

$$
\Delta E_{Z E}=\mu_{B} g_{F} m_{F}|B| .
$$

This shift in energy is correctly viewed as a confining potential for the atoms, the force on which is found from taking the gradient of this expression.

$$
\vec{F}_{Z E}=-\mu_{B} g_{F} m_{F}(\nabla|B|)
$$

In the previous two equations I have been explicit that it is the magnitude of the magnetic field which is important and not the vector quantity. The reason for this is that under certain conditions, which are met in our trap almost everywhere, the atomic magnetic moments follow the direction of the confining magnetic field. The condition for this adiabatic following can be written as $\omega_{L} \gg|d B / d t| / B$, where $\omega_{L}=\mu B / \hbar$ is the Larmor precession rate in the applied magnetic field. If this condition is not met, it can result in trap loss via a process known as Majorana spin flips.

Examination of Eqs. 2.34 and 2.35 reveals that there are two main classes of atomic states with respect to magnetic trapping. The first, commonly called high-field seeking states, satisfy $g_{F} m_{F}<0$ and therefore mini- 
mize their energy in high magnetic fields. The second, called low-field seeking states, satisfy $g_{F} m_{F}>0$ and minimize their energy in magnetic field minima. Earnshaw's theorem prohibits an electrostatic field from stably trapping a charged particle, however it does not rule out the possibility of trapping a dipole [38]. Clearly, for a trap to be stable atoms must accumulate at a field extrema, but local maxima in the absence of sources are forbidden by Maxwell's equations [55]. Therefore atoms must be trapped at a local field minimum, so low-field seeking atomic states must be used.

Ground state ${ }^{87} \mathrm{Rb}$ has three low-field seeking states. They are $|2,2\rangle$, $|2,1\rangle$ and $|1,-1\rangle$, where the notation $\left|F, m_{F}\right\rangle$ is being used. The term "ground state" refers to all of the states contained in the $5^{2} S_{1 / 2}$ spectroscopic term, not just the one with the lowest energy. The state $|2,2\rangle$ couples most strongly to external magnetic fields, providing the tightest confinement for a given magnetic field. For this reason we trap ${ }^{87} \mathrm{Rb}$ atoms in this state.

One may worry that this state is not the lowest in energy and may decay into the $F=1$ manifold, disrupting the experiment. This concern can be put to rest because this is not an allowed electric dipole transition as $\Delta L=0$ and so the lifetime of atoms in the $F=2$ manifold is much longer than the duration of the experiment.

While there are many electric current configurations which can form suitable trapping potentials [56] we use the simplest configuration possible. Our field is produced from a pair of circular coils with counter-propagating currents. This geometry is known as the anti-Helmholtz configuration and it 
produces a quadrupole magnetic field.

The form of the magnetic field produced in this configuration can be arrived at by first considering the form of the magnetic field produced by a single circular loop carrying a current $I$ in a region of permittivity $\mu$ which is given by [56]

$$
\begin{aligned}
& B_{z}=\frac{\mu I}{2 \pi} \frac{1}{\sqrt{(R+\rho)^{2}+(z)^{2}}}\left[K\left(k^{2}\right)+\frac{R^{2}-\rho^{2}-z^{2}}{(R-\rho)^{2}+z^{2}} E\left(k^{2}\right)\right] \\
& B_{\rho}=\frac{\mu I}{2 \pi \rho} \frac{z}{\sqrt{(R+\rho)^{2}+(z)^{2}}}\left[-K\left(k^{2}\right)+\frac{R^{2}+\rho^{2}-z^{2}}{(R-\rho)^{2}+z^{2}} E\left(k^{2}\right)\right]
\end{aligned}
$$

where

$$
k^{2}=\frac{4 R \rho}{(R+\rho)^{2}+z^{2}}
$$

and $K\left(k^{2}\right)$ and $E\left(k^{2}\right)$ are the complete elliptic integrals of the first and second kind, respectively [57]. The terms in this expression are clarified in Fig. 2.4.

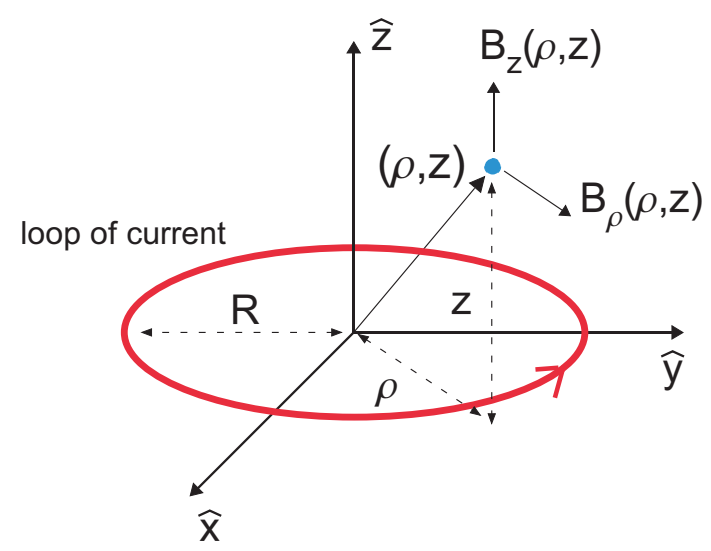

Figure 2.4: Geometry of Eq. 2.36. The red loop of current produces a magnetic field at the point $(\rho, z)$. The magnetic field is decomposed into components parallel to $\hat{z}$ and to $\hat{\rho}$. 
If we now consider the field from two such loops separated by a distance $d$ with equal but opposite current we can arrive at the expression for the magnetic field in the anti-Helmholtz configuration. The full mathematical form is not very illuminating, but a plot of the result is shown in Fig. 2.5. This figure shows the magnitude of the magnetic field along the axis of symmetry of the two loops. The central portion of this potential is used for trapping. Note that near the center the potential is nearly linear, deviating only in the vicinity of the current carrying loops, at the surface of which the field magnitude reaches its maximum value.

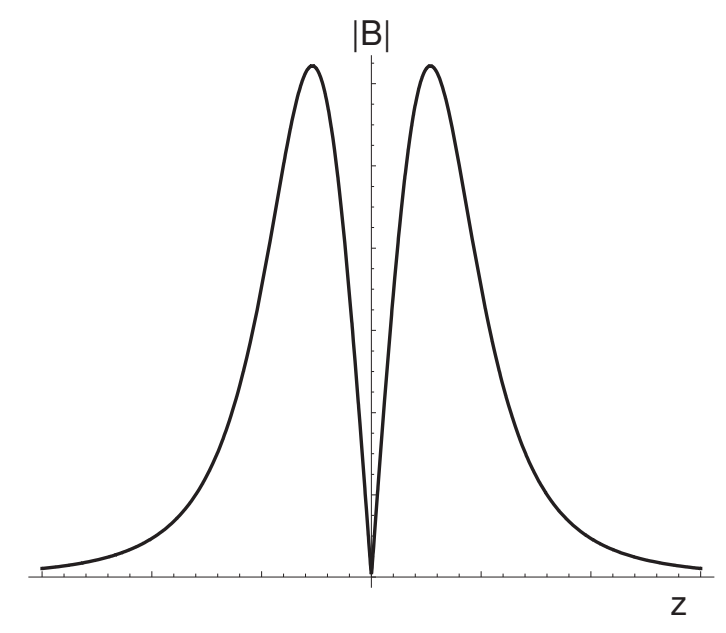

Figure 2.5: Magnitude of the magnetic field along the symmetry axis of the two loops. The central portion of the potential is used for trapping. The potential is approximatly linear near the center and reaches its maximum value at the surface of the circular loops of current.

Luckily, we can justify making a huge simplification because the trapped atoms in this experiment only occupy the region near the center of the trap. 
We can therefore Taylor expand the full solution and take only the leading, linear term. The result of this expansion and simplification is

$$
\begin{aligned}
& B_{z}=2 B^{\prime} z \\
& B_{\rho}=B^{\prime} \rho
\end{aligned}
$$

where the field gradient $B^{\prime}$ is

$$
B^{\prime}=\frac{3}{2} \mu I \frac{(d / 2) R^{2}}{\left[(d / 2)^{2}+R^{2}\right]^{5 / 2}} .
$$

For magnetic trapping the magnitude of the field is the important quantity. It is given by

$$
B=B^{\prime} \sqrt{\rho^{2}+4 z^{2}} .
$$

Note that this potential is linear in all radial directions, however the gradient varies along each direction because of the factor of 4 in Eq. 2.40. Also this potential is not harmonic nor central, and so angular momentum is not conserved in this trap.

The main source of trap loss is collisions between trapped atoms and the residual thermal background gas present in our vacuum chamber. Unfortunately, the cross section for destructive collisions is large because even large impact parameter collisions can impart enough energy to eject atoms from our trap. At pressures $P$, which are low enough to be of practical interest, the trapping time can be approximated by [38]

$$
t \sim\left(10^{-8} / P\right) \mathrm{s}
$$


where $P$ is in torr. The lifetime of atoms in our magnetic trap is $\sim 30 \mathrm{~s}$, suggesting that the background pressure is $\sim 3 \times 10^{-10}$ torr.

\subsection{Interaction of Light with Atoms}

Interactions of light with atoms fall into two very broad regimes. In the first, the so called far-detuned regime, the frequency of light is far from any atomic resonance. The primary effect of the light is to mix states of opposite parity, inducing a dipole moment in the atom [58]. The induced dipole moment can then interact with an optical intensity gradient present in the dipole inducing beam. As will be discussed, this situation can lead to an almost completely conservative optical trap for neutral atoms. In the second, near-resonant regime, light interacts with atoms primarily through forces due to photon scattering. As will be explored in some detail, this regime can be used to cool and trap atomic ensembles.

\subsubsection{The Optical Dipole Force}

The use of the optical dipole force for confining atoms was first considered by Askar'yan in 1962 [59]. Eight years later Ashkin trapped micron-sized particles with a laser using a combination of radiation pressure and the optical dipole force [60]. He later suggested a 3-D trap for neutral atoms based on his previous work [61]. In 1978 Bjorkholm experimentally demonstrated the dipole force by focusing a beam of neutral atoms [62]. These ideas and work culminated in 1986 with the first optical dipole trap for neutral atoms 
demonstrated by Chu et al. [63].

To model the dipole force, let the applied electric field and induced dipole moment be given as

$$
\begin{aligned}
\vec{E}(\vec{r}, t) & =\hat{e} \tilde{E}(\vec{r}) e^{-i \omega t}+\text { c.c. } \\
\vec{p}(\vec{r}, t) & =\hat{e} \tilde{p}(\vec{r}) e^{-i \omega t}+\text { c.c. }
\end{aligned}
$$

where these two quantities are related through the complex polarizability $\alpha$

$$
\vec{p}=\alpha(\omega) \vec{E}
$$

The potential energy of the induced dipole in the driving electric field is

$$
U_{d i p}=-\frac{1}{2}\langle\vec{p} \cdot \vec{E}\rangle
$$

where the angular brackets represents a time average and the $1 / 2$ accounts for the induced, not permanent, nature of the electric dipole. This can be written in a more useful form by replacing the amplitude of the electric field with the corresponding intensity through $I=2 \epsilon_{0} c|\tilde{E}|^{2}$ and evaluating the time average of the two complex quantities,

$$
U_{\text {dip }}=-\frac{1}{2 \epsilon_{0} c} \operatorname{Re}(\alpha) I
$$

We see that the potential is proportional to the intensity of light and the real part of the polarizability, the latter of which represents the in-phase 
component of the dipole oscillation. The dipole force can be found from the gradient of the potential energy and is therefore a conservative field.

$$
\vec{F}_{d i p}=-\nabla U_{d i p}(\vec{r})=\frac{1}{2 \epsilon_{0} c} R e(\alpha) \nabla I(\vec{r})
$$

Thus the optical dipole force comes from the dispersive interaction of the induced dipole moment with the gradient of the driving light field [59, 64-66].

We now turn our attention to modeling $\alpha$ which we do following Lorentz's model of a classical oscillator. In this model the electron is considered to be a classical particle bound elastically to a nucleus and possessing an oscillation frequency $\omega_{0}$ which is identified with the frequency of the optical transition of interest. Damping is included in this model through Larmor's formula for the power radiated by an accelerating charge [67]. This model has several limitations - it does not model an atom with multiple transitions and does not display any saturation behavior when strongly driven. In the far-detuned limit neither of these limitations are of concern.

The equation of motion for a damped, driven harmonic oscillator

$$
\ddot{x}+\Gamma_{L} \dot{x}+\omega_{0}^{2} x=-\frac{e E(t)}{m_{e}}
$$

can be used to solve for $\alpha$ by noting that $p=-e x=\alpha E$. The result is

$$
\alpha=\frac{e^{2}}{m_{e}} \frac{1}{\omega_{0}^{2}-\omega^{2}-i \omega \Gamma_{L}}
$$

where the damping coefficient $\Gamma_{L}$ is the classical Larmor energy damping rate due to radiative loss

$$
\Gamma_{L}=\frac{e^{2} \omega^{2}}{6 \pi \epsilon_{0} m_{e} c^{3}}
$$


Using this relation and introducing the on-resonance damping rate $\Gamma \equiv \Gamma_{\omega_{0}}=$ $\left(\omega_{0} / \omega\right)^{2} \Gamma_{L}$ allows the polarizability to be written as [68]

$$
\alpha=6 \pi \epsilon_{0} c^{3} \frac{\Gamma / \omega_{0}^{2}}{\omega_{0}^{2}-\omega^{2}-i\left(\omega^{3} / \omega_{0}^{3}\right) \Gamma} .
$$

Interestingly, the semi-classical approach which treats the atom as a two-level quantum system interacting with a classical field yields the same result in the low-saturation limit with one notable exception. The damping rate must be found from the dipole matrix element between the ground and excited state.

$$
\Gamma=\frac{\omega_{0}^{3}}{3 \pi \epsilon_{0} \hbar c^{3}}|\langle e|\mu| g\rangle|^{2}
$$

But for the D lines in alkali atoms, including ${ }^{87} \mathrm{Rb}$, which are strongly allowed dipole transitions the classical estimation agrees with the true decay rate to within a few percent [68].

Using the classical estimation for $\alpha$ in the equation for the dipole potential (Eq. 2.46) yields a very useful result, valid in the limit of large detuning and negligible saturation

$$
U_{d i p}(\vec{r})=\frac{3 \pi c^{2}}{2 \omega_{0}^{3}} \frac{\Gamma}{\Delta} I(\vec{r})
$$

Here $\Delta \equiv \omega-\omega_{0}$ is the optical detuning from resonance. $U_{d i p}$ is inversely proportional to the optical detuning which can take on both positive and negative values. When $\omega>\omega_{0}$, so-called blue-detuning, the potential is positive, and atoms experience a repulsive force pushing them away from local intensity maxima. When $\omega<\omega_{0}$, so-called red-detuning, atoms are pulled into local 
maxima. Our experiment makes extensive use of blue-detuned optical sheets which act as repulsive walls. We combine several of these sheets to form optical boxes for trapping atoms. Specific detail on this technique can be found in Ch. 4.

Although not derived here, a very similar consideration leads to an expression for the optical scattering rate valid in the same limits as Eq. 2.53 [68].

$$
\Gamma_{s c}(\vec{r})=\frac{3 \pi c^{2}}{2 \hbar \omega_{0}^{2}}\left(\frac{\Gamma}{\Delta}\right)^{2} I(\vec{r})
$$

Notice that the scattering rate varies as $\sim 1 / \Delta^{2}$, so that for a sufficiently large detuning the optical dipole force completely dominates forces due to optical scattering.

\subsubsection{Scattering Forces}

When an optical field is near an atomic resonance it is scattering forces that dominate the atom-light interaction. The non-conservative nature of the spontaneous scattering process means that radiative forces can be used to cool atomic ensembles. Here I will discuss the scattering rate and resulting force in the semi-classical approximation, treating the atom as a two-level quantum system and the optical field as classical. I will then shows how this is used to cool and confine atoms in our experiment. 


\subsubsection{Optical Bloch Equations}

The optical Bloch equations are used to describe an ensemble of twolevel atoms interacting with a monochromatic optical field [69] as shown in Fig. 2.6. To describe the situation in the figure we must find the expectation

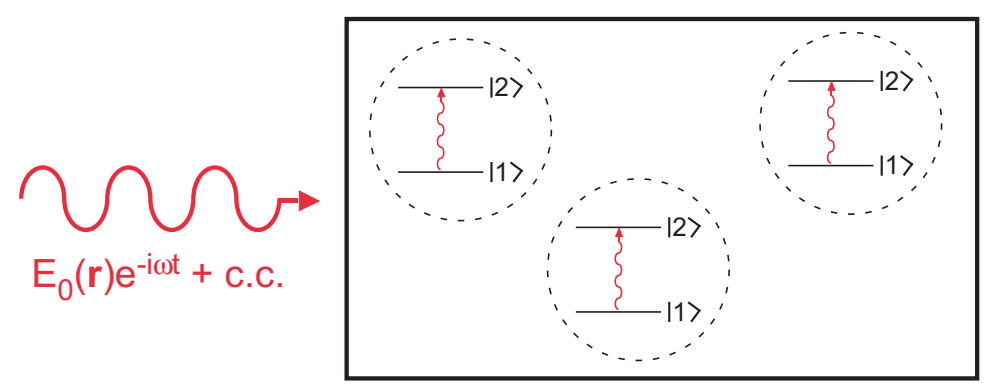

Figure 2.6: Ensemble of $\mathrm{N}$ two-level atoms per unit volume interacting with a monochromatic optical field.

value

$$
\langle\vec{\mu}(t)\rangle=\int \Psi^{*} \vec{\mu} \Psi d^{3} r
$$

of the microscopic polarization operator $\vec{\mu}=-e \vec{r}$ by solving Schrödinger's equation,

$$
i \hbar \frac{\partial \Psi}{\partial t}=H \Psi
$$

Here $H=H_{0}+H_{c o h}+H_{d a m p}$ is the sum of the unperturbed atomic Hamiltonian $H_{0}$, the Hamiltonian describing the coherent evolution of the atom driven by the optical field $H_{c o h}=-\vec{\mu} \cdot \vec{E}(t)$ and the Hamiltonian due to inter-atomic damping processes such as collisions. The inclusion of the third term means that we must consider the system as a quantum ensemble, not as individual atoms. Because the system we are describing comprises a large number of 
atoms its wavefunction $\Psi(t)$ is typically intractable. Instead we use the density matrix formalism which provides a convenient way of describing the evolution of the ensemble average expectation value of the dipole operator $\overline{\langle\mu(t)\rangle}$ even when $\Psi(t)$ is unknown.

The density matrix can be defined in two steps. In the first step, we consider the expectation value of the dipole operator for a single atom

$$
\langle\mu(t)\rangle=\langle\Psi(\vec{r}, t)|\mu| \Psi(\vec{r}, t)\rangle .
$$

We then expand $\Psi(\vec{r}, t)$ in a complete set of orthonormal eigenfunctions of the unperturbed atomic Hamiltonian $H_{0}$

$$
\Psi(\vec{r}, t)=\sum_{n} c_{n}(t) u_{n}(\vec{r})
$$

where all of the time dependence has been placed in the coefficients $c_{n}(t)$. We then evaluate the expectation value of the dipole operator in this basis

$$
\langle\mu(t)\rangle=\sum_{m, n} c_{m}^{*}(t)\left\langle u_{m}(\vec{r})|\mu| u_{n}(\vec{r})\right\rangle c_{n}(t)
$$

By defining $\mu_{m n} \equiv\left\langle u_{m}(\vec{r})|\mu| u_{n}(\vec{r})\right\rangle$ and $R_{n m} \equiv c_{n} c_{m}^{*}$ this can be written much more compactly as

$$
\langle\mu(t)\rangle=\sum_{m, n} R_{n m} \mu_{m n}=\sum_{n}(R \mu)_{n n}=\operatorname{Tr}(R \mu) .
$$

Next we apply this approach to an atomic ensemble. To do so we define

$$
\rho_{n m}(t) \equiv \overline{R_{n m}}(t)
$$


so that the ensemble average expectation value of the dipole operator can be expressed as

$$
\overline{\langle\mu(t)\rangle}=\sum_{m, n} \overline{c_{n} c_{m}^{*}} \mu_{m n}=\operatorname{Tr}[\rho(t) \mu]
$$

The density matrix has four elements in this case because we have assumed a two-level system,

$$
\rho=\left(\begin{array}{cc}
\rho_{11} & \rho_{12} \\
\rho_{21} & \rho_{22}
\end{array}\right)
$$

The diagonal elements represent the probability of finding an atom in the respective state i.e. $\rho_{11}$ is the probability of finding an atom in state $|1\rangle$. The significance of the off-diagonal elements can be seen by calculating $\overline{\langle\mu(t)\rangle}$ for our two level system. The result is

$$
\overline{\langle\mu(t)\rangle}=\rho_{12} \mu_{21}+\rho_{11} \mu_{11}+\rho_{22} \mu_{22}+\rho_{21} \mu_{12} .
$$

If we assume that each state $|1\rangle$ and $|2\rangle$ has definite parity and note that the dipole operator $\mu$ has odd parity symmetry then we see immediately that $\mu_{11}=\mu_{22}=0$. Additionally if we assume, with no loss of generality, that $\mu_{21}=\mu_{12} \equiv \mu$ then we can write the very illuminating formula

$$
\overline{\langle\mu(t)\rangle}=\mu\left[\rho_{12}(t)+\rho_{21}(t)\right]
$$

which shows that the sum of off-diagonal elements, known as the coherence terms, is proportional to the ensemble average dipole moment.

We are now in a position to derive the optical Bloch equations. The temporal evolution of the operator $\rho$ is determined by the Heisenberg equation

$$
\dot{\rho}=\frac{1}{i \hbar}[H, \rho] \text {. }
$$


In this equation we let $H=H_{0}+H_{\text {coh }}$ and neglect $H_{\text {damp }}$ because its exact form is unknown and its effect can be added in later by hand. With this approximation we find the time evolution of the off-diagonal elements to be

$$
\dot{\rho}_{21}=-i \omega_{0} \rho_{21}+i \frac{\mu E(t)}{\hbar}\left(\rho_{11}-\rho_{22}\right)
$$

with $\dot{\rho_{12}}=\dot{\rho_{21}}{ }^{*}$. The diagonal elements are found to be

$$
\dot{\rho}_{11}=-\frac{\mu E(t)}{i \hbar}\left(\rho_{21}-\rho_{12}\right)=-\dot{\rho}_{22} .
$$

Damping is a property of the interaction between individual dipoles and is therefore a property of the ensemble and not individual atoms. Damping can be included phenomenologically in the off-diagonal elements from the knowledge that when $E(t) \rightarrow 0$ the ensemble average dipole moment must decay to 0 . There are many causes of dephasing which lead to the decay of the off-diagonal element such as population relaxation, collisions, and dipoledipole interactions, the net effect of which can be encompassed in a transverse decay rate $1 / T_{2}$. Likewise, processes such as spontaneous decay and collisional de-excitation lead to the decay (growth) of the excited (ground) state population. The rate of these processes can be encompassed by the longitudinal decay rate $1 / T_{1}$.

Before including the effects of damping in the optical Bloch equations, I would like to briefly discuss the commonly used rotating wave approximation, which is a result of writing the off-diagonal elements as a slowly varying envelope function multiplied by a response at the applied optical frequency.

$$
\rho_{21}(t)=\sigma_{21}(t) e^{-i \omega t}=\rho_{12}^{*}(t)
$$


When these expressions are substituted into the optical Bloch equations and terms proportional to $e^{-i \omega t}$ are equated while terms proportional to $e^{-2 i \omega t}$ are discarded, the equations take on their familiar form.

$$
\begin{aligned}
& \dot{\rho}_{11}=\frac{i \Omega}{2}\left(\sigma_{12}-\sigma_{21}\right)+\frac{\rho_{22}}{T_{1}} \\
& \dot{\rho}_{22}=-\frac{i \Omega}{2}\left(\sigma_{12}-\sigma_{21}\right)-\frac{\rho_{22}}{T_{1}} \\
& \dot{\sigma}_{12}=-\left(\frac{1}{T_{2}}+i \Delta\right) \sigma_{12}-\frac{i \Omega}{2}\left(\rho_{22}-\rho_{11}\right)
\end{aligned}
$$

The off-diagonal terms are complex conjugates of each other $\dot{\sigma}_{21}=\dot{\sigma}_{12}^{*}$, the detuning of the applied field from resonance is denoted $\Delta \equiv \omega-\omega_{0}$, and $\Omega=-\vec{\mu} \cdot \vec{E}_{0} / \hbar$ is the Rabi frequency.

If we assume that radiative decay is the only dephasing process then $1 / T_{1}=\Gamma$ and $1 / T_{2}=\Gamma / 2$, where $\Gamma$ is the natural decay rate of the excited state. With this assumption, the steady state solution of the excited population is

$$
\rho_{22}=\frac{(\Omega / \Gamma)^{2}}{1+4(\Delta / \Gamma)^{2}+2(\Omega / \Gamma)^{2}} .
$$

This can be put in a more useful form for experimentalists by introducing the transition saturation intensity $I_{\text {sat }}$

$$
\frac{I}{I_{\text {sat }}}=2 \frac{\Omega^{2}}{\Gamma^{2}}
$$

so that we may write

$$
\rho_{22}=\frac{1}{2} \frac{\left(I / I_{\text {sat }}\right)}{1+4(\Delta / \Gamma)^{2}+\left(I / I_{\text {sat }}\right)}
$$


The significance of $I_{\text {sat }}$ is that at saturation the Rabi frequency has a value comparable to the natural decay rate $\Gamma$ and that $\rho_{22}$ reaches $1 / 2$ of its maximum value. With the substitution $I=(1 / 2) c \epsilon_{0} E_{0}^{2}$, the saturation intensity can be written

$$
I_{s a t}=\frac{\epsilon_{0} c \Gamma^{2} \hbar^{2}}{4|\hat{e} \cdot \vec{\mu}|^{2}}
$$

where $\hat{e}$ is the direction of the polarization of the electric field. The value of the saturation intensity depends on the transition strength through $\Gamma$ as well as the relative orientation of the polarization of light and transition dipole moment, but for simplicity the minimum value is usually reported as a representative figure. For the $D_{2}\left(F=2 \rightarrow F^{\prime}=3\right)$ transition $I_{\text {sat }}=3.57713(74) \mathrm{mW} / \mathrm{cm}^{2}$ for light with isotropic polarization [43].

A consequence of saturation is that the measured width of a transition increases with increasing probe intensity due to a phenomena known as saturation broadening. The measured full width at half maximum (FWHM) increases with intensity as [70]

$$
\Delta \omega_{F W H M}=\Gamma\left(1+\frac{I}{I_{\text {sat }}}\right)^{1 / 2} .
$$

\subsubsection{Optical Molasses}

Optical Molasses is a laser cooling technique commonly used to reduce the temperature of an atomic vapor. Because atoms in a gas move in all directions the applied laser beams must cool in all three dimensions by interacting

with the gas along three orthogonal dimensions as shown in Fig. 2.7. In this 


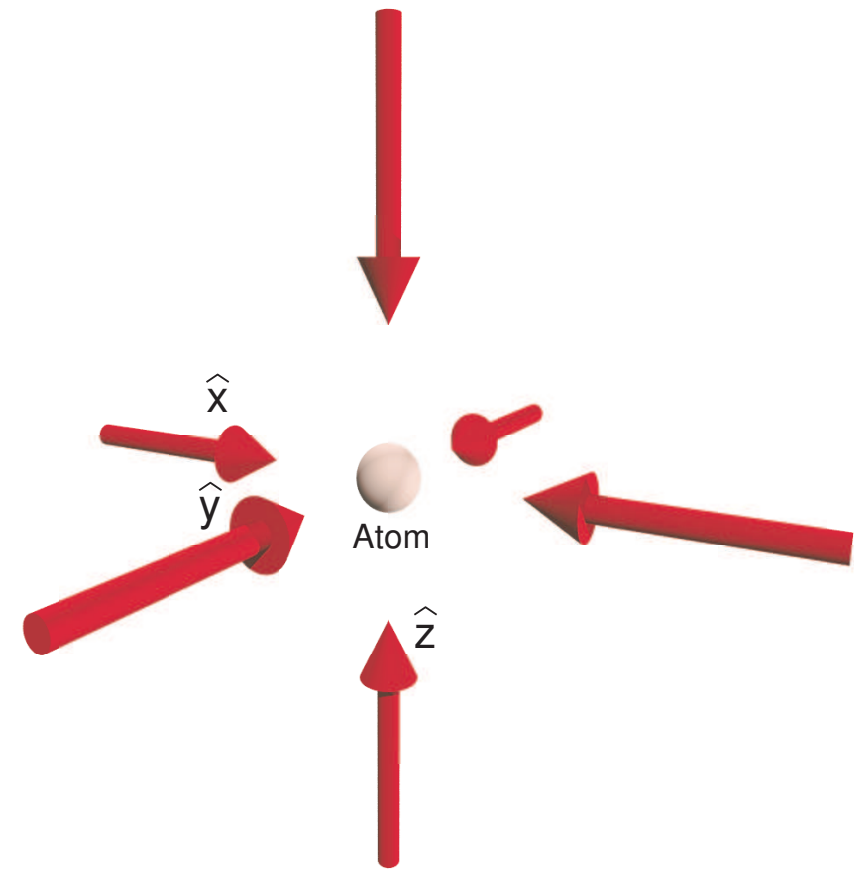

Figure 2.7: Three pairs of counter propagating laser beams impinge on an atom. Each beam is detuned below the atomic transition resonance by the same amount.

figure three pairs of counter propagating beams, all at the same frequency and intensity, interact with an atom. It might appear that this arrangement will have no effect on the atom because the effect from counter propagating beams will cancel. This is true for a stationary atom, but for an atom in motion the symmetry of the arrangement is broken. The Doppler effect causes the atom to scatter photons out of counter propagating beams at different rates, leading to an imbalance of the force on the atom. Consider the 1-D situation depicted in Fig. 2.8 in which each of the counter propagating beams is tuned below the atomic resonance frequency $\omega_{0}$ by the same amount. If the atom moves 

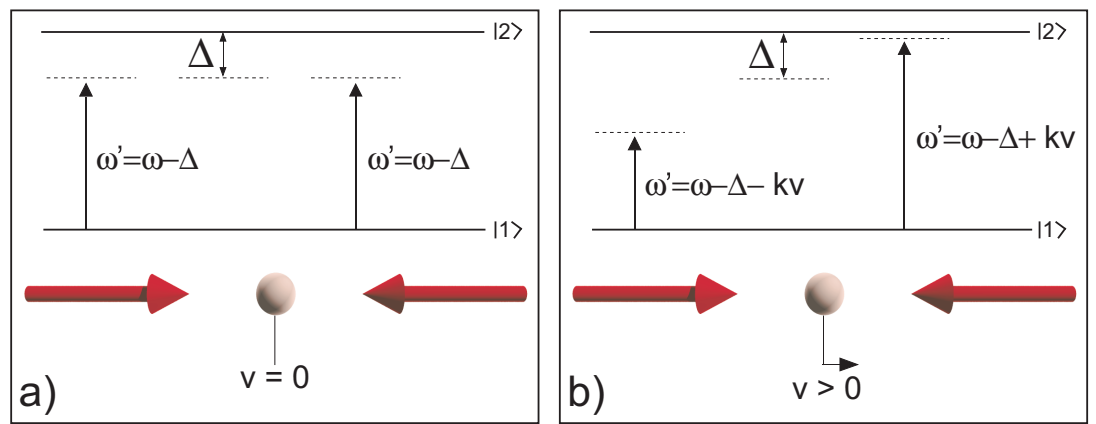

Figure 2.8: 1-D optical molasses. A pair of counter propagating beams detuned below the atomic resonance frequency impinge on an atom. (a) If the atom is at rest then it sees each beam with equal detuning from resonance $\Delta$ and scatters photons out of each beam equally. (b) If the atom is in motion the Doppler effect shift the beam opposing the atomic motion into resonance and the atom scatters photon preferentially from this beam, slowing it.

to the right, the Doppler effect will cause the atom to scatter photons from the leftward traveling beam at a rate greater than from the rightward traveling beam. Of course the opposite effect will take place if the atoms moves to the left. This leads to a force which tends to slow the atom regardless of its direction of travel.

This argument can be made somewhat more quantitative by using a result from the previous section where we calculated the excited state occupation probability for a two level atom interacting with a monochromatic beam

$$
\rho_{22}=\frac{1}{2} \frac{\left(I / I_{\text {sat }}\right)}{1+4(\Delta / \Gamma)^{2}+\left(I / I_{\text {sat }}\right)}
$$

The total scattering rate $R_{\text {scatt }}=\Gamma \rho_{22}$ for a single atom is the product of the 
excited state occupation probability and the natural decay rate

$$
R_{\text {scatt }}=\frac{\Gamma}{2} \frac{\left(I / I_{\text {sat }}\right)}{1+4(\Delta / \Gamma)^{2}+\left(I / I_{\text {sat }}\right)} .
$$

Since each photon scattered carries momentum $\hbar k$ the force on the atom due to this process is

$$
F_{\text {scatt }}=\hbar k \frac{\Gamma}{2} \frac{\left(I / I_{\text {sat }}\right)}{1+4(\Delta / \Gamma)^{2}+\left(I / I_{\text {sat }}\right)} .
$$

We can use this result and the first order Doppler shift $\omega^{\prime}=\omega-k v$, where $\omega^{\prime}$ is the frequency seen by an atom moving at velocity $v$ with respect to a laser beam at frequency $\omega$, to find the force on the atom in this arrangement. If we consider the 1-D case, with a single pair of counter propagating beams, then the total force is just the sum of forces due to each beam

$$
F_{\text {molasses }}=F_{\text {scatt }}\left(\omega-\omega_{0}-k v\right)-F_{\text {scatt }}\left(\omega-\omega_{0}+k v\right) .
$$

We can approximate this result for small velocities through Taylor expansion of $F_{\text {scatt }}$

$$
F_{\text {molasses }} \approx F_{\text {scatt }}\left(\omega-\omega_{0}\right)-k v \frac{\partial F}{\partial \omega}-\left[F_{\text {scatt }}\left(\omega-\omega_{0}+k v\right)\right] \approx-2 \frac{\partial F}{\partial \omega} k v .
$$

We note that this may be written

$$
F_{\text {molasses }}=-\alpha v
$$

showing that this force mimics classical viscous damping, the reason it is called optical molasses. The value of $\alpha$ is found through differentiation of Eq. 2.77 to be

$$
\alpha=4 \hbar k^{2} \frac{I}{I_{\text {sat }}} \frac{-2 \Delta / \Gamma}{\left[1+(2 \Delta / \Gamma)^{2}\right]^{2}},
$$


where $I / I_{\text {sat }} \ll 1$ has been assumed. Clearly for damping to occur $\alpha>0$ so the detuning $\Delta=\omega-\omega_{0}<0$ is negative, in agreement with the qualitative picture presented earlier.

There are two features of optical molasses that warrant explicit statements. First, optical molasses only confines atoms in velocity and not configuration space. In other words, it is a cooling and not a trapping technique. Second, even though Eq. 2.80 suggests that after sufficient time $v \rightarrow 0$ hence $T \rightarrow 0$ this is clearly not a physical result. This discussion ignored heating effects due to the random nature of the absorption and spontaneous emission process. The heating and cooling effects of optical molasses reach a balance at the Doppler temperature [70]

$$
T_{D}=\frac{\hbar \Gamma}{2 k_{B}}
$$

This temperature is the lowest value that one expects to cool a two-level system. However, due to magnetic substructure the temperature achieved in experiments are much lower. The effect responsible for this is the topic of the next section.

\subsubsection{Sisyphus Cooling}

As discussed in Sec. 1.1, many groups, most notably the group of W. Phillips, measured temperatures of atomic clouds cooled by optical molasses to be well below the Doppler limit given in Eq. 2.82. This cannot be understood in terms of the simple two-level system used in that discussion. Jean Dalibard 
and Claude Cohen-Tannoudji discovered that cooling beyond the Doppler limit was due to an interaction between the optical field and the atom's magnetic sublevels [12].

The effect responsible for the additional cooling can be understood by considering an atom with a ground state total electronic angular momentum $J=1 / 2$ and an excited state total electronic angular momentum $J^{\prime}=3 / 2$ moving through a standing wave formed by two counter-propagating beams with orthogonal linear polarization.

As indicated in Fig. 2.9(a) the relative strength of the transitions depend on the value of $m_{J}$ in the lower $(J=1 / 2)$ and upper $\left(J^{\prime}=3 / 2\right)$ state. For example, for $\sigma^{+}$polarized light, which by definition drives transitions with $\Delta m_{J}=+1$, the coupling of the $\left|J=1 / 2, m_{J}=1 / 2\right\rangle \rightarrow\left|J=3 / 2, m_{J}=3 / 2\right\rangle$ transition is three times stronger than the coupling of the $\mid J=1 / 2, m_{J}=$ $-1 / 2\rangle \rightarrow\left|J=3 / 2, m_{J}=1 / 2\right\rangle$ transition. On the contrary, for $\sigma^{-}$polarized light, which drives transitions with $\Delta m_{J}=-1$, the coupling of the $\mid J=$ $\left.1 / 2, m_{J}=-1 / 2\right\rangle \rightarrow\left|J=3 / 2, m_{J}=-3 / 2\right\rangle$ transition is three times stronger than the coupling of the $\left|J=1 / 2, m_{J}=1 / 2\right\rangle \rightarrow\left|J=3 / 2, m_{J}=-1 / 2\right\rangle$ transition. The relative strength of all allowed electric dipole transition are given as integer values in this figure and can be calculated using the formalism outlined in Sec. 2.6. 
a)

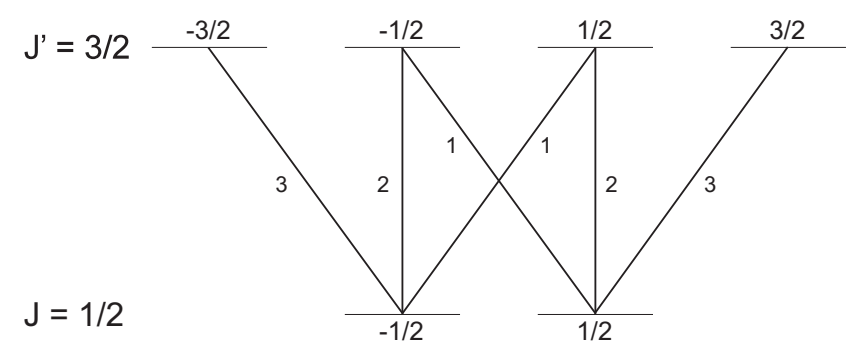

b)
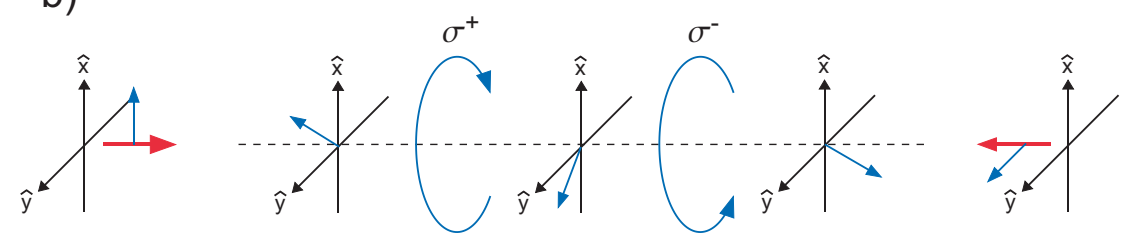

c)

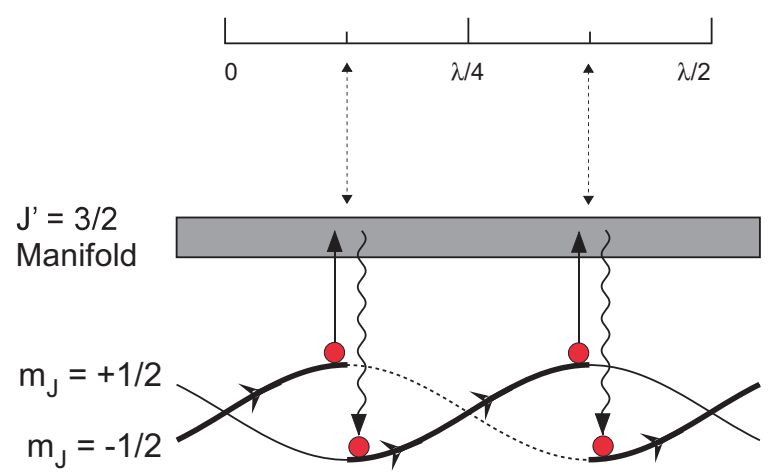

Figure 2.9: The Sisyphus cooling effect. (a) The relative coupling strength of all allowed electric dipole transitions are shown as integer values. (b) The standing wave formed by two counter-propagating beams with orthogonal linear polarizations results in a polarization gradient. (c) The ground state energy levels are shifted periodically by the polarization gradient.

The result of the superposition of the counter-propagating beams with orthogonal linear polarization is a standing wave with a spatially varying polarization. This is indicated in Fig. 2.9(b) where a beam traveling to the 
right and polarized along $\hat{x}$ superposes with a beam traveling in the opposite direction polarized along $\hat{y}$. The local polarization depends on the relative phase difference between the two beams and varies from $\sigma^{+}$to $\sigma^{-}$in a distance $\Delta z=\lambda / 4$. This polarization gradient causes a spatial, periodic modulation of the energy of each of the ground states $\left|J=1 / 2, m_{J}=1 / 2\right\rangle$ and $\left|J=1 / 2, m_{J}=-1 / 2\right\rangle$ of different magnitude given roughly by Eq. 2.53. For example, consider a location at which the local polarization is $\sigma^{+}$. If the laser beams are tuned below the atomic resonance frequency then both ground states will be shifted downwards in energy, however because the coupling between the $\left|J=1 / 2, m_{J}=1 / 2\right\rangle$ and $\left|J=3 / 2, m_{J}=3 / 2\right\rangle$ state is stronger, this ground state is shifted downward more. Conversely, at locations where the light has $\sigma^{-}$polarization the ground state $\left|J=1 / 2, m_{J}=-1 / 2\right\rangle$ is shifted downward in energy more. These two states oscillate in energy along the polarization gradient as shown in Fig. 2.9(c).

If these energy level shifts (known as light shifts) were the only effect the light had on the atoms then we would expect the atoms to exchange potential and kinetic energy as they traveled over the potential hills, but we would not expect any further cooling to take place due to the light. However the light does effect the atoms in another way, it optically pumps them in a spatially dependent way. For example, consider what happens to an atom initially in the $\left|J=1 / 2, m_{J}=-1 / 2\right\rangle$ state located at a position with $\sigma^{+}$light polarization. After being excited into the $\left|J=3 / 2, m_{J}=1 / 2\right\rangle$ state it has two modes of decay. If it decays back into the $\left|J=1 / 2, m_{J}=-1 / 2\right\rangle$ state the 
process repeats. If however, it decays into the $\left|J=1 / 2, m_{J}=1 / 2\right\rangle$ state then it is trapped in a cycling transition and has no way of returning to the $\mid J=$ $\left.1 / 2, m_{J}=-1 / 2\right\rangle$ state. Note that the atom initially in the $\mid J=1 / 2, m_{J}=$ $-1 / 2\rangle$ state climbed a potential hill to reach the region of $\sigma^{+}$polarization where it was optically pumped into the $\left|J=1 / 2, m_{J}=1 / 2\right\rangle$ state which has lower energy due to a larger downward light shift. The atom therefore lost the energy it used to climb the potential hill when it was transfered into the other ground state. This excess energy is carried away by the spontaneously emitted photon which has a higher frequency than the photon which excited the atom into the $J^{\prime}=3 / 2$ manifold. After decay, the atom finds itself at the bottom of a potential valley in the $\left|J=1 / 2, m_{J}=1 / 2\right\rangle$ state. If it climbs to the top of this potential valley it will be in a region of $\sigma^{-}$polarization and will be pumped into the $\left|J=1 / 2, m_{J}=-1 / 2\right\rangle$ state losing more energy in the process. This repeated cycle of climbing a potential hill only to be pumped into a valley is called the 'Sisyphus' effect after a character in Greek mythology condemned by the gods to a similar fate.

This simple picture suggests that this cooling mechanism works until the atoms no longer have sufficient energy to climb the potential hills and are stuck in the valleys. A final temperature can be estimated with this picture in mind [70]

$$
k_{B} T \approx U_{d i p} \propto \frac{I}{|\Delta|} .
$$

where $U_{d i p}$ can be estimated from Eq. 2.53. Of course this technique also has a limit and cannot be used to cool atoms to $0 \mathrm{~K}$ as $\Delta \rightarrow 0$. When the 
energy increase due to the recoil from the spontaneous decay process equals the energy removed from the Sisyphus effect then equilibrium is reached, this occurs at the recoil temperature.

$$
T_{r}=\frac{\hbar^{2} k^{2}}{m k_{B}}
$$

The Sisyphus effect typically results in atomic samples being cooled to a few times the recoil temperature, which for ${ }^{87} \mathrm{Rb}$ is $362.96 \mathrm{nK}$.

\subsubsection{Magneto-Optical Trap}

As discussed in Sec. 2.5.2.2, spontaneous light forces can be used to confine atoms in velocity space if beams are arranged appropriately and tuned slightly below the atomic resonance frequency. Unfortunately, optical molasses does not confine atoms spatially. However, with the correct choice of beam polarizations and the addition of a magnetic field gradient, a hybrid magneto-optical trap (MOT) can be formed which both cools and confines atomic samples. The idea of this extremely useful trap was proposed in 1986 [71] and demonstrated the following year [10]. Since then MOTs have become the most widely used cold atom trap. In fact, our experiment uses two separate MOTs to operate, as discussed in Ch. 3.

Figure 2.10 shows the typical geometry of a MOT. Just like the arrangement in optical molasses, the MOT uses three pairs of counter-propagating beams tuned slightly below the atomic resonance frequency. In this case however, the beams must be chosen to have opposite circular polarization for 

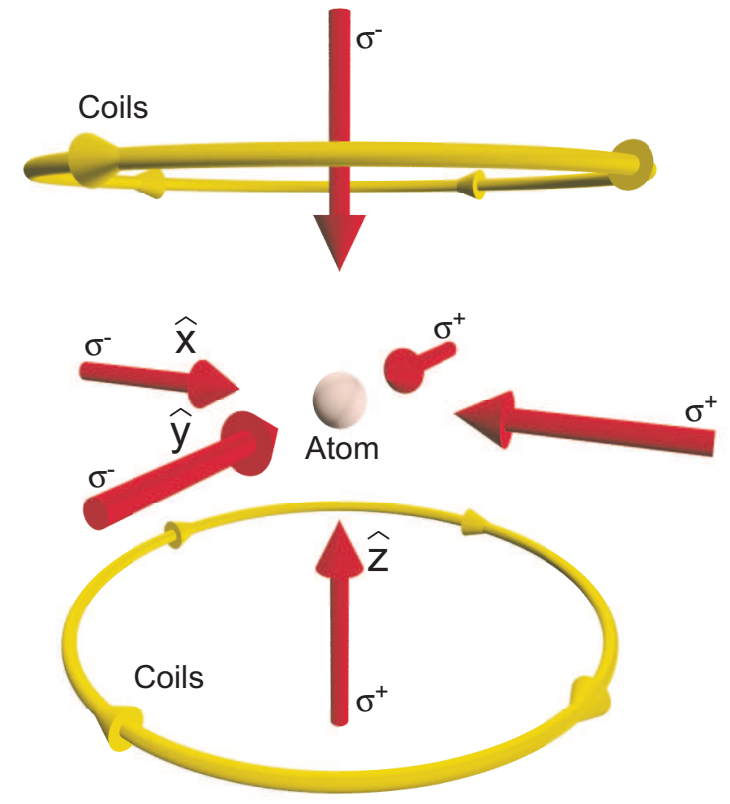

Figure 2.10: Geometry of the magneto-optical trap (MOT). Three orthogonal pairs of counter-propagating beams overlap in the region of a magnetic field gradient. The beams in each pair have opposite circular polarization. The magnetic gradient is produced by two coils (shown in yellow) in the antiHelmholtz configuration.

reasons which will become clear shortly. Additionally, a magnetic field gradient must be introduced into the system, in Fig. 2.10 and our experiment this is done with two coils in the anti-Helmholtz configuration, illustrated as two yellow tori. As discussed in Sec. 2.4 this coil geometry produces a quadrupole field with a linear gradient near the magnetic field center. In contrast to pure magnetic trapping, the magnetic field gradients used in MOTs are often too small to confine atoms-instead the gradient causes an imbalance in the scattering rate for atoms displaced from the magnetic field center and this scattering 
force confines the atoms.

The principle of the MOT is illustrated in Fig. 2.11 for the simple case of an atom with a ground state with $J=0$ and an excited state with $J=1$ in a $1-D$ geometry. Due to the symmetry of the coils the magnetic field

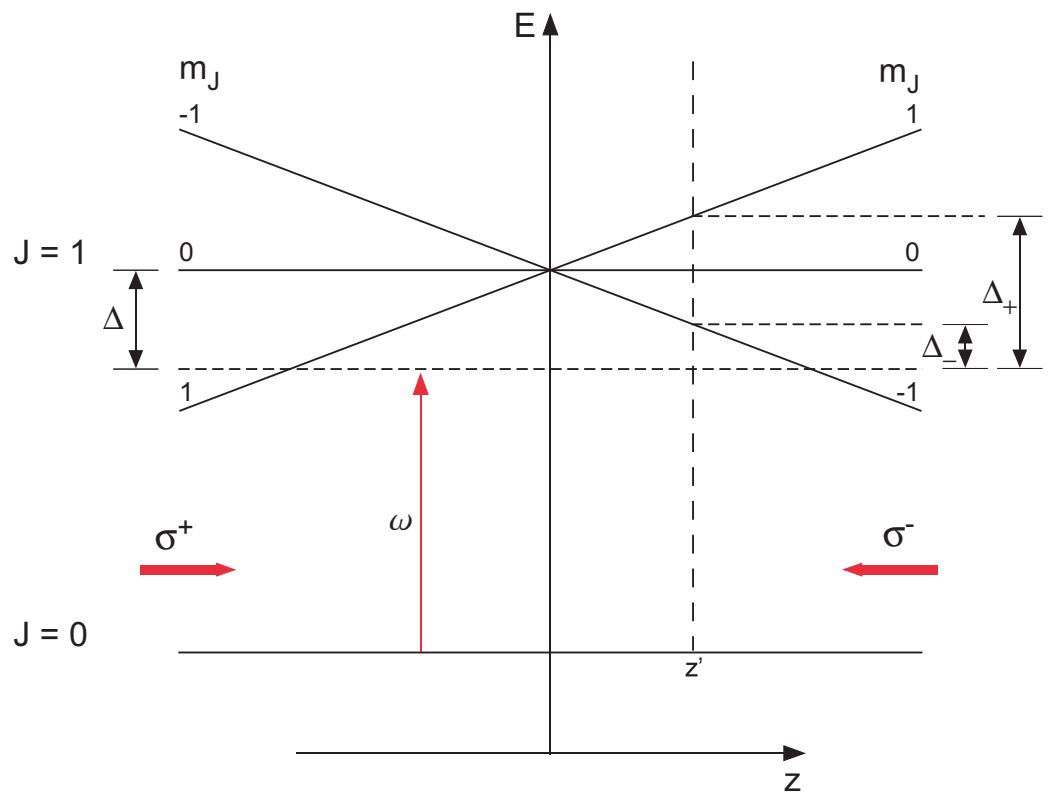

Figure 2.11: A 1-D magneto-optical trap for the simple case of a $J=0 \rightarrow$ $J=1$ transition. A linear magnetic gradient shifts the energy of the $J=1$ Zeeman sublevels linearly with position along $\hat{z}$. A pair of counter-propagating beams with opposite circular polarization tuned below the atomic resonance frequency impinge on the atom. An atom located at $z=z^{\prime}$ has its Zeeman sublevels shifted such that the $m_{J}=-1$ sublevel is closer to atomic resonance than the $m_{J}=1$ sublevel. This causes the atom to scatter photons out of the $\sigma^{-}$beam at a greater rate than out of the oppositely traveling beam. The net force pushes the atom back towards the center of the trap.

vanishes at the center of the pair. Near the field zero the field increases linearly. The magnetic field perturbs the energy of the Zeeman sublevels causing them 
to vary linearly with position along $\hat{z}$. The counter-propagating beams are tuned below the atomic resonance by an amount $\Delta$ and have opposite circular polarizations as shown in the figure. To see how this leads to a trap based on imbalanced scattering rates consider an atom displaced from the center of the trap to a position $z=z^{\prime}$. At this location the magnetic field causes the Zeeman sublevel $m_{J}=-1$ to be brought closer into resonance with the atomic transition while bringing the $m_{J}=1$ sublevel further from resonance. The detuning of each of these states from resonance is indicated by $\Delta_{-}$and $\Delta_{+}$respectively, with $\Delta_{+}>\Delta_{-}$. Transition selection rules then indicate that the atom will scatter at a greater rate from the $\sigma^{-}$beam, pushing it back towards the center. If the atom is displaced in the opposite direction then the Zeeman shift in the sublevels will cause it to scatter preferentially out of the $\sigma^{+}$beam, again pushing back towards the center. Of course with the 3-D geometry shown in Fig. 2.10 any displacement from the trap center will lead to a restoring force. Additionally, the red detuning of the beams causes the atoms to cool according to the discussion of optical molasses, therefore MOTs both confine and cool atomic ensembles.

We can describe the simple 1-D situation more quantitatively by including the Zeeman shift into Eq. 2.78, which was used to describe optical molasses

$$
F_{M O T}=F_{\text {scatt }}^{\sigma^{+}}\left[\omega-k v-\left(\omega_{0}+\beta z\right)\right]+F_{\text {scatt }}^{\sigma^{-}}\left[\omega+k v-\left(\omega_{0}+\beta z\right)\right],
$$

where $\beta$ is the magnetic field gradient. This can be approximated near the 
trap center through a Taylor expansion yielding

$$
F_{M O T} \approx-2 \frac{\partial F}{\partial \omega} k v+2 \frac{\partial F}{\partial \omega_{0}} \beta z
$$

where the Zeeman shift at a displacement $z$ is

$$
\beta z=\frac{g \mu_{B}}{\hbar} \frac{d B}{d z} z
$$

As before, the scattering force (Eq. 2.77) depends on the frequency detuning $\Delta=\omega-\omega_{0}$, so $\partial F / \partial \omega_{0}=-\partial F / \partial \omega$ so that these two terms can be combined and written as

$$
F_{M O T}=-2 \frac{\partial F}{\partial \omega}(k v+\beta z)
$$

This can be brought into a particularly simple form by introducing the variable $\alpha$ originally defined in the discussion of optical molasses

$$
F_{M O T}=-\alpha v-\frac{\alpha \beta}{k} z
$$

This form emphasizes that the imbalance in the scattering rates caused by the Zeeman shift in energy levels leads to a restoring force with a spring constant $\alpha \beta / k$. In typical experimental situations the atom undergoes strongly over-damped motion.

\subsection{Branching Ratios}

During the course of the single-photon cooling process ${ }^{87} \mathrm{Rb}$ atoms are excited from the $\left|F=2, m_{F}=2\right\rangle$ state into the $\left|F^{\prime}=1, m_{F}=1\right\rangle$ state from where they spontaneously decay. Electric dipole selection rules, $\Delta F=$ 
$0, \pm 1$ and $\Delta m_{F}=0, \pm 1$, allow excited atoms to decay into any of the states $\left|F=2, m_{F}=0,1,2\right\rangle$ and $\left|F=1, m_{F}=0,1\right\rangle$. Not all of these final state are trappable so knowledge of the spontaneous decay branching ratios is important to aid in understanding the efficiency of this cooling technique. The relative decay rates can be calculated with the help of the Wigner-Eckart theorem [51, 72-74] which states that the matrix element of an irreducible tensor operator $T_{q}^{\kappa}$ between states of a general angular momentum basis is given by the product of a constant independent of the magnetic quantum numbers $\left(m, m^{\prime}, q\right)$ and an appropriate Clebsch-Gordan coefficient [58]:

$$
\left\langle\xi^{\prime}, j^{\prime}, m^{\prime}\left|T_{q}^{\kappa}\right| \xi, j, m\right\rangle=\frac{\left\langle\xi^{\prime}, j^{\prime}|| T^{\kappa}|| \xi, j\right\rangle}{\sqrt{2 j^{\prime}+1}}\left\langle j, m, \kappa, q \mid j^{\prime}, m^{\prime}\right\rangle
$$

where $\kappa$ is the rank of the tensor operator and $q$ labels its component in the spherical basis. The quantity $j$ represents a general angular momentum and $m$ is its projection along the quantization axis. The quantity indicated by the double bars

$$
\left\langle\xi^{\prime}, j^{\prime}\left\|T^{\kappa}\right\| \xi, j\right\rangle
$$

is known as a reduced matrix element.

The value of the Wigner-Eckhart theorem is that it allows the factorization of matrix elements into two terms, one of which, the reduced matrix element, depends only on the physical observable of interest and the other, the Clebsch-Gordan coefficient, depends only on the orientation of the physical observables with respect to the quantization axis. This is extremely useful because if one is able to find the value of one matrix element, then this the- 
orem can be used to find all of the others which differ only in the values of $m, m^{\prime}$ and $q$.

We can use the Wigner-Eckhart theorem to factor the electric dipole transition matrix element between two hyperfine states into two terms.

$$
\left\langle F, m_{F}\left|\mu_{q}\right| F^{\prime}, m_{F}^{\prime}\right\rangle=\left\langle F|| \mu|| F^{\prime}\right\rangle\left\langle F, m_{F} \mid F^{\prime}, 1, m_{F}^{\prime}, q\right\rangle
$$

This can be rewritten using the more symmetrical Wigner $3-j$ symbols which are related to the Clebsch-Gordan coefficients in the following way [75-77]

$$
\left\langle F, m_{F} \mid F^{\prime}, 1, m_{F}^{\prime}, q\right\rangle=(-1)^{F^{\prime}-1+m_{F}} \sqrt{2 F+1}\left(\begin{array}{ccc}
F^{\prime} & 1 & F \\
m_{F}^{\prime} & q & -m_{F}
\end{array}\right)
$$

as

$$
\left\langle F, m_{F}\left|\mu_{q}\right| F^{\prime}, m_{F}^{\prime}\right\rangle=\left\langle F|| \mu|| F^{\prime}\right\rangle(-1)^{F^{\prime}-1+m_{F}} \sqrt{2 F+1}\left(\begin{array}{ccc}
F^{\prime} & 1 & F \\
m_{F}^{\prime} & q & -m_{F}
\end{array}\right) .
$$

This reduced matrix element can be further reduced by using the WignerEckart theorem once more to factor out the $F$ and $F^{\prime}$ dependence into a Wigner $6-j$ symbol.

$$
\left\langle F\|\mu\| F^{\prime}\right\rangle=\left\langle J\|\mu\| J^{\prime}\right\rangle(-1)^{F^{\prime}+J+1+I} \sqrt{\left(2 F^{\prime}+1\right)(2 J+1)}\left\{\begin{array}{ccc}
J & J^{\prime} & 1 \\
F^{\prime} & F & I
\end{array}\right\} .
$$

The values of the $3-j$ and $6-j$ symbols are tabulated in [78] and can also be found from the built in functions ThreeJSymbol[] and SixJSymbol[] in the program Mathematica.

These formulas can be used to find the spontaneous decay branching ratios from the excited state $\left|F^{\prime}=1, m_{F}=1\right\rangle$ which are shown in Fig. 2.12. In 
this figure only the relevant hyperfine manifolds are shown. The decay modes

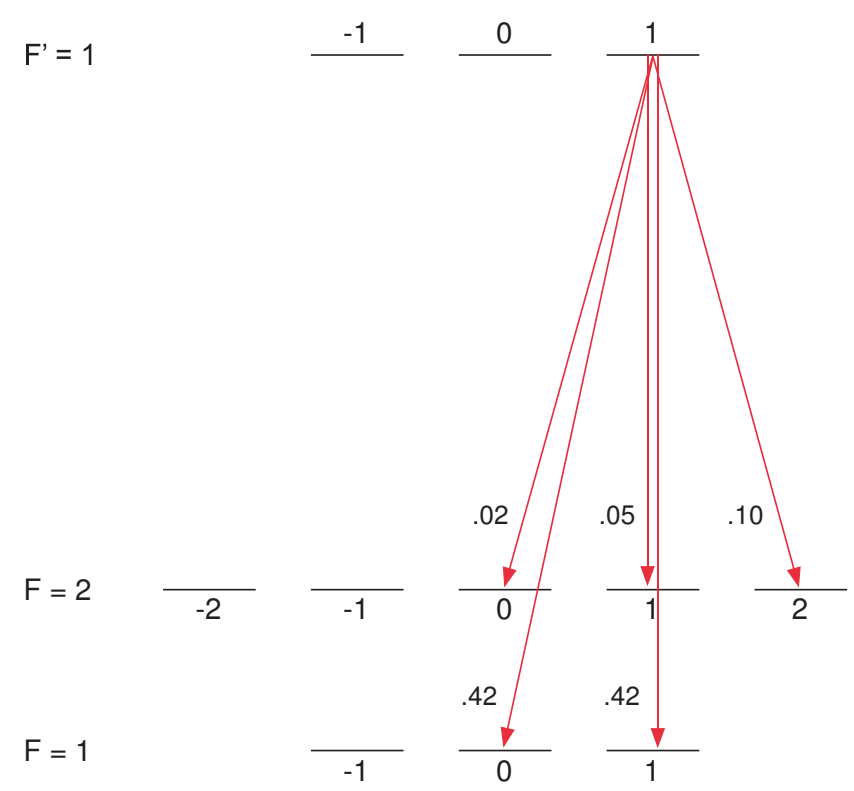

Figure 2.12: Decay mode branching ratios for an atom decaying from the $\left|F^{\prime}=1, m_{F}=1\right\rangle$ state. The allowed spontaneous electric dipole transitions are indicated by red arrows. The value next to each arrow indicates the relative strength of each transition.

allowed by a spontaneous electric dipole transitions from the $\left|F^{\prime}=1, m_{F}=1\right\rangle$ state are indicated by red arrows. The value next to each arrow indicates the relative strength of the transition. When single-photon cooling is applied to ${ }^{87} \mathrm{Rb}$ only those atoms which decay into the $\left|F=1, m_{F}=0\right\rangle$ state are considered when calculating quantities related to the cooling process, such as the final phase space density.

The absolute value of any $D_{2}$ transition can be found as a multiple of $\left\langle J=1 / 2\|\mu\| J^{\prime}=3 / 2\right\rangle$ which is determined from the natural lifetime of the 
excited state through the relation

$$
\frac{1}{\tau}=\frac{\omega_{0}^{3}}{3 \pi \epsilon_{0} \hbar c^{3}} \frac{2 J+1}{2 J^{\prime}+1}\left|\left\langle J|| \mu|| J^{\prime}\right\rangle\right|^{2}
$$

to have the value $3.58424(74) \times 10^{-29} \mathrm{C} \cdot \mathrm{m}$.

\subsection{Laser Frequency Control}

The laser beams used during the experiment can be roughly divided into two categories: those far from resonance with any transition in ${ }^{87} \mathrm{Rb}$ and those close to an atomic transition, specifically the $D_{2}$ transition. The absolute frequency of the laser beams which are far from resonance is not crucial (their role in optical dipole trapping is discussed elsewhere). There is a need however, to control the frequency of the near resonance laser beams. These beams are used for laser cooling and to induce a state change in ${ }^{87} \mathrm{Rb}$ atoms during the single-photon cooling process. This works only if the frequency of these beams are tuned correctly. Typically the frequency of these beams must be brought to within a few natural linewidths of the $D_{2}$ transition frequency. The frequency of this transition is $\approx 384 \mathrm{THz}$ while its natural linewidth is only $\approx 6 \mathrm{MHz}$. Therefore the frequency of these laser beams must be controlled to the $\sim 1$ $\mathrm{MHz}$ level, or to 1 part in $10^{8}$. A laser frequency locking scheme based around saturation absorption spectroscopy is use for this purpose.

\subsubsection{Doppler Broadening}

At room temperature, Doppler broadening is usually the dominant contribution to the observed width of lines in atomic spectra. For example, the 
$D_{2}$ transition in ${ }^{87} \mathrm{Rb}$ has a natural linewidth of $6 \mathrm{MHz}$ due to the process of radiative damping [70]. Doppler broadening smears this line out increasing its observed width to $\sim 0.5 \mathrm{GHz}$ at room temperature. The source of this broadening can be understood by considering the relationship between the angular frequency $\omega$ of radiation in the laboratory frame of reference and the angular frequency $\omega^{\prime}$ seen in a frame of reference moving at velocity $\vec{v}$

$$
\omega^{\prime}=\omega-\vec{k} \cdot \vec{v}
$$

where $\vec{k}$ is the wavevector of the radiation and has a magnitude $k=\omega / c$. This equation is correct to first order in $v / c$ and suffices in most situations, however higher order Doppler effects must be considered in extremely precise spectroscopic measurements. As indicated by the dot product, it is the component of velocity along the direction of $\hat{k}$ which is responsible for this shift. Therefore to simplify this notation we assume a $1-D$ geometry so that $\vec{k} \cdot \vec{v}=k v$. Figure 2.13 illustrates these ideas for a single atom moving with velocity $v$ to the right. This atom sees radiation traveling to the right at a decreased frequency and radiation traveling to the left at an increased frequency.

Consider now an ensemble which consists of atoms which absorb radiation at frequency $\omega_{0}$ in their rest frame, i.e. when $\omega^{\prime}=\omega_{0}$. Atoms with velocity $v$ will absorb radiation when the Doppler effect shifts the frequency into resonance

$$
\omega-\omega_{0}=k v .
$$

If we assume that the ensemble is in thermal equilibrium then the 


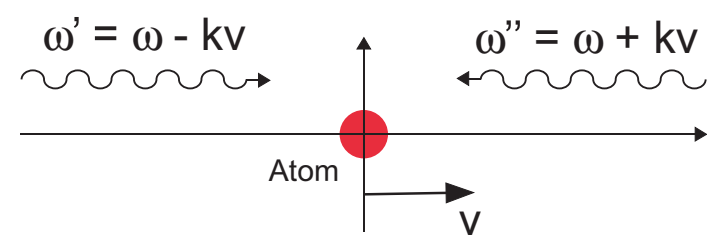

Figure 2.13: The Doppler effect shifts the observed frequency of radiation due to the atoms velocity. The atom travels to the right and sees radiation traveling to the right at a decreased frequency and radiation traveling to the left at an increased frequency.

velocity distribution is given by the $1-D$ Maxwell-Boltzmann distribution

$$
f(v) d v=\frac{1}{u \sqrt{\pi}} e^{-\frac{v^{2}}{u^{2}}}
$$

where $u=\sqrt{2 k_{B} T / m}$ is the most probable speed for atoms. The absorption profile $g(\omega)$ of this ensemble can be found by relating the velocity of the atoms with their corresponding absorption frequency using Eq. 2.97, the result being

$$
g(\omega)=\frac{c}{u \omega_{0} \sqrt{\pi}} e^{-\frac{c^{2}}{u^{2}}\left(\frac{\omega-\omega_{0}}{\omega_{0}}\right)^{2}} .
$$

This Doppler broadened profile has a Gaussian shape and a full width at half maximum (FWHM) $\Delta \omega_{D}$ of

$$
\Delta \omega_{D}=2 \omega_{0} \sqrt{\ln 2} \frac{u}{c}
$$

which for ${ }^{87} \mathrm{Rb}$ is $1.4 \times 10^{-6}$, limiting the resolution of spectroscopic measurements to approximately 1 part in $10^{6}$. Because we use a laser frequency locking scheme which is referenced to ${ }^{87} \mathrm{Rb}$ transition lines, the resolution with which we resolve these transitions places a limit on our ability to control the frequency of the lasers. The needed resolution is 1 part in $10^{8}$ so we must use a spectroscopic technique which suppresses the effect of Doppler broadening. 


\subsubsection{Saturation Absorption Spectroscopy}

Saturation absorption spectroscopy is a nonlinear Doppler-free spectroscopic technique $[18,79]$. The standard setup for this technique is shown in Fig. 2.14. A beam splitter separates a beam into a weak probe beam and a

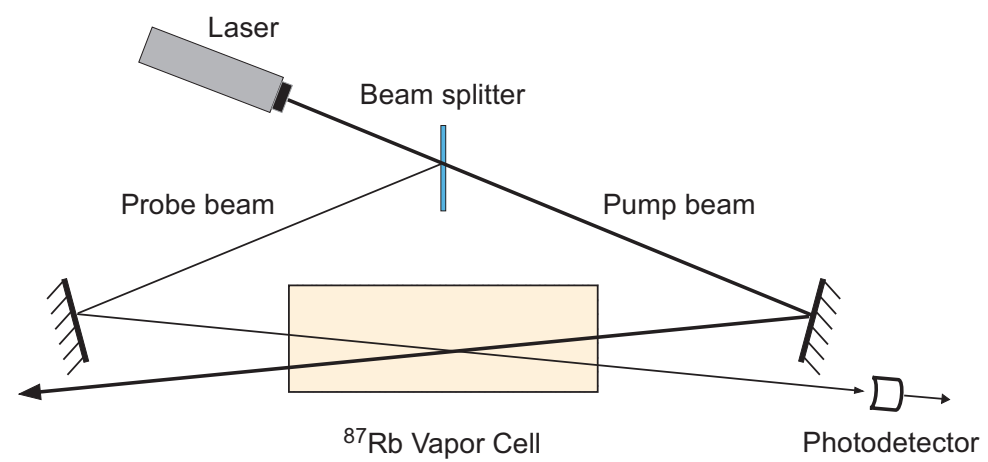

Figure 2.14: A typical saturation absorption spectroscopy setup applied to ${ }^{87} \mathrm{Rb}$. A laser is divided unequally into two beam paths. An intense pump beam overlaps a weaker counter-propagating probe beam in an atomic vapor cell. The transmission intensity of the probe beam is monitored with a photodiode as a function of laser frequency.

strong pump beam which travel in opposite directions and overlap in a vapor cell. The intensity of the pump beam is typically larger than the saturation intensity of the transition $\left(I_{\text {pump }} \gtrsim I_{\text {sat }}\right)$, while the probe beam is much less intense $\left(I_{\text {probe }} \ll I_{\text {sat }}\right)$. The intensity of the transmission of the probe beam is monitored as a function of laser frequency. Figure 2.15(a) shows a typical result of such a measurement in the absence of a pump beam when probing a single transition. The result is a Doppler broadened absorption profile of width $\Delta \omega_{D}$. 

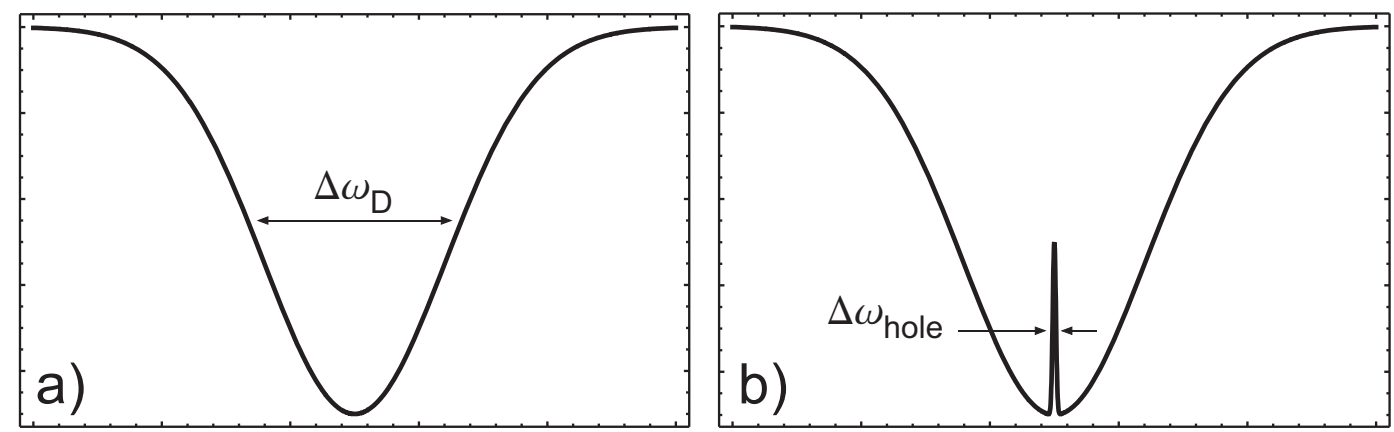

Figure 2.15: Spectrum obtained (a) without and with (b) the pump beam. (a) With no pump beam present the resulting spectrum is dominated by Doppler broadening and has a FWHM of $\Delta \omega_{D}$. (b) When the pump beam is present a narrow peak of FWHM $\Delta \omega_{\text {hole }}$ is seen at the center of the Doppler broadened spectrum.

If the pump beam is present the result of the same scan shows a narrow peak in the center of the wide Doppler profile, Fig. 2.15(b). This can be understood in terms of the action of the pump beam. It interacts with atoms that have a velocity $v=\left(\omega-\omega_{0}\right) / k$ and excites many of them into the upper level, removing them from the lower level, in a process known as hole burning [79]. The width of the hole burnt into the lower level is equal to the powerbroadened homogeneous linewidth

$$
\Delta \omega_{\text {hole }}=\Gamma\left(1+\frac{I_{\text {pump }}}{I_{\text {sat }}}\right)^{1 / 2}
$$

If the frequency of the laser beam is off resonance then this has no effect on the transmission of the probe beam because it interacts with atoms with a velocity $v=-\left(\omega-\omega_{0}\right) / k$. However, if the laser beam is resonant with the transition then both beams interact with atoms with velocity $v=0$ and the 
ground state depletion due to the pump beam reduces the absorption of the probe beam producing the sharp peak in Fig. 2.15(b). The width of this peak is given by Eq. 2.101 and is much smaller than the Doppler width.

If there are several excited states separated by less than the Doppler width which share a common ground state then the spectrum obtained displays cross-over resonances. This situation is depicted in Fig. 2.16. Cross-over resonances appear midway between true transitions and result from atoms moving with velocity such that one transition is resonant with the pump beam while the other transition is resonant with the probe beam.
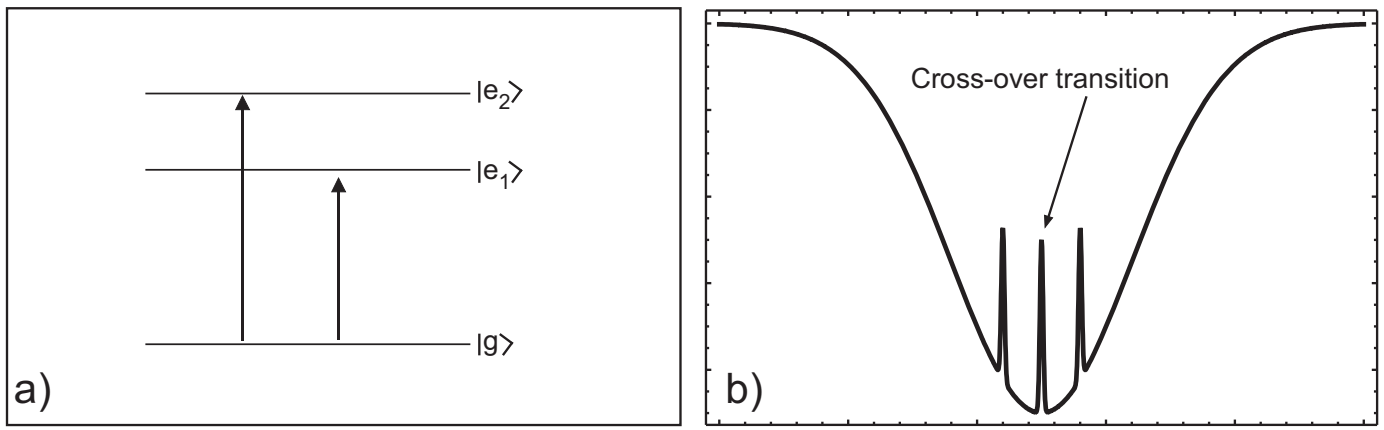

Figure 2.16: (a) An atom has two excited states within the Doppler width which share a common ground state. (b) The resulting spectrum obtained with the saturated absorption technique has in addition to two peaks corresponding to the center of each transition a third peak known as a "cross-over resonance." This peak comes from atoms with velocity such that the pump beam is resonant with one transition, while at the same time the probe beam is resonant with the other transition. This always occurs exactly between the two real transition frequencies and due to this symmetry the appearance of cross-over resonances usually does not present added ambiguity in interpreting a spectrum. 


\subsubsection{Laser Frequency Lock}

The spectrum obtained from saturation absorption spectroscopy can be used to lock the frequency of a laser using a conventional PID feedback loop. To keep the discussion simple, consider the signal obtained from a single transition such as that shown in Fig. 2.17. This signal can be obtained by

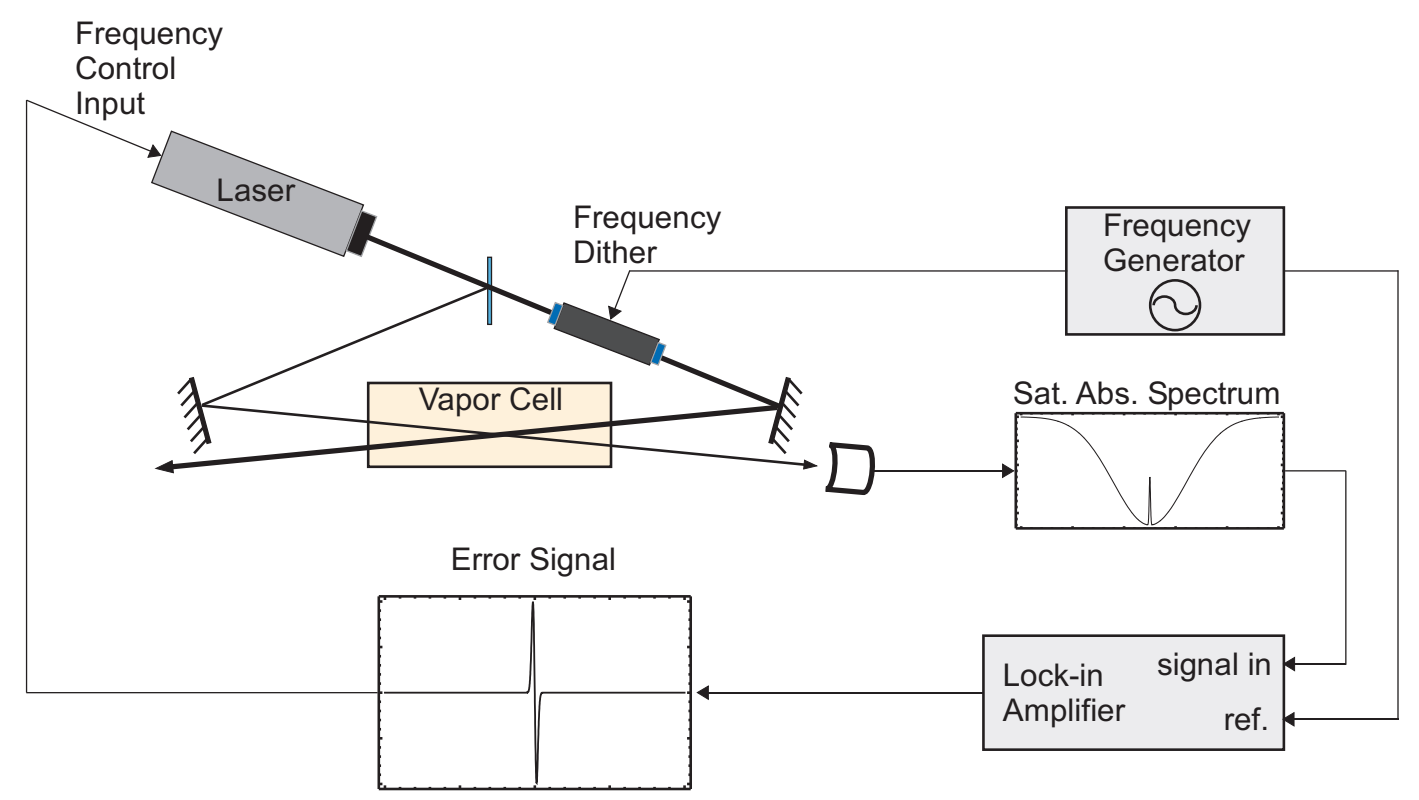

Figure 2.17: A typical layout of a saturation absorption spectroscopy setup used to lock the frequency of a laser. The frequency of a laser beam is slowly swept over an atomic transition and the spectrum is observed using a saturation absorption spectroscopic technique. If a frequency dither is put on the pump beam and the resulting signal is mixed with the dithering frequency in a lock-in amplifier, the resulting signal is a Doppler background free error signal, which is the derivative of the narrow central peak of the original signal. This signal can be used an an "error signal" to lock the frequency of a laser.

slowly sweeping the frequency of the laser up and down. The wide Doppler 
broadened background can be removed from this signal by adding a fast frequency dither to the pump beam and then mixing the saturation absorption spectrum with the dither frequency $\omega_{\text {dither }}$ in a lock-in amplifier (see Fig. 2.17). The removal of the Doppler broadened background occurs because if the laser beam is off resonance then the pump and probe beam do not interact and a frequency dither of the pump beam does not induce an intensity modulation in the probe beam at $\omega_{\text {dither }}$. The lock-in amplifier output is therefore zero for all such laser frequencies. If however the laser frequency is near resonance then a frequency dither of the pump beam does induce a modulation in the intensity of the probe beam at $\omega_{\text {dither }}$. Furthermore, the amplitude of the modulation is proportional to the slope of the saturation absorption spectrum so the output of the lock in amplifier is the Doppler background free derivative of the spectrum. This curve is perfect for use as an "error signal" in a laser frequency feedback loop because it is zero on resonance and has an opposing sign on either side of resonance.

This scheme is used to lock our near resonance laser during the experiment. The detailed layout of our locking scheme can be found in Ch. 3 .

\subsection{Imaging}

During the course of the experiment the atomic cloud is imaged so that we can extract information about the ensemble, such as its density distribution, total atom number and temperature. We use two atomic imaging techniques to accomplish this. The first, absorption imaging, relies on the atomic sample 
scattering photons out of a resonant beam to form a "shadow" on a CCD camera. The second technique, fluorescent imaging, places the atomic sample in optical molasses and a portion of the resulting fluorescence is captured and imaged. These two imaging techniques are somewhat complementary because absorption imaging yields spatial information while fluorescence imaging is more sensitive to atom number.

\subsubsection{Absorption Imaging}

Absorption imaging can be understood by imagining a near- or onresonance laser beam passing through an atomic sample. As discussed in Sec. 2.5.2.2, atoms in the sample will each scatter photons out of the laser beam at a rate

$$
R_{\text {scatt }}=\frac{\Gamma}{2} \frac{\left(I / I_{\text {sat }}\right)}{1+4(\Delta / \Gamma)^{2}+\left(I / I_{\text {sat }}\right)} .
$$

We can use this equation to define the atomic scattering cross section $\sigma$ as the power scattered by each atom per incoming light intensity $\left(\sigma=R_{\text {scatt }} \hbar \omega / I\right)$

$$
\sigma=\frac{\sigma_{0}}{1+4(\Delta / \Gamma)^{2}+\left(I / I_{s a t}\right)}
$$

where

$$
\sigma_{0}=\frac{\hbar \omega \Gamma}{2 I_{s a t}}
$$

is the on-resonance, low saturation cross section. As the laser beam passes through the atomic cloud scattering will reduce its intensity according to Beer's law

$$
\frac{d I}{d z}=-\sigma n(x, y, z) I
$$


where $n$ is the number density of atoms. Direct integration of this equation yields the transverse intensity profile of the beam at a position $z$.

$$
I(x, y)=I_{0}(x, y) e^{-\sigma \int n(x, y, z) d z}
$$

This equation shows us that the intensity of the beam after passing through the atomic sample is proportional to the negative exponential of the scattering cross section multiplied by the integrated atomic column density. Therefore by measuring $I(x, y)$ and $I_{0}(x, y)$ it is possible to extract the atomic column density. $I(x, y)$ and $I_{0}(x, y)$ are measured by a CCD camera, the former is taken with the atoms of interest present in the beam path and the latter is taken with no atoms present. A computer then calculates the optical density of the sample, defined as

$$
D_{\text {opt }}(x, y)=-\ln \left(\frac{I(x, y)}{I_{0}(x, y)}\right)=\sigma \int n(x, y, z) d z,
$$

to yield a quantity directly proportional to the integrated column density. This quantity is easily manipulated numerically to give the total number of atoms $N$ present in the sample.

$$
N=\iint D_{o p t}(x, y) d x d y
$$

This imaging technique is also used to probe the temperature of atomic samples through the Time-of-Flight (TOF) method [80,81]. The idea is to release an atomic sample from all trapping potentials and then measure its subsequent expansion. In the absence of inter-atomic interactions, the released atoms follow ballistic trajectories with velocities proportional to their 
momentum at the time of release. The final atomic distribution is therefore a convolution of the initial atomic distribution with the initial momentum distribution.

This can be made more concrete by considering atoms originating from $\vec{r}_{0}$ and arriving at $\vec{r}$ after a time-of-flight $t$. If we assume that the velocity distribution for these particles is $\zeta(\vec{v})$ then we expect to find the number of atoms in a volume element $d^{3} r$ located at position $\vec{r}$ at time $t$ to be [80]

$$
d n=G\left(\vec{r}, t ; \vec{r}_{0}\right) d^{3} r=\zeta\left(\vec{v}=\left[\vec{r}-\vec{r}_{0} / t\right]\right) d^{3} v,
$$

where $G\left(\vec{r}, t ; \vec{r}_{0}\right)$ is the boundary Green's function describing the evolution of the spatial distribution of the atoms. If the atoms have a 3-D MaxwellBoltzmann velocity distribution initially, then the Green's function is given by $[80]$

$$
G\left(\vec{r}, t ; \vec{r}_{0}\right)=\left(\frac{m}{2 \pi k_{B} T t^{2}}\right)^{3 / 2} e^{-m\left|\vec{r}-\vec{r}_{0}\right|^{2} / 2 k_{B} T t^{2}} .
$$

If we assume that the atomic sample has a Gaussian spatial distribution initially, given by

$$
n_{0}(\vec{r})=\frac{1}{\left(2 \pi \sigma_{n}^{2}\right)^{3 / 2}} e^{-r^{2} / 2 \sigma_{n}^{2}},
$$

then we can solve for the spatial distribution after an expansion time $t$ by evaluating

$$
n(\vec{r}, t)=\int G\left(\vec{r}, t ; \vec{r}_{0}\right) n_{0}\left(\vec{r}_{0}\right)_{0} d^{3} r_{0}
$$

The result is

$$
n(\vec{r}, t)=\frac{1}{\left(2 \pi \sigma_{c}^{2}\right)^{3 / 2}} e^{-r^{2} / 2 \sigma_{c}^{2}},
$$


where

$$
\sigma_{c}^{2}=\sigma_{n}^{2}+\frac{k_{B} T t^{2}}{m}
$$

By taking multiple images of the atomic cloud as it expands and fitting the measured widths to this expression, the temperature can be extracted. Examples of this technique applied to our atomic samples of ${ }^{87} \mathrm{Rb}$ are given in Ch. 4.

\subsubsection{Fluorescence Imaging}

In addition to using absorption imaging, we also use the technique of fluorescence imaging to determine the total number of atoms present in our sample. The idea is quite straightforward: atoms are placed into optical molasses which slows their motion via photon scattering as discussed in Sec. 2.5.2.2. The scattered photons are partially collected on a CCD and used to estimate the number of atoms taking part in the scattering process. The atom number can be estimated with help from Eq. 2.76 to be [43]

$$
N_{\text {atom }}=\frac{8 \pi\left[1+4(\Delta / \Gamma)^{2}+\left(6 I_{0} / I_{\text {sat }}\right)\right]}{\Gamma\left(6 I_{0} / I_{\text {sat }}\right) t_{\text {exp }} \eta_{\text {count }} d \Omega} N_{\text {counts }}
$$

where $I_{0}$ is the intensity in each of the six molasses beams, $t_{\text {exp }}$ is the CCD exposure time, $\eta_{\text {counts }}$ is the CCD efficiency in (counts/photon) and $d \Omega$ is the solid angle of photon collection.

In the experiment this technique is used when the atom number is too low to yield an absorption signal or when we are interested solely in atom 
number and not the spatial profile of the atomic cloud. This technique is so sensitive it can be used to detect a single atom [82]. 


\section{Chapter 3}

\section{Experimental Apparatus}

This chapter discusses the apparatus used to perform the experiments discussed in this dissertation. It begins with a description of the vacuum chamber used to isolate our atoms from the environment. Next, a discussion of the laser systems used to control and confine the atoms is presented. This portion starts with a description of the near-resonance lasers which are used to cool, confine and control the internal state of the atoms. Then, a discussion of the far-detuned laser used to form a conservative optical dipole trap for the atoms is given. Next the coils used to create the magnetic field for both the MOT and magnetic trap are discussed. Finally, the geometries of the vertical and horizontal imaging systems are outlined. Because the apparatus has been explained in detail elsewhere [83-85], I will only discuss the most relevant aspects, giving references to sources of more detail for the interested reader.

\subsection{Vacuum Chamber}

The vacuum chamber is designed to operated in a double MOT configuration after the design given in reference [86]. The idea behind this configuration is that there are two desirable but competing features one needs in a 
vacuum chamber used for atomic experiments. The two features, rapid MOT loading and a long magnetic trap confinement time, are typically mutually exclusive because the former is favored by a high background vapor pressure while for the latter very low background pressures are required. The double MOT configuration solves this problem by separating the chamber into two regions, one of high pressure and one of low, connected by a differential pumping tube. Atoms are rapidly loaded in the high pressure region and then "pushed" with a resonant beam (the so-called push beam) into the low pressure region. To this end, our chamber consists of an upper region maintained at the rubidium vapor pressure, a lower chamber more than three orders of magnitude lower in pressure, and a middle chamber which houses the pumps required to maintain this pressure differential. The entire chamber is shown in Fig. 3.1. This photograph was taken during a vacuum bakeout so the chamber is away from the experiment, allowing a clear view of its elements. The three vacuum chamber sections are indicated in the photograph.

\subsubsection{Upper Chamber}

The upper chamber serves as a high pressure region for quickly accumulating ${ }^{87} \mathrm{Rb}$ atoms in a MOT which are then pushed into the lower chamber. This function is served with a simple design consisting of three main elements: a glass cell, a source of rubidium and a differential pumping tube connecting the upper and lower chamber, as shown in Fig. 3.2.

The glass cell is constructed from a rectangular section of Pyrex glass 


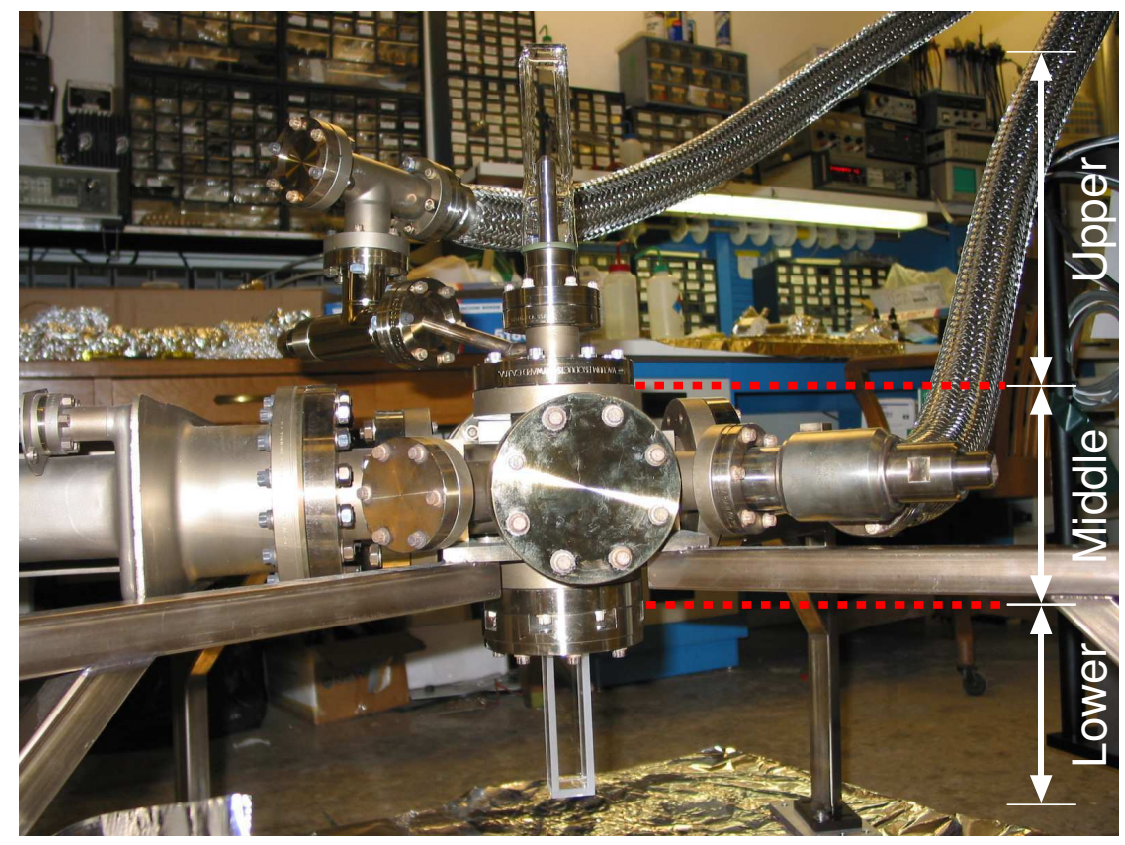

Figure 3.1: The vacuum chamber during a bakeout, away from the rest of the experimental apparatus. The upper, middle and lower portions of the chamber are marked.

and has the outer dimensions of $4^{\prime \prime} \times 1-1 / 4^{\prime \prime} \times 1-1 / 4^{\prime \prime}$. The chamber walls are $1 / 4^{\prime \prime}$ thick. The cell is connected to a $2-3 / 4^{\prime \prime} \mathrm{CF}$ steel flange through a graded glass-to-metal seal (Larson Electronics Glass Inc. SQ-150-F2). These two pieces were assembled by Technical Glass Inc. allowing the glass cell to be connected to standard vacuum components.

The 2-3/4" flange at the base of the glass cell is attached to a composite piece of vacuum hardware. This composite piece consists of a $2-3 / 4^{\prime \prime}$ rotatable half-nipple welded to a rotatable $4-1 / 2^{\prime \prime}$ blank flange. This piece serves three main purposes. First, it acts as a size adapter allowing the glass cell to be 

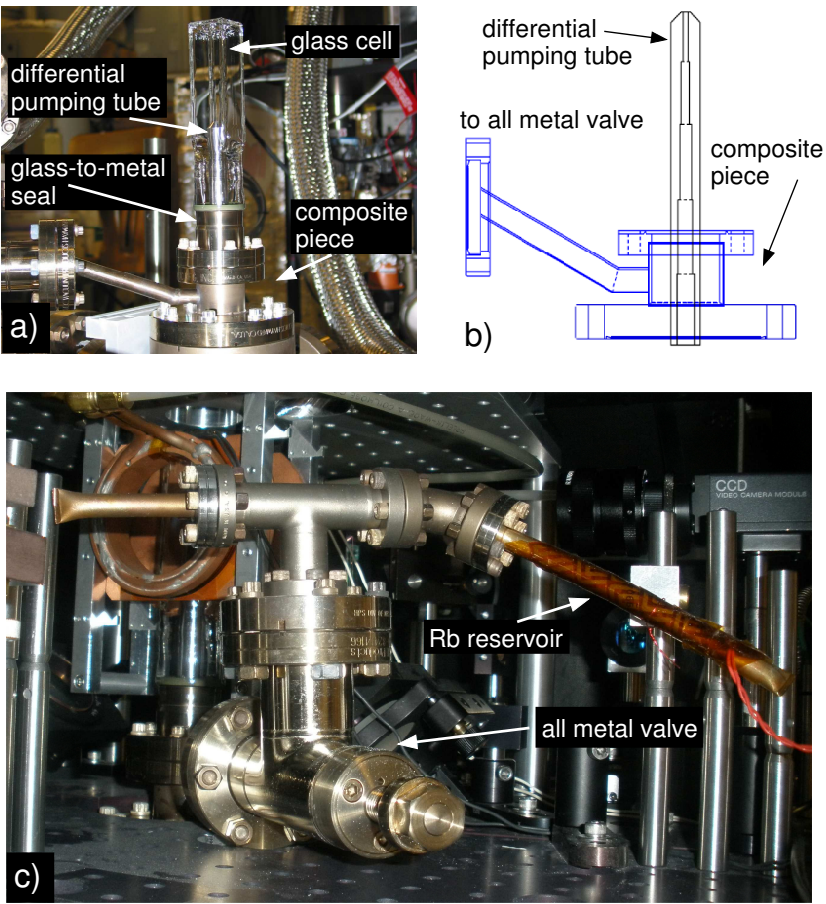

Figure 3.2: Views of the upper chamber. a) The glass cell connects to the composite piece through a glass-to-metal seal. The differential pumping tube is also attached to the composite piece. b) Schematic of the differential pumping tube and composite piece. The CF flange on the right connects to an all metal valve in the experiment. c) The all metal valve connects to the Rb reservoir.

attached to the middle chamber. Second, the differential pumping tube is welded to the composite piece such that the only connection between the upper and lower chamber is through this tube. Finally, a $1 / 2^{\prime \prime}$ tube is welded to the side of the nipple portion of the composite piece and serves as a path to introduce rubidium into our vacuum system.

A $2-3 / 4^{\prime \prime}$ flange is welded to the end of the $1 / 2^{\prime \prime}$ tube connected to the side of the nipple. The flange is attached to a $2-1 / 2^{\prime \prime}$ all metal valve from 
Nor-Cal Products Inc. (AMV-1502-CF). Attached to the metal valve via a zero length reducer is a $1-1 / 3^{\prime \prime}$ tee. Both sides of the tee have $1 / 2^{\prime \prime}$ copper pinch-off tubes (Huntington, CPT-133-050) attached. One of these was used as a connection port to a turbo-pump station during the vacuum bakeout. It was sealed when the bakeout was complete. The other pinch-off tube houses a glass ampule containing $\approx 200 \mathrm{mg}$ of Rb (ESPI, purity 3N5). The ampule was cracked from the outside by squeezing the copper tube with pliers when the pump down was complete but while the chamber was still attached to the turbo-pump station.

The $\approx 200 \mathrm{mg}$ piece of solid $\mathrm{Rb}$ contains the isotope of interest, ${ }^{87} \mathrm{Rb}$, at the natural abundance level of $\approx 28 \%$. One may wonder what role, if any, the more abundant isotope ${ }^{85} \mathrm{Rb}$ played in our experiment. The isotopic shift is sufficiently large so that ${ }^{85} \mathrm{Rb}$ is not resonant with the laser beams tuned near the $D_{2}$ transition in ${ }^{87} \mathrm{Rb}$. This means that ${ }^{85} \mathrm{Rb}$ is not captured in our MOTs. However, the two isotopes can collide resulting in magnetic trap loss. The combined vapor pressure of both isotopes determines the collision rate, and hence the lifetime of atoms in the magnetic trap. In this sense the presence of ${ }^{85} \mathrm{Rb}$ has a somewhat detrimental effect.

The pressure in the upper chamber is determined by the vapor pressure of solid rubidium at room temperature which can be approximated by Eq. 2.1

$$
\log _{10} P_{v}=2.881+4.857-\frac{4215}{T} .
$$

The experiment resides in a room servo-looped to maintain a temperature 
of $22^{\circ} \mathrm{C}$. The expected vapor pressure is therefore $\approx 3 \times 10^{-7}$ torr. This pressure is sufficiently high to rapidly load a MOT in the upper chamber. This MOT serves as a reservoir of atoms which are pushed through the differential pumping tube and recaptured in the lower MOT.

The differential pumping tube maintains a pressure differential between the upper and lower chamber allowing long magnetic trap lifetimes in the lower chamber. The differential pumping tube is formed from a 6-3/4" long 304 stainless steal tube with an outer diameter of approximately $1 / 2^{\prime \prime}$. The inner diameter increases in five steps from $1 / 8^{\prime \prime}$ at the top to $3 / 8^{\prime \prime}$ at the bottom. This internal taper was made to allow room for the push beam to diverge while still maintaining a sufficiently low conductance, which is calculated to be $C \approx 52 \mathrm{~mL} / \mathrm{s}$ for this tube [87]. The pumping speed in the lower chamber is $75 \mathrm{~L} / \mathrm{s}$ resulting in a pressure differential of approximately 1500 between the upper and lower chambers [87]. The expected pressure in the lower chamber is therefore approximately $2 \times 10^{-10}$ torr.

The top of the differential pumping tube is cut at $20^{\circ}$ to allow diagonal MOT beams to pass unclipped. A MOT is formed in the upper chamber from three pairs of counter-propagating near-resonant beams and two current carrying wire coils, as discussed in Sec. 2.5.2.4.

\subsubsection{Middle Chamber}

The middle-chamber is located directly below the upper-chamber and serves as a hub to attach the vacuum equipment needed to pull, maintain, 
and monitor the necessary vacuum level in the lower-chamber. This section is necessary because both the upper and lower chambers primarily consist of glass cells, greatly restricting the number of possible connections to other vacuum components. This portion of the vacuum chamber dates back to prerubidium days when it was used in experiments performed with cesium. A detailed description of this vacuum chamber section can be found in several dissertations discussing those experiments [88-90] so only a brief overview will be given here.

The middle chamber is a modified six-way cross with $4-1 / 2^{\prime \prime}$ CF flanges. The upper and lower flanges are connected to the upper and lower chamber respectively. The modification of this chamber involved the addition of four half-nipples, each 1-1/2" long with 2-3/4" non-rotatable CF flanges attached to one end. These are positioned in between the original $4-1 / 2^{\prime \prime}$ ports in a plane horizontal to the ground. While this arrangement allows for eight connections to be made to this chamber (not including the connection to the upper- and lower- chambers), only four are currently in use.

Attached to one 4-1/2" flange is an ion pump (Varian 919-0103) with a pumping speed rated at $75 \mathrm{~L} / \mathrm{s}$. The pump is supplied with power by a VacIon pump control unit (model 921-0062) operating at $6 \mathrm{kV}$. A titanium sublimation pump is attached to a second $4-1 / 2^{\prime \prime}$ flange. This unit has a pumping speed of $300 \mathrm{~L} / \mathrm{s}$ under normal operating conditions. A hot-cathode, nude BayardAlpert style ion gauge is attached to one of the 2-3/4" flanges. This ion gauge operates in the $10^{-3}$ to mid- $10^{-10}$ torr range limiting its usefulness once 
operating pressures of high $10^{-11}$ torr have been reached. Finally, an all metal valve is attached to the $2-3 / 4^{\prime \prime}$ flange opposite to that used for the ion gauge. This valve is used during vacuum bakeouts to connect the middle-chamber to the turbo-pump station. The middle-chamber and the attached elements discussed here are shown in Fig. 3.3 in a top-down photograph of the vacuum chamber.

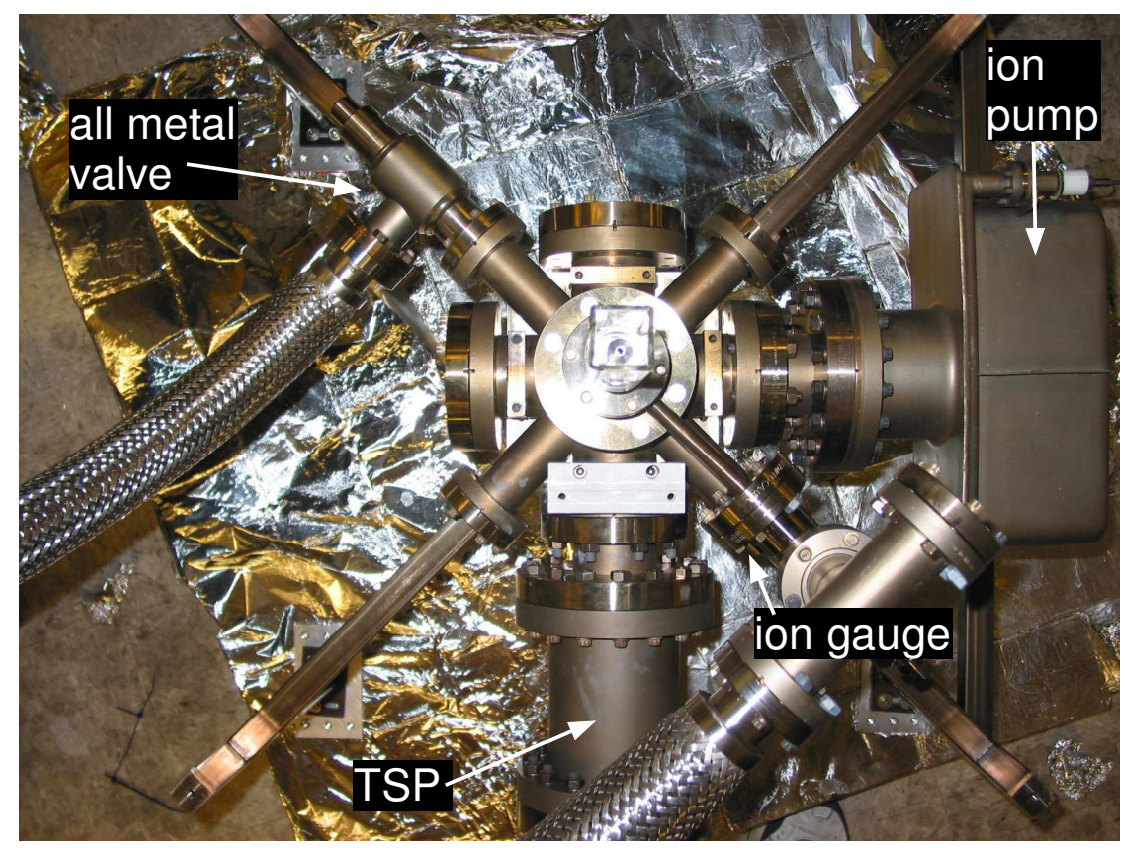

Figure 3.3: Top-down photograph of the vacuum chamber. Pictured attachments are the ion pump, titanium sublimation pump (TSP), ion gauge and an all metal valve used during the bakeout process. 


\subsubsection{Lower Chamber}

The lower chamber is where all of the experiments take place. It has been designed to provide an ultra-high vacuum (UHV) environment with excellent optical access. Like the upper chamber, the lower chamber consists primarily of a glass cell, but because the optical interaction and probe beams must pass through the walls of this cell it is of much higher optical quality.

The lower chamber is constructed from a rectangular glass cell attached to a glass disc, shown in Fig. 3.4. Both of these pieces are made from the

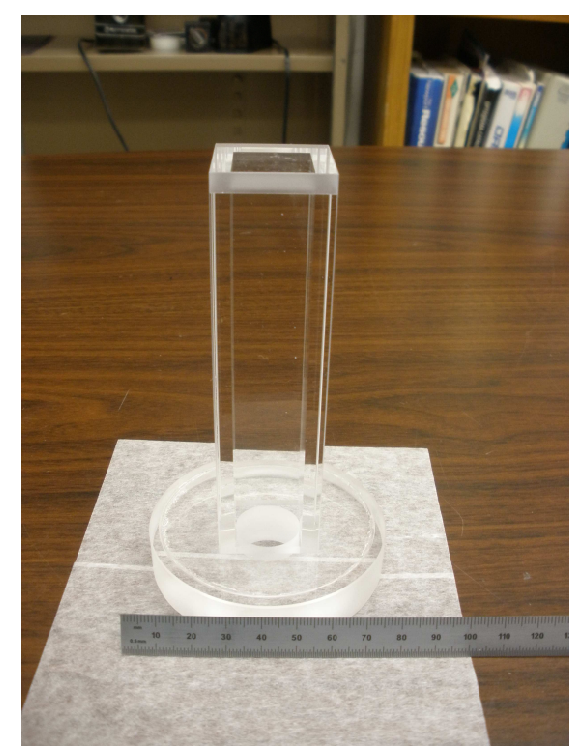

Figure 3.4: The lower glass cell is constructed from a rectangular and a disc shaped piece.

material Spectrosil, a "UV grade" synthetic fused silica with excellent optical properties. The rectangular cell has the outer dimensions $30 \times 30 \times 115 \mathrm{~mm}$ 
and has walls $5 \mathrm{~mm}$ thick. The glass disc has a diameter of $75 \mathrm{~mm}$, a thickness of $17 \mathrm{~mm}$ and a hole through its center with a diameter of $20 \mathrm{~mm}$ which allows passage between it and the middle chamber. The piece was prepared and assembled by Hellma Cells Inc. using a remarkable technique. Each of the glass plates used in constructing the cell was polished and optically contacted. The cell was then heated to just below the material melting point, fusing the pieces together without the use of any cement or adhesive. This technique produces very high optical quality cells which are free of any material (i.e. adhesive) which could potentially be UHV incompatible.

The all glass lower cell is connected to the middle chamber through the use of spring energized seals manufactured by Garlock Helicoflex (H-307330). These seals work via the elastic deformation of a metal jacket surrounding a close-wound helical spring. The metal jacket is chosen to have a greater ductility than both the glass cell and steel chamber it connects. The seals we used have two small ridges on the top and bottom which concentrate the compression load in small region (see Fig. 3.5).

The seals are available with jackets made from a variety of materials. The jacket used in the experimental apparatus was made from silver. While a good seal was formed, this product was found to damage the glass cell. The damage is visible in Fig. 3.4 as a white ring on the disc shaped section of the cell. After the damage was discovered we experimented with aluminum jacketed seals (H-307592) and found that they seal equally well and do not damage the glass substrate. 

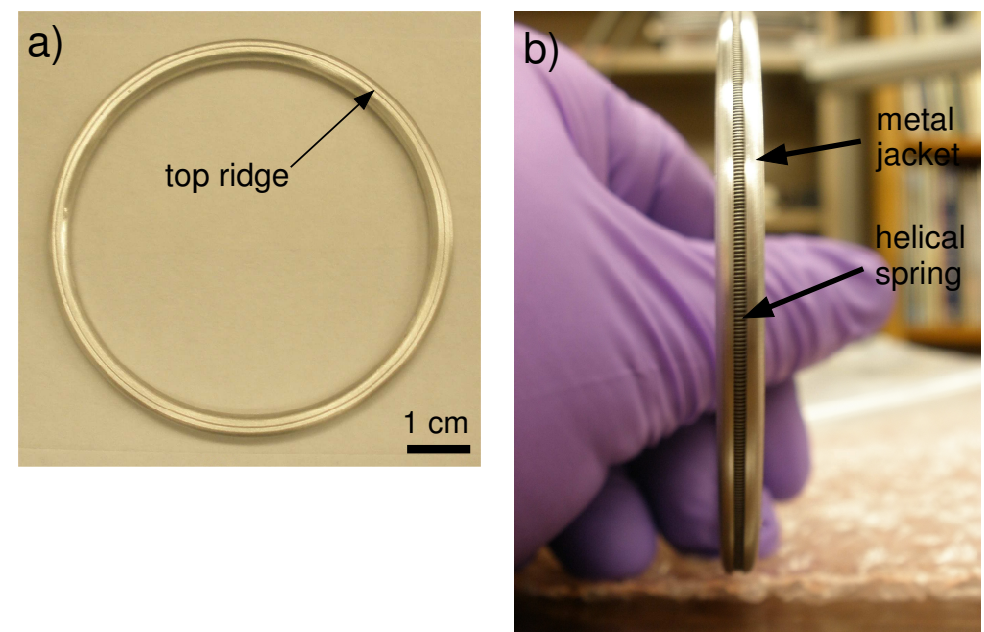

Figure 3.5: A Helicoflex seal. a) The narrow ridges along the top and bottom concentrate the compression load, facilitating the sealing process. b) A metal jacket surrounds a helical spring to form this type of sealing device.

\subsection{Lasers}

There were a total of seven lasers used during the course of the experiments described in this dissertation. Five of these lasers have frequencies near the ${ }^{87} \mathrm{Rb} D_{2}$ transition and were used to capture, cool, detect, and manipulate the internal state of the atoms. I refer to these as near-resonance lasers. The other two lasers have frequencies far detuned from any atomic resonance in $\mathrm{Rb}$ and were used to form optical dipole traps to contain the atoms. A description of each laser and its use in the experiment is given in the next several sections. 


\subsubsection{Near-Resonance Lasers}

Laser beams tuned near the resonance of the ${ }^{87} \mathrm{Rb} D_{2}$ transition are used for a variety of purposes in our experiment. This includes forming MOTs in conjunction with a magnetic field gradient. The frequency of these beams is typically about $15 \mathrm{MHz}$ to the red of the $|F=2\rangle \rightarrow\left|F^{\prime}=3\right\rangle$ transition. Because ${ }^{87} \mathrm{Rb}$ atoms occasionally decay into the $|F=1\rangle$ manifold, where they are no longer resonant with the MOT beams, a repump beam is introduced into the system to place them back in the cooling cycle. The repump beam is resonant with the $|F=1\rangle \rightarrow\left|F^{\prime}=2\right\rangle$ transition and serves to deplete atoms from the $|F=1\rangle$ ground state. To cool atoms via optical molasses (Sec. 2.5.2.2) we use beams tuned $50 \mathrm{MHz}$ to the red of the $|F=2\rangle \rightarrow\left|F^{\prime}=3\right\rangle$ transition. To image and push atoms from the upper MOT to the lower MOT a beam resonant with the $|F=2\rangle \rightarrow\left|F^{\prime}=3\right\rangle$ transition is used. To place atoms in a magnetically trappable state they are optically pumped by a laser resonant with the $|F=2\rangle \rightarrow\left|F^{\prime}=2\right\rangle$ transition. Finally, to change the internal state of the atoms from $\left|F=2, m_{F}=2\right\rangle$ to $\left|F=1, m_{F}=0\right\rangle$ during the single-photon cooling process a depopulation beam is used. This beam is tuned $35 \mathrm{MHz}$ to the red of the $|F=2\rangle \rightarrow\left|F^{\prime}=1\right\rangle$ transition. A diagram

of each of these beams in relation to the hyperfine structure of the ${ }^{87} \mathrm{Rb} D_{2}$ transition is given in Fig. 3.6.

To generate the required frequencies and optical powers needed to perform these tasks, a total of five diode lasers are used. Two of these diode lasers are master oscillators (called the MOT and repump master) and are locked 
to the appropriate frequency through the saturation absorption spectroscopy laser frequency locking scheme discussed in Sec. 2.7.2 and Sec. 2.7.3. The remaining three diode lasers are injection locked by seeding them with light from the MOT master oscillator. The resultant near-resonance light is shifted in frequency with acousto-optic modulators to fine tune them to the precise frequency needed for each application.

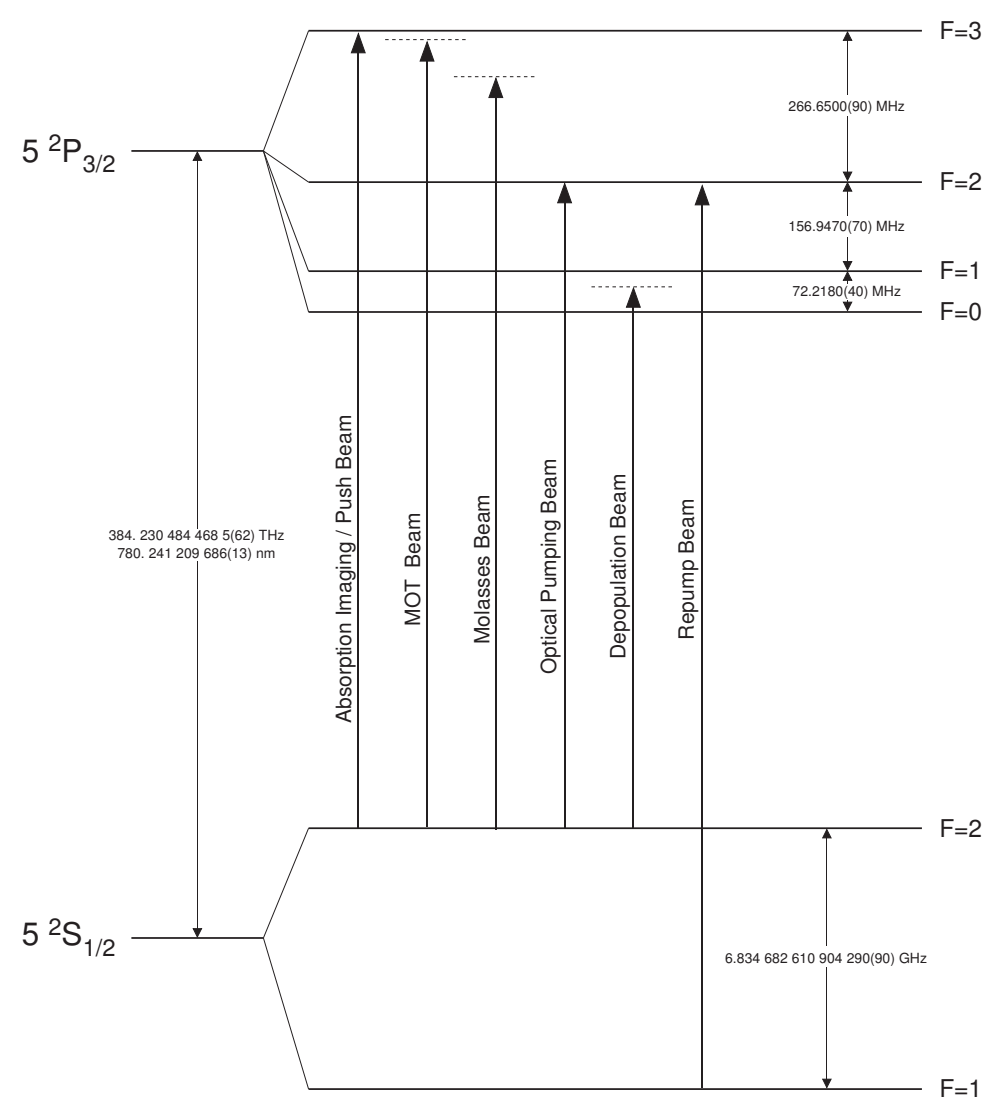

Figure 3.6: Near-resonance laser frequencies used in the experiment in relation to the hyperfine structure of the ${ }^{87} \mathrm{Rb} D_{2}$ transition. 


\subsubsection{MOT Master Laser}

The MOT master laser is based around a simple, inexpensive singlemode laser diode. The diode used in the experiment was purchased from Intelite Inc. (model: MLD-780-100S5P). This model operates nominally at a wavelength of $780 \mathrm{~nm}$ and output power of $100 \mathrm{~mW}$ with an injection current of $120 \mathrm{~mA}$.

The diode is made to lase at a chosen frequency and with a narrow bandwidth by placing it in a Littrow configured, grating-stabilized cavity [91, 92]. The cavity is shown in Fig. 3.7 and is formed from the reflective back facet of the laser diode and a grating. The diode is positioned in the cavity by first placing it in a collimation tube (Thorlabs, LT230P-B) which bundles its output into a low divergence beam. This is then inserted into a bronze holder which slides into a bronze mounting block, directing the optical radiation towards the grating. The grating (Edmund Scientific) is formed from a $500 \AA$ thick gold coating with 1200 grooves/mm etched into it. The grating face measures $12.5 \times 12.5 \mathrm{~mm}$. In the Littrow configuration the grating serves as both a cavity end mirror and an output coupler. The $1^{\text {st }}$ order reflection off of the grating is directed back into the laser diode, while the $0^{\text {th }}$ order reflection is used as the output beam. For the $1^{\text {st }}$ order beam to reflect back into the laser diode, the angle of incidence of the incoming beam with respect to the grating surface must satisfy the Littrow condition

$$
m \lambda=2 d \sin (\alpha)
$$




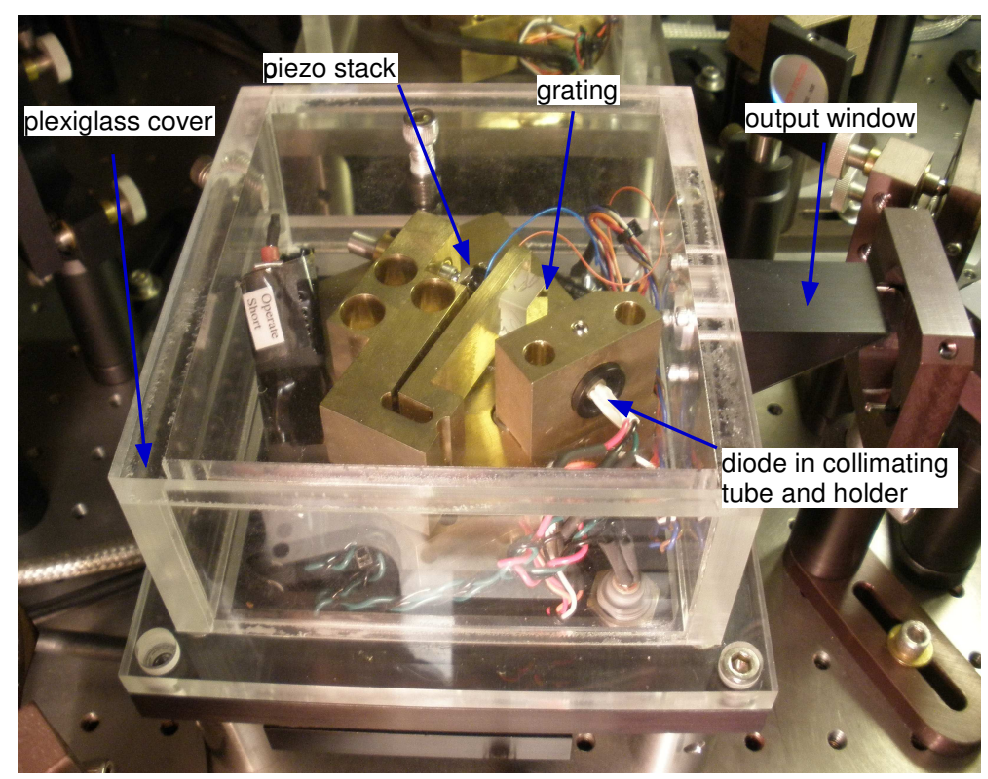

Figure 3.7: The MOT master laser is a simple laser diode placed in a Littrow configured cavity. The laser cavity is placed within a Plexiglass cover to help isolate it thermally from the environment.

where $m$ is the order of the reflection, $\lambda$ is the wavelength of light, $d$ is the groove spacing, and $\alpha$ is the angle of incidence. For a first-order reflection of a beam at $780 \mathrm{~nm}$ off of a grating with a line spacing of $d=$ $(1200 \text { grooves } / \mathrm{mm})^{-1}=0.833 \mu \mathrm{m}$, the incident angle must be $27.9^{\circ}$ for the beam to return to the laser diode. The lasing wavelength can be tuned in this configuration by slightly adjusting the angle of the grating which in turn slightly changes the cavity length and therefore the supported mode frequency.

For stable operation both the temperature and laser diode injection current must be regulated as they both affect the lasing frequency. The temperature of the laser diode is monitored with a $50 \mathrm{k} \Omega$ glass-bead thermistor 
(Fenwal Electronics) positioned beneath the bronze mounting block. The resistance of this bead (which is of course temperature dependent) is used in a feedback loop to lock the temperature. The temperature control unit was designed by Leo Hollberg's group at NIST in Boulder, CO and regulates the power to a thermo-electric cooler (Melcor, CP1.0-127-05L) positioned below the mounting block in response to the measured temperature. The laser diode injection current is regulated by a PID circuit designed and built by a previous student [84].

The angle of the grating can be adjusted by hand with two fine-pitched screws. Behind one of the screws is a stack of three piezo discs (American Piezo Ceramics, $8 \mathrm{~mm} \times 2.54 \mathrm{~mm} ; 1.35 \mu \mathrm{m} / \mathrm{kV}$ ) which is used to fine-tune the angle electronically. Feedback on the peizo stack allows us to lock the frequency of the MOT master laser.

Our locking scheme is accomplished by picking off a small portion $(\sim$ $1 \mathrm{~mW}$ ) of the output of the MOT master laser with a $103 \mathrm{MHz} \mathrm{AOM}$ and sending it to a saturation absorption spectroscopy setup. This setup yields the Doppler-free spectrum of the $F=2 \rightarrow F^{\prime}$ transitions as well as the crossover resonances. This spectrum is converted into a suitable error signal for use in a PID loop.

The saturation absorption spectroscopy setup used in this experiment to lock the MOT master laser frequency is shown in Fig. 3.8. The pickedoff portion of the MOT master laser beam sent to the saturation absorption spectroscopy setup is split unequally into a strong pump beam and a weak 


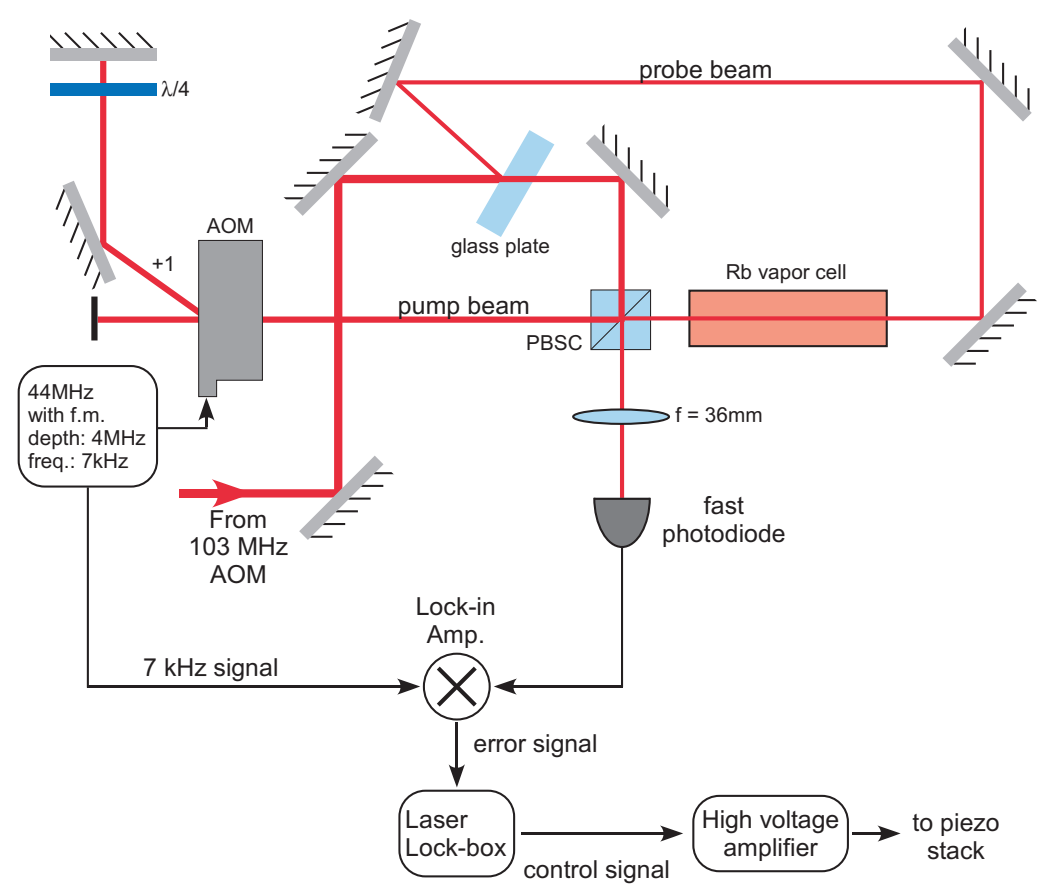

Figure 3.8: Layout of the MOT master saturation absorption spectroscopy setup used to lock this laser to the correct frequency.

probe beam by a glass plate. The probe beam is directed by a series of mirrors around the $\mathrm{Rb}$ vapor cell and then through it. After passing through the vapor cell the probe beam is directed by a polarizing beam splitter cube (PBSC) into a lens $(\mathrm{f}=36 \mathrm{~mm})$ focusing the beam onto a fast photodiode. The pump beam is reflected by a PBSC directing it into an AOM. The AOM is driven by a frequency modulated (f.m.) signal centered at $44 \mathrm{MHz}$. The modulation depth and frequency are $4 \mathrm{MHz}$ and $7 \mathrm{kHz}$ respectively. The purpose of the frequency modulation will be clear shortly. The $1^{\text {st }}$ order diffracted beam from the AOM passes through a $\lambda / 4$ waveplate and is then retro-reflected by a spherical mirror 
back into the AOM in a standard double pass configuration. The $1^{\text {st }}$ order spot from the double passed beam passes through the PBSC once again, but because it has passed through a $\lambda / 4$ waveplate twice its polarization has rotated $90^{\circ}$ and so is transmitted by the cube. The pump beam then passes though the $\mathrm{Rb}$ vapor cell, overlapping the probe beam.

Because the probe beam has double passed a $44 \mathrm{MHz}$ AOM (each time the $1^{\text {st }}$ order beam was used) its frequency has been shifted up by $88 \mathrm{MHz}$. This results in the pump and probe beam interacting with atoms which have velocity such that they are Doppler shifted by $44 \mathrm{MHz}$ and not with the stationary atoms as discussed in Sec. 2.7.2. The result is that the signal produced by the fast photodiode reflects the Doppler-broadened transition with peaks at each of the real transitions $\left(F=2 \rightarrow F^{\prime}=1,2,3\right)$ as well as the cross-over resonances $(F=2 \rightarrow 1 / 2,1 / 3,2 / 3)$ shifted by $44 \mathrm{MHz}$ from their zero velocity value. This signal is mixed in a lock-in amplifier (SRS SR510) with the f.m. modulation frequency, resulting in a Doppler-free dispersion signal (see Sec. 2.7.2).

Any of the dispersive curves, shown in Fig. 3.9, could be used as an error signal to lock the master laser frequency, but we used the $F=2 \rightarrow 2 / 3$ cross-over transition because it is the most prominent. This signal serves as an error signal and is sent to a home-built PID [89] lockbox, which outputs a control signal which we amplify with a Trek 601B-2 high voltage amplifier. The amplified signal drives the piezo stack, controlling the lasing frequency and closing the loop.

While this setup allows us to lock the MOT master laser, the laser out- 


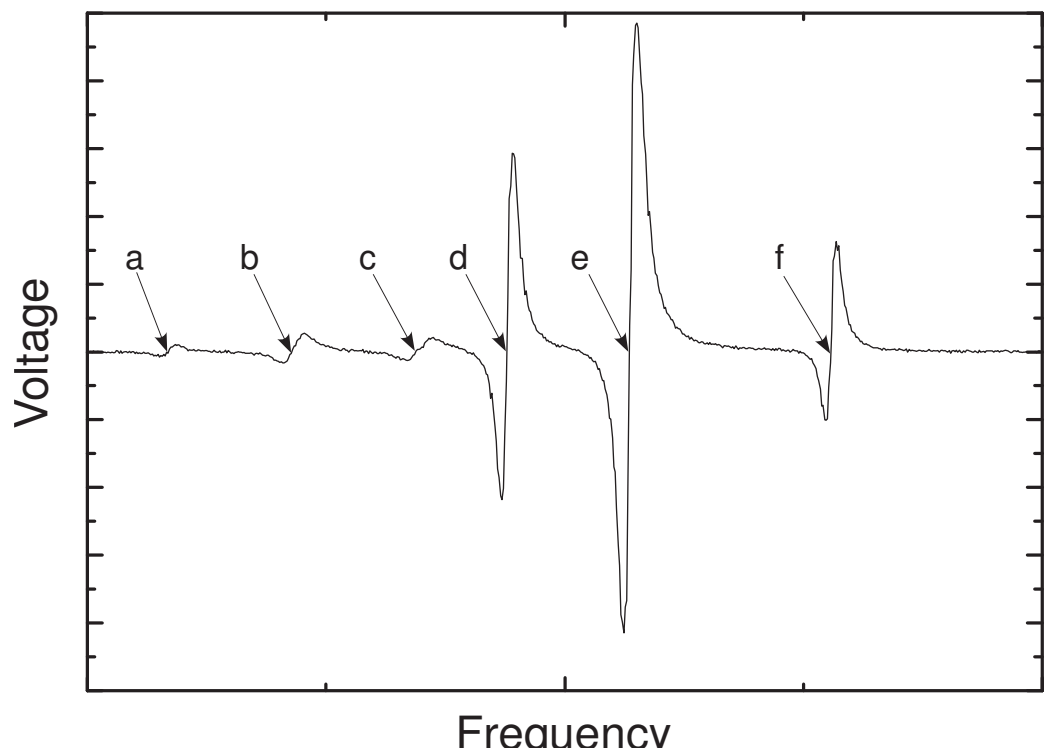

Figure 3.9: The MOT saturated absorption spectroscopy dispersive signal. The real and cross-over transitions corresponding to each dispersive lineshape are as follows: a) $F=2 \rightarrow F^{\prime}=1$ b) $F=2 \rightarrow F^{\prime}=1 / 2$ c) $F=2 \rightarrow F^{\prime}=2$ d) $F=2 \rightarrow F^{\prime}=1 / 3$ e) $F=2 \rightarrow F^{\prime}=2 / 3$ f) $F=2 \rightarrow F^{\prime}=3$.

put is not at the $F=2 \rightarrow F^{\prime}=3$ transition frequency, which is needed for the operation of the MOT. The AOM which initially picked off the small portion of the beam sent to the saturation absorption spectroscopy setup shifted the beam up by $103 \mathrm{MHz}$. Because the probe beam was shifted up by $88 \mathrm{MHz}$ the spectrum obtained corresponded to atoms shifted by $44 \mathrm{MHz}$ so the MOT master laser output is therefore shifted $147 \mathrm{MHz}$ to the red of the $F=2 \rightarrow 2 / 3$ cross-over resonance transition frequency. The $F=2 \rightarrow 2 / 3$ cross-over resonance transition frequency is $133 \mathrm{MHz}$ to the red of the $F=2 \rightarrow F^{\prime}=3$ transition so the MOT master output is $280 \mathrm{MHz}$ to the red of this transition. 
This frequency offset is compensated for in the experimental setup by a method allowing us to tune the frequency output of the MOT master laser over the range needed to operate most of the near-resonance laser tasks. How this is done is shown in Fig. 3.10 which displays the distribution of the MOT master beam in the experiment setup. Most of the MOT master laser output power passes through the $103 \mathrm{MHz}$ AOM unshifted. This portion of the beam

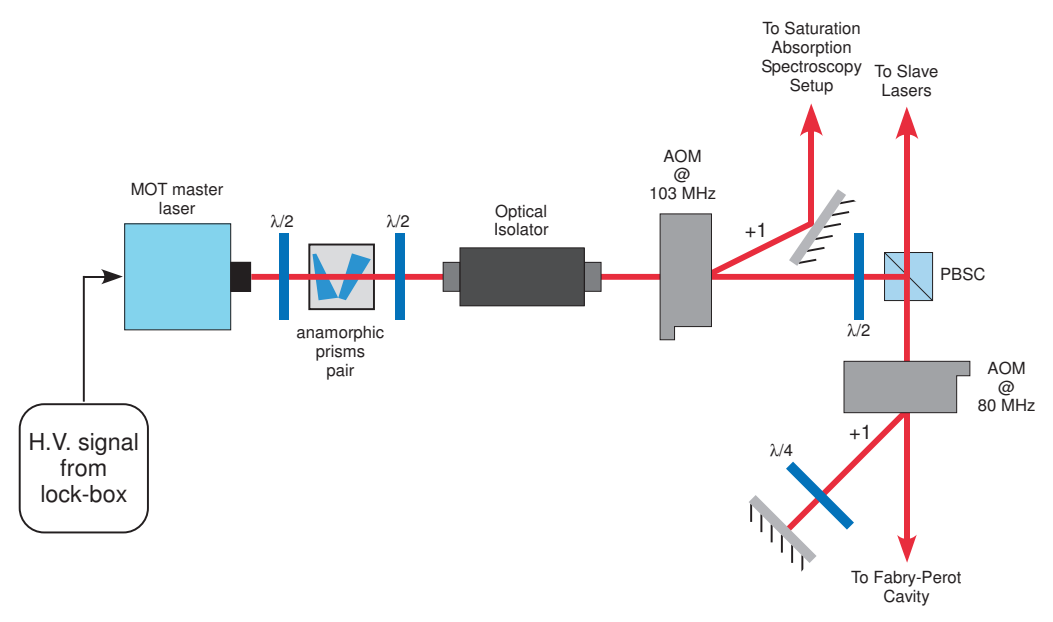

Figure 3.10: Distribution of the MOT master laser output. A portion of the beam is sent to the saturation absorption spectroscopy setup to lock the laser. Of the remaining portion, a small amount is sent to a Fabry-Perot cavity to monitor the spectrum. The rest is used to injection lock the slave lasers.

double passes an AOM driven at a frequency of $80 \pm 20 \mathrm{MHz}$. This shifts the beam to somewhere between $80-160 \mathrm{MHz}$ to the red of the $F=2 \rightarrow F^{\prime}=3$ transition. We then use $80 \mathrm{MHz}$ AOMs as fast beam shutters elsewhere in the experiment so that the beams derived from the MOT master laser have frequency detunings $0-80 \mathrm{MHz}$ to the red of the $F=2 \rightarrow F^{\prime}=3$ transition. This range covers the spectrum needed for a large portion of the near-resonance 
beams. For example the MOT and optical molasses beams are tuned $15 \mathrm{MHz}$ and $50 \mathrm{MHz}$ to the red of the $F=2 \rightarrow F^{\prime}=3$ transition, respectively.

\subsubsection{Slave Lasers}

The MOT master laser does not produce enough optical power to operate the upper and lower MOT. The frequency stabilization process described in the previous section reduces the output power of the laser diode used in the MOT master laser from its free running level $(\sim 100 \mathrm{~mW})$ to roughly $\sim 30 \mathrm{~mW}$. One method of increasing the available power at a particular frequency is to use "slave lasers" frequency locked to a master oscillator. Our experimental apparatus utilizes three slave lasers, each frequency locked to the MOT master laser output. Once locked, the result is three diode lasers with the same spectral properties as the MOT master laser outputting an optical power near their free-running level.

Similar to the MOT master laser, the slave lasers are designed around a very inexpensive laser diode. The diode used in each slave laser (Digi-Key: GH0781JA2C) outputs a single-mode near the nominal operating wavelength of $784 \mathrm{~nm}$. Even though the nominal output wavelength is $784 \mathrm{~nm}$, it can be tuned with temperature to the needed value of $780 \mathrm{~nm}$. The maximum freerunning optical power of these laser diodes is $120 \mathrm{~mW}$ operating at an injection current of $167 \mathrm{~mA}$.

Like the MOT master laser diode, each slave laser diode is placed in a collimation tube (Thorlabs: LT230P-B) which is inserted into a bronze holder. 
The holder is placed into a bronze mounting block. Below the mounting block is a TEC, which is used to control the temperature of the laser diode in conjunction with a thermistor and temperature controller, see Fig. 3.11. The injection current into each diode is controlled with a PID loop identical to
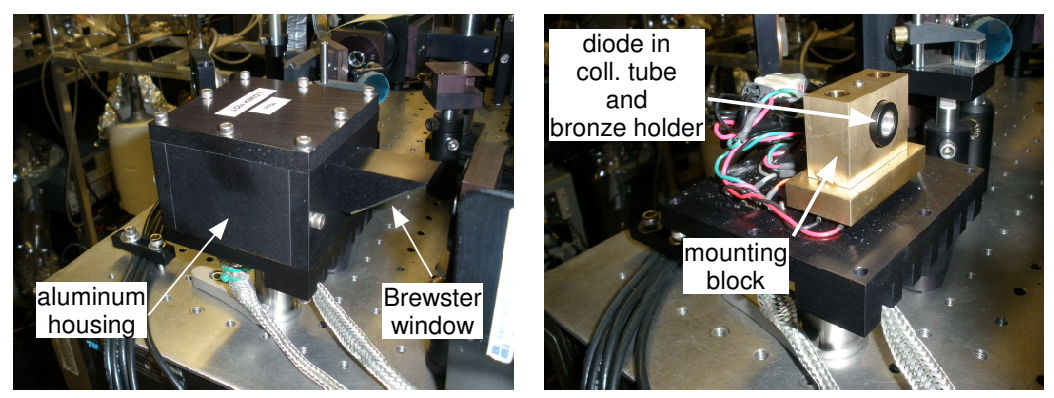

Figure 3.11: A slave laser used in the experiment to generate sufficient resonant light. The left image shows the laser with its aluminum housing in place. The right image is taken without the aluminum housing, revealing the laser diode and mounting block.

that used in the MOT master laser.

Injection locking is accomplished by seeding the free-running diode lasers with a weak beam spatially matched to the diode laser output. We seed a small amount of light $(\sim 2 \mathrm{~mW})$ from the MOT master laser beam into each slave laser. This is done by using the optical isolators (ConOptics: 713B) placed after each slave laser. The seed light is sent into the rejection port of each optical isolator collinear to the rejected portion of the beam from the slave lasers as shown in Fig. 3.12. Because optical isolators utilize Faraday rotation to work, they rotate the polarization of light passing though them in the same direction regardless of the direction of travel. Therefore the seed 


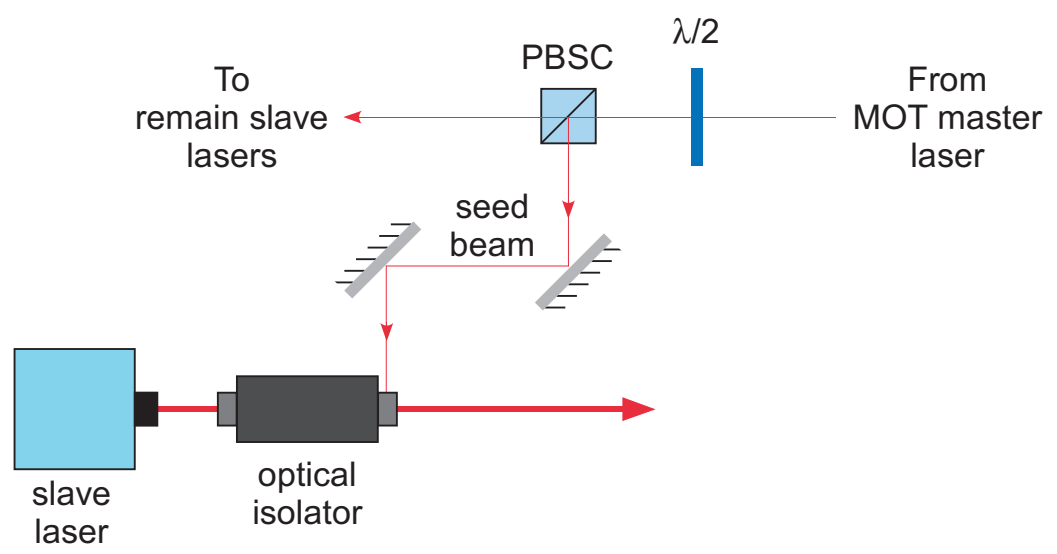

Figure 3.12: The slave lasers are injected using the rejection port of the optical isolator protecting each laser from back reflections.

beam emerges from the optical isolator with the same polarization as the slave laser output. A more detailed description of the seeding procedure can be found in reference [83].

Injection locking can be understood by considering free-running laser diodes to be regenerative amplifiers with a natural oscillation frequency $\omega_{0}$ and output power $I_{0}$. If a beam of intensity $I_{1}$ is seeded into this amplifier, its intensity at the output will be $\left|\tilde{g}\left(\omega_{1}\right)\right|^{2} I_{1}$, where $\tilde{g}\left(\omega_{1}\right)$ is the frequency dependent gain of the laser medium. If $\omega_{0}$ (which we control with temperature and the injection current) is close to $\omega_{1}$ then the value of $\left|\tilde{g}\left(\omega_{1}\right)\right|^{2} I_{1}$ approaches $I_{0}$. In this limit the amplified signal begins to steal enough gain from the lasing medium that the free-running laser oscillation dies out. Much more detail on this process can be found in [93].

The distribution of the three slave lasers is discussed in the next several 
sections.

\subsubsection{Upper MOT Horizontal Slave Laser}

As suggested by the name, the primary function of this slave laser is to provide optical power to the upper MOT horizontal beams. This laser produces more than enough power for this purpose, so it is used to create additional beams. 


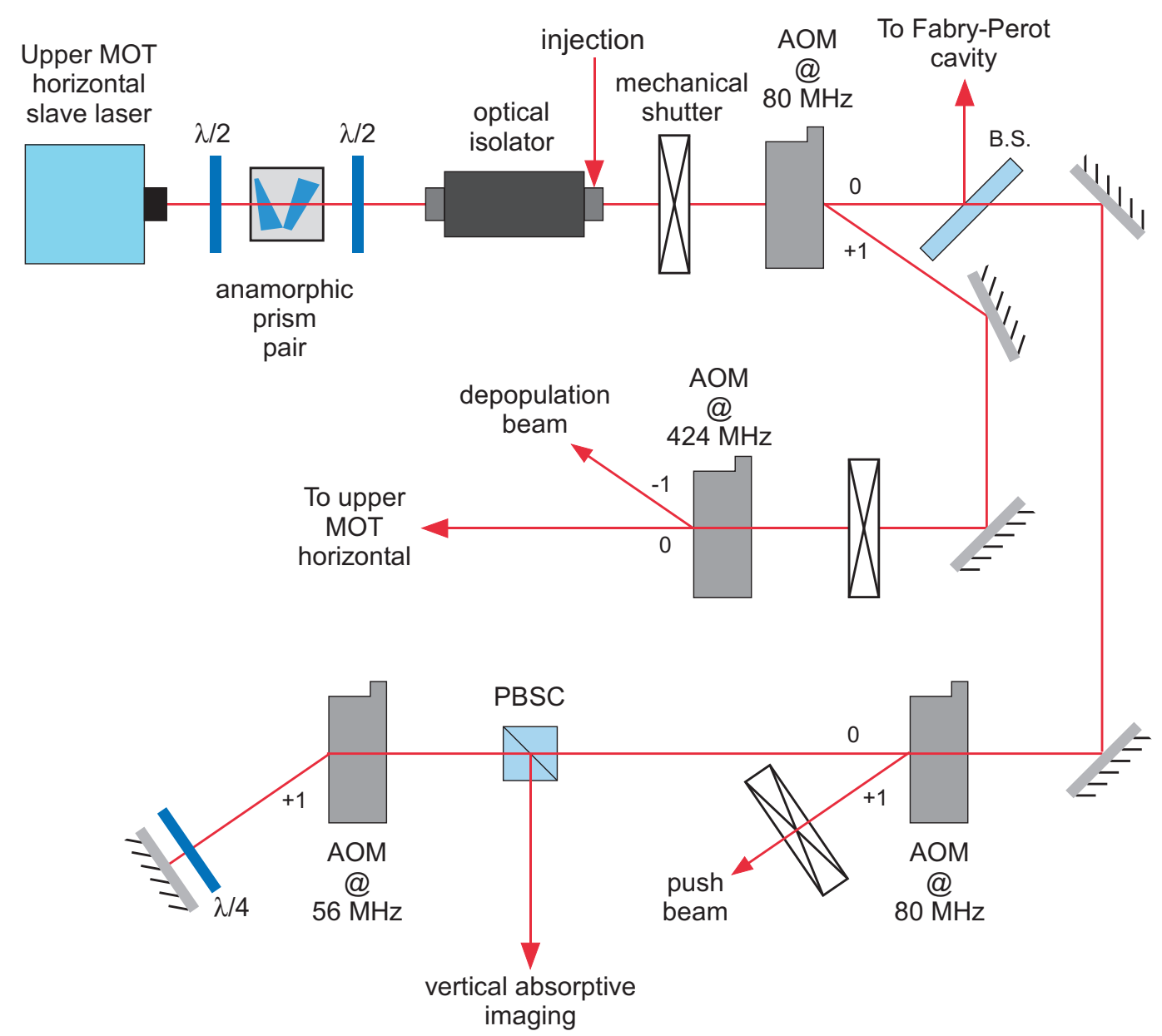

Figure 3.13: Upper MOT Horizontal Slave Laser beam distribution.

These include the vertical absorptive imaging beam, the push beam, and the depopulation beam. The distribution of power from this laser is illustrated in Fig. 3.13. After the beam exits the laser housing it passes through an anamorphic prism pair to remove its astigmatism. Then it passes through an optical isolator protecting the laser diode from back reflections. The beam 
then passes through an $\mathrm{AOM}$ driven at $80 \mathrm{MHz}$. A portion of the $0^{\text {th }}$ order beam is sent to a Fabry-Perot interferometer to monitor its spectrum. The remaining portion of the $0^{\text {th }}$ order beam passes through a second AOM driven at $80 \mathrm{MHz}$. The first order spot from this AOM is shifted into resonance with the $|F=2\rangle \rightarrow\left|F^{\prime}=3\right\rangle$ transition (with the correct injection frequency) and is used as the push beam. This AOM is used as a fast shutter to block the beam when not wanted. We do this by blocking the drive signal with an rf voltage controlled attenuator. However even with full attenuation some light is present in the first order. To completely block this light a slower $(\sim 2 \mathrm{~ms})$ mechanical shutter is used. The zeroth order beam from the second $80 \mathrm{MHz}$ AOM double passes a $56 \mathrm{MHz} \mathrm{AOM}$ and is used for vertical absorptive imaging. The first order beam from the first $80 \mathrm{MHz}$ AOM passes through an AOM driven at $424 \mathrm{MHz}$. The zeroth order beam from this AOM is used as the upper MOT horizontal beams. The $-1^{\text {st }}$ order beam is used as the depopulation beam in the single-photon cooling process. More details on this beam can be found in Sec. 3.2.1.7.

\subsubsection{Upper MOT Diagonal Slave Laser}

This slave laser is used primarily to produce the upper MOT diagonal beams. The excess power is used to create the optical pumping beam and the horizontal absorptive imaging beam. The distribution of power from this laser is illustrated in Fig. 3.14. This beam also starts by passing through an anamorphic prism pair and optical isolator. After that it passes through an 


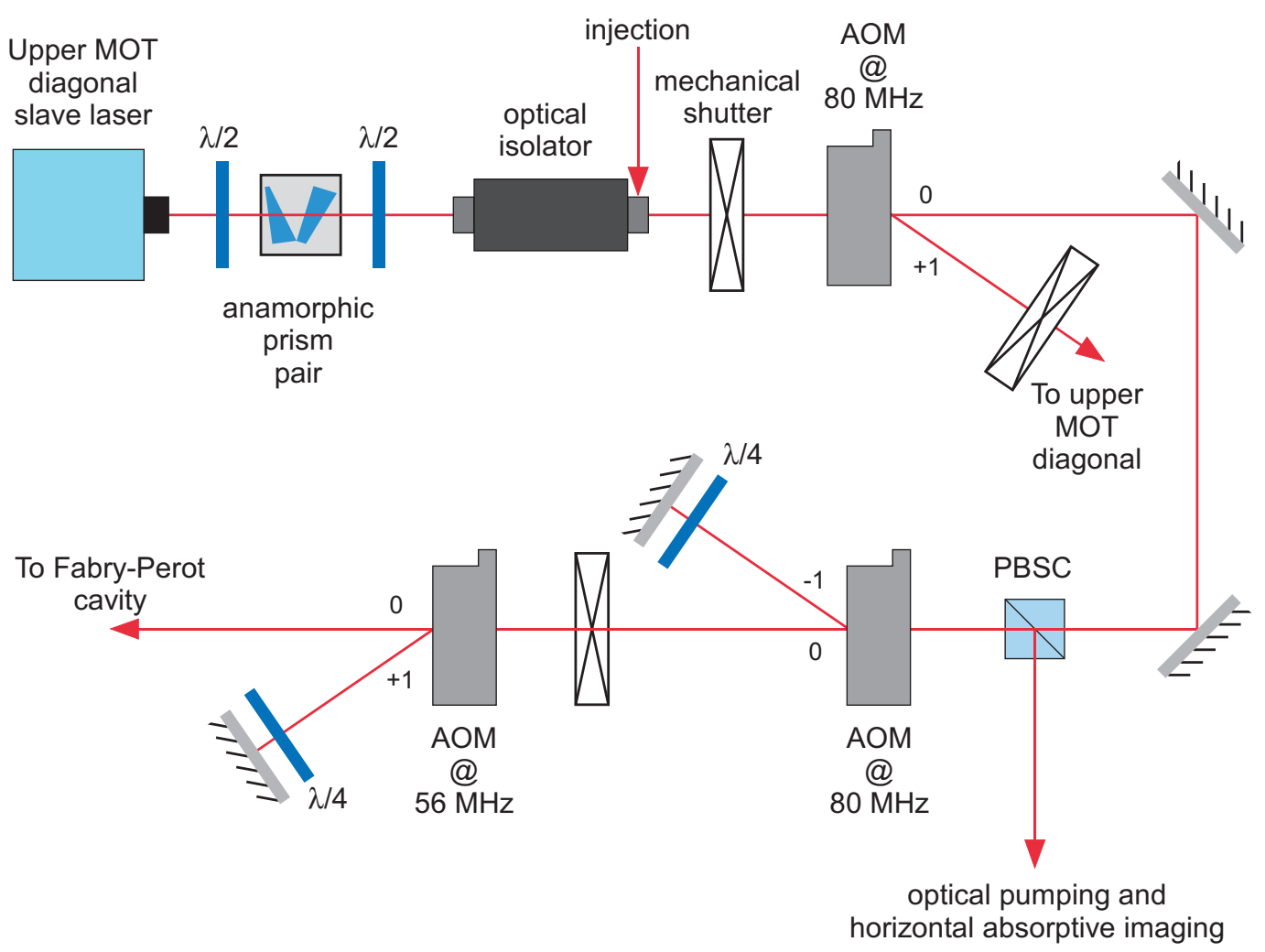

Figure 3.14: Upper MOT Diagonal Slave Laser beam distribution.

AOM driven at $80 \mathrm{MHz}$. The first order beam is used to create the upper MOT diagonal beams. This AOM serves as a fast but imperfect shutter which is augmented by a slow mechanical shutter. The $0^{\text {th }}$ order beam then double passes an $80 \mathrm{MHz} \mathrm{AOM}$ which downshifts the frequency into resonance with the $|F=2\rangle \rightarrow\left|F^{\prime}=2\right\rangle$ transition, and is used to optically pump the atoms into the $\left|F=2, m_{F}=2\right\rangle$ state. The zeroth order beam which passes through the second $80 \mathrm{MHz} \mathrm{AOM}$ is double passed through an $\mathrm{AOM}$ driven at $56 \mathrm{MHz}$ to bring it into resonance with the $|F=2\rangle \rightarrow\left|F^{\prime}=3\right\rangle$ transition. This beam 
is used as the horizontal absorptive imaging beam. The $0^{\text {th }}$ order beam from the $\mathrm{AOM}$ driven at $56 \mathrm{MHz}$ is sent to a Fabry-Perot interferometer to monitor its spectrum.

\subsubsection{Lower MOT Slave Laser}

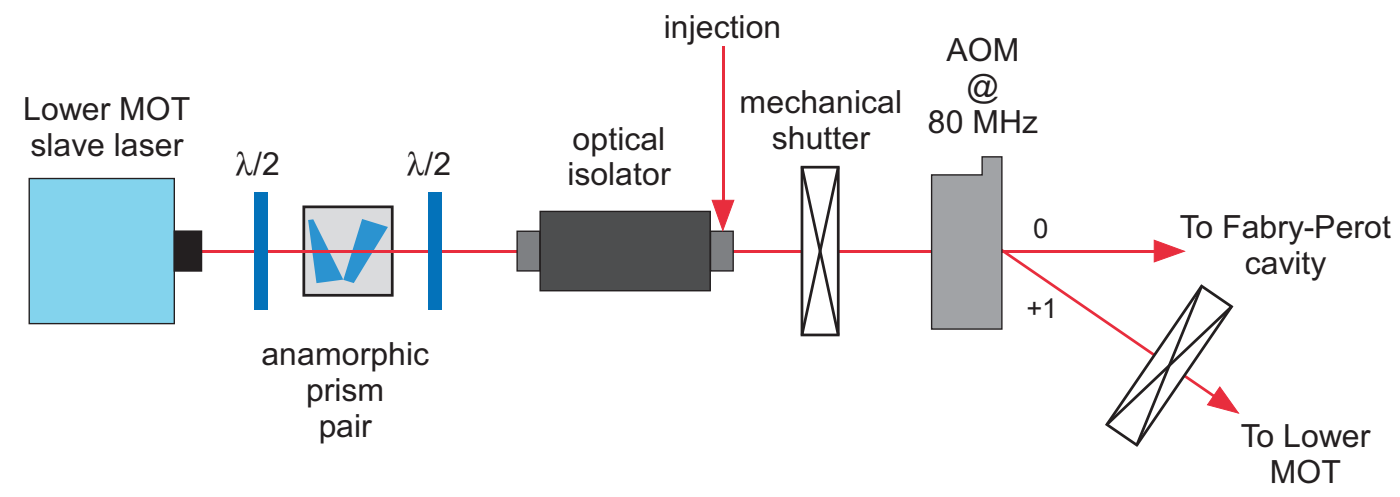

Figure 3.15: Lower MOT Slave Laser beam distribution.

This slave laser is used for all six lower MOT beams and has the simplest distribution scheme (Fig. 3.15). After passing through an anamorphic prism pair and optical isolator this beam is diffracted by an AOM driven at $80 \mathrm{MHz}$. The first order beam is in resonance with the $|F=2\rangle \rightarrow\left|F^{\prime}=3\right\rangle$ transition (with the correct injection frequency). The $0^{\text {th }}$ order component is sent to a Fabry-Perot to monitor its spectrum.

\subsubsection{Repump Master Laser}

As suggested by the name, the repump master laser is used to provide optical power to the upper and lower repump beams. Repump beams are 
needed when forming a MOT or optical molasses because ${ }^{87} \mathrm{Rb}$ atoms can decay into the $|F=1\rangle$ ground state manifold where they are no longer resonant with the MOT beams, removing them from the cooling cycle. The repump beam is resonant with the $|F=1\rangle \rightarrow\left|F^{\prime}=2\right\rangle$ transition and reintroduces atoms into the $|F=2\rangle \rightarrow\left|F^{\prime}=3\right\rangle$ cycling transition.

The construction of the repump master laser is identical to that of the MOT master laser and so the details will not be repeated here. The temperature and injection current are also controlled in a manner identical to that used for the MOT master laser.

As with the MOT master laser, feedback on a piezo stack positioned behind the grating allows us to lock the frequency of the repump master laser. In this case we lock the laser to the $F=1 \rightarrow 1 / 2$ cross-over resonance frequency. This locking scheme is accomplished by picking off a small portion $(\sim 1 \mathrm{~mW})$ of the output of the repump master laser with a polarizing beam splitting cube (PBSC) and sending it to a saturation absorption spectroscopy setup. This setup yields the Doppler-free spectrum of the $|F=1\rangle \rightarrow\left|F^{\prime}\right\rangle$ transitions as well as the cross-over resonances.

The saturation absorption spectroscopy setup used to lock the repump master laser differs from that used to lock the MOT master laser. The layout of this scheme is shown in Fig. 3.16. The portion of the repump master laser sent to the saturation absorption spectroscopy setup encounters a thick glass plate which splits it into three beams: a strong pump beam, a weak probe beam and a weak reference beam. The probe and reference beam are displaced from one 


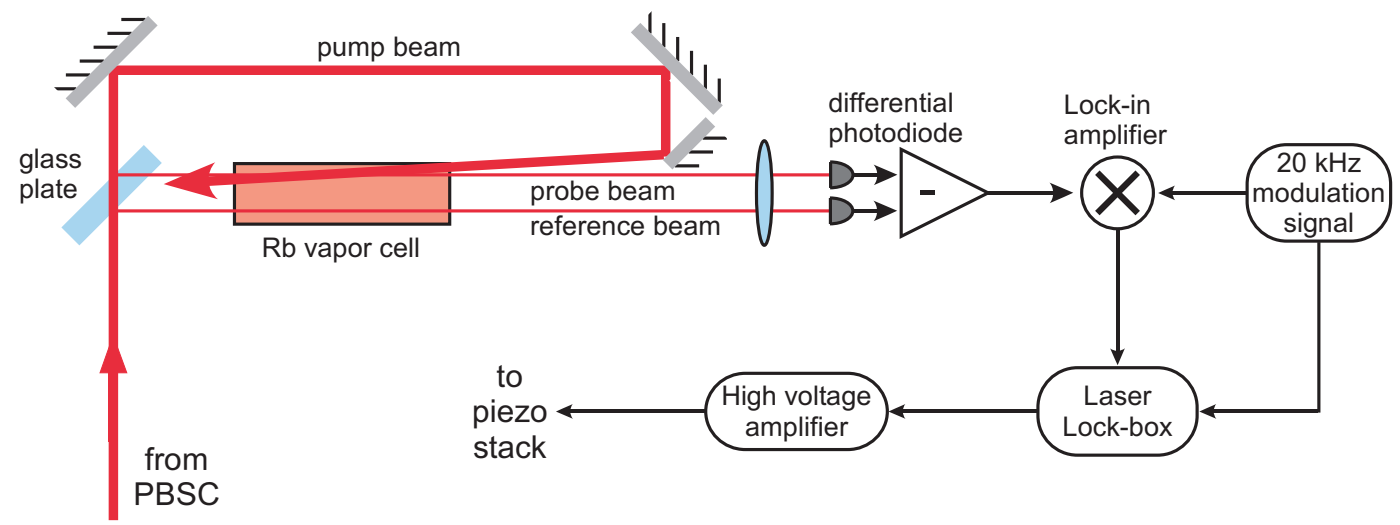

Figure 3.16: Layout of the saturation absorption spectroscopy locking scheme used to control the frequency of the repump master laser.

another and parallel. Both beams are directed through a $\mathrm{Rb}$ vapor cell. The pump beam travels around the Rb vapor cell and then through it, overlapping the probe but not the reference beam. The probe and reference beams are each focused onto a fast photodiode, and the difference between the signals is monitored.

The reason the differential photodiode is used becomes clear by considering the intensity of the probe and reference beams as the frequency of the laser is slowly swept through resonance. The intensity of the reference beam will reflect the Doppler-broadened transition. The probe beam intensity will also have a Doppler-broadened background but will spike at transitions and cross-over resonances. By taking the difference of these signals we are able to extract the Doppler-free spectrum.

To obtain a signal which can be locked to, the grating in the repump 
master laser is dithered at $20 \mathrm{kHz}$. The signal from the differential photodiode is mixed with the dithering frequency in a lock-in amplifier, resulting in a dispersion signal, shown in Fig. 3.17. We lock to the dispersion curve corresponding to the $F=1 \rightarrow 1 / 2$ cross-over resonance because it is the most

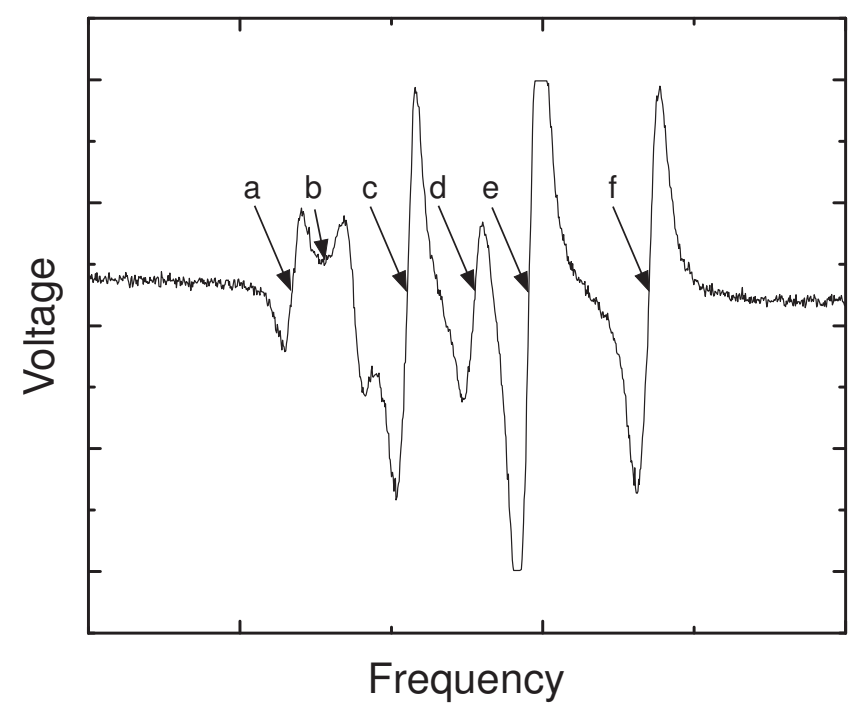

Figure 3.17: The dispersion curves correspond to the following transitions: a) $F=1 \rightarrow F^{\prime}=0$, b) $F=1 \rightarrow 0 / 1$, c) $F=1 \rightarrow F^{\prime}=1$, d) $F=1 \rightarrow 0 / 2$, e) $F=1 \rightarrow 1 / 2$, f) $F=1 \rightarrow F^{\prime}=2$.

prominent.

The hyperfine splitting of the $F^{\prime}=1$ and $F^{\prime}=2$ state is roughly $157 \mathrm{MHz}$, so the repump master laser frequency is locked $78.5 \mathrm{MHz}$ below the desired $|F=1\rangle \rightarrow\left|F^{\prime}=2\right\rangle$ transition frequency. This beam is shifted to the correct frequency with an $80 \mathrm{MHz}$ AOM before being split by a $\mathrm{PBSC}$ and sent to the upper and lower MOT as shown in Fig. 3.18. 


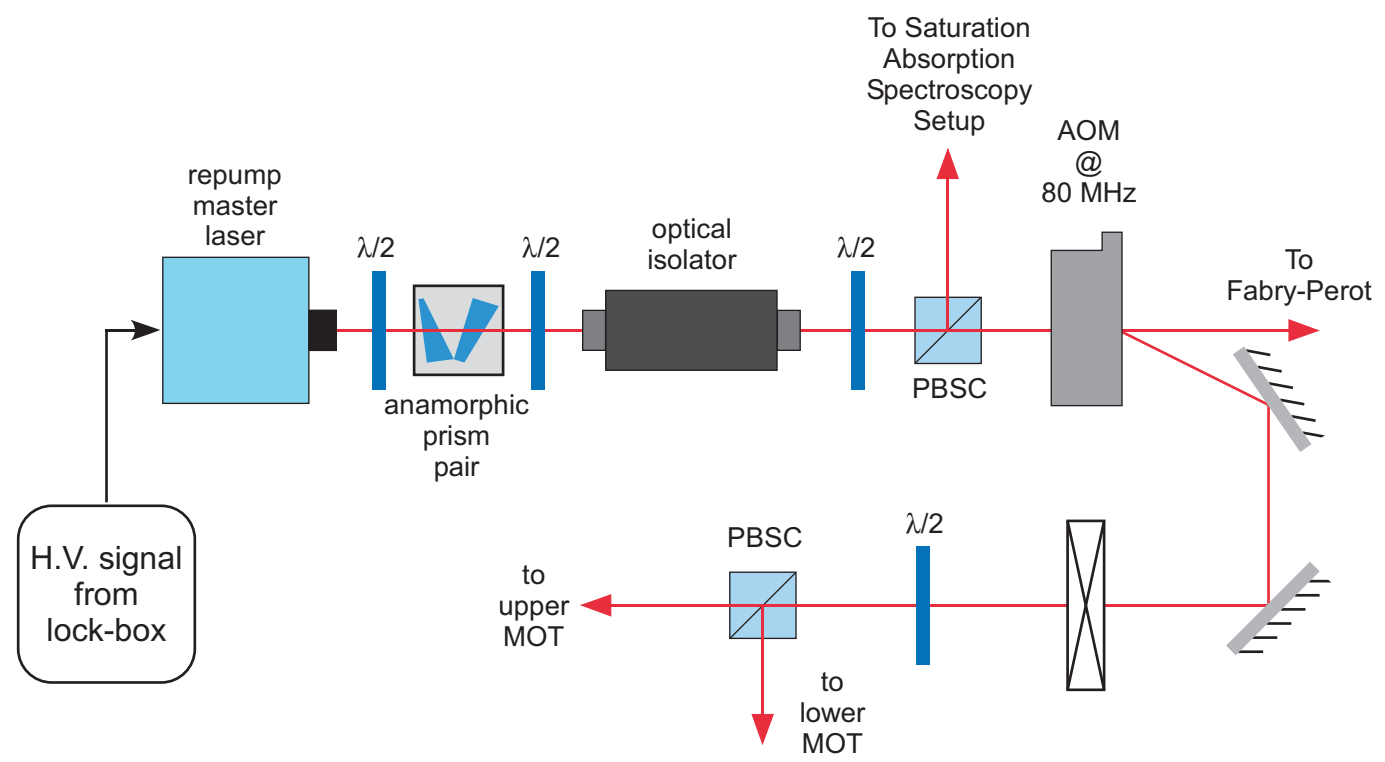

Figure 3.18: Distribution of the repump master laser beam.

\subsubsection{Depopulation Beam}

The depopulation beam is used during the single-photon cooling process to transfer atoms from the magnetically trappable $\left|F=2, m_{F}=2\right\rangle$ state to the magnetically decoupled $\left|F=1, m_{F}=0\right\rangle$ state. More detail on its use during this process can be found in Ch. 4. This section describes the construction of this beam.

The depopulation beam is derived from the upper horizontal MOT beam as shown in Fig. 3.19. The upper horizontal MOT beam passes through an AOM driven at $424 \mathrm{MHz}$ and the $-1^{\text {st }}$ order beam is used as the depopulation beam. After passing through the AOM, the beam is resonant with the $|F=1\rangle \rightarrow\left|F^{\prime}=2\right\rangle$ transition frequency. The depopulation beam is coupled 


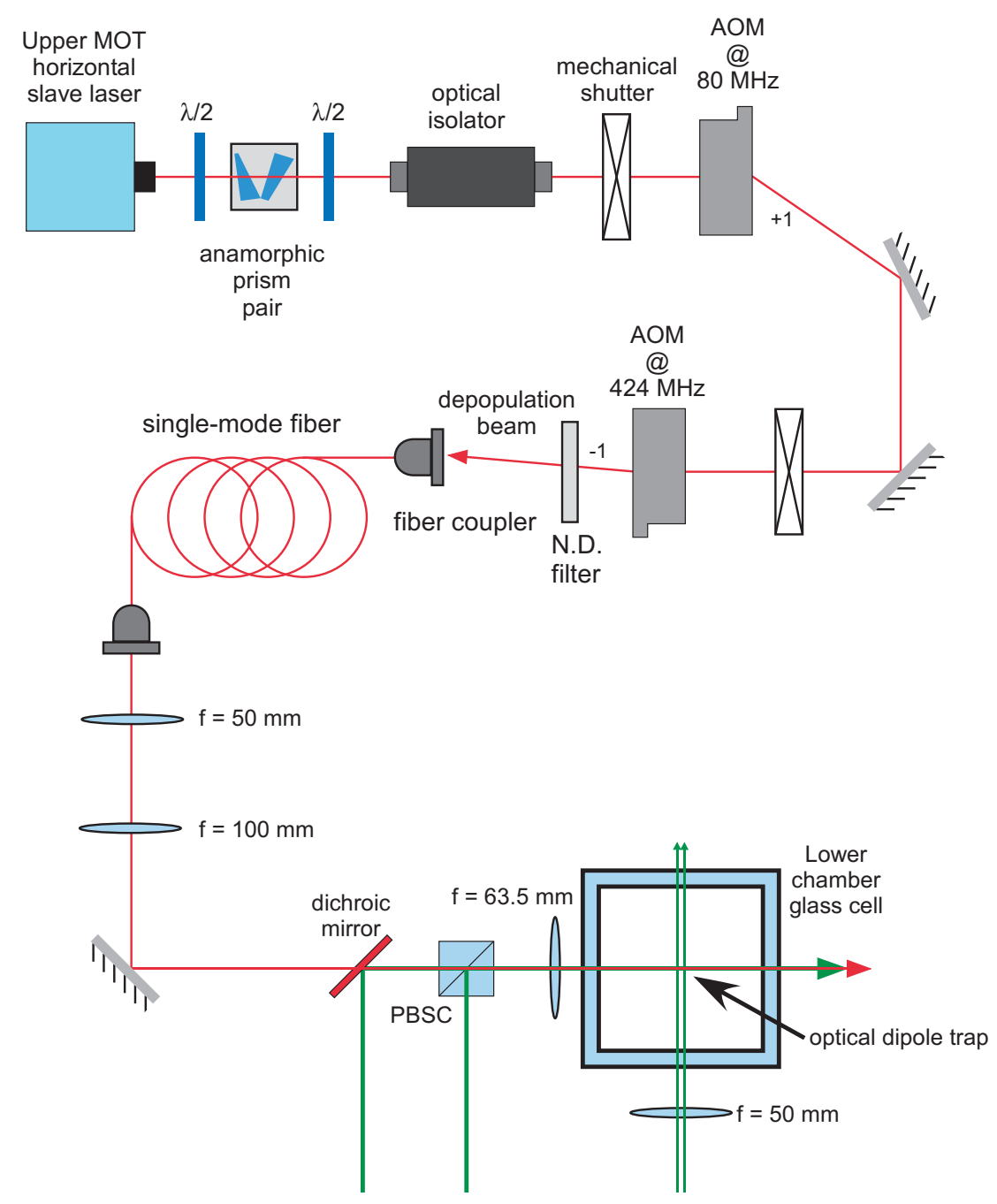

Figure 3.19: The depopulation beam is derived from the upper horizontal MOT beam. An AOM driven at $424 \mathrm{MHz}$ is used to downshift the frequency of the MOT beam to be resonant with the $|F=2\rangle \rightarrow\left|F^{\prime}=1\right\rangle$ transition.

into a single-mode optical fiber and routed to a magnifying telescope comprised of a $50 \mathrm{~mm}$ and $100 \mathrm{~mm}$ lens. The nominal spacing between these two 
lenses is $150 \mathrm{~mm}$, however the spacing is adjustable, allowing us to control the beam curvature and hence the location of the beam waist after it is focused by a $63.5 \mathrm{~mm}$ lens into the lower chamber glass cell. The power needed in the depopulation beam is very small, typically around a few nanowatts.

\subsubsection{Far-Detuned Laser}

We use far-detuned laser beams to create optical dipole traps to confine atoms. A discussion of the theory used to estimate the depth of these traps is given in Sec. 2.5.1. There it was seen that in the limit of negligible saturation and a frequency detuning much larger than the fine structure splitting, the optical dipole potential is given by

$$
U_{d i p}(\vec{r})=\frac{3 \pi c^{2}}{2 \omega_{0}^{3}} \frac{\Gamma}{\Delta} I(\vec{r}) .
$$

The laser used to create the optical dipole traps, the Verdi V10, is a commercially available turn-key system manufactured by Coherent, Inc. This laser outputs a single-mode (both longitudinally and transversely) with up to $10 \mathrm{~W}$ of power at $532 \mathrm{~nm}$. The linewidth is specified to be $<5 \mathrm{MHz}$ and was measured over $50 \mathrm{~ms}$ with a thermally stabilized reference etalon. The output beam diameter and divergence are specified to be $2.25 \mathrm{~mm}$ and $<0.5 \mu \mathrm{rad}$, respectively. The beam is vertically polarized and nearly Gaussian $\left(M^{2}<1.1\right)$. The pointing stability, power stability and RMS noise are specified as $<2 \mu \mathrm{rad}$ $/{ }^{\circ} \mathrm{C}, \pm 1 \%$ and $<0.03 \%$, respectively. These laser parameters are summarized in Table 3.1. 


\begin{tabular}{|l|c|}
\hline Output Power & $>10 \mathrm{~W}$ \\
\hline Wavelength & $532 \mathrm{~nm}$ \\
\hline Linewidth & $<5 \mathrm{MHz}$ \\
\hline Beam Diameter & $2.25 \pm 10 \% \mathrm{mrad}$ \\
\hline$M^{2}$ & $<1.1$ \\
\hline Power Stability & $\pm 1 \%$ \\
\hline Pointing Stability & $<2 \mu \mathrm{rad}$ \\
\hline Noise (RMS) & $<0.03 \%$ \\
\hline Polarization & Vertical, $>100: 1$ \\
\hline
\end{tabular}

Table 3.1: Verdi V10 system specifications.

The Verdi V10 outputs radiation at $532 \mathrm{~nm}$ which is far blue detuned from the ${ }^{87} \mathrm{Rb} D_{2}$ transition frequency. This means that $U_{d i p}(\vec{r})$ is positive everywhere and so a repulsive potential is formed. Our strategy to form 3D trapping potentials with repulsive barriers has been to use several "light sheets" to construct optical cups that hold the atoms against gravity. During the singlephoton cooling process atoms are transferred from the magnetic trap into an optical cup. During the course of the experiments we used two main geometries for the construction of the cup: an "optical box" and "optical trough." The process used to form these traps is very similar so I will only discuss the construction of the optical trough.

The optical trough comprises four Gaussian sheets as shown in Fig. 3.20. The sheets were formed by asymmetrically focusing the beam with cylindrical lenses. Two sheets travel along the $\hat{x}$ direction forming a "V"-shape. With the aid of gravity along the $-\hat{z}$ direction these sheets confined the atoms along the $\hat{z}$ and $\hat{y}$ directions. Two more vertically elongated sheets traveling along 
the $\hat{y}$ direction serve as "end caps" sealing the optical trap along the $\hat{x}$ direction. Each beam had approximately $0.7 \mathrm{~W}$ of optical power and together with gravity form a trap $\sim 10 \mu \mathrm{K}$ deep.

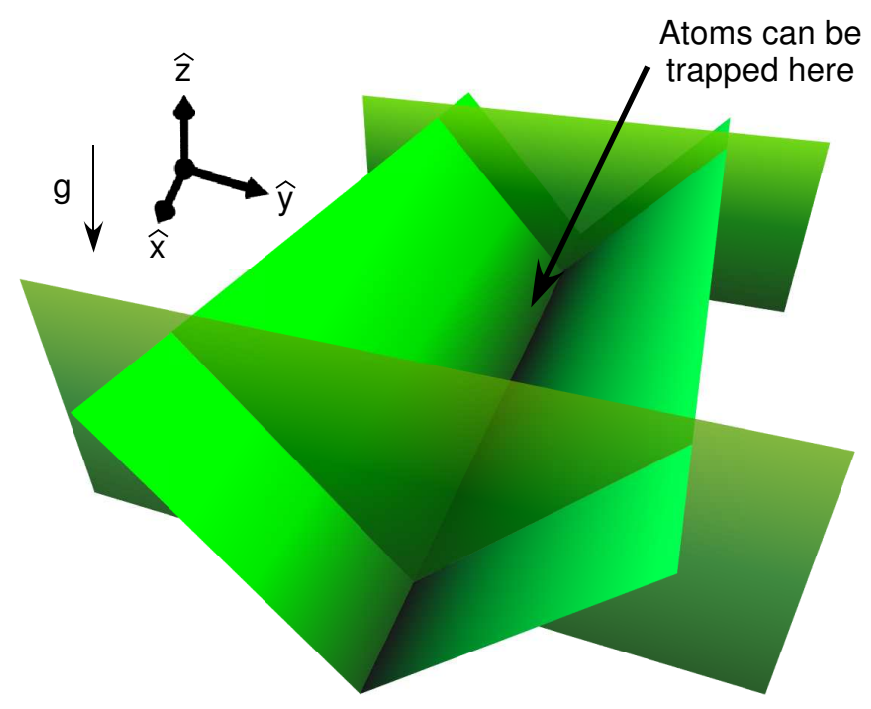

Figure 3.20: The optical trough used to catch atoms during the single-photon cooling process. The trough comprises four blue-detuned Gaussian sheets. Two sheets travel along $\hat{x}$ and form a "V"-shape. Two vertical sheets travel along $\hat{y}$ and serve as end caps sealing the trough along $\hat{x}$. With gravity along $\hat{z}$ this forms a potential capable of trapping atoms.

All four of the sheets used to form the optical trough are derived from the output of the Verdi V10. The optics used to distribute and shape the beam are shown in Fig. 3.21. The Verdi V10 output beam passes through a series of $\lambda / 2$ waveplates and polarizing beam splitters allowing us to split the beam along three paths with the needed intensity ratios. The three paths are 
labeled 1, 2 and 3 in the Fig. 3.21. Beam paths 1 and 2 are used to form the "V"-shaped portion of the optical trough. Each of these beams passes through a telescope formed with a $60 \mathrm{~mm}$ and $200 \mathrm{~mm}$ lens. The beams are spatially filtered at the focus of the $60 \mathrm{~mm}$ lenses. The nominal spacing of the telescope was $260 \mathrm{~mm}$ but the $200 \mathrm{~mm}$ lenses were placed on translation stages, giving control of the beam curvature after the telescope. We used this degree of freedom to adjust the location of the focus of each beam in the optical trough. After passing though the telescope, each beam passes through a cylindrical lens. These two cylindrical lenses were oriented at a right angle to each other so that when the two beams were combined with a polarizing beam splitter they would form the correct "V" shape. After being combined, the two beams were focused into the chamber with a $63.5 \mathrm{~mm}$ lens along the $\hat{x}$ direction. The astigmatism introduced into each beam by the cylindrical lens caused the beams to have $1 / e^{2}$ waists of $10 \mu \mathrm{m} \times 100 \mu \mathrm{m}$ at the location of the optical trough.

The optical end caps were derived from beam path 3 . To produce two end caps we pass this beam through an AOM driven at two frequencies which were nominally $84 \mathrm{MHz}$ and $123 \mathrm{MHz}$. After passing though the AOM unneeded orders were blocked. The unblocked orders passed through a cylindrical lens and were focused into the lower chamber by a $50 \mathrm{~mm}$ lens. The end caps travel along the $\hat{y}$ direction and intersect the two beams which form the "V"-shape. The spacing of the end caps was typically $110 \mu \mathrm{m}$, but by changing the frequencies driving the AOM this could be adjusted. The measured and 
calculated trap depth of this configuration is $\sim 10 \mu \mathrm{K}$. 


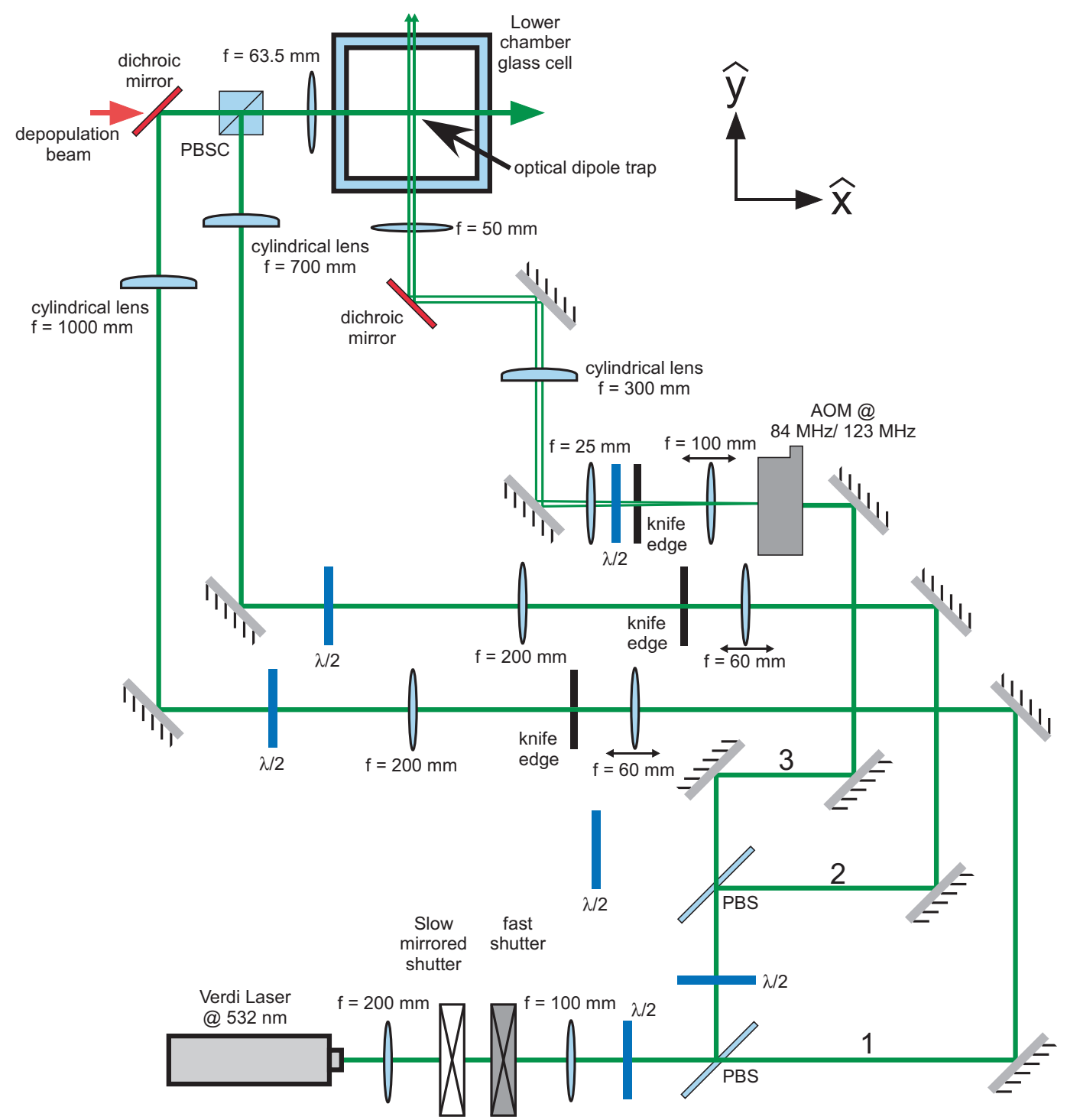

Figure 3.21: Distribution of the Verdi beams. 


\subsection{Magnetic Trap}

Two coils are positioned on either side of the lower vacuum chamber to provide the magnetic field needed to operate the lower MOT and to magnetically trap ${ }^{87} \mathrm{Rb}$ atoms as shown in Fig. 3.22. As discussed earlier, atoms trapped in the upper MOT are pushed through the differential pumping tube and recaptured in the lower MOT. After recapture they are optically pumped into the $\left|F=2, m_{F}=2\right\rangle$ state and trapped magnetically. Magneti-

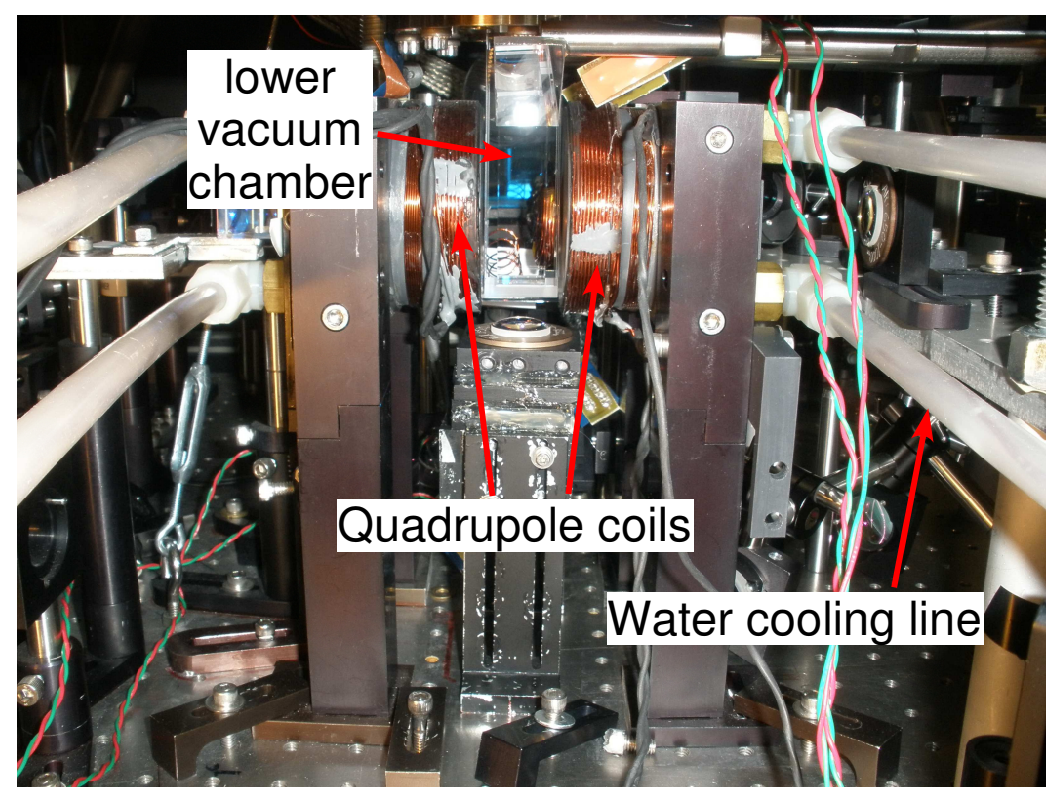

Figure 3.22: Picture of the magnetic trap. Two coils are positioned on either side of the lower vacuum chamber. The wires visible in this image comprise the auxiliary coils and optical pumping coils, while the quadrupole coils are buried beneath. Also visible are the tubes used to pass chilled water over the coils to regulated their temperature.

cally trapped atoms serve as the initial condition for the single-photon cooling 
process.

The two coils are arranged in the simplest trapping geometry possible: the anti-Helmholtz configuration. In this setup two coils are positioned a distance $d$ from each other and carry current of the same magnitude but in the opposite direction. More details on this coil geometry and resultant field can be found in Sec. 2.4.

Figure 3.23 shows one of the coils during the construction process, providing a convenient view of a wound wire sitting on a PVC holder. Each coil was formed from 14 gauge magnetic wire wound around a PVC core a

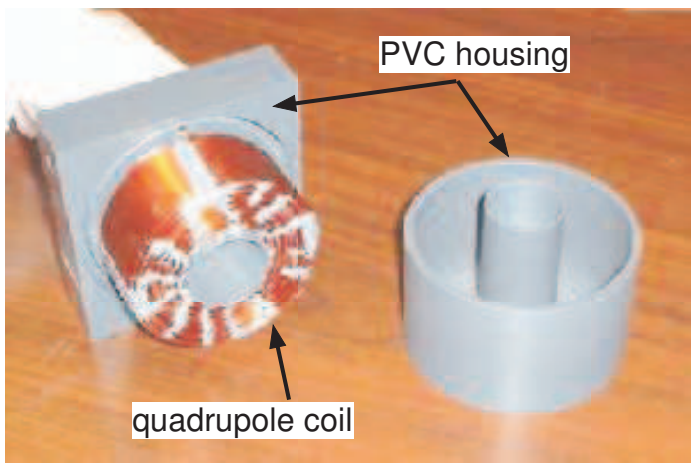

Figure 3.23: A picture of a quadrupole coil during the construction process, providing a convenient view of a wound wire sitting on a PVC holder.

total of 176 times. The wire was wound in three sections, each separated by $1 / 16^{\prime \prime}$ thick nylon spacers. The coil and base were then covered by a PVC lid forming a water tight encasement. This design allows the coils to be cooled by water flowing directly over the wires.

Figure 3.24 is a schematic of the magnetic trap, showing its geometry 
along a horizontal plane through the center of the coils. In this figure the heavily cross-hatched regions indicate wire while the lightly cross-hatched ar-

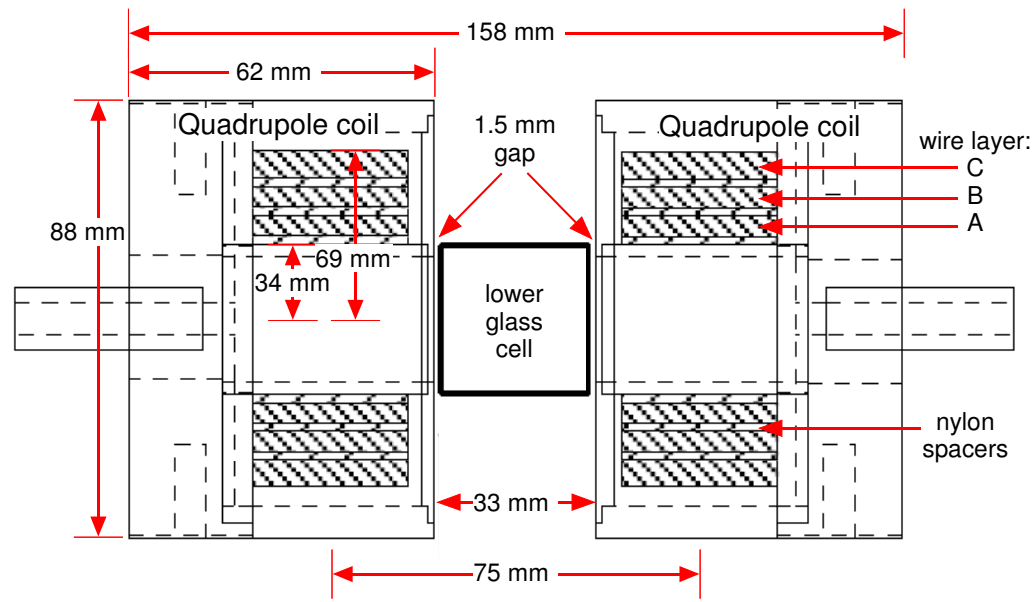

Figure 3.24: Schematic of the magnetic trap showing its geometry along a horizontal plane through the center of the coils.

eas indicate nylon spacers. Wire sections (A) and (B) each consist of a total of 53 turns while section (C) consists of a total of 70 turns. More detail on the construction of these coils can be found in [83, 84].

As discussed in Sec. 2.4, the field near the center of this arrangement is well approximated with a linear gradient whose value varies with direction. The calculated value of the gradient along the axis of symmetry is $9.7 \mathrm{G} /(\mathrm{cm} \mathrm{A})$ and $4.8 \mathrm{G} /(\mathrm{cm} \mathrm{A})$ along the radial direction. These values were calculated using Eq. 2.39 and the geometry in Fig. 3.24 and agree well with measured values [84].

The two coils are in series with three power supplies (Lambda GEN80- 
19) connected in parallel. This arrangement allows us to pass $57 \mathrm{~A}$ of current through the coils, although in practice we never exceeded $30 \mathrm{~A}$. The current was regulated with 7 power op-amps (OPA549) placed in parallel and controlled by a homebuilt PID circuit. The details of this current regulator are found in [84].

Each coil has a resistance of $0.29 \Omega$. With a maximum current of $30 \mathrm{~A}$ running through each, $261 \mathrm{~W}$ of power is dissipated in the form of heat. To remove this heat water continually flowed across the coils and into a commercial water chiller (Neslab MerlinM100) with a total cooling capacity of 3500W.

\subsection{Imaging Systems}

In Sec. 2.8 I discussed the physics of absorption and fluorescence imaging and how we use the raw data collected to calculate values of interest, such as total atom number. This section deals with the origin of the beams used in the imaging process, their beam paths, and the optics and CCD cameras used to record images.

\subsubsection{Vertical Imaging}

The vertical probe beam is derived from the upper MOT horizontal slave laser as discussed in Sec. 3.2.1.3 and shown in Fig. 3.13. This beam has about $3.5 \mathrm{~mW}$ of total power and is tuned to the $|F=2\rangle \rightarrow\left|F^{\prime}=3\right\rangle$ transition frequency. As shown in Fig. 3.25, this beam passes through a $\lambda / 2$ waveplate allowing it to be combined with the push beam using a PBSC. Before entering 


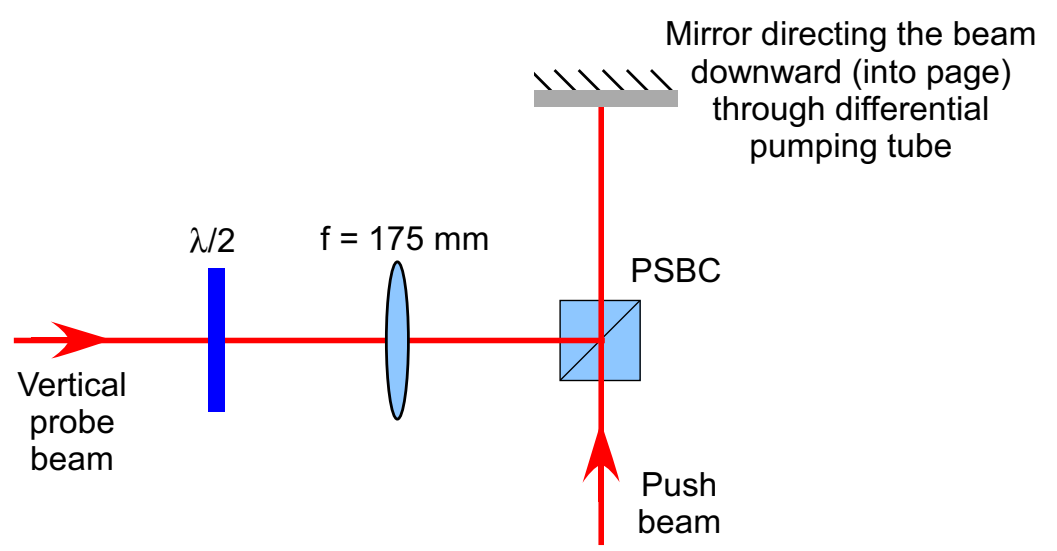

Figure 3.25: Vertical probe beam path. The vertical probe beam passes through a lens and then combines with the push beam with a PBSC. Both beams are directed downwards through the differential pumping tube. After passing through the atomic sample of interest the vertical probe beam is directed towards the imaging optics and CCD camera.

the PBSC, the vertical probe beam passes through a $175 \mathrm{~mm}$ lens which in conjunction with a mirror focuses and directs this beam vertically downward through the differential pumping tube. The beam expands in the differential pumping tube and has a waist of approximately $15 \mathrm{~mm}$ at the location of the atoms. After passing through the atomic cloud the beam is directed to a CCD camera where it is imaged with an objective lens giving a magnification factor of $4.33 \times$. The CCD camera used is an Alta U47+ manufactured by Apogee Instruments, Inc. This camera consists of a $1024 \times 1024$ pixel array (each pixel is $13 \mu \mathrm{m}$ square) cooled with a TEC to $-20^{\circ} \mathrm{C}$ to reduce dark counts. 


\subsubsection{Horizontal Imaging}

The horizontal probe beam is derived from the upper MOT diagonal slave laser as discussed in Sec. 3.2.1.4 and shown in Fig. 3.14. This beam has about $5 \mathrm{~mW}$ of total power, a diameter of $19 \mathrm{~mm}$, and is tuned to the

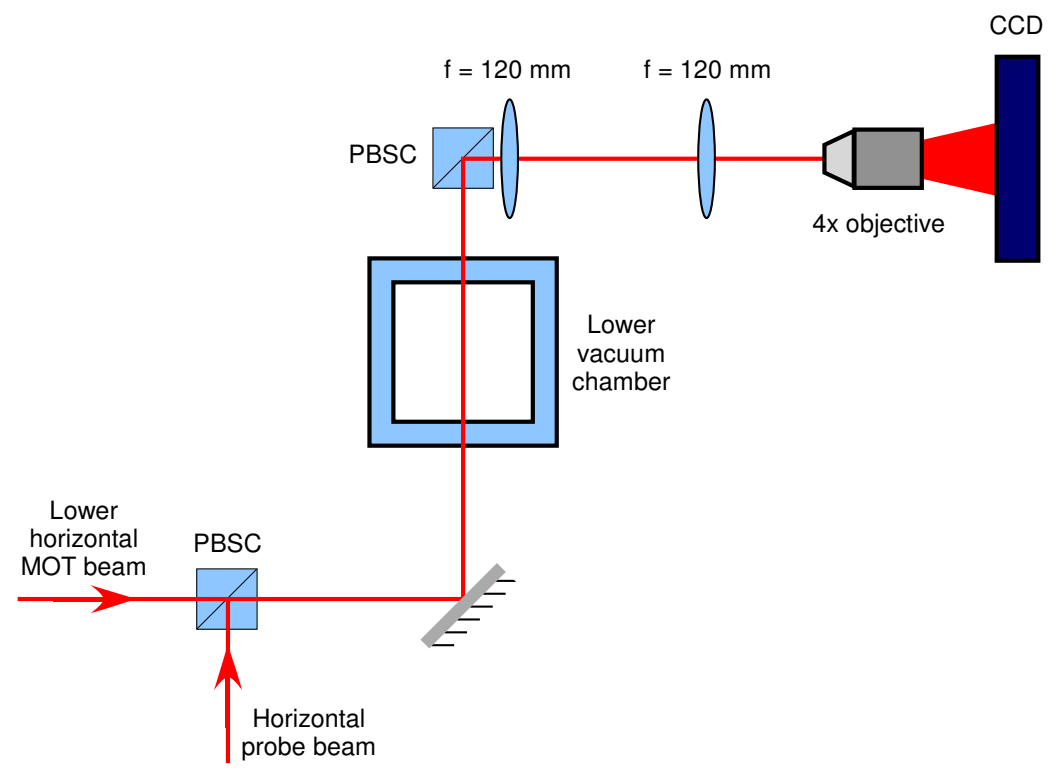

Figure 3.26: Horizontal probe beam path. The horizontal probe beam combines with a horizontal lower MOT beam and passes through the atomic sample. The probe beam is then directed towards the imaging optics and CCD camera.

$|F=2\rangle \rightarrow\left|F^{\prime}=3\right\rangle$ transition frequency. As shown in Fig. 3.26 this beam combines with a horizontal lower MOT beam with a PBSC before entering the lower vacuum chamber. After passing through the cloud, the horizontal probe beam is picked off by a second PBSC and directed to the imaging optics. The beam is relayed by a pair of $120 \mathrm{~mm}$ lenses and imaged on a CCD camera with 
a $4 \times$ objective lens. The camera used for horizontal imaging is an Apogee AP9e. This camera consists of a $3072 \times 2048$ pixel array (each pixel is $9 \mu \mathrm{m}$ square) cooled with a TEC to $-20^{\circ} \mathrm{C}$. 


\section{Chapter 4}

\section{Single-Photon Atomic Cooling in ${ }^{87} \mathrm{Rb}$}

This chapter discusses the application of single-photon atomic cooling to magnetically trapped ${ }^{87} \mathrm{Rb}$. It took several iterations of the experiment to arrive at an apparatus design and procedure which produced cooling limited only by the dynamics of the atoms in the magnetic trap. As such, the specific details of the apparatus and procedure evolved as we learned and improved the process. To make clear to the reader when these changes were made and their effect on the single-photon cooling process, I will present the experimental work in roughly chronological order. To this end, I begin with a discussion outlining the general steps used to cool ${ }^{87} \mathrm{Rb}$ via single-photon atomic cooling common to all experimental iterations. This discussion serves as a starting point for explaining the process and any detail or major deviations from this procedure which we developed will be explained in the text in the appropriate context.

\subsection{Overview of Single-Photon Cooling ${ }^{87} \mathrm{Rb}$}

In this section I move beyond the general descriptions given in Ch. 1 discussing the single-photon cooling process and present a version of the scheme 
used to cool ${ }^{87} \mathrm{Rb}$ experimentally, emphasizing those features common to all iterations of the experiment. This section represents an updated version of our original paper proposing a single-photon cooling scheme appropriate for ${ }^{87} \mathrm{Rb}$ [94]. In all iterations of the experimental process a sample of magnetically trapped ${ }^{87} \mathrm{Rb}$ was transfered into a conservative optical dipole trap via the single-photon cooling process. The shape and construction of the optical dipole trap varied with each iteration. For the sake of simplicity, I will use a generic optical potential in this overview of the single-photon cooling process and describe the details of each iteration in later sections.

To obtain the magnetically trapped sample of ${ }^{87} \mathrm{Rb}$, several steps are taken. Atoms are initially loaded into the upper MOT from room temperature ${ }^{87} \mathrm{Rb}$ vapor. A discussion of the design of the upper chamber and source of $\mathrm{Rb}$ can be found in Sec. 3.1.1. As the upper MOT is loaded, the push beam transfers ${ }^{87} \mathrm{Rb}$ atoms into the lower chamber where they are recaptured in the lower MOT. When optimized, we are able to collect atoms in the lower MOT at a rate of roughly $10^{8}$ atoms/sec. This rate drops off after a few seconds due to saturation effects. When loading the lower MOT we run approximately $1.6 \mathrm{~A}$ of current through the lower magnetic quadrupole coils thereby producing a magnetic field gradient of $B^{\prime}=8 \mathrm{G} / \mathrm{cm}$ (see Sec. 2.4 and Sec. 3.3 for more details). The lower MOT beams are typically detuned $-15 \mathrm{MHz}$ from the $|F=2\rangle \rightarrow\left|F^{\prime}=3\right\rangle$ cycling transition, have a waist of $\sim 1 \mathrm{~cm}$ and an optical power of $7 \mathrm{~mW}$ each.

We typically operated the lower MOT loading stage for approximately 
$1 \mathrm{~s}$, after which time $\sim 10^{8}{ }^{87} \mathrm{Rb}$ atoms were present. To efficiently transfer these atoms into the magnetic trap they underwent two more preparatory steps in the lower chamber. The first of these is optical molasses (see Sec. 2.5.2.2). This step reduces the temperature of the atoms from 100-150 $\mu \mathrm{K}$ to approximately $10 \mu \mathrm{K}$. During the optical molasses stage the MOT beams are reduced in intensity by approximately $1 / 2$, the frequency detuning is increased to $-50 \mathrm{MHz}$ from the $|F=2\rangle \rightarrow\left|F^{\prime}=3\right\rangle$ transition frequency, and the magnetic field is shut off. The duration of this stage was normally $5 \mathrm{~ms}$, although, as will be seen in Sec. 4.4, we adjusted the duration to control the temperature of the magnetically trapped atoms. Optical molasses leaves the internal state of the atoms distributed among the various Zeeman magnetic sublevels. To maximize loading into the magnetic trap we optically pump atoms into the $\left|F=2, m_{F}=2\right\rangle$ state. This is accomplished by producing a weak, uniform magnetic field with the quadrupole auxiliary coils to define a quantization axis. Then a $\sigma^{+}$polarized beam tuned to resonance with the $|F=2\rangle \rightarrow\left|F^{\prime}=2\right\rangle$ transition frequency is sent along this axis, driving atoms into the $\left|F=2, m_{F}=2\right\rangle$ dark state. This process is very fast, taking place in approximately $100 \mu \mathrm{s}$, and results in an increased transfer efficiency at the price of slightly heating $(\sim 5 \mu \mathrm{K})$ the atomic sample.

After being magneto-optically trapped, cooled, and placed into the $\left|F=2, m_{F}=2\right\rangle$ state, the atoms are ready to be magnetically confined. This is accomplished by turning off all optical fields and ramping the current in the magnetic quadrupole coils to $10 \mathrm{~A}$ in a few milliseconds. This cur- 
rent results in a magnetic field gradient of $B^{\prime}=48 \mathrm{G} / \mathrm{cm}$. Mode mismatch between the optically pumped atomic sample and the magnetic trap results in heating. The final temperature of the magnetically trapped sample was typically in range of $50-100 \mu \mathrm{K}$. Under these conditions, the $1 / e^{2}$ radius of the magnetically trapped atomic cloud was $\sim 500 \mu \mathrm{m}$. These atoms serve as the initial condition for further cooling via the single-photon cooling process during which time they are transfered into an optical trap.

Then next step in the single-photon cooling process involves positioning a conservative optical dipole trapped in the wings of the magnetically trap sample. As stated in the introduction to this chapter, the details of the optical dipole trap evolved as the experiment progressed. To keep this overview simple, I will introduce an optical trap representative of the actual potentials used which encompasses their key features. The details of each iteration of the optical trap used during the experiments are discussed in the next several sections. For now, consider a blue detuned optical sheet placed below the magnetically trapped atomic sample. Because the sheet is tuned below the atomic resonance frequency, it produces a repulsive barrier. This barrier is capable of levitating the atoms with sufficiently high optical intensity. Figure 4.1(a) illustrates the geometry under consideration. Figure 4.1(b) shows the potential landscape for atoms in the $\left|F=2, m_{F}=2\right\rangle$ state due to the magnetic field gradient, gravity, and the optical sheet. For comparision, Fig. 4.1(c) shows the potential landscape for atoms in the $\left|F=1, m_{F}=0\right\rangle$ state due to gravity and the optical sheet. Notice that atoms in this state are decoupled, to first 
(a)
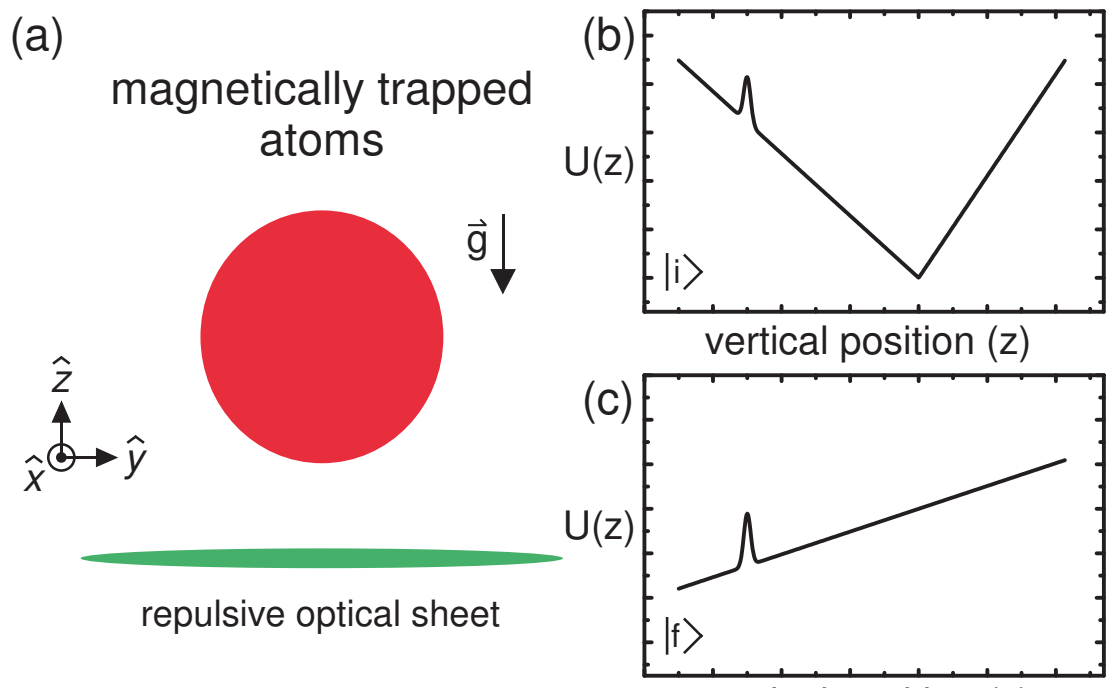

vertical position ( $\mathrm{z})$

Figure 4.1: Overview of the single-photon cooling process. (a) Cross-sectional view of magnetically trapped atoms above a repulsive optical sheet. (b) Effective potential along the vertical $(\hat{z})$ axis for atoms in the $\left|F=2, m_{F}=2\right\rangle$ state due to the combined potentials of a quadrupole magnetic field, gravity, and a repulsive optical sheet. (c) Potential for atoms in the magnetically decoupled $\left|F=1, m_{F}=0\right\rangle$ state.

order, from the magnetic field and the tilt in the potential is due entirely to gravity.

The idea behind single-photon atomic cooling is to transfer the magnetically trapped atoms, which are in the $\left|F=2, m_{F}=2\right\rangle$ state, into the magnetically decoupled $\left|F=1, m_{F}=0\right\rangle$ state preferentially when they are near their classical turning point and close to the optical sheet. There are two key ideas here that I want to make clear. First, by transferring the atoms from the magnetically coupled to the magnetically decoupled state near their 
turning points, they will have little residual kinetic energy. After the state transfer the atoms will fall onto the optical sheet which holds them against gravity. Second, by transferring the atoms near the sheet they will acquire little energy due to free fall. The state transfer is accomplished optically with the depopulation beam (see Sec. 3.2.1.7).

The depopulation beam is tuned near the $|F=2\rangle \rightarrow\left|F^{\prime}=1\right\rangle$ transition frequency. Correspondingly, its function is to transfer atoms from the $\mid F=$ $\left.2, m_{F}=2\right\rangle$ state into the excited $\left|F^{\prime}=1, m_{F^{\prime}}=1\right\rangle$ state. Once excited, the atoms quickly decay ( $\tau \sim 26 \mathrm{~ns}$ ). Calculation of the decay branching ratio is discussed in Sec. 2.6 and the results are summarized in Fig. 2.12. As indicated in this figure we can optically pump $42 \%$ of the atoms into the $\left|F=1, m_{F}=0\right\rangle$ state in a single cycle. Experimentally we do somewhat better because any atom which decays into the $F=2$ manifold is reexcited by the depopulation beam and has another chance to decay into the $F=1$ manifold. We find close to $1 / 2$ of the atoms decay into the desired $\left|F=1, m_{F}=0\right\rangle$ state.

Ensuring that the atoms are transfered into the magnetically decoupled state near the repulsive optical sheet is a simple matter. One only needs to form a second optical sheet from the depopulation beam and place it slightly above the repulsive sheet (see Fig.4.2). Transferring the atoms preferentially at their turning points is also accomplished in a straightforward manner. In this geometry, one would initially place the two sheets below the magnetic trap beyond the turning point of even the most energetic atom and then slowly sweep the pair of sheets vertically towards the center of the magnetic trap. If 


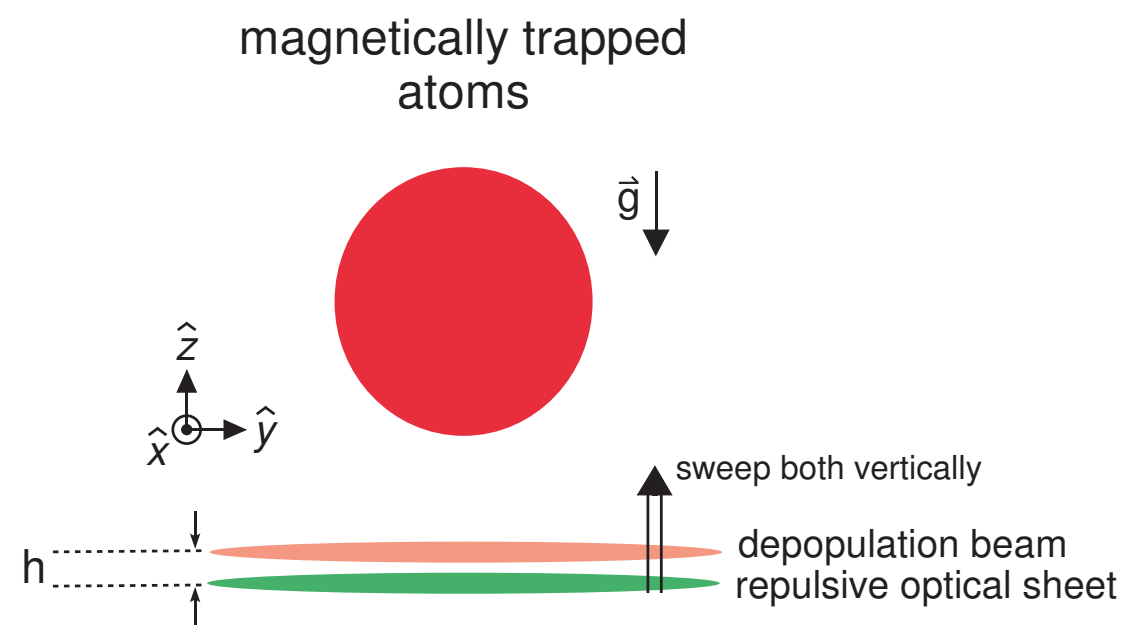

Figure 4.2: Depopulation sheet positioned a distance $h$ above the repulsive sheet. To cool, both sheets are slowly swept vertically towards the center of the magnetic trap center.

done slowly (see Sec. 1.4 for a discussion on the relevant time scales) the atoms first encounter the depopulation beam near their turning points. The reader may be alarmed because in this description the optical sheet only confines the atoms vertically. There is no cause for alarm, because as described in Secs. 4.2-4.4, the actual optical potentials used during the experiment formed 3-D confining potentials.

The following three sections outline the development and experimental results of this cooling process. Each experimental iteration shares its main features with the description just given, only differing in details which will be explained in context. 


\subsection{Crossed Dipole Beam Configuration}

This section reports on our first experimental implementation of singlephoton atomic cooling with a magnetically trapped sample of ${ }^{87} \mathrm{Rb}$. While the results presented in this section did not merit publication, they did offer the first evidence that the single-photon cooling process worked. We felt encouraged that with improvements to the experimental apparatus and process, single-photon atomic cooling could be made an efficient method. Because of this, these results are presented with somewhat less rigor than in the next two sections which represent published work. In this experiment, as in the later iterations, we transfered atoms initially in the $\left|F=2, m_{F}=2\right\rangle$ state from a large volume magnetic trap into a smaller volume optical dipole trap via the single-photon cooling process.

During this iteration of the experiment the optical dipole trap was formed from two crossed laser beams at a wavelength of $1064 \mathrm{~nm}$. Each beam originated from a multi-mode ytterbium fiber laser with a maximum output power of $10 \mathrm{~W}$ and a spectral bandwidth $<1 \mathrm{~nm}$. This optical trap was placed above magnetically trapped atoms as shown in Fig. 4.3. Because the laser output frequency is well to the red of the $\mathrm{D}$ transition lines in $\mathrm{Rb}$, it formed an attractive potential whose depth can be found from Eq. 2.53 and knowledge of the trap geometry. Figure 4.4 shows the calculated potential landscape for

atoms in the $\left|F=2, m_{F}=2\right\rangle$ state due to the magnetic and optical traps as well as gravity. In this figure, parameters consistent with experimental values are assumed. The current in the magnetic coils is $15 \mathrm{~A}$, resulting in 


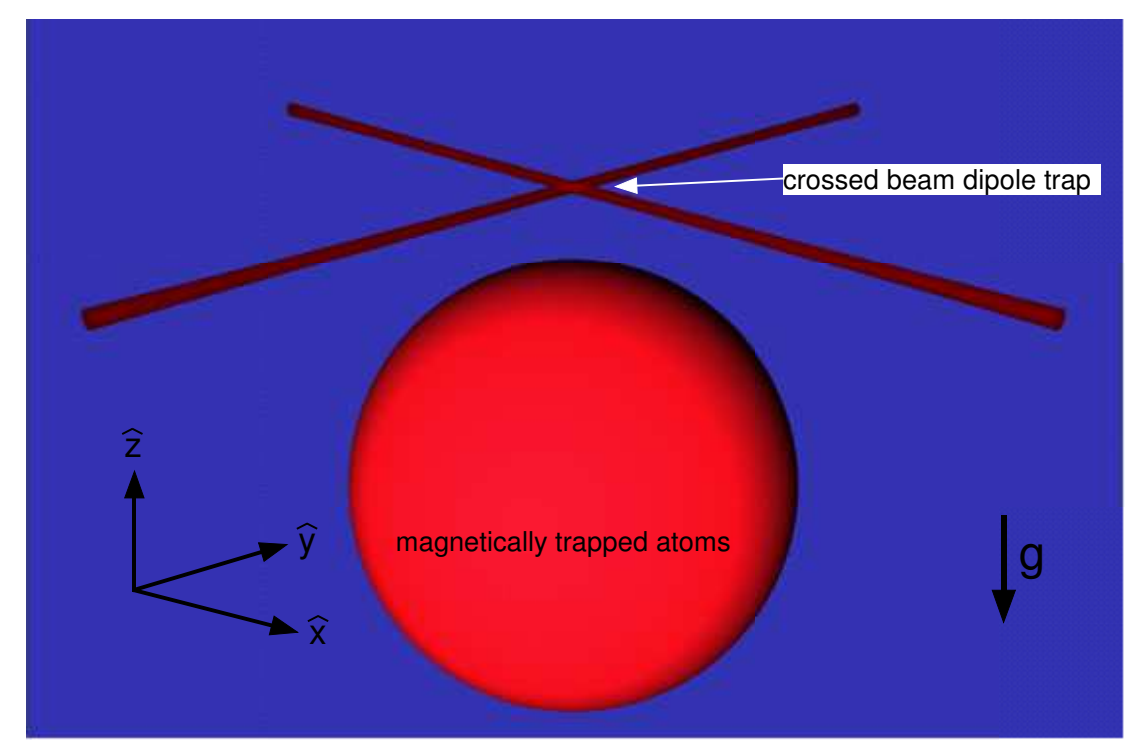

Figure 4.3: Schematic of crossed laser beams above a cloud of magnetically trapped atoms. Gravity is in the $-\hat{z}$ direction as shown in the figure.

a magnetic field gradient of $B^{\prime}=72 \mathrm{G} / \mathrm{cm}$ (see Sec. 2.4 for a definition of this constant). Each dipole beam had $3.5 \mathrm{~W}$ of power and a $1 / e^{2}$ waist of approximatly $150 \mu \mathrm{m}$.

As shown in Fig. 4.4 a small dimple is evident on the right hand side of the potential, however it is too small to create a bound state. Observe that another state, $\left|F=1, m_{F}=1\right\rangle$, does have a bound state under identical conditions. The potential landscapes for the two states are shown in Fig. 4.5. The reason for formation of the bound state is that the magnetic tilt is only half of the value of the $\left|F=2, m_{F}=2\right\rangle$ state.

To transfer atoms from the magnetic trap into the optical trap, we added a depopulation beam parallel to a dipole beam. During the experimen- 


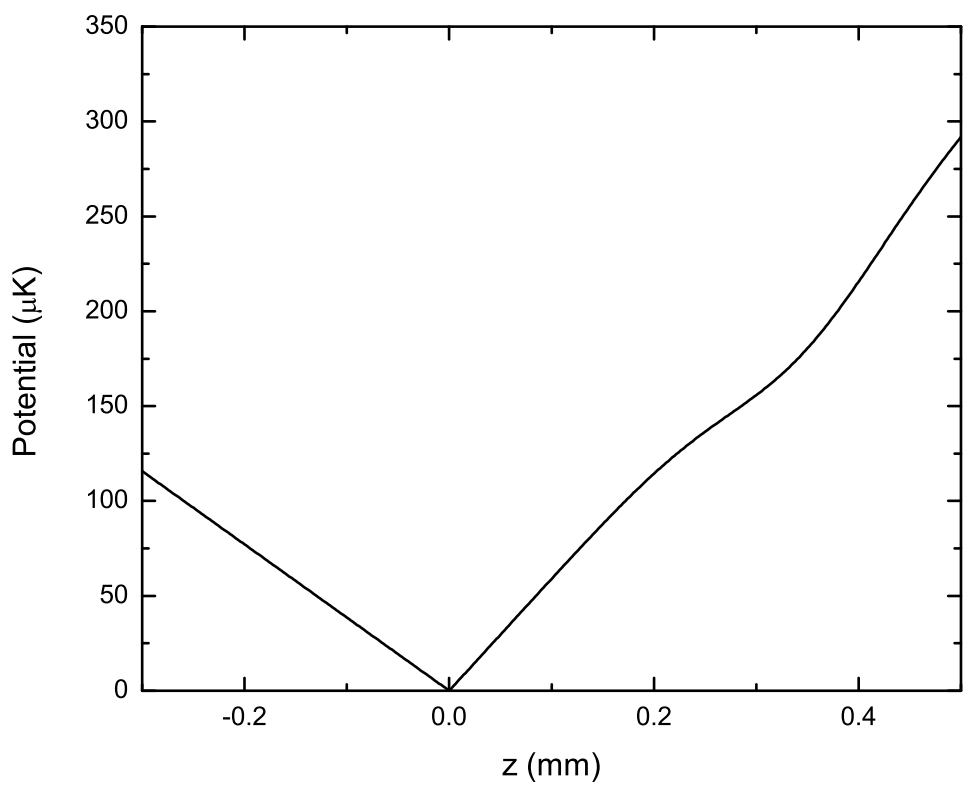

Figure 4.4: The potential landscape for atoms in the $\left|F=2, m_{F}=2\right\rangle$ state due to the magnetic and optical traps as well as gravity.

tal runs reported here, the depopulation beam had approximately $75 \mathrm{nW}$ of power, a vertical $1 / e^{2}$ waist of $16 \mu \mathrm{m}$, and a horizontal waist of $54 \mu \mathrm{m}$. This corresponds to a peak intensity of $5.5 \mathrm{~mW} / \mathrm{cm}^{2}$, about twice the transition saturation intensity. As indicated in Fig. 4.6 the position of the depopuation beam relative to the optical dipole beams is an important parameter. If not positioned correctly, transfered atoms will gain energy as they fall down the potential hill, possibly leading to trap loss. We found that offsetting the depopulation beam $\sim 30 \mu \mathrm{m}$ below the center of the optical dipole beams yielded the largest transfer of atoms.

At the start of the cooling process the centers of the magnetic trap and 


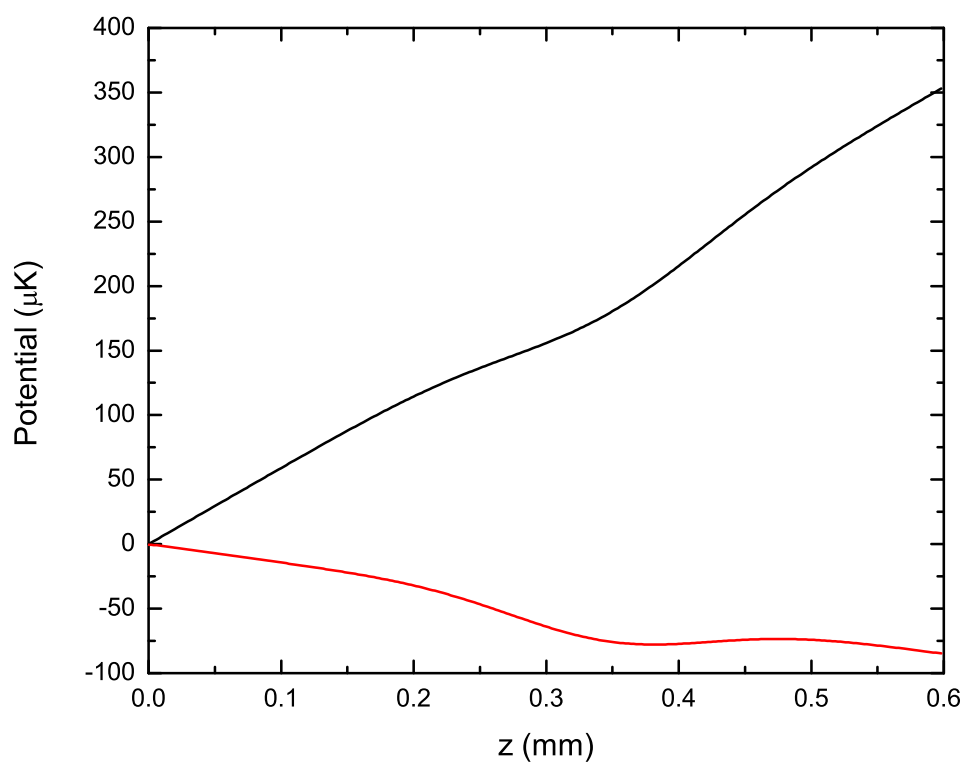

Figure 4.5: The effective potential for the combined optical and magnetic traps as well as gravity for atoms in the $\left|F=2, m_{F}=2\right\rangle$ state (upper black line) and atoms in the $\left|F=1, m_{F}=1\right\rangle$ state (lower red line).

optical dipole trap were separated by $\sim 750 \mu \mathrm{m}$. To initiate the single-photon cooling process the magnetic trap was moved upwards, towards the center of the dipole trap, by linearly ramping the current in a coil positioned above the magnetically trapped atoms. Through experimentation we found that a ramp duration of 0.5 - $1 \mathrm{~s}$ transfered the most atoms into the optical dipole trap via the single-photon cooling process. As will be discussed in more detail later, this ramp rate struck the optimal balance between allowing the atoms to explore phase space and limiting trap loss due to heating and other effects.

To image and count the transfered atoms, we isolated them from those which had not undergone the single-photon cooling process. First, all mag- 


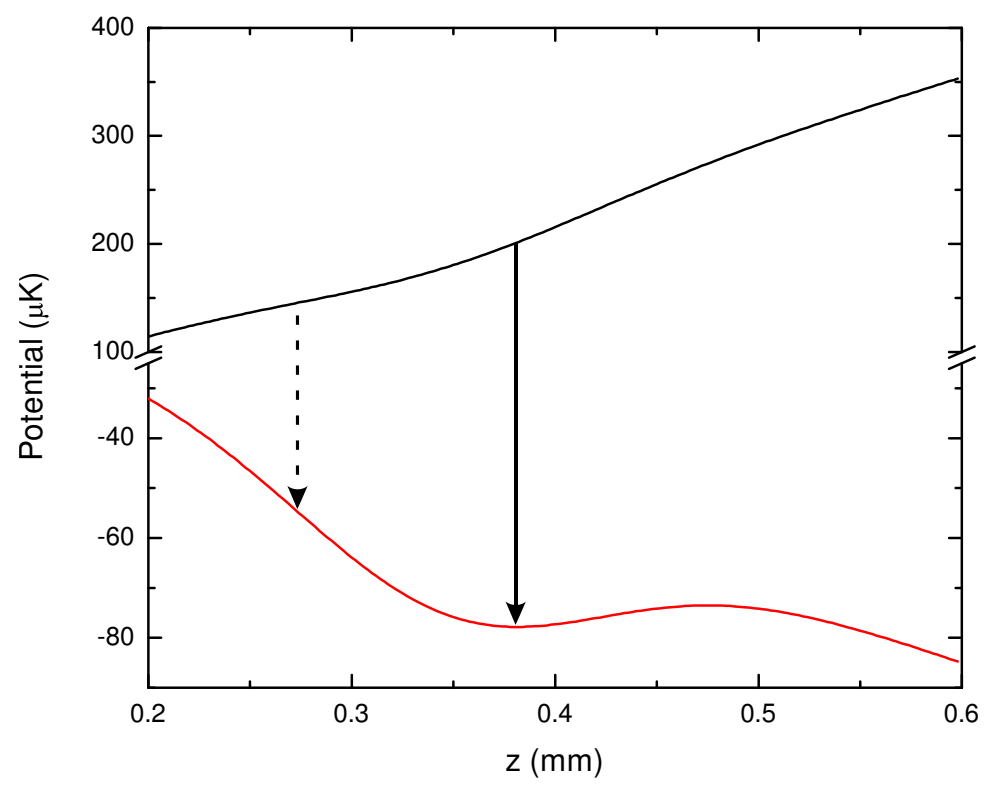

Figure 4.6: Transition induced by the depopulation beam, depending on the spatial location of the beam. The solid line is the preferred location and will lead to less heating than the dashed line.

netic fields were shut off to allow those atoms not optically trapped to fall for $80 \mathrm{~ms}$ under the influence of gravity. Then we blew away any residual atoms in the $F=2$ manifold by application of a beam resonant with the $|F=2\rangle \rightarrow|F=3\rangle$ transition. The remaining atoms had undergone the single-photon cooling process. These atoms were repumped into the $F=2$ manifold and placed in freezing molasses. The resultant fluorescence light was collected and imaged on a CCD camera. Figure 4.7 indicates the results of this experiment. Figure 4.7(a) was taken with no depopulation beam present during the experimental sequence. As expected, this image indicates that no atoms were transfered into the optical trap. Figure 4.7(b) was taken with the 
depopulation present and it indicates that atoms were transfered during the

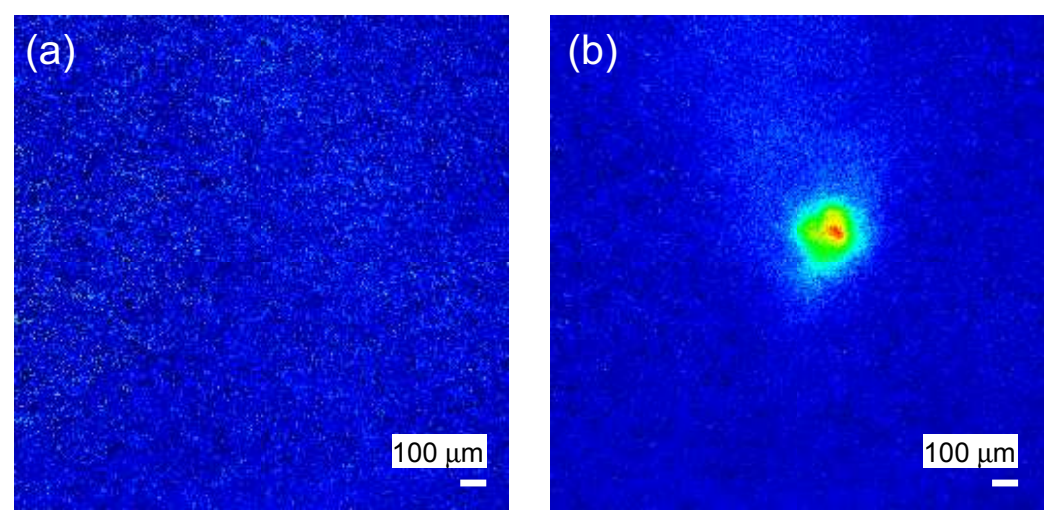

Figure 4.7: Fluorescence image of atoms transfered into the optical dipole trap via the single-photon cooling process (a) without and (b) with the depopulation beam present. In these images false color is used to represent atomic density. Red represents the densest region.

single-photon cooling process. This image indicates that the optically trapped atomic cloud had a $1 / e$ radius of roughly $123 \mu \mathrm{m}$, consistent with the known dipole beam waists of $150 \mu \mathrm{m}$. Additionally, time-of-flight measurements (see Sec. 2.8) indicate the temperature of the transfered sample to be $12 \mu \mathrm{K}$. Figure 4.8 shows the raw data used to measure this temperature. Unfortunately, the absolute atom number could not be determined because the number of transfered atoms was too small for our system to measure.

This work served as experimental evidence that the single-photon cooling process worked and gave us encouragement that with improvements we could expect to transfer a larger number of atoms. Indeed, the next two sections discuss experimental iterations with greatly improved performance, 


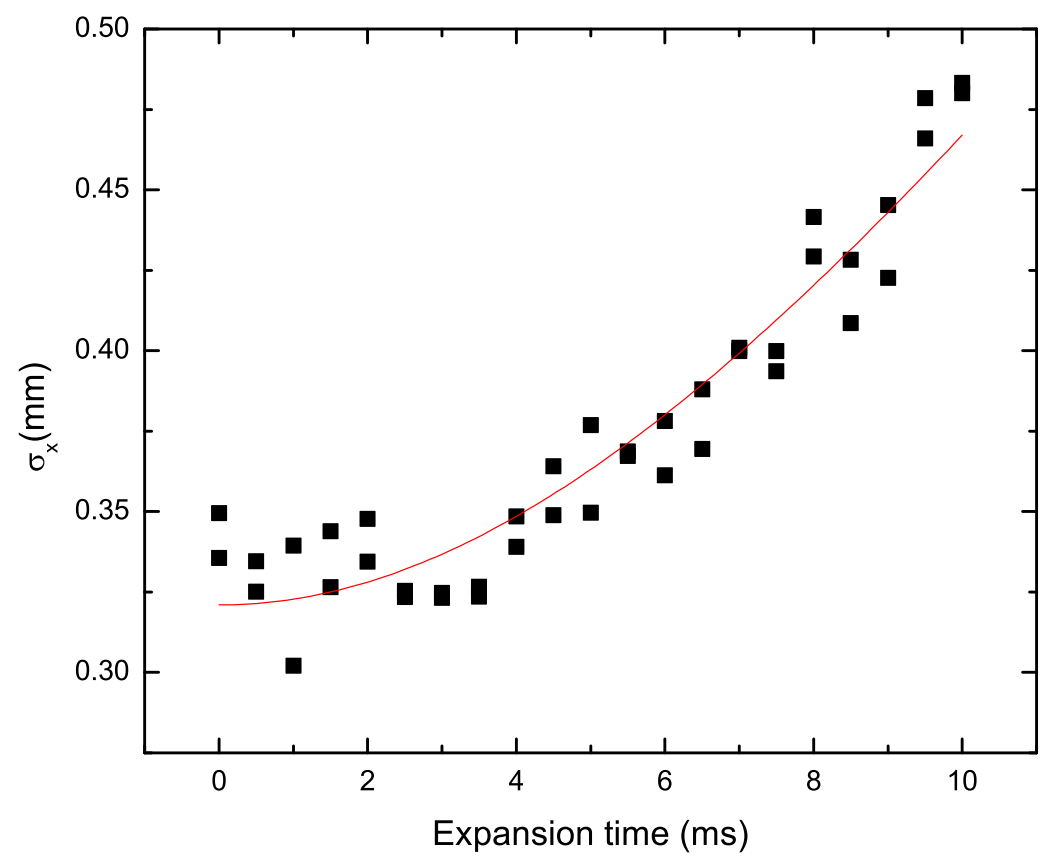

Figure 4.8: Width of atomic distribution as a function of expansion time for atoms cooled via the single-photon cooling process in the cross beam configuration. Black squares indicate raw data while the red line shows the best fit. See Sec. 2.8.1 for details on the function used to fit this data.

allowing for far more quantitative analysis.

The primary limitation in this iteration of the experiment was the short lifetime of the optical dipole trap $(\sim 0.7 \mathrm{~s})$. This lifetime set the timescale over which we could accumulate and hold atoms. After an unsuccessful attempt at increasing the lifetime of this attractive optical dipole trap we decided to use our Verdi V10 laser (see Sec. 3.2.2) to form a repulsive trap to transfer the atoms into. Use of this technique did result in a substantial increase in the lifetime of optically trapped atoms $(\sim 3.7 \mathrm{~s})$, allowing us to accumulate atom 
numbers sufficiently large to quantify. This work serves as the topic of the next section.

\section{3 "Optical Box" Configuration}

This section discusses the second major experimental iteration [95]. The major improvement made in this iteration was a change in the construction of the optical dipole trap. We stopped using the multi-mode ytterbium fiber lasers $(1064 \mathrm{~nm})$ to form an attractive potential and began using the Verdi V10 $(523 \mathrm{~nm})$ to form a repulsive potential to transfer the atoms into. The initial steps of loading atoms in to the magnetic trap was the same as that used in the previous section and described in Sec. 4.1.

A thermal cloud of ${ }^{87} \mathrm{Rb}$ atoms is initially produced in a magnetooptical trap and then cooled in optical molasses. Subsequently atoms in the $|F=2\rangle$ hyperfine ground state are loaded into a magnetic quadrupole trap with a radial field gradient of $75 \mathrm{G} / \mathrm{cm}$. We trap approximately $1.7 \times 10^{8}$ atoms at a temperature of $90 \mu \mathrm{K}$ in a cloud with a $1 / e$ radius of $550 \mu \mathrm{m}$.

After the magnetic trap is loaded, an optical dipole trap is positioned above it. The optical dipole trap originates the Verdi V10 which is split into three beams. Each beam passes through a dual-frequency acousto-optic modulator, and the first order deflections are tightly focused in one dimension

to form parallel sheets. Each individual sheet has a $1 / e^{2}$ beam waist of $10 \mu \mathrm{m}$ $\times 200 \mu \mathrm{m}$ and a power of $0.7 \mathrm{~W}$. The three pairs of sheets are crossed to form a repulsive "box-like" potential, with dimensions $100 \mu \mathrm{m} \times 100 \mu \mathrm{m} \times 130 \mu \mathrm{m}$ 
and a depth of $k_{B} \times 10 \mu \mathrm{K}$, shown pictorially in Fig.4.9(a).

\section{(a)}

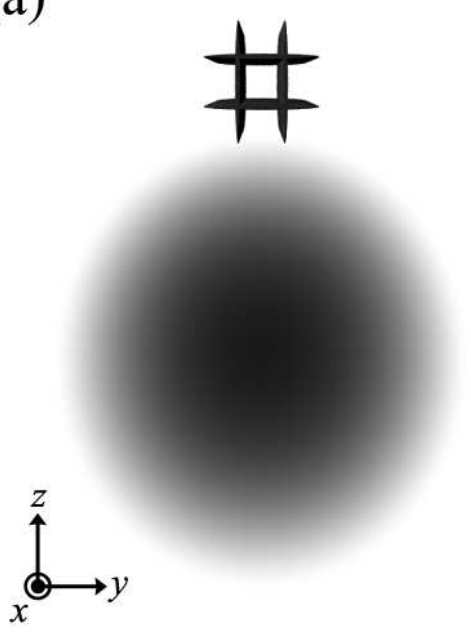

(b)

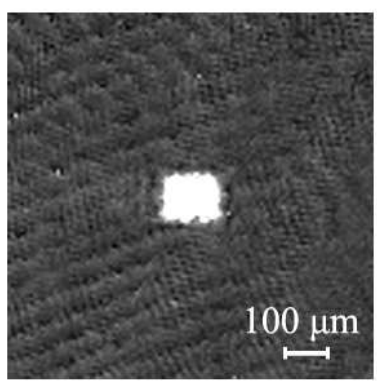

Figure 4.9: (a) Cross-section of the optical box positioned above the cloud of magnetically trapped atoms. In this illustration, two pairs of Gaussian laser sheets propagate parallel to the x-axis. A third pair (not visible) propagates parallel to the y-axis and completes the optical box. (b) Absorption image along the z-axis of approximately $1.5 \times 10^{5}$ atoms trapped in the optical box.

In this cross sectional view only two of the three pairs of sheets are visible. The pair confining the atoms along the $\hat{z}$ (vertical) direction are referred to as the horizontal sheets. Likewise, the pair confining the atoms along the $\hat{y}$ direction are referred to as the vertical sheets. Both the horizontal and vertical sheets propagate along the $\hat{x}$ axis. To confine the atoms along the $\hat{x}$ direction, two more sheets elongated vertically propagate along the $\hat{y}$ axis serving as "end caps."

Figure 4.10 shows the calculated optical dipole potential due to the 

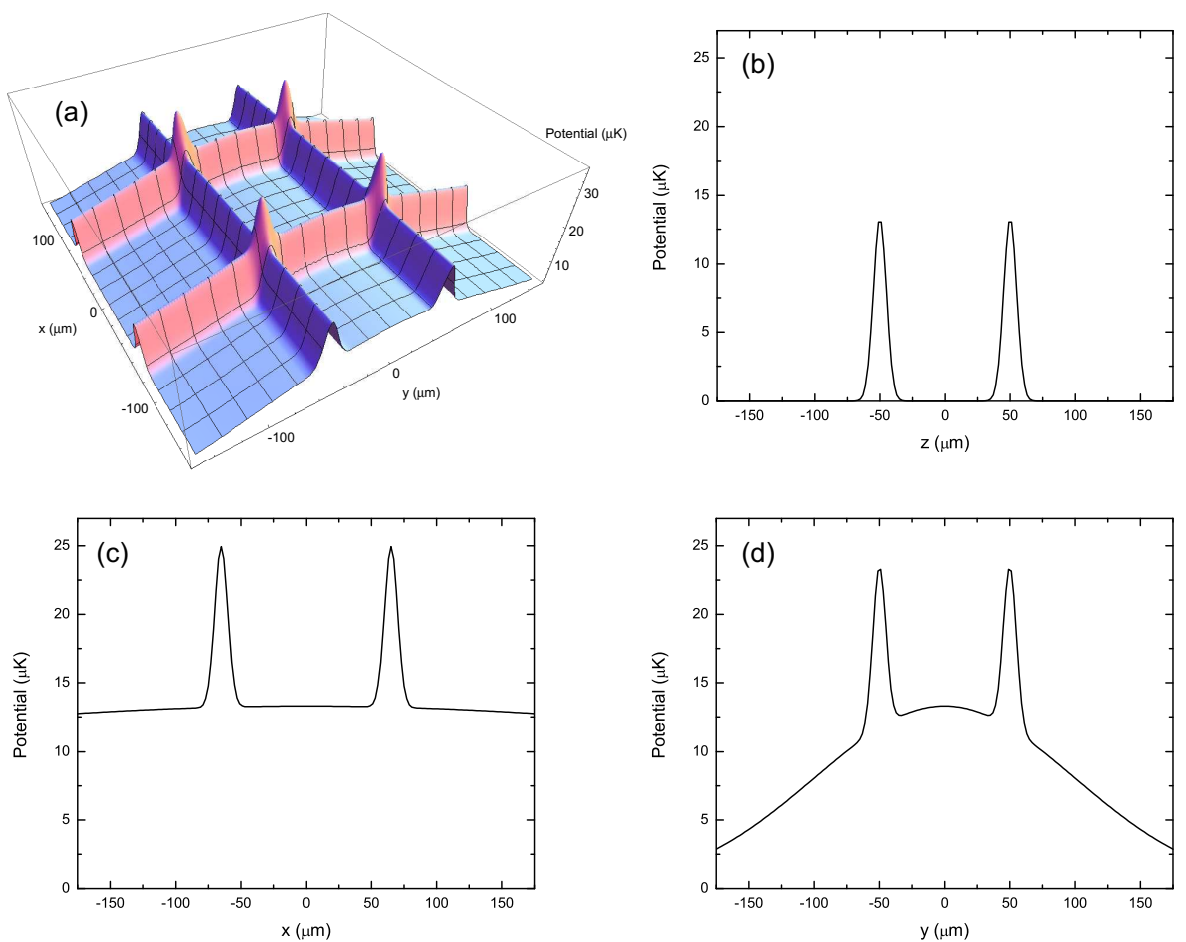

Figure 4.10: Calculated optical dipole potential due to the optical box under experimental conditions. (a) 2-D slice of the optical dipole potential in the $\mathrm{x}-\mathrm{y}$ plane located at $z=-50 \mu \mathrm{m}$. (b) Potential along $\hat{z}$ with $x=y=0$. (c) Potential along $\hat{x}$ with $y=0$ and $z=-50 \mu \mathrm{m}$. (d) Potential along $\hat{y}$ with $x=0$ and $z=-50 \mu \mathrm{m}$.

optical box under experimental conditions. Quadrant (a) of Fig. 4.10 displays a 2-D slice of the calculated optical dipole potential in an $\mathrm{x}-\mathrm{y}$ plane located at $z=-50 \mu m$ (the location of the center of the lower horizontal sheet). The central well present in this figure is used to confine the transfered atoms. Figure 4.10(b) displays the calculated optical dipole potential along the $\hat{z}$ direction midway between the vertical sheets and end caps $(x=y=0)$. The two peaks in this graph reflect the potential due to upper and lower horizontal 
sheets. Figure 4.10(c) shows the calculated potential along the $\hat{x}$ direction with $y=0$ and $z=-50 \mu \mathrm{m}$. Similarly, the two peaks reflect the potential due to the end caps. The slight curvature of the potential (away from the peaks) is due to the finite Rayleigh length of the lower horizontal sheet. This sheet is focused asymmetrically and therefore has a Rayleigh length associated with each transverse dimension. The Rayleigh length associated with the tight vertical focusing is $Z_{R}=590 \mu \mathrm{m}$. Finally, Fig. 4.10(d) shows the potential along the $\hat{y}$ direction with $x=0$ and $z=-50 \mu \mathrm{m}$. Here, the two peaks are due to the vertical sheets. The pronounced curvature of the potential (away from the peaks) is due to the $200 \mu \mathrm{m}$ horizontal beam waist of the lower horizontal sheet.

The accumulation of atoms into the optical box, a conservative trap, requires an irreversible step. This need is met by optically pumping the atoms that transit the optical box to the $F=1$ manifold with the depopulation beam. The beam is near resonant with the $5 S_{1 / 2}(F=2) \rightarrow 5 P_{3 / 2}\left(F^{\prime}=1\right)$ transition and focused to a $1 / e^{2}$ waist of $8 \mu \mathrm{m} \times 200 \mu \mathrm{m}$ at the center of the box. Magnetically trapped atoms in the $F=2$ manifold are excited by the depopulation beam and decay with $84 \%$ probability to the $F=1$ manifold $\left(m_{F}=1,0\right)$, where they are no longer on resonance with the depopulation beam. Because the gradient of the Zeeman shift of these states is smaller than that of the initial state, the contribution from the magnetic field to the total potential is reduced, creating a trapped state in the optical box in manner identical to that discussed in the previous section. Figure 4.11 show 
the potential landscape due to the combined magnetic field, optical dipole trap, and gravity for atoms in the $\left|F=2, m_{F}=2\right\rangle$ and $\left|F=1, m_{F}=0\right\rangle$ states, as well as the desired position of the state transfer.

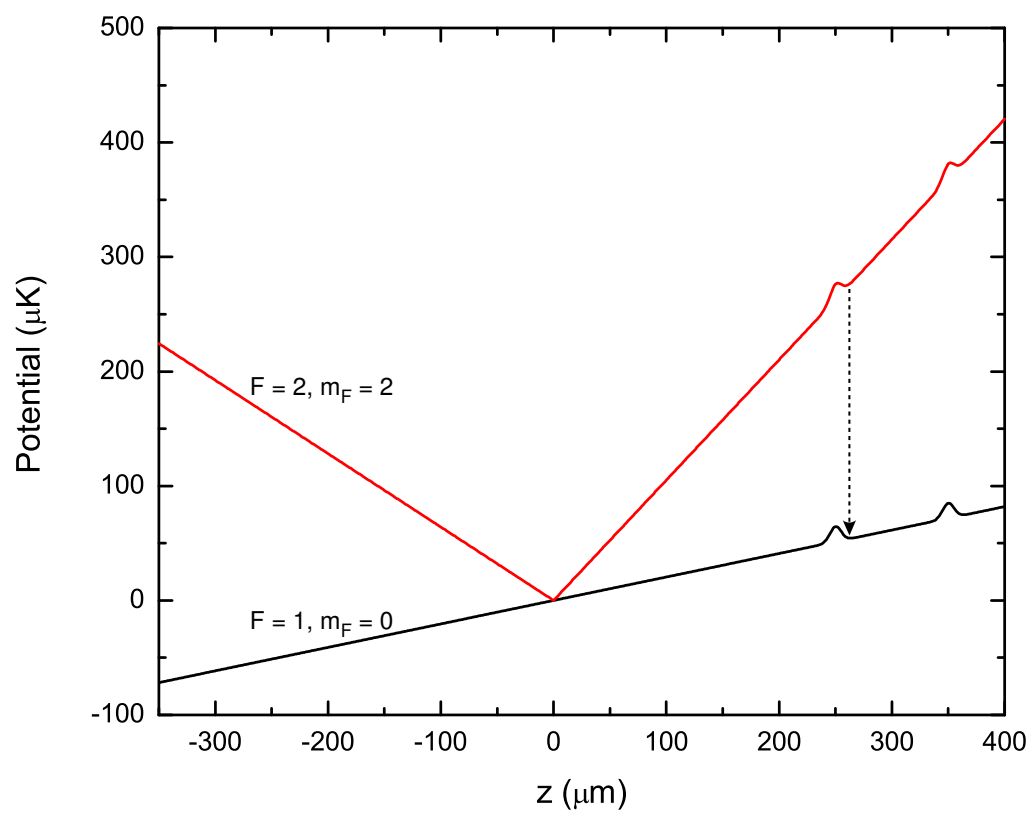

Figure 4.11: The potential landscape due to the combined magnetic field, optical dipole trap, and gravity for atoms in the $\left|F=2, m_{F}=2\right\rangle$ and $\mid F=$ $\left.1, m_{F}=0\right\rangle$ states. Atoms are transfered from the $\left|F=2, m_{F}=2\right\rangle$ state into the $\left|F=1, m_{F}=0\right\rangle$ state by the depopulation beam at the position indicted by the dashed vertical line.

As atoms accumulate in the optical box, the outermost trajectories of the magnetic trap are depleted by the depopulation beam. For maximum loading into the optical box, we adiabatically translate the center of the magnetic trap towards the optical box by applying a linear current ramp to an auxiliary magnetic coil located above the atoms. 
Before imaging, we isolate the optically trapped atoms by switching off the magnetic trap, allowing untrapped atoms to fall under the influence of gravity for $80 \mathrm{~ms}$. Additionally, the depopulation beam is turned off and a beam resonant with the $5 S_{1 / 2}(F=2) \rightarrow 5 P_{3 / 2}\left(F^{\prime}=3\right)$ transition blows away any residual atoms in the $F=2$ manifold. The remaining atoms are those which have undergone single-photon atomic cooling. These atoms are pumped to the $F=2$ manifold and illuminated with freezing molasses for $30 \mathrm{~ms}$. The resulting fluorescence is imaged on a charge-coupled device (CCD) camera and integrated to yield atom number. Spatial information is obtained by imaging with absorption rather than fluorescence as in Fig. 4.9(b).

The density of atoms loaded into the optical box via single-photon atomic cooling is sensitive to multiple parameters. The intensity of the depopulation beam strongly affects the final density; it must be set to balance efficient pumping into the $F=1$ manifold with trap loss due to heating and other loss mechanisms. A more detailed discussion of this point is given in the next section. Figure 4.12 shows a plot of the number of atoms loaded into the optical box via single-photon atomic cooling as a function of the power in the depopulation beam. In this experimental configuration, we maximize density in the optical box with a peak depopulation beam intensity of approximately $8 \mathrm{~mW} / \mathrm{cm}^{2}$.

In addition to the depopulation beam intensity, transfer into the optical box is highly affected by both the duration and range over which the magnetic trap is translated. The optimal duration of this translation is mainly 


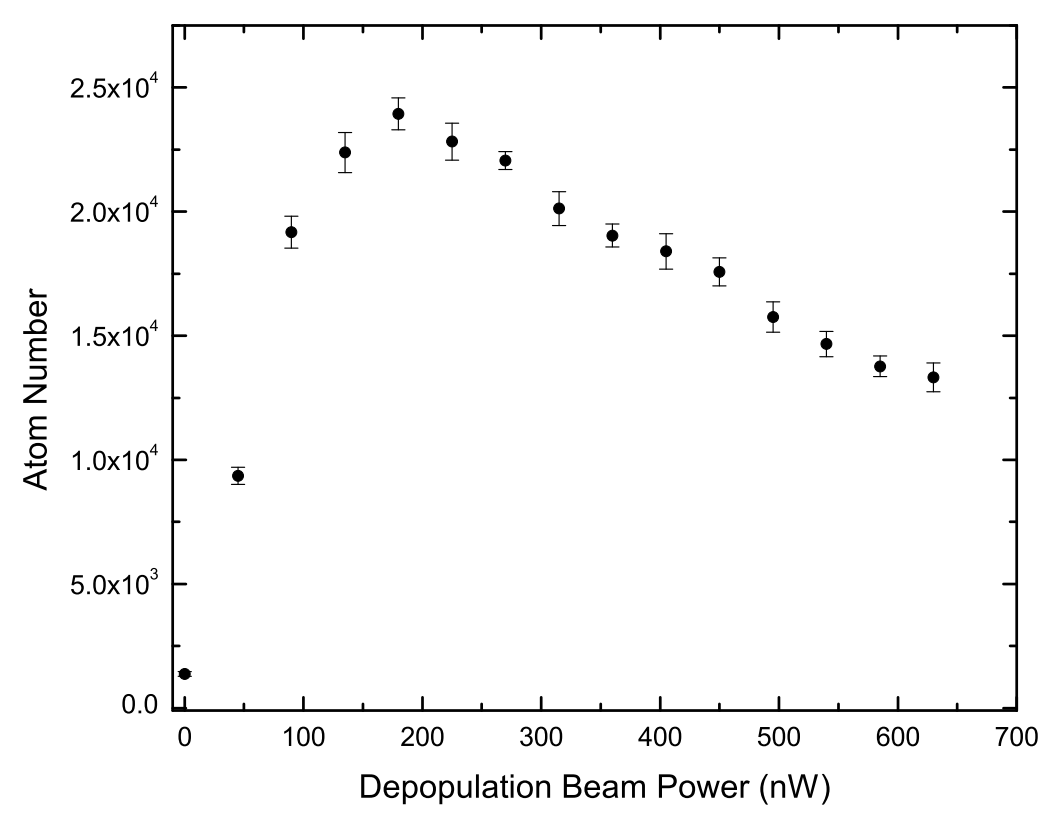

Figure 4.12: The number of atoms loaded into the optical box via single-photon atomic cooling as a function of the power in the depopulation beam.

dependent on two competing factors. Long translation times permit phasespace exploration by atoms in the magnetic trap, allowing a more complete exchange of kinetic for potential energy before an atom encounters the optical box. However, the finite lifetime of atoms in the optical box $(\tau=3.7 \pm 0.1 \mathrm{~s}$ in the presence of the depopulation beam) limits the translation time. We achieve highest density with a translation time of approximately $1.2 \mathrm{~s}$. Given this time scale, the translation range loading the largest atom number into the optical box is empirically determined. We translate the optical box from an initial separation (relative to the center of the magnetic trap) of $800 \mu \mathrm{m}$ to a final separation of $100 \mu \mathrm{m}$. 
To study the dynamics of the loading process, we look at the incremental loading for a constant translation velocity. We start with the center of the magnetic trap $800 \mu \mathrm{m}$ below the optical box and then translate it vertically at a velocity of $750 \mu \mathrm{m} / \mathrm{s}$. Figure 4.13 displays the fraction of atoms captured as a function of the final separation between the magnetic trap and the optical box. The slope of this plot indicates that the local loading rate increases with decreasing separation until about $100 \mu \mathrm{m}$. Additionally, it is clear from this plot that atom capture is not increased by translating beyond this point.

We study loading without translating the box (i.e. at a fixed separation) to understand the dynamics of single-photon atomic cooling in more detail. Figure 4.14 shows the number of atoms loaded into the optical box as a function of time for several separations. All curves exhibit a positive initial slope indicative of the local loading rate. As the magnetic trap is depleted by the depopulation beam, the loading rate decreases and the slope becomes dominated by trap losses. We find both the loading rate and the trap loss rate to be inversely related to the separation between the magnetic trap and optical box centers. The former reflects the dependence of the loading rate on the local density of magnetically trapped atoms. The latter suggests a higher rate of escape out of the optical box for smaller separations. This may be attributed in part to an increased temperature caused by collisions between atoms in the optical box and atoms in the magnetic trap. For the two smallest separations $(200 \mu \mathrm{m}, 400 \mu \mathrm{m})$ we calculate initial collision rates of $(0.8 \mathrm{~Hz}$, $0.5 \mathrm{~Hz})$ respectively. However, these rates diminish as the depopulation beam 


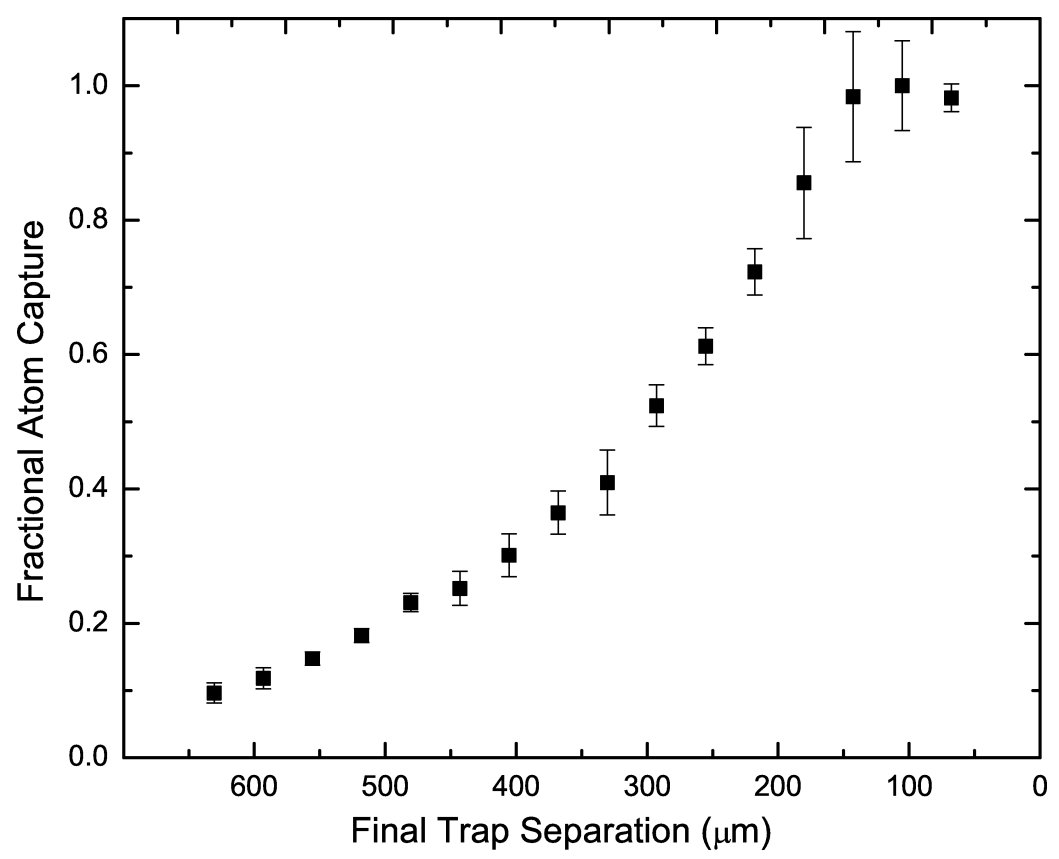

Figure 4.13: Incremental atom capture at a fixed translation velocity. The center of the magnetic trap is initially displaced $800 \mu \mathrm{m}$ below the optical box and is translated vertically at a velocity of $750 \mu \mathrm{m} / \mathrm{s}$. The endpoint of the translation is varied, and the atom capture, normalized to the maximum number, is plotted as a function of the final separation between the traps. Error bars indicate statistical uncertainties.

reduces the density of magnetically trapped atoms in the vicinity of the optical box. We thus consider collisions non-negligible for $\mathrm{t}<(250 \mathrm{~ms}, 500 \mathrm{~ms})$ which provides an upper bound of $(0.2,0.25)$ collisions per atom in the optical box. A large fraction of these collisions will cause immediate trap loss on account of the shallow box depth $(10 \mu \mathrm{K})$, but a few will raise the temperature. We believe, however, that this effect is overshadowed by atoms entering the optical box far from their classical turning points. In contrast to adiabatically 
translating the magnetically trapped atoms toward the optical box (as in Fig. 4.13), which yields a kinetic energy distribution independent of translation endpoint, we abruptly turn on the optical box and depopulation beam for the data in Fig. 4.14. In this situation, many atoms now transit the optical box far from their classical turning points, and if captured they contribute to an increased kinetic energy distribution and rate of escape.

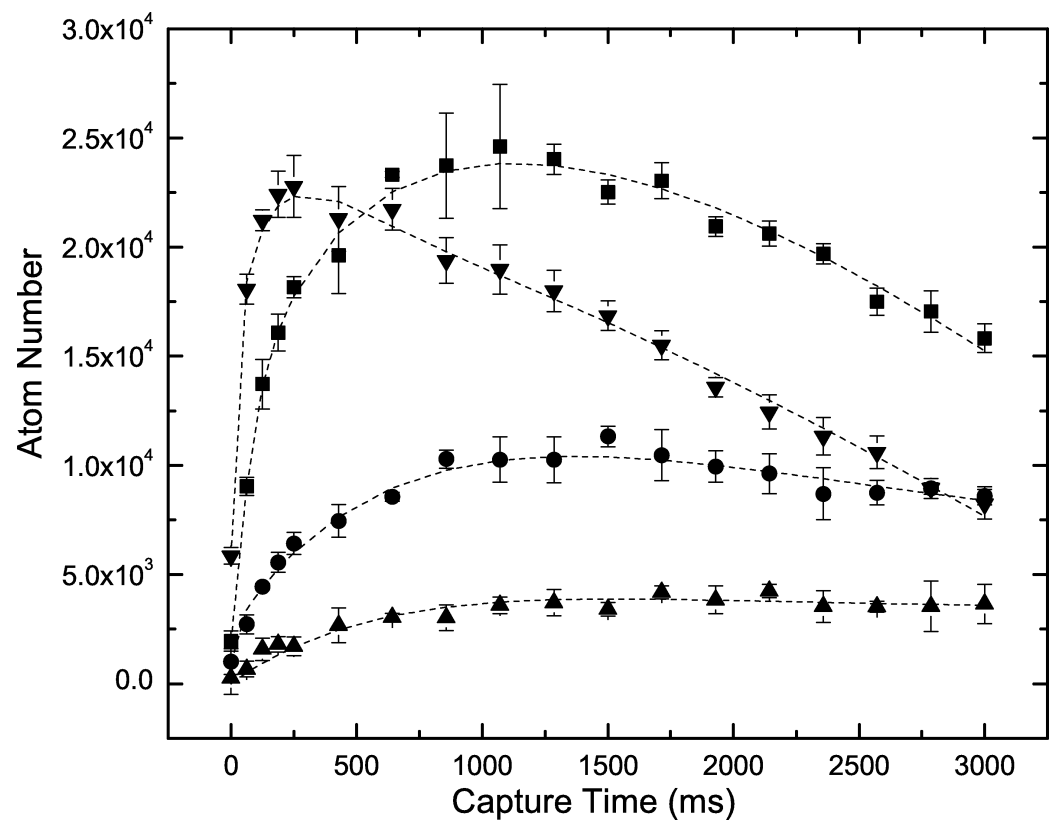

Figure 4.14: Captured atom number as a function of loading time. Data are given for separations between the optical and magnetic trap centers of $800 \mu \mathrm{m}$, $(\boldsymbol{\Delta}), 600 \mu \mathrm{m}(\mathbf{O}), 400 \mu \mathrm{m}(\mathbf{\square})$, and $200 \mu \mathrm{m}(\boldsymbol{\nabla})$. Error bars indicate statistical uncertainty, and dashed curves are drawn through the data points to guide the eye. The slopes are initially dominated by the loading rate into the optical box. After some time, the loading rate decreases due to the depletion of the magnetic trap, and the slopes become dominated by escape out of the box.

We performed Monte-Carlo simulations of the dynamics in the mag- 
netic trap and transfer into the optical box. Atom trajectories are propagated through phase-space, in which a subspace representing trapped states in the optical box has been defined. As atoms reach this subspace they are counted as trapped. These simulations show an inverse relationship between the loading rate and the separation between the magnetic trap and optical box centers in agreement with the experimental results.

Of utmost importance to the utility of this cooling technique is its ability to compress phase-space. With the single-photon atomic cooling scheme described in this section, we extract $1.5 \times 10^{5}$ atoms at a temperature of $7 \mu \mathrm{K}$ from the magnetic trap. We compare this with the number of atoms captured out of the magnetic trap without the depopulation beam. This is just a conservative dipole trap: atoms that are caught inside the box at low enough kinetic energy will be trapped, while all others will be lost. We measure a factor of $23 \pm 3$ increase in atom number using the single-photon atomic cooling method with nearly identical velocity distributions. We do not resolve the internal magnetic states in our measurement. The atoms in the magnetic trap are in the $F=2$ manifold, but can be in the $m_{F}=1$ and $m_{F}=2$ magnetic sublevels. The atoms caught in the optical box are in the $F=1$ manifold but can be in the $m_{F}=1$ and $m_{F}=0$ magnetic sublevels. The factor of 23 refers to atom number, not directly to phase space density. The increase in the latter would be a factor of 12 in the worst case scenario, if all the atoms in the magnetic trap were in the $F=2, m_{F}=2$ state and the atoms in the dipole trap were equally distributed between the two magnetic 
sublevels.

The increase in phase-space density demonstrated here is limited by technical constraints and does not represent a fundamental limit to this process. In the next section an improved version of the experiment is presented in which the atomic transfer efficiency is limited only by the dynamics of atoms in the magnetic trap.

\section{4 "Optical Trough" Configuration}

This section discusses the third, and current, experimental iteration of the single-photon cooling process [96]. As will be discussed in this section, the transfer efficiency of this iteration of the experiment is limited only by the dynamics of the atoms in the magnetic trap. In other words, all atoms which reach the depopulation beam with an energy less than the optical trap depth are cooled and transfered into the optical trap via the single-photon cooling process. The major changes made in this iteration were in the construction and placement of the optical dipole trap and the method of introducing magnetically trapped atoms into the depopulation beam near their classical turning points. Both of these improvements are discussed in more detail below. Together these changes resulted in a system performance increase of a factor of 15 .

As in the previous two iterations, atoms were initially loaded into a MOT, cooled with optical molasses, optically pumped, and then transfered into the magnetic trap. While we were able to vary both the number $N_{B}$ and 
temperature $T_{B}$ of atoms loaded into the magnetic trap, typical values used during these experiments were $N_{B} \approx 5 \times 10^{7}$ atoms and $T_{B} \approx 40 \mu \mathrm{K}$. The number and temperature of atoms loaded into the magnetic trap was varied by controlling the MOT beam detuning.

The presence of the repump beam during the MOT, optical molasses, and optical pumping stages ensures that the magnetically trapped atoms are in the $5^{2} S_{1 / 2}(F=2)$ hyperfine manifold. This manifold contains two magnetically trappable states: $\left|F=2, m_{F}=2\right\rangle$ and $\left|F=2, m_{F}=1\right\rangle$. The purpose of the optical pumping stage is to populate the $\left|F=2, m_{F}=2\right\rangle$ state preferentially over the $\left|F=2, m_{F}=1\right\rangle$ state. While helpful, this process is not $100 \%$ efficient and so there is a distribution of the two states in the magnetic trap. Later in this section we will calculate the amount of phase space compression achieved by the single-photon cooling process. To do this, we must find the phase space density of the atoms in the magnetic trap. This calculation requires knowledge of the number of magnetically trapped atoms in the $\left|F=2, m_{F}=2\right\rangle$ state. Unfortunately, our imaging method does not distinguish between atoms in the $\left|F=2, m_{F}=2\right\rangle$ and $\left|F=2, m_{F}=1\right\rangle$ states, so the atoms in these two states must be separated before imaging to get the number of interest. We achieved this separation by setting the magnetic field gradient to a value capable of levitating atoms in the $\left|F=2, m_{F}=2\right\rangle$ state against gravity, but not atoms in the $\left|F=2, m_{F}=1\right\rangle$ state. Figure 4.15 shows the number of atoms remaining in the magnetic trap as a function of the current in the quadrupole coils. As seen in this figure, below $\approx 8 \mathrm{~A}$ a sud- 
den decrease in atom number occurs. This is the current value below which

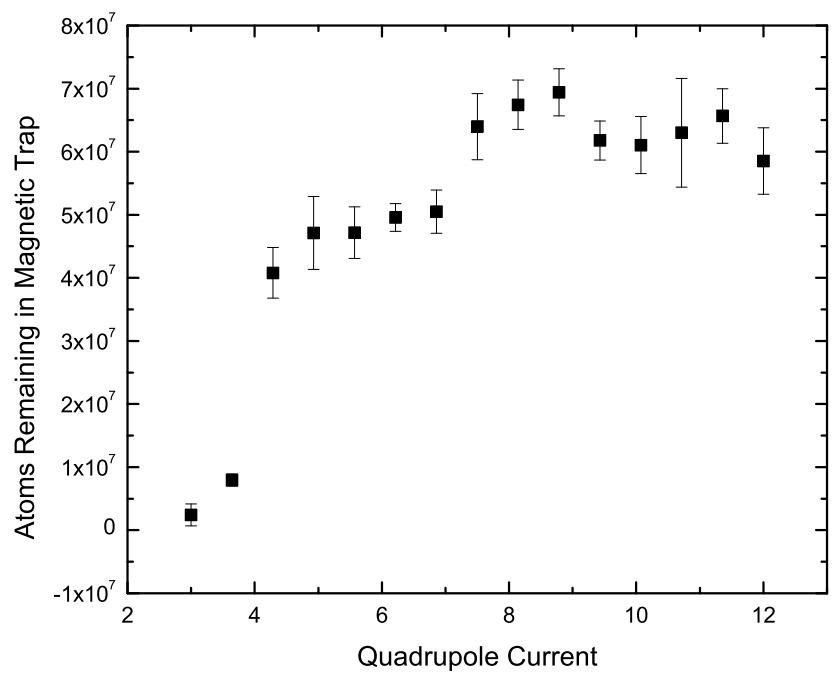

Figure 4.15: Number of atoms in magnetic trap as a function of the current in the quadrupole coils. Below a current of $\approx 8 \mathrm{~A}$ the field gradient is insufficient to support atoms in the $\left|F=2, m_{F}=1\right\rangle$ state against gravity.

atoms in the $\left|F=2, m_{F}=1\right\rangle$ are not supported against gravity. Likewise currents below $\approx 4 \mathrm{~A}$ do not support atoms in either state against gravity. Using this data we determined that approximately $70 \%$ of the magnetically trapped atoms are in $\left|F=2, m_{F}=2\right\rangle$ state and the remaining fraction are in the $\left|F=2, m_{F}=1\right\rangle$ state.

The optical dipole trap evolved in this iteration of the experiment from an "optical box" into an "optical trough." At the time we were using the optical box, we discovered that a box lid was not needed to confine atoms in all of the magnetic sublevels, gravity was sufficient to do the job for some. By removing the lid, we were able to divert more optical power from the laser 
used to construct it into the remaining five sheets, deepening the trap. The optical trough extends this line of reasoning by only requiring the use of four Gaussian sheets. Fig. 4.16 illustrates the geometry of the optical trough. Two

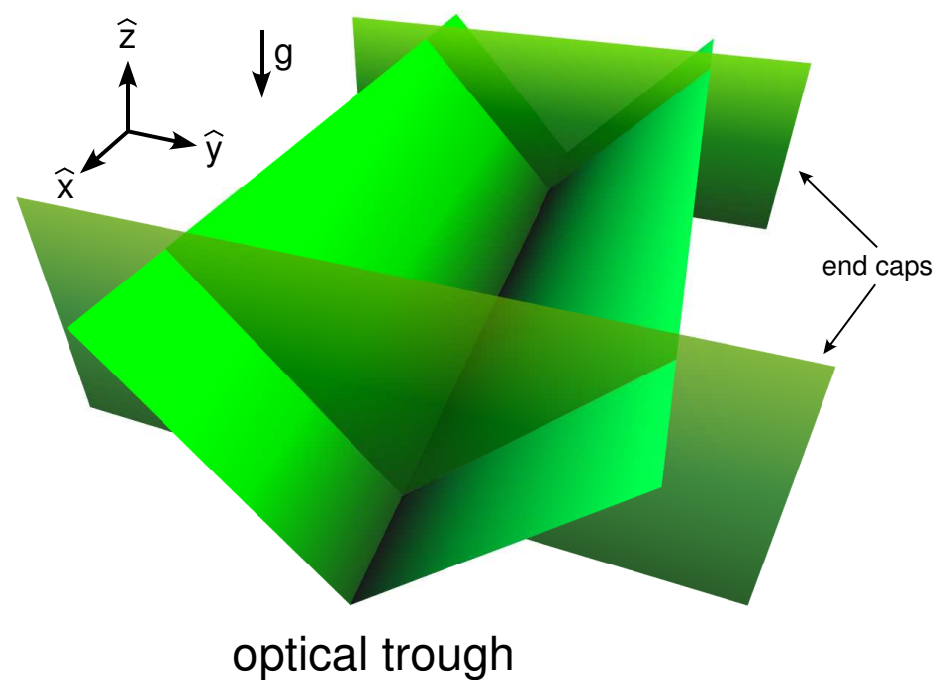

Figure 4.16: Geometry of the optical trough. Two Gaussian laser sheets in a ' $\mathrm{V}$ ' shape propagate along the $\hat{x}$ direction. Two more vertically elongated sheets propagating along the $\hat{y}$ axis intersect the ' $\mathrm{V}$ ' and serve as end caps. The end caps are separated by $110 \mu \mathrm{m}$. With gravity along the $\hat{z}$-axis, this trough creates a trapping potential in all directions.

Gaussian laser sheets in a 'V' shape propagate along the $\hat{x}$ direction. Two more vertically elongated sheets propagating along the $\hat{x}$ axis intersect the ' $\mathrm{V}$ ' and serve as end caps. The end caps are separated by $110 \mu \mathrm{m}$. With gravity along the z-axis, this trough creates a trapping potential in all directions. As before, each sheet is formed by asymmetrically focusing a circular beam with a cylindrical lens (see Fig. 3.21). At the location of the trap the sheets have $1 / e^{2}$ 
waists of $200 \times 10 \mu \mathrm{m}$. The measured and calculated trap depth is $\sim 10 \mu \mathrm{K}$.

Figure 4.17 shows the calculated potential of this optical dipole trap in the $\hat{y}-\hat{z}$ plane centered between the end caps along $\hat{x}$. This potential was calculated using Eq. 2.53, the trap geometry, and assuming each sheet had

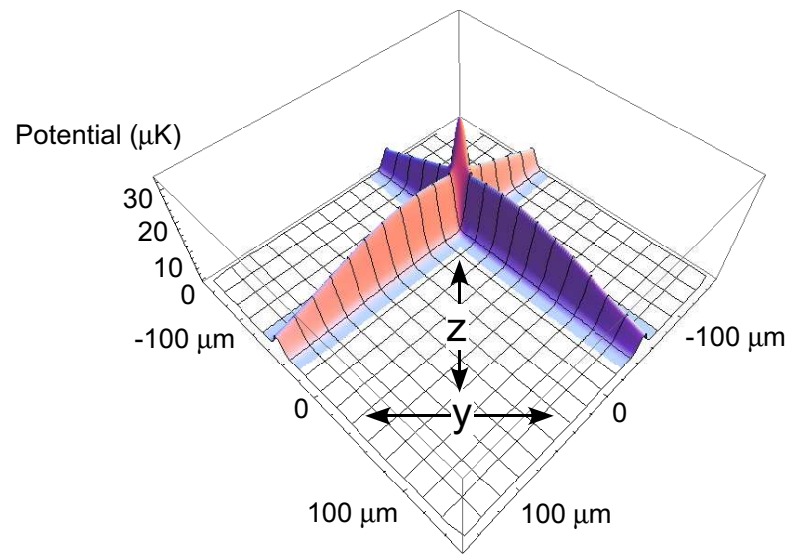

Figure 4.17: "Optical Trough" potential in the $\hat{y}-\hat{z}$ plane centered between the end caps.

$1 \mathrm{~W}$ of optical power. The presentation in this figure requires explanation. In this figure gravity $(-\hat{z})$ points from the lower corner of the plot towards the vertex of the potential. The trough levitates atoms against gravity and confines them along the $\hat{y}$ dimension with the aid of gravity. Figure 4.18 show the same potential from a side perspective allowing one to see the potential depth more easily. Figure 4.19(a) shows the calculated potential along a line parallel to $\hat{z}$ intersecting the trough at its geometric center. Figure 4.19(b) shows the calculated potential along the trough vertex. The curvature of the base of this potential is due to the finite Rayleigh length of the beams forming 


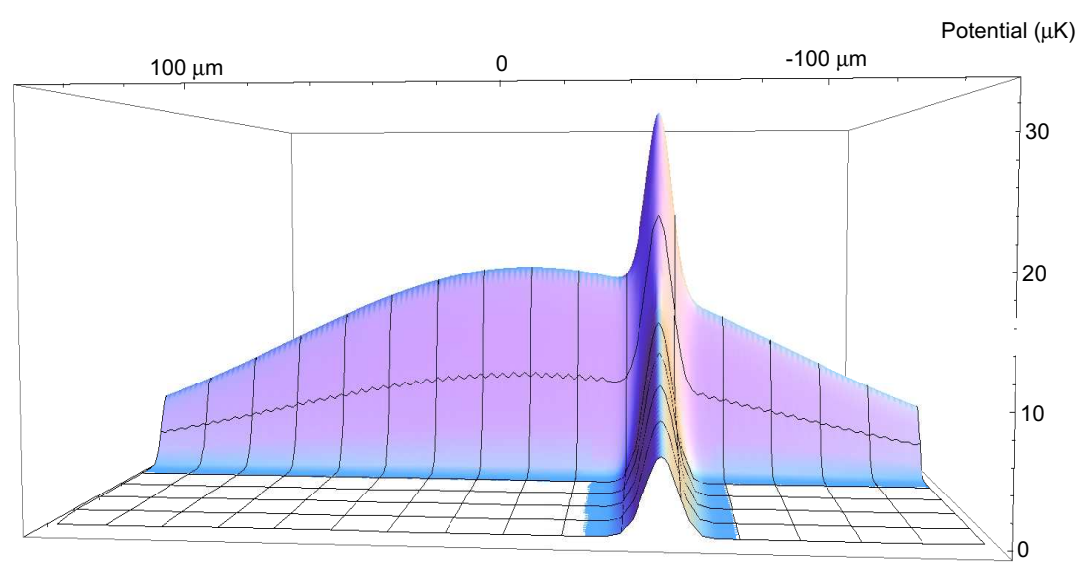

Figure 4.18: Side view of the optical trough potential displaying the trap depth.

the ' $\mathrm{V}$ '.

One further change made during this iteration was that we placed the optical trough below the magnetically trapped atoms in contrast to the last section in which the optical box was placed above the magnetic trap. Placing the trough below the magnetically trapped atoms is a more favorable geometry because atoms do not have to climb an optical potential hill to enter the optical trap. This is clearly advantageous for this cooling process because atoms climbing a potential hill subsequently fall down it, increasing their kinetic energy in the process. This places a lower limit on the final temperature of the sample.

As described in the previous two sections, the depopulation beam drives atoms from the $\left|F=2, m_{F}=2\right\rangle$ state into the $5^{2} S_{1 / 2}(F=1)$ manifold. It does this by exciting them into the $\left|F^{\prime}=1, m_{F}^{\prime}=1\right\rangle$ state from where they 

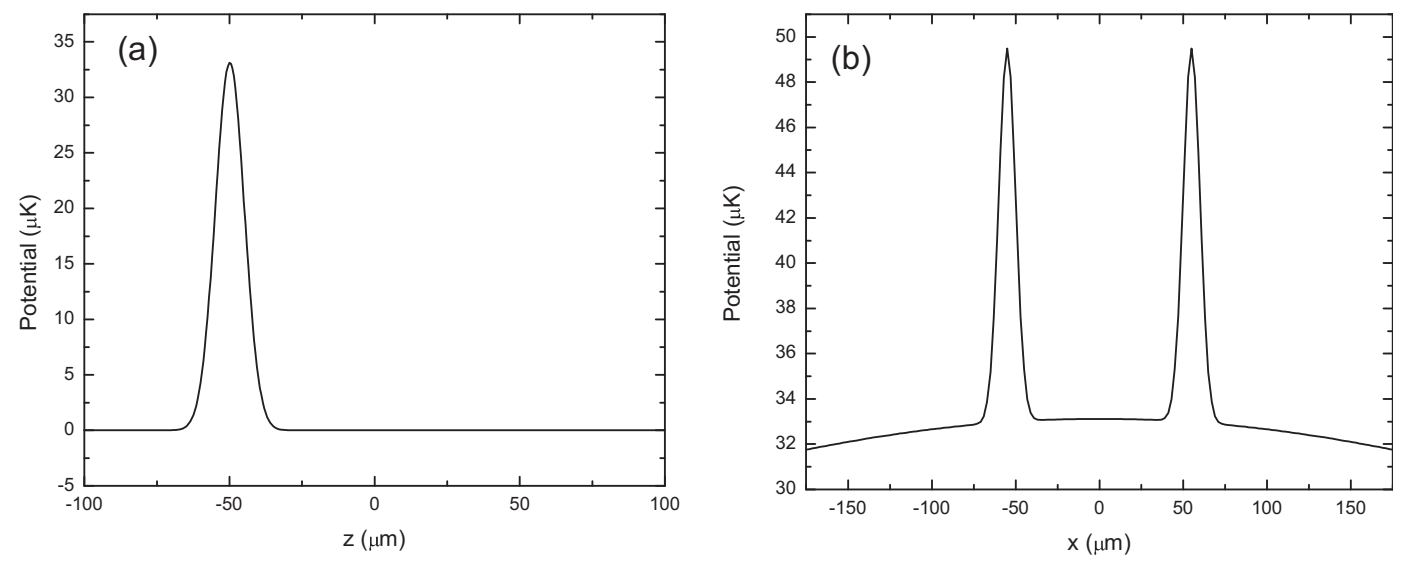

Figure 4.19: (a) Calculated potential along a line parallel to $\hat{z}$ intersecting the trough at its geometric center. (b) Calculated potential along the trough vertex.

spontaneously decay. Atom decay from this excited state into the $5^{2} S_{1 / 2}(F=$ 1) manifold with $84 \%$ probability (see Sec. 2.6). The remaining atoms decay back into the $5^{2} P_{3 / 2}(F=2)$ manifold and are subsequently re-excited by the depopulation beam. Because all atoms in the $5^{2} S_{1 / 2}(F=1)$ manifold couple to the magnetic field more weakly than atoms in the initial $\left|F=2, m_{F}=2\right\rangle$ state, they could in principle all be trapped. However, the branching ratio causes the final population to be predominantly in the $m_{F}=0,1$ sublevels. For the purpose of calculating the final phase-space density, we only consider atoms in the $m_{F}=0$ sublevel.

The depopulation beam travels parallel to the two Gaussian sheets which form a ' $\mathrm{V}$ ' as shown in Fig. 4.20. It is located a distance $h_{p}$ above the trough vertex and focused to a $1 / e^{2}$ waist of approximately $10 \mu \mathrm{m}$. This beam is detuned $35 \mathrm{MHz}$ below the $5^{2} S_{1 / 2}(F=2) \rightarrow 5^{2} P_{3 / 2}\left(F^{\prime}=1\right)$ transition. 


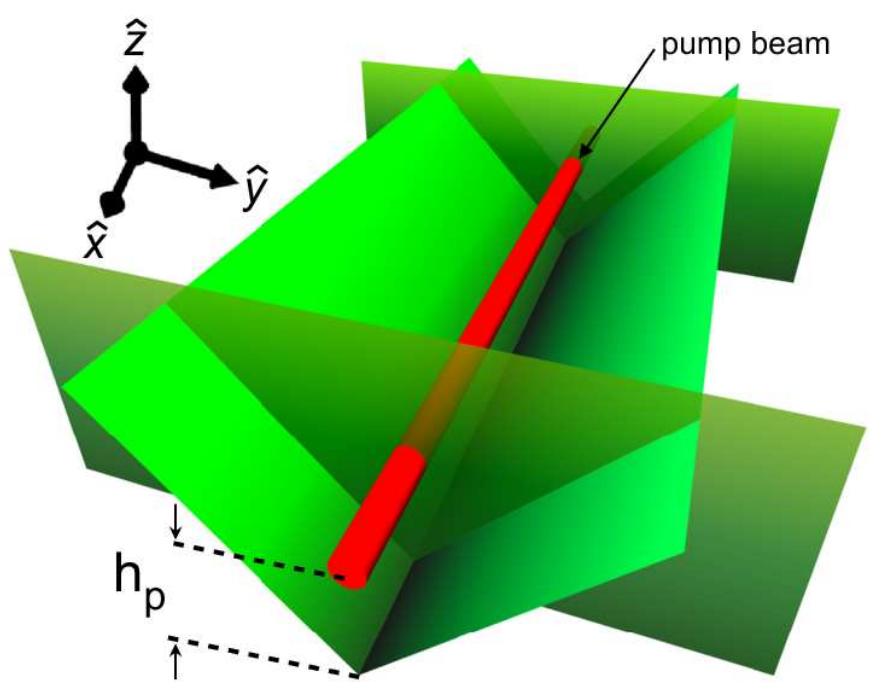

Figure 4.20: Geometry of the optical trough and depopulation beam. The depopulation beam is parallel to the $\hat{x}$ axis and is positioned a distance $h_{p}$ above the vertex of the trough.

The reason we choose to detune this beam from resonance can be explained by examining the data in Fig. 4.21. This plot shows the fluorescence signal from atoms transfered via single-photon atomic cooling as a function of the depopulation beam detuning from resonance with the $5^{2} S_{1 / 2}(F=2) \rightarrow$ $5^{2} P_{3 / 2}\left(F^{\prime}=1\right)$ transition frequency for a variety of intensities. The intensities used in this plot span nearly four orders of magnitude and were varied by placing neutral density filters in the beam path. As seen in the graph, for a given detuning an optimal intensity can be found. We believe this can be explained as follows. Below the optimal intensity, the depopulation beam does not excite atoms with unit probability and thus the transfer rate suffers. 


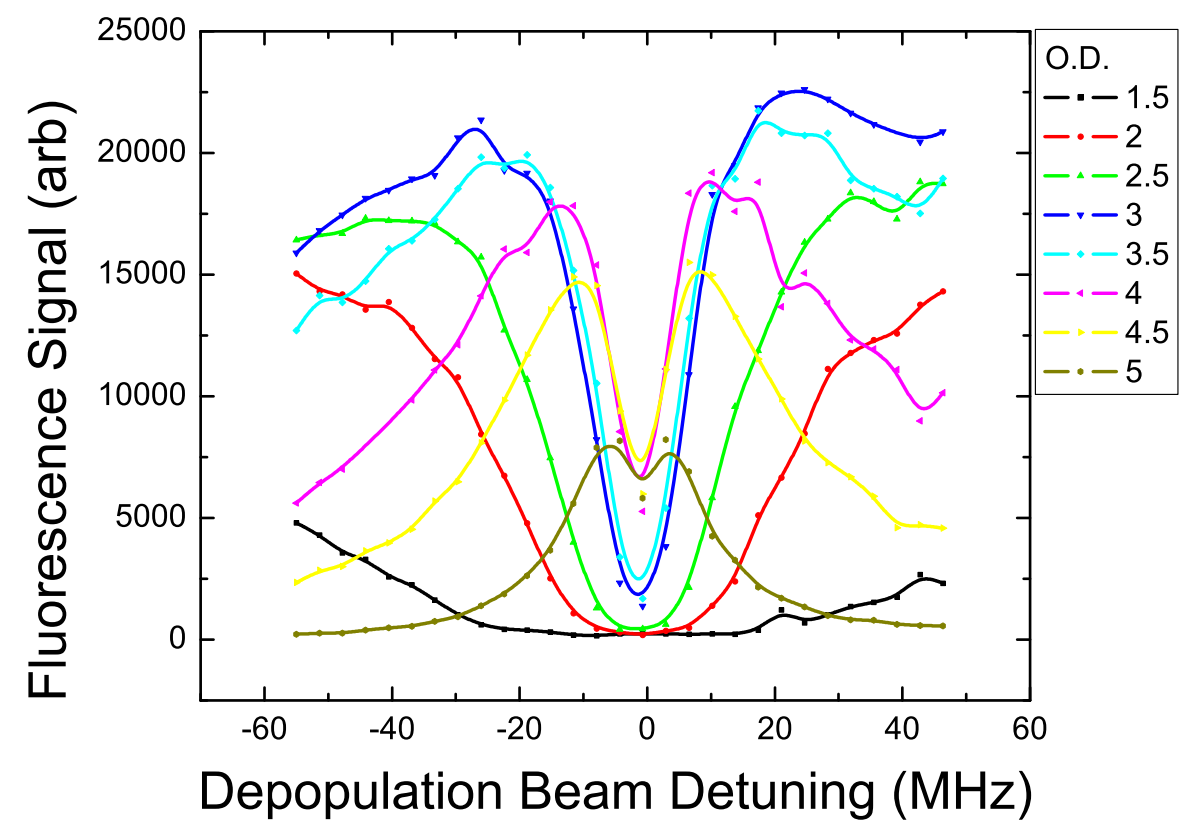

Figure 4.21: Fluorescence signal from atoms transfered via single-photon atomic cooling as a function of the depopulation beam detuning from resonance with the $5^{2} S_{1 / 2}(F=2) \rightarrow 5^{2} P_{3 / 2}\left(F^{\prime}=1\right)$ transition frequency for a variety of intensities. The intensity of the depopulation beam was attenuated with neutral density filters over nearly 4 orders of magnitude.

To understand why too high an intensity hurts the transfer efficiency we note that the depopulation beam scatters off of the chamber walls, bathing the magnetically trapped atoms in (near) resonant light. Of course, light of this frequency causes state transitions which cause atoms to be lost from the magnetic trap. This effect is beneficial to the single-photon cooling process when it occurs at the location of the depopulation beam focus. However, when the transitions occur away from the depopulation beam focus, due to scattered and reflected light, the result is magnetic trap loss. This removes atoms that 
could potentially have undergone the single-photon cooling process thereby reducing the transfer efficiency. Therefore, the intensity and detuning must be set strategically to maximize the efficiency of the transfer process. One may wonder why higher transfer efficiencies occur at larger frequency detunings. We believe that this can be understood by comparing the scattering rate in the depopulation beam to the rate in the magnetic trap due to scattered light. The rate at which atoms scatter near resonant light is found from Eq. 2.73 by multiplying $\rho_{22}$ by the excited state decay rate. The result is

$$
S_{\text {rate }}=\frac{s_{0} \Gamma / 2}{1+s_{0}+4(\Delta / \Gamma)^{2}},
$$

where $s_{0} \equiv I / I_{\text {sat }}$ is the saturation parameter, $\Gamma$ is the excited state decay rate, and $\Delta$ is the detuning from the resonance transition frequency. This equation shows that the scattering rate is not a linear function of beam intensity, rather the scattering rate begins to saturate when atoms spend a significant fraction of their time in the excited state. With this formula in mind, we are in a position to compare the scattering rate at the depopulation beam focus (high saturation parameter) with the rate in the magnetic trap (low saturation parameter) as a function of depopulation beam detuning from resonance. We begin by writing the scattering rate in the depopulation beam as

$$
S_{\text {depop }}=\frac{s_{0} \Gamma / 2}{1+s_{0}+4(\Delta / \Gamma)^{2}}
$$

While the amount of light scattered into the magnetic trap is unknown, it must be proportional to the intensity of the depopulation beam. Since $s_{0} \equiv I / I_{\text {sat }}$ 
we may write

$$
S_{m a g}=\frac{\alpha s_{0} \Gamma / 2}{1+\alpha s_{0}+4(\Delta / \Gamma)^{2}} .
$$

as an approximation to the scattering rate in the magnetic trap, where $\alpha$ represents the fraction of light scattered from the depopulation beam into the magnetic trap. Figure 4.22 is a plot of the ratio of the scattering rate at the depopulation beam focus to that in the magnetic trap. To make this plot I assumed $s_{0}=3$, a reasonable value. For lack of better terms I have

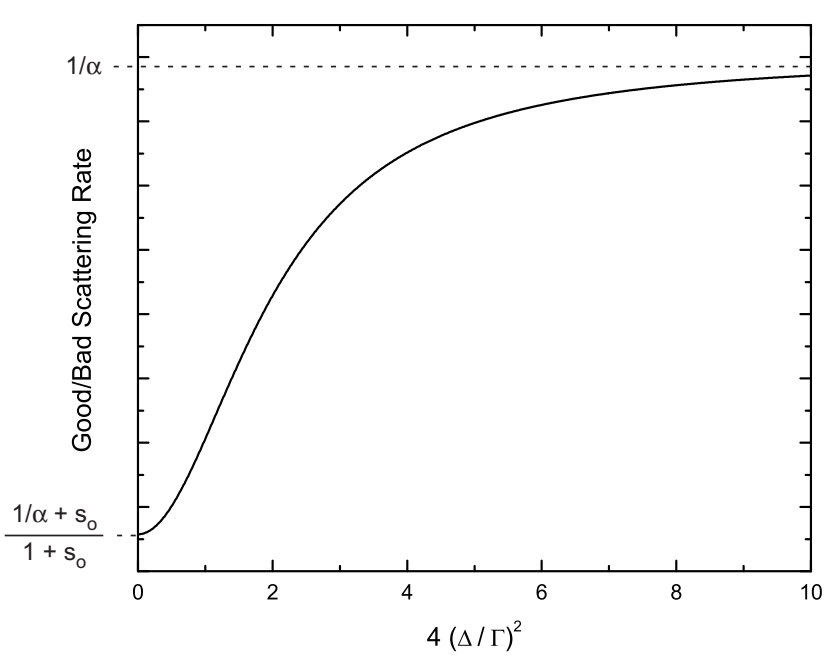

Figure 4.22: Ratio of the scattering rate in the depopulation beam (good) to the rate in the magnetic trap (bad) as a function of depopulation beam frequency detuning. For a given saturation parameter in the depopulation beam (here $s_{0}=3$ ) this ratio increases with increasing detuning.

labeled those scattering events that occur near the depopulation beam focus as "good," while those which happen in the magnetic trap as "bad." What this plot shows is that for a given saturation parameter in the depopulation beam, fewer atoms are scattered out of the magnetic trap at larger frequency 
detunings. The result of scattering events in the magnetic trap is, of course, trap loss. Trap loss reduces the atoms available to undergo the single-photon cooling process resulting in decreased transfer efficiency. By detuning the depopulation beam, we minimize this detrimental effect.

Before discussing the cooling process itself, I would like to show one more calculation for completeness. Figure 4.23 shows the combined potential due to the magnetic trap, optical trough, and gravity for atoms in the initial

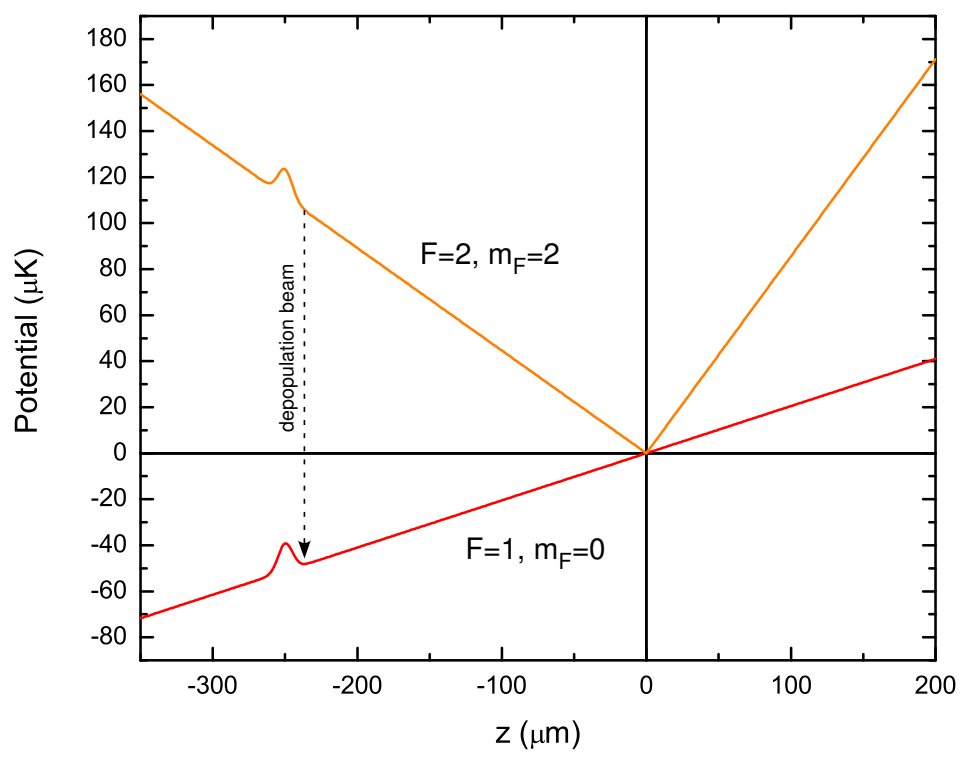

Figure 4.23: The combined potential due to the magnetic trap, optical trough, and gravity for atoms in the initial $\left|F=2, m_{F}=2\right\rangle$ and final $\left|F=1, m_{F}=0\right\rangle$ states along the vertical direction $(\hat{z})$. The dashed line indicates the preferred location of the depopulation beam to minimize residual kinetic energy.

$\left|F=2, m_{F}=2\right\rangle$ and final $\left|F=1, m_{F}=0\right\rangle$ states along the vertical direction $(\hat{z})$. The dashed line indicates the preferred location of the depopulation beam 
to minimize residual kinetic energy. Notice that in contrast to the potential produced by the optical box (Fig. 4.11), atoms do not have to climb a potential hill to reach the depopulation beam, resulting in a final population with less residual kinetic energy.

In this iteration of the experiment, the cooling process was initiated by adiabatically lowering the magnetic trapping potential. This is done by linearly ramping down the current in the quadrupole coils in a time $t_{r a m p}$, which is on the order of $1 \mathrm{~s}$. As the magnetic field gradient is reduced, the atomic cloud expands and the turning point of each atom (in the vertical direction) approaches the depopulation beam which is held a fixed distance below the magnetic trap. To ensure that each atoms encounters the depopulation beam near its classical turning point, the adiabaticity condition $\left\langle\tau_{B}\right\rangle / t_{\text {ramp }} \ll 1$ must be satisfied, where $\left\langle\tau_{B}\right\rangle$ is the average oscillation period in the magnetic trap. We found this scheme to be advantageous over simply moving the centers of the traps together, as in the previous two sections, because it reduced optical trap loss due to atomic collisions with magnetically trapped atoms.

As discussed above, when the atoms encounter the depopulation beam they are driven from the initial $\left|F=2, m_{F}=2\right\rangle$ state into the $5^{2} S_{1 / 2}(F=1)$ manifold. Once decoupled from the magnetic trap they fall into the optical trough where they are captured.

Once the cooling process is complete, atoms transfered into the optical trough via single-photon atomic cooling are imaged using the vertical probe beam (see Sec. 3.4.1). To remove any residual atoms, all magnetic fields 
are switched off causing non-optically trapped atoms to free fall under the influence of gravity, as in the last section. Then a beam resonant with the $5^{2} S_{1 / 2}(F=2) \rightarrow 5^{2} P_{3 / 2}(F=3)$ transition is used to blow away atoms not in the $5^{2} S_{1 / 2}(F=1)$ manifold. The remaining atoms have undergone the singlephoton cooling process. Then, the optical dipole beams are turned off and a probe beam propagating along the $\hat{z}$-axis illuminates the atoms for $200 \mu \mathrm{s}$. This beam is subsequently imaged onto a CCD camera and compared to a reference image taken with no atoms present. The result is integrated to yield the total number of atoms present in the sample (see Sec. 2.8.1). Temperature measurements obtained through the time-of-flight method are possible by varying the delay between the release of the cloud and illumination by the probe beam. Figure 4.24 shows an absorption image of approximately $1.5 \times 10^{5}$ atoms trapped in the optical trough. In this image false color is used to represent atomic density, magenta being the most dense. The rapid density fall-off along the $\hat{x}$ direction is due to the end caps. The density gradient along the $\hat{y}$ direction is due to the geometry of the trough.

To properly judge the performance of the single-photon cooling process, several effects introduced by the geometry of the optical trough should be considered. For example, the height of the pump beam above the trough vertex $h_{p}$ must be strategically set to optimize cooling. Figure 4.25 shows the effect of $h_{p}$ on both the number of atoms transfered into the optical trough via singlephoton cooling $N_{O}$ and the vertical temperature of the optically trapped atoms $T_{O}^{(z)}$. The temperature data was taken before atoms in the trough had time to 


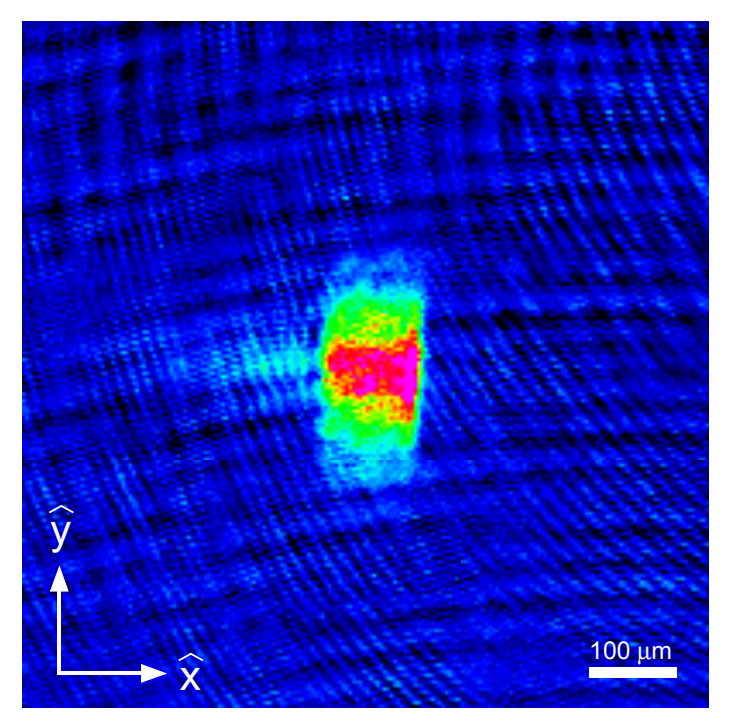

Figure 4.24: Absorption image of approximately $1.5 \times 10^{5}$ atoms trapped in the optical trough. In this image false color is used to represent atomic density, magenta being the most dense.

come to thermal equilibrium and therefore does not represent temperature in the thermodynamics sense, rather $T_{O}^{(z)}$ reflects the velocity distribution in the vertical direction (see Sec. 1.6 for a discussion of this use of "temperature"). $T_{O}^{(z)}$ increases monotonically with $h_{p}$, reflecting the energy gained by atoms during free fall from the depopulation beam to the trough vertex. Atoms which decay into the anti-trapped $\left|F=1, m_{F}=1\right\rangle$ state gain additional energy from the magnetic field gradient. For values of $h_{p}>100 \mu \mathrm{m}$, this increase in energy is sufficient to cause trap loss; atoms have enough energy to push through the bottom of the optical trough. To minimize the temperature of the transfered sample one should therefore minimize $h_{p}$ so that atoms are depopulated near the trough vertex. However, for small values of $h_{p}$ the optical dipole beams 


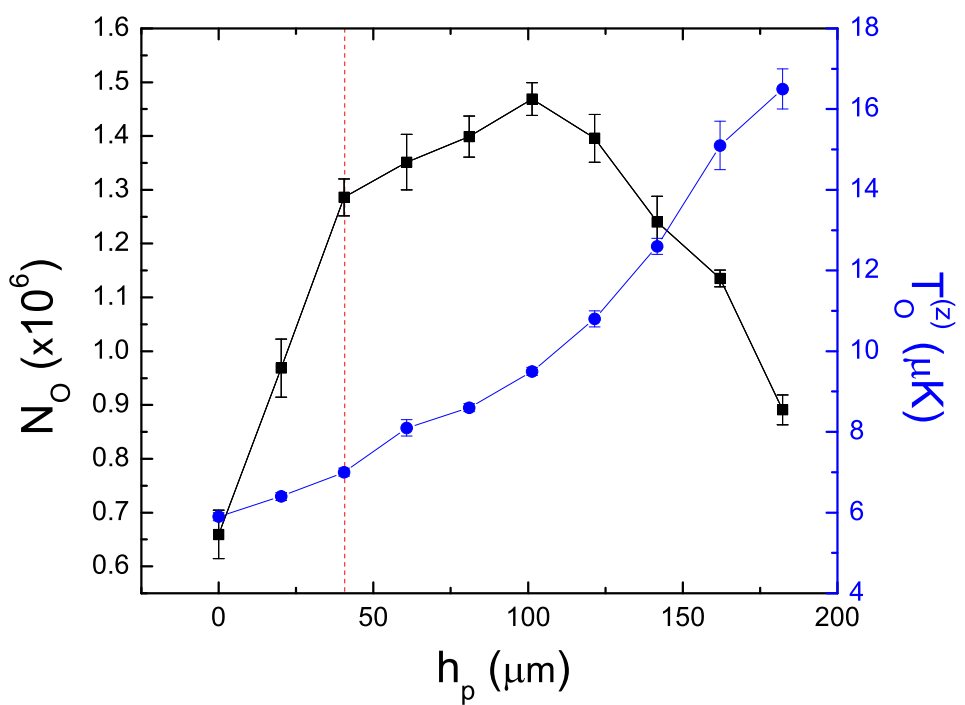

Figure 4.25: Number $(\mathbf{\square})$ and temperature $(\mathbf{O})$ of cooled atoms as a function of $h_{p}$ (height of the depopulation beam above the trough vertex). The positive slope of $T_{O}^{(z)}$ reflects energy gained by atoms in free fall. For $h_{p}>100 \mu \mathrm{m}$, the additional energy increases the loss rate from the optical trough. For $h_{p}<100 \mu \mathrm{m}$ spatial overlap of the pump beam and optical trough beams reduces the excitation probability and hence the capture rate. The highest phase-space density is achieved at $h_{p}=41 \mu \mathrm{m}$.

overlap the depopulation beam. This partial occlusion of the depopulation beam decreases the chance an atom will undergo the depopulating transition, resulting in a decreased transfer rate.

As discussed in Sec. 1.4 the recoil temperature $T_{r}=362 \mathrm{nK}$ is the fundamental limit to the single-photon cooling process. However, as indicated in Fig. 4.25, the final temperatures achieved during the single-photon cooling process are well above this limit even for the small values of $h_{p}$. If we where cooling a 1-D ensemble then this residual energy could only be attributed 
to capturing atoms away from their classical turning points (see Eq. 1.3 and related discussion). But this is not the case here because we are well into the adiabatic regime: $\left\langle\tau_{B}\right\rangle / t_{\text {ramp }} \approx 5 \times 10^{-} 3 \ll 1$. Indeed, we estimate the energy due to capturing atoms away from there classical turning points to be only $\approx 0.5 \mu \mathrm{K}$. This excess energy can be explained by noting that this process only cools the magnetically trapped atoms along the vertical dimension. Atoms transfered into the optical trap retain their horizontal velocity, and due to the geometry of the optical trough, energy in the $y$ dimension is quickly mixed with the $z$ dimension, accounting for the non-vanishing $T_{O}^{(z)}$.

The measure of the effectiveness of the single-photon cooling process is the amount of phase space compression it produces. For a given initial phase-space density, the compression is maximized when the final phase-space density is maximized. Here we define the phase-space density of our atomic ensembles in the usual manner [23]

$$
\rho=n \lambda_{d}^{3}=n\left[\frac{h}{\left(2 \pi M k_{B} T\right)^{1 / 2}}\right]^{3} \propto n T^{-3 / 2},
$$

where $n$ is the atom number density and $\lambda_{d}$ is the thermal de Broglie wavelength. The point on Fig. 4.25 corresponding to the highest phase-space density is located at $h_{p}=41 \mu \mathrm{m}$.

We now come to the issue of addressing transfer efficiency from the magnetic trap into the optical trough. To do this we compare the transfer efficiency of the single-photon cooling process to the maximum transfer efficiency expected in an adiabatic process transferring atoms between the two 
traps. If we model the ensembles in both the optical and magnetic trap with Maxwell-Boltzmann velocity distributions and Gaussian spatial distributions we can arrive at a simple analytical formula predicting the transfer efficiency between the two traps via an adiabatic process. Strictly speaking, the magnetic and optical potentials are not harmonic, and therefore the assumption of a Gaussian spatial distribution is clearly an approximation for our experiment. However, we maintain this approximation because of the simplicity and generality it affords our expression predicting the transfer efficiency. We estimate that this assumption leads to an error of roughly $15 \%$, which does not affect the conclusions drawn from comparing the model with experiment. Under an adiabatic transfer, the most atoms one could expect to transfer from the large-volume, deep magnetic trap into the small-volume, shallow optical trap is given by overlap of the the phase-space distribution of atoms in the two traps. Under the assumptions given above, we may write this overlap as

$$
\eta \equiv \frac{N_{O}}{N_{B}}=\prod_{i=\{x, y, z\}} \frac{\sigma_{O}^{(i)}}{\sigma_{B}^{(i)}} \sqrt{\frac{T_{O}^{(i)}}{T_{B}^{(i)}}},
$$

where $N_{O}\left(N_{B}\right), \sigma_{O}\left(\sigma_{B}\right)$, and $T_{O}\left(T_{B}\right)$ are the number, $1 / e$ radius and temperature of the atoms in the optical (magnetic) trap, respectively. In this formula the product runs over all three orthogonal dimensions to allow for trap anisotropy. Furthermore, this form is only valid when $\left(\sigma_{O}^{(i)}, T_{O}^{(i)}\right) \leq\left(\sigma_{B}^{(i)}, T_{B}^{(i)}\right)$ is true.

Now we must consider the effect of single-photon atomic cooling on the transfer process. In a non-interacting ensemble, the single-photon atomic 
cooling process compresses one dimension of the magnetic trap completely both in position and momentum space (neglecting a photon recoil). This can be expressed in Eq. 4.5 by setting the product over the vertical dimension $(\hat{z})$ to 1 . With this assumption we can write an upper bound on the expected transfer efficiency of the single-photon cooling process as

$$
\eta_{s p c}=\prod_{i=\{x, y\}} \frac{\sigma_{O}^{(i)}}{\sigma_{B}^{(i)}} \sqrt{\frac{T_{O}^{(i)}}{T_{B}^{(i)}}} \propto\left(\sigma_{B} \sqrt{T_{B}}\right)^{-2},
$$

where $T_{B}=T_{B}^{(i)}$ reflects a thermalized magnetic trap, and $\sigma_{B} \equiv \sigma_{B}^{(x)}=2 \sigma_{B}^{(y)}$ reflects the anisotropic geometry of our magnetic quadrupole trap. We see then, for a fixed optical trap geometry and depth that $\eta_{s p c}$ is determined by the initial conditions in the magnetic trap. This expression can be simplified even further by noting that for a thermalized ensemble the size of the cloud in the magnetic trap is function of its temperature $\sigma_{B}=\sigma_{B}\left(T_{B}\right)$. Figure 4.26 shows a plot of the size of the magnetic cloud as a function of magnetic trap temperature. We find that a linear fit of the measured radii in this regime yields $\sigma_{B}=\left(25.8+5.5 T_{B} \mu \mathrm{K}^{-1}\right) \mu \mathrm{m}$.

Figure 4.27 shows the experimentally measured transfer efficiencies along with the predicted upper bound, given in Eq. 4.6, for several magnetic trap temperatures. Data in this figure show fair agreement with Eq. 4.6 below $40 \mu \mathrm{K}$, but there is a trend of increasing efficiency, with respect to the upper bound, with increasing temperature. We believe that this can be understood by noting that our derivation of $\eta_{s p c}$ is for a non-interacting ensemble. In this case, the initial trajectories of atoms in the magnetic trap fully determine the 


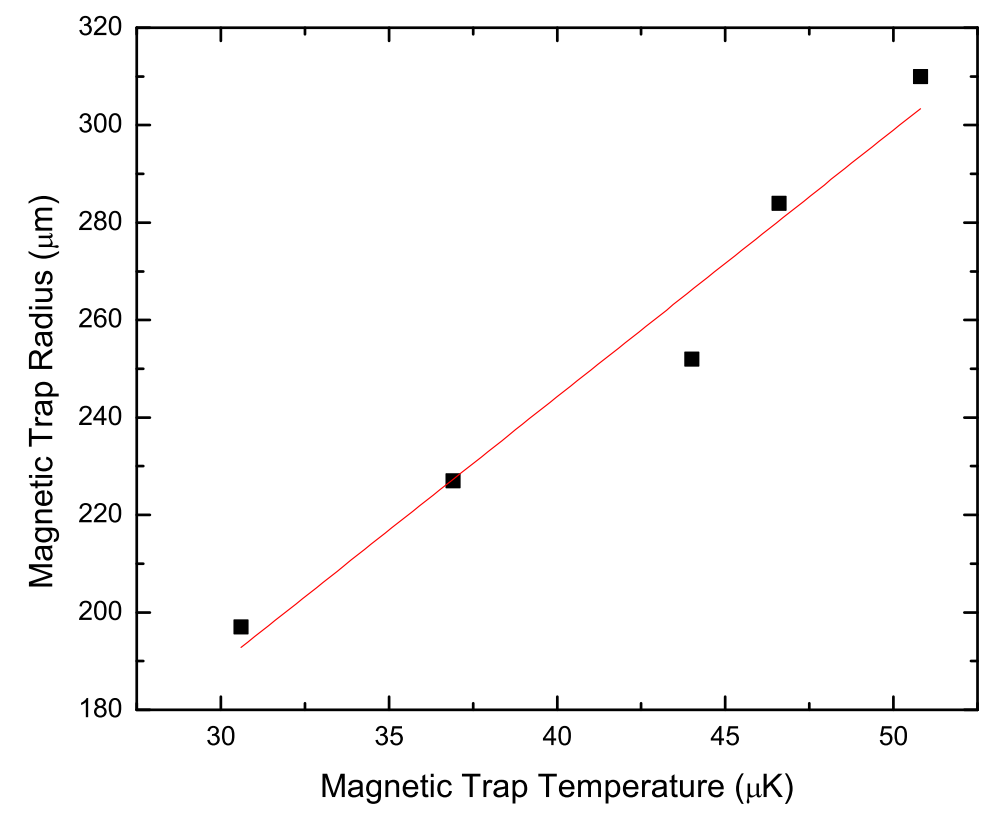

Figure 4.26: Radius of the atomic cloud in the magnetic trap as a function of temperature. We find that a linear fit of the measured radii in this regime yields $\sigma_{B}=\left(25.8+5.5 T_{B} \mu \mathrm{K}^{-1}\right) \mu \mathrm{m}$.

dynamics of the cooling process. Only a small fraction of those atoms, represented by Eq. 4.6, will become trapped in the optical trough. In reality, atoms in the magnetic trap weakly interact through collisions. The average single particle collision rate in the magnetic trap is given by

$$
\Gamma=N^{-1} \int n(\vec{r})^{2} \sigma_{s}\left\langle v_{r}\right\rangle d \vec{r}
$$

where $\mathrm{N}$ is the total number of trapped atoms, $n(\vec{r})$ is the atom number density, $\sigma_{s}$ is the s-wave scattering cross section, and $\left\langle v_{r}\right\rangle=\sqrt{16 k_{B} T / \pi m}$ is the mean relative speed in a three-dimensional Boltzmann distribution. The inset in Fig. 4.27 shows the calculated average single particle collision rate in the 


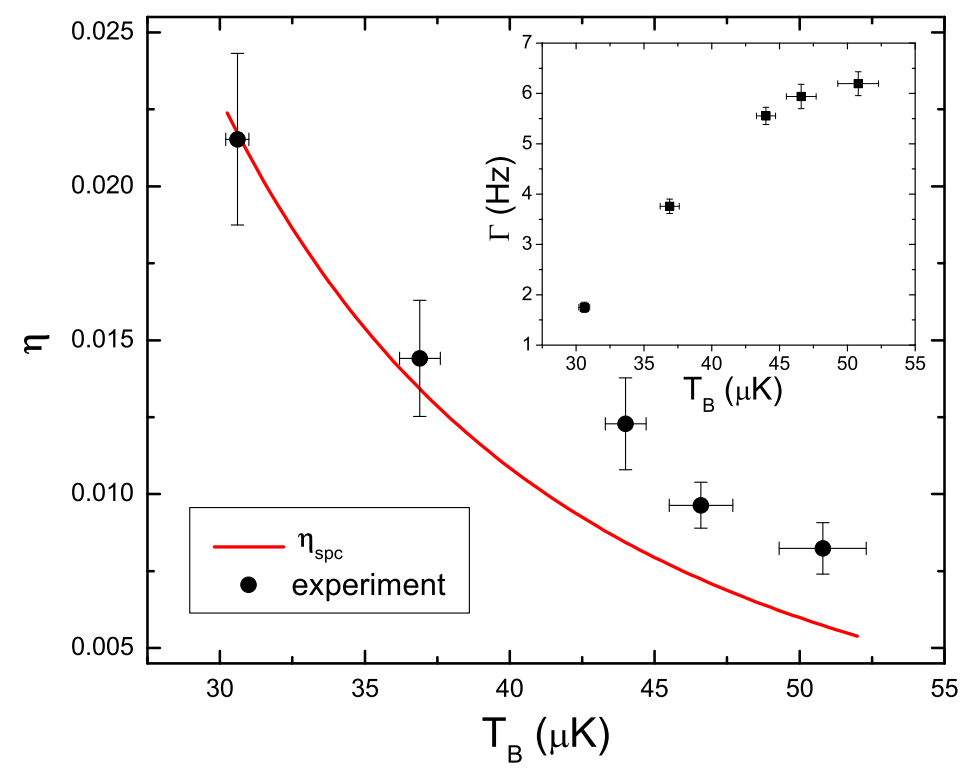

Figure 4.27: Experimentally measured transfer efficiencies (circles) as a function of magnetic trap temperature. The solid line represents the predicted upper bound capture efficiency given by Eq. 4.6. Inset shows the calculated average single particle collision rate in the magentic trap.

magnetic trap as a function of temperature. As shown in the figure, there is a monotonically increasing trend. The effect of collisions is to rethermalize the magnetic trap during the single-photon cooling process, repopulating trappable trajectories as they are depleted by the depopulation beam. Therefore, transfer efficiencies are expected to be enhanced for weakly interacting ensembles, in agreement with the measured trend.

To gain further insight into the transfer process, we compared the rate 
atoms were removed from the magnetic trap by the depopulation beam to the rate they were loaded into the optical trough. Figure 4.28 shows the fraction of atoms remaining in the magnetic trap as a function of the percent of the current ramp down completed. As seen in the figure, atoms are removed from the magnetic trap by the depopulation beam at a nearly constant rate

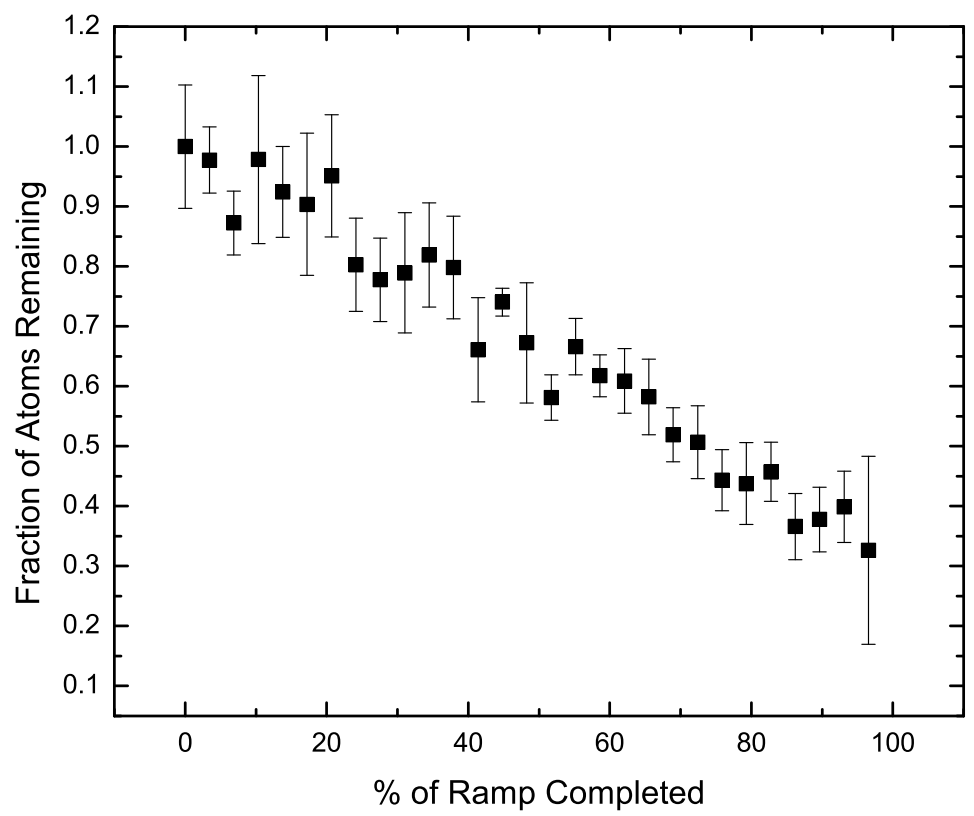

Figure 4.28: Fraction of atoms remaining in the magnetic trap as a function of the percentage of current ramp down completed. The total number of atoms depopulated from the magnetic trap in this graph was approximately $3 \times 10^{7}$ atoms.

during the cooling process. In this figure, the total number of atoms removed from the magnetic trap by the depopulation beam is roughly $3 \times 10^{7}$ atoms. For comparison, Fig. 4.29 shows the number of atoms accumulated in the optical trough as a function of the current in the quadrupole coils. Despite 
the constant rate at which atoms are depopulated from the magnetic trap, the

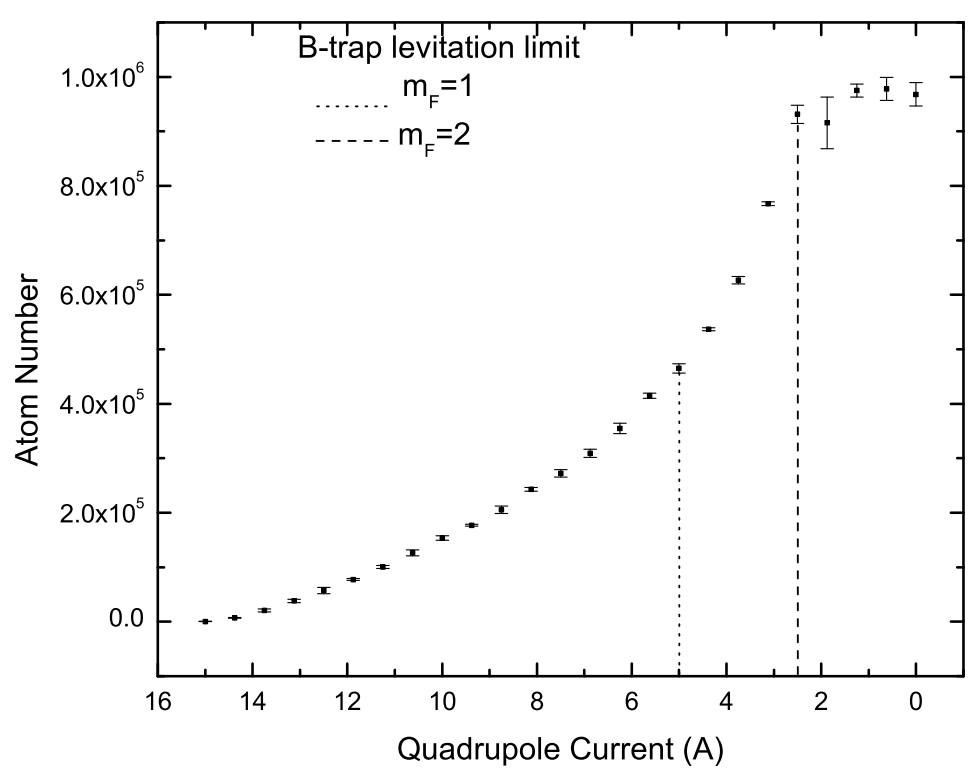

Figure 4.29: Atom accumulation in the optical trough as a function of the current in the quadrupole coils. Vertical lines indicate the point at which the magnetic field gradient produced by the current is no longer sufficient to levitate atoms against gravity for atoms in the $m_{F}=1$ and $m_{F}=2$ magnetic substates.

loading rate into the optical trough is clearly non-linear. This graph indicates that atoms are more efficiently loaded at the end of the cooling sequence, when the magnetic field gradient is low. This trend may be explained as follows. As the magnetic gradient is reduced, atoms in the magnetically anti-trapped $\left|F=1, m_{F}=1\right\rangle$ state gain less energy as they travel from the depopulation beam to the optical trough vertex or walls. Therefore a smaller fraction of these atoms will have sufficient energy to push through the bottom or sides of the trough's potential. Also, at lower magnetic field gradients the magnetically 
trappable $\left|F=1, m_{F}=-1\right\rangle$ state is not levitated out of the optical trough and becomes trappable in the optical trough. Together, these effects result in an increased transfer efficiency.

The maximum transfer efficiency we have measured is $2.2(3) \%$. However, it is clear from Eq. 4.6 that this number can be trivialy increased by increasing the phase space overlap of the two traps. This could be accomplished by reducing the size and temperature of the magnetic trap or increasing the size and depth of the optical trough. It should also be noted that one can use Eq. 4.6 to find an expression for the increase in the phase-space density of a non-interacting ensemble undergoing this process:

$$
\frac{\rho_{O}}{\rho_{B}}=\frac{\sigma_{B}^{(z)} \sqrt{T_{B}^{(z)}}}{\sigma_{O}^{(z)} \sqrt{T_{O}^{(z)}}} .
$$

For a fixed optical trough geometry and depth, this ratio increases with $T_{B}$ in spite of a corresponding decrease in transfer efficiency.

With initial magnetic trap parameters $T_{B}=53 \mu \mathrm{K}$ and $\sigma_{B}=515 \mu \mathrm{m}$, we have transfered $3.3 \times 10^{5}$ atoms at a temperature of $4.3 \mu \mathrm{K}$ with $0.3 \%$ transfer efficiency. This corresponds to a peak phase-space density of $4.9(3) \times$ $10^{-4}$, which is roughly a 350 -fold increase over the phase-space density of the magnetic trap.

\subsection{Future Directions}

The power of the single-photon cooling technique will only be fully demonstrated when it has been successfully applied to a species not amenable 
to other existing cooling techniques. The prospects of such a demonstration are particularly promising in light of recent work with supersonic beams which produced trapped samples of paramagnetic atoms [97,98] and molecules [99102] at tens of millikelvins in a simple room-temperature apparatus. The general nature of the single-photon cooling technique means that it can potentially be adapted to cool and trap a large portion of these species, many of which cannot be laser cooled with existing techniques. There has even been a proposal to use this technique to cool molecules [103], which existing laser cooling techniques have failed to cool due to their complicated energy level structures.

The main focus in our lab however will be the application of singlephoton atomic cooling to hydrogenic isotopes. The methods described in this dissertation, with appropriate modifications, are well suited to cooling and trapping all three isotopes. In short, the proposed technique begins by seeding a supersonic beam of neon with the hydrogenic isotope under study. After entrainment, the hydrogenic beam will be brought to rest and trapped magnetically using an "atomic coilgun." In fact, atomic hydrogen has already been trapped in this manner [98]. Once trapped, the single-photon cooling process will proceed in a manner similar to that discussed in this dissertation. The species will initially be in the $\left|F=1, m_{F}=1\right\rangle$ state. These atoms will be depopulated near their classical turning points by driving them into the $2 \mathrm{~s}$ manifold via a two-photon transition at $243 \mathrm{~nm}$. This long lived metastable state will be quenched by application of a DC electric field mixing it with 
the short lived $2 p$ state. Atoms which decay into the $F=0$ manifold will be trapped in an optical dipole trap formed from a standing wave of light inside a build-up cavity. This process is outlined in Fig. 4.30, which shows the relevant energy levels in hydrogen.

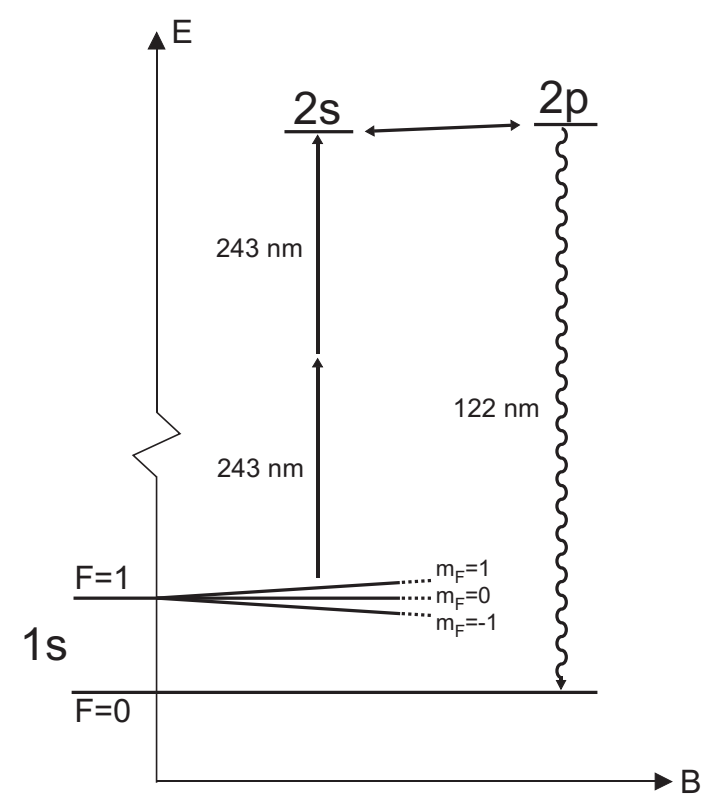

Figure 4.30: Energy structure of Hydrogen relavant to single-photon atomic cooling. Atoms magnetically trapped in the $\left|F=1, m_{F}=1\right\rangle$ state will be depopulated near their classical turning points by driving them into the $2 \mathrm{~s}$ manifold via a two photon transition at $243 \mathrm{~nm}$. This long lived metastable state will be quenched by application of a DC electric field mixing it with the short lived $2 p$ state. Atoms which decay into the $F=0$ manifold will be trapped in an optical dipole trap formed by a standing wave of light inside a build-up cavity.

The first goal after trapping and cooling hydrogen and its isotopes will be to push the limits of ultrahigh precision spectroscopy, especially needed for 
tritium. Additionally, cooled and trapped tritium may serve as an ideal system for determining the neutrino rest mass, as shown in a recent concept paper [104]. Perhaps most exciting to me is the proposed use of single-photon atomic cooling on anti-hydrogen $[105,106]$ once it has been magnetically trapped [107]. 


\section{Bibliography}

[1] R. Frisch, "Experimental demonstration of Einstein's radiation recoil," Z. Physik 86, 42 (1933).

[2] T. Hansch and A. Schawlow, "Cooling of gases by laser radiation," Opt. Commun. 13, 68 (1975).

[3] D. Wineland and H. Dehmelt, "Proposed $10^{14} \Delta v<v$ laser fluorescence spectroscopy on $\mathrm{T}^{+}$mono-ion oscillator III," Bull. Am. Phys. Soc. 20, 637 (1975).

[4] D. Wineland, R. Drullinger, and F. Walls, "Radiation-pressure cooling of bound resonant absorbers," Phys. Rev. Lett. 40, 1639 (1978).

[5] W. Neuhauser, M. Hohenstatt, P. Toschek, and H. Dehmelt, "Opticalsideband cooling of visible atom cloud confined in parabolic well," Phys. Rev. Lett. 41, 233 (1978).

[6] J. Prodan, A. Migdall, W.D. Phillips, I. So, H. Metcalf, and J. Dalibard, "Stopping atoms with laser light," Phys. Rev. Lett. 54, 992 (1985).

[7] W. Ertmer, R. Blatt, J. L. Hall, and M. Zhu, "Laser manipulation of atomic beam velocities: Demonstration of stopped atoms and velocity reversal," Phys. Rev. Lett. 54, 996 (1985). 
[8] S. Chu, L. Hollberg, J.E. Bjorkholm, A. Cable, and A. Ashkin, "Threedimensional viscous confinement and cooling of atoms by resonance radiation pressure," Phys. Rev. Lett. 55, 48 (1985).

[9] A. L. Migdall, J. V. Prodan, and W. D. Phillips, "First observation of magnetically trapped neutral atoms," Phys. Rev. Lett. 54, 2596 (1985).

[10] E. L. Raab, M. Prentiss, A. Cable, S. Chu, and D.E. Pritchard, "Trapping of neutral sodium atoms with radiation pressure," Phys. Rev. Lett. 59, 2631 (1987).

[11] P. Lett, R. N. Watts, C. Westbrook, W. D. Phillips, P. L. Gould, and H. J. Metcalf, "Observation of atoms laser cooled below the Doppler limit," Phys. Rev. Lett. 61, 169 (1988).

[12] J. Dalibard and C. Cohen-Tannoudji, "Laser cooling below the Doppler limit by polarization gradients: simple theoretical models," J. Opt. Soc. Am. B 6, 2023 (1989).

[13] K. B. Davis, M. O. Mewes, M. R. Andrews, N. J. van Druten, D. S. Durfee, D. M. Kurn, and W. Ketterle, "Bose-Einstein condensation in a gas of sodium atoms," Phys. Rev. Lett. 75, 3969 (1995).

[14] M. H. Anderson, J. R. Ensher, M. R. Matthews, C. E. Wieman, and E. A. Cornell, "Observation of Bose-Einstein condensation in a dilute atomic vapor," Science 269, 198 (1995). 
[15] C. C. Bradley, C. A. Sackett, J. J. Tollett, and R. G. Hulet, "Evidence of Bose-Einstein condensation in an atomic gas with attractive interactions," Phys. Rev. Lett. 75, 1687 (1995).

[16] G. Santarelli, P. Laurent, P. Lemonde, A. Clairon, A. G. Mann, S. Chang, A. N. Luiten, and C. Salomon, "Quantum projection noise in an atomic fountain: A high stability cesium frequency standard," Phys. Rev. Lett. 82, 4619 (1999).

[17] S. T. Cundiff, J. Ye, and J. L. Hall, "Optical frequency synthesis based on mode-locked lasers," Rev. Sci. Instrum. 72, 3749 (2001).

[18] T. W. Hansch, A. L. Schawlow, and G. W. Series, "The spectrum of atomic hydrogen," Sci. Am. 240, 72 (1979).

[19] N. J. Fisch, J. M. Rax, and I. Y. Dodin, "Current drive in a ponderomotive potential with sign reversal," Phys. Rev. Lett. 91, 205004 (2003).

[20] A. Ruschhaupt and J. G. Muga, "Atom diode: A laser device for a unidirectional transmission of ground-state atoms," Phys. Rev. A 70, 061604(R) (2004).

[21] A. Ruschhaupt, J. G. Muga, and M. G. Raizen, "Improvement by laser quenching of an atom diode: a one-way barrier for ultra-cold atoms," $J$. Phys. B: At. Mol. Opt. Phys. 39, L133 (2006). 
[22] M. G. Raizen, A. M. Dudarev, Qian Niu, and N. J. Fisch, "Compression of atomic phase space using an asymmetric one-way barrier," Phys. Rev. Lett. 94, 053003 (2005).

[23] C. J. Pethick and H. Smith, Bose-Einstein Condensation in Dilute Gases (Cambridge University Press, Cambridge, 2002).

[24] J. C. Maxwell, Theory of Heat (Longmans, Green and Co., London, 1875), 4th ed.

[25] D. V. Schroeder, An Introduction to Thermal Physics (Addison Wesley Longman, New York, 2000).

[26] L. Szilard, "On the decrease in entropy in a thermodynamic system by the intervention of intelligent beings," Z. Physik 53, 840 (1929).

[27] L. Brillouin, "Maxwell's demon cannot operate: Information and entropy. I," J. Appl. Phys. 22, 334 (1951).

[28] L. Brillouin, "Physical entropy and information. II," J. Appl. Phys. 22, $338(1951)$.

[29] L. Brillouin, Science and Information Theory (Academic, New York, 1956).

[30] C.E. Shannon, "A mathematical theory of communication." Bell System Tech. J. 27, 379 (1948). 
[31] E.T. Jaynes, "Information theory and statistical mechanics," Phys. Rev. 106, 620 (1957).

[32] C.H. Bennett, "Notes on the history of reversible computation," IBM J. Res. Dev. 32, 16 (1988).

[33] M.O. Scully, "Extracting work from a single thermal bath via quantum negentropy," Phys. Rev. Lett. 87, 220601 (2001).

[34] A. Ruschhaupt, J.G. Muga, and M.G. Raizen, "One-photon atomic cooling with an optical maxwell demon valve," J. Phys. B: At. Mol. Opt. Phys. 39, 3833 (2006).

[35] S. J. van Enk and G. Nienhuis, "Entropy production and kinetic effects of light," Phys. Rev. A 46, 1438 (1992).

[36] D. Mohl, G. Petrucci, L. Thorndahl, and S. van der Meer, "Physics and technique of stochastic cooling," Phys. Rep. 58, 73 (1980).

[37] S. van der Meer, "Stochastic cooling and the accumulation of antiprotons," Rev. Mod. Phys. 57, 689 (1985).

[38] H. J. Metcalf and P. van der Straten, Laser Cooling and Trapping (Springer; Corrected edition, New York, 1999).

[39] K. Huang, Statistical Mechanics (Wiley; Second edition, U.S.A., 1987).

[40] D. R. Lide (Ed.), CRC Handbook of Chemistry and Physics (CRC Press, Boca Raton, 2001), $82^{\text {nd }}$ ed. 
[41] M.P. Bradley, J.V. Porto, S. Rainville, J.K. Thompson, and D.E. Pritchard, "Penning trap measurements of the masses of ${ }^{133} \mathrm{Cs},{ }^{87,85} \mathrm{Rb}$, and ${ }^{23} \mathrm{Na}$ with uncertainties $\leq 0.2 \mathrm{ppb}, "$ Phys. Rev. Lett. 83, 4510 (1999).

[42] C.B. Alcock, V.P. Itkin, and M.K. Horrigan, "Vapor pressure equations of the metallic elements: 298-2500K," Canadian Metallurgical Quarterly 23, 309 (1984).

[43] Daniel A. Steck, "Rubidium 87 D Line Data," http://steck.us/alkalidata (revision 2.1, 1 September 2008).

[44] A. Corney, Atomic and Laser Spectroscopy (Oxford University Press, USA, 2006).

[45] E. Arimondo, M. Inguscio, and P. Violino, "Experimental determinations of the hyperfine structure in the alkali atoms," Rev. Mod. Phys. 49, 31 (1977).

[46] V. Gerginov, A. Derevianko, and C.E. Tanner, "Observation of the nuclear magnetic octupole moment of ${ }^{133} \mathrm{Cs}, "$ Phys. Rev. Lett. 91, 072501 (2003).

[47] L. Armstrong Jr., Theory of the Hyperfine Structure of Free Atoms (Wiley-Interscience, New York, 1971).

[48] S. Bize, Y. Sortais, M.S. Santos, C. Mandache, A. Cleiron, and C. Salomon, "High-accuracy measurement of the ${ }^{87} \mathrm{Rb}$ ground-state hyperfine splitting in an atomic fountain," Europhys. Lett. 45, 558 (1999). 
[49] J. Ye, S. Swartz, P. Jungner, and J.L. Hall, "Hyperfine structure and absolute frequency of the ${ }^{87} \mathrm{Rb} 5 P_{3 / 2}$ state," Optics Letters 21, 1280 (1996).

[50] D.J. Griffiths, Introduction to Quantum Mechanics (Prentice Hall, New Jersey, 1995).

[51] J.J. Sakurai, Modern Quantum Mechanics (Addison-Wesley, New York, 1994), revised ed.

[52] B. Odom, D. Hanneke, B. D’Urso, and G. Gabrielse, "New measurement of the electron magnetic moment using a one-electron quantum cyclotron," Phys. Rev. Lett. 97, 030801 (2006).

[53] L. Labzowsky, I. Goidenko, and P. Pyykko, "Estimates of the boundstate QED contributions to the g-factor of valence ns electrons in alkali metal atoms," Phys. Lett. A 258, 31 (1999).

[54] H.A Bethe and E.E. Salpeter, Quantum Mechanics of One- and Twoelectron Atoms (Springer-Verlag, Berlin, 1957).

[55] W. Wing, "On neutral particle trapping in quasistatic electromagnetic fields," Prog. Quant. Elect. 8, 181 (1984).

[56] T. Bergeman, G. Erez, and H. Metcalf, "Magnetostatic trapping fields for neutral atoms," Phys. Rev. A 35, 1535 (1987). 
[57] Spiegel and Liu, Mathematical Handbook of Formulas and Tables (McGrawHill, 1999), $2^{\text {nd }}$ ed.

[58] D. Budker, D. Kimball, and D. DeMille, Atomic Physics (Oxford University Press, New York, 2008).

[59] G.A. Askar'yan, "Effects of the gradient of a strong electromagnetic beam on electrons and atoms," Sov. Phys. JETP 15, 1088 (1962).

[60] A. Ashkin, "Acceleration and trapping of particles by radiation pressure," Phys. Rev. Lett. 24, 156 (1970).

[61] A. Ashkin, "Trapping of atoms by resonance radiation pressure," Phys. Rev. Lett. 40, 729 (1978).

[62] J. E. Bjorkholm, R. R. Freeman, A. Ashkin, and D. B. Pearson, "Observation of focusing of neutral atoms by the dipole forces of resonanceradiation pressure," Phys. Rev. Lett. 41, 1361 (1978).

[63] S. Chu, J.E. Bjorkholm, A. Ashkin, and A. Cable, "Experimental observation of optically trapped atoms," Phys. Rev. Lett. 57, 314 (1986).

[64] A.P. Kazantsev, "Acceleration of atoms by a resonance field," Sov. Phys. JETP 36, 861 (1973).

[65] R.J. Cook, "Atomic motion in resonant radiation: An application of Ehrenfest's theorem," Phys. Rev. A 20, 224 (1979). 
[66] J.P. Gordon and A. Ashkin, "Motion of atoms in a radiation trap," Phys. Rev. A 21, 1606 (1980).

[67] J.D. Jackson, Classical Electrodynamics (Wiley, New York, 1998), $3^{\text {rd }}$ ed.

[68] R. Grimm and M. Weidemuller, "Optical dipole traps for neutral atoms," arXiv:physics/9902072v1 (1999).

[69] R. Loudon, The Quantum Theory of Light (Oxford, 1962), $2^{\text {nd }}$ ed.

[70] C. J. Foot, Atomic Physics (Oxford University Press, New York, 2005).

[71] D. E. Pritchard, E. L. Raab, V. Bagnato, C. E. Wieman, and R. N. Watts, "Light traps using spontaneous forces," Phys. Rev. Lett. 57, 310 (1986).

[72] D.M. Brink and G.R. Satchler, Angular Momentum (Oxford University Press, USA, 1994), 3rd ed.

[73] A. R. Edmonds, Angular Momentum in Quantum Mechanics (Princeton University Press, 1996).

[74] A. Messiah, Quantum Mechanics (Wiley, New York, 1966).

[75] I. Sobelman, Atomic Spectra and Radiative Transitions (Springer-Verlag, Berlin, 1992).

[76] D. A. Varshalovich, A. N. Moskalev, and V. K. Khersonskii, Quantum Theory of Angular Momentum (World Scientific, Singapore, 1988). 
[77] B. R. Judd, Operator Techniques in Atomic Spectroscopy (Princeton University Press, New Jersey, 1988).

[78] M. Rotenberg, N. Metropolis, R. Birins, and J. Wooten Jr., The $3 j$ and 6j Symbols (Technology Press, Cambridge, 1959).

[79] W. Demtroder, Laser Spectroscopy (Springer; Second edition, New York, 1996).

[80] D. S. Weiss, E. Riis, Y. Shevy, P. J. Ungar, and S. Chu, "Optical molasses and multilevel atoms: experiment," J. Opt. Soc. Am. B 6, 2072 (1989).

[81] W. Ketterle, D.S. Durfee, and D.M. Stamper-Kurn, "Making, probing and understanding Bose-Einstein condensates," arXiv:cond-mat/9904034v2 (1999).

[82] C.-S. Chuu, F. Schreck, T. P. Meyrath, J. L. Hanssen, G. N. Price, and M. G. Raizen, "Direct observation of sub-poissonian number statistics in a degenerate Bose gas," Phys. Rev. Lett. 95, 260403 (2005).

[83] J.L. Hanssen, Controlling Atomic Motion: From Single Particle Classical Mechanics to Many Body Quantum Dynamics, Ph.D. thesis, The University of Texas at Austin (2004).

[84] T.P. Meyrath, Experiments with Bose-Einstein Condensation in an Optical Box, Ph.D. thesis, The University of Texas at Austin (2005). 
[85] C.-S. Chuu, Direct Study of Quantum Statistics in a Degenerate Bose Gas, Ph.D. thesis, The University of Texas at Austin (2006).

[86] W. Wohlleben, F. Chevy, K. Madison, and J. Dalibard, "An atom faucet," Eur. Phys. J. D 15, 237 (2001).

[87] J.H. Moore, C.C. Davis, and M.A. Coplan, Building Scientific Apparatus (Westview Press, Boulder, CO, 2003), $3^{\text {rd }}$ ed.

[88] B.G. Klappauf, Experimental Studies of Quantum Chaos with Trapped Cesium, Ph.D. thesis, The University of Texas at Austin (1998).

[89] D.A. Steck, Quantum Chaos, Transport, and Decoherence in Atom Optics, Ph.D. thesis, The University of Texas at Austin (2001).

[90] W.H. Oskay, Atom Optics Experiments in Quantum Chaos, Ph.D. thesis, The University of Texas at Austin (2001).

[91] C. E. Wieman and L. Hollberg, "Using diode lasers for atomic physics," Rev. Sci. Instrum. 62, 1 (1991).

[92] A. Steinbach K. B. MacAdam and C.Wieman, "A narrow-band tunable diode laser system with grating feedback, and a saturated absorption spectrometer for Cs and Rb," Am. J. Phys. 60, 1098 (1992).

[93] A.E. Siegman, Lasers (University Science Books, Sausalito, CA, 1986).

[94] G. N. Price, S. T. Bannerman, E. Narevicius, and M. G. Raizen, "Singlephoton atomic cooling," Laser Phys. 17, 1 (2007). 
[95] G. N. Price, S. T. Bannerman, K. Viering, E. Narevicius, and M. G. Raizen, "Single-photon atomic cooling," Phys. Rev. Lett. 100, 093004 (2008).

[96] S. T. Bannerman, G. N. Price, K. Viering, and M. G. Raizen, "Singlephoton cooling at the limit of trap dynamics: Maxwell's demon near maximum efficiency," New J. Phys. 11, 063044 (2009).

[97] E. Narevicius, A. Libson, C. G. Parthey, I. Chavez, J. Narevicius, U. Even, and M.G. Raizen, "Stopping supersonic beams with a series of pulsed electromagnetic coils: An atomic coilgun," Phys. Rev. Lett. 100 , 093003 (2008).

[98] S. D. Hogan, A. W. Wiederkehr, H. Schmutz, and F. Merkt, "Magnetic trapping of hydrogen after multistage zeeman deceleration," Phys. Rev. A 101, 143001 (2008).

[99] E. Narevicius, A. Libson, C. G. Parthey, I. Chavez, J. Narevicius, U. Even, and M.G. Raizen, "Stopping supersonic oxygen with a series of pulsed electromagnetic coils: A molecular coilgun," Phys. Rev. A 77, 051401(R) (2008).

[100] H. L. Bethlem, G. Berden, and G. Meijer, "Decelerating neutral dipolar molecules," Phys. Rev. Lett. 83, 1558 (1999).

[101] S. Y. T. van de Meerakker, P. H. M. Smeets, N. Vanhaecke, R. T. Jongma, and G. Meijer, "Deceleration and electrostatic trapping of $\mathrm{OH}$ 
radicals," Phys. Rev. Lett. 94, 023004 (2005).

[102] B. C. Sawyer, B. L. Lev, E. R. Hudson, B. K. Stuhl, M. Lara, J. L. Bohn, and J. Ye, "Magnetoelectrostatic trapping of ground state $\mathrm{OH}$ molecules," Phys. Rev. Lett. 98, 253002 (2007).

[103] E. Narevicius, S. T. Bannerman, and M. G. Raizen, "Single-photon molecular cooling," New J. Phys. 11, 055046 (2009).

[104] M. Jerkins, J. Majors, J. R. Klein, and M. G. Raizen, "Using cold atoms to measure neutrino mass," arXiv:0901.3111v3 [nucl-ex] (2009).

[105] M. Amoretii et al., "Production and detection of cold antihydrogen atoms," Nature 419, 456 (2002).

[106] G. Gabrielse et al., "Background-free observation of cold antihydrogen with field-ionization analysis of its states," Phys. Rev. Lett. 89, 213401 (2002).

[107] M. G. Raizen, "Comprehensive control of atomic motion," Science 324, 1403 (2009). 


\section{Vita}

Gabriel Noam Price was born in Denver, Colorado on November 20, 1978 as the son of Neal and Anne Price. He graduated from South High School in Denver, Colorado in 1997. In 2003 he received a Bachelor of Arts degree in Physics with highest honors from The University of Colorado at Boulder. Gabriel joined Mark G. Raizen's research group at The University of Texas at Austin in August of 2003.

Permanent address: 1060 Cottonwood Cir

Golden, CO 80401

This dissertation was typeset with $\mathrm{I}_{\mathrm{E}} \mathrm{X}^{\dagger}$ by the author.

${ }^{\dagger} \mathrm{LAT} \mathrm{EX}$ is a document preparation system developed by Leslie Lamport as a special version of Donald Knuth's TEX Program. 